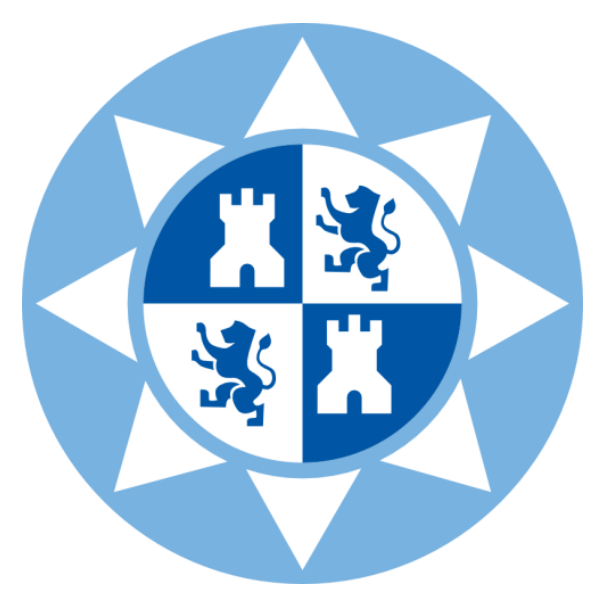

Universidad Politécnica de Cartagena

Departamento de Tecnologías de la Información y las Comunicaciones

\title{
Measurement Techniques Enhancements for MIMO 4G Mobile Communication Systems. Extension of Mode Stirred Reverberation Chambers (MSRCs) Emulation Capabilities
}

Juan Diego Sánchez Heredia 



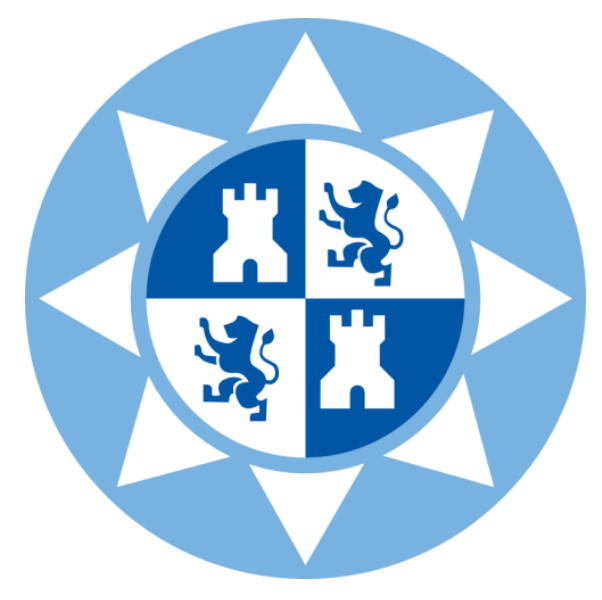

Universidad Politécnica de Cartagena

Departamento de Tecnologías de la Información y las Comunicaciones

\section{Measurement Techniques Enhancements for MIMO 4G Mobile Communication Systems. Extension of Mode Stirred Reverberation Chambers (MSRCs) Emulation Capabilities}

Juan Diego Sánchez Heredia

Director

Antonio Manuel Martínez González 



\section{Universidad}

Politécnica

de Cartagena

DT-12

\section{CONFORMIDAD DE SOLICITUD DEAUTORIZACIÓN DE DEPÓSITO DE} TESIS DOCTORAL POR EL/LA DIRECTOR/A DE LA TESIS

D./Da. Antonio Manuel Martínez González Director/a de la Tesis doctoral "Measurement

Techniques Enhancements for MIMO 4 G Mobile Communication Systems. Extension of

Mode Stirred Reverberation Chambers (MSRCs) Emulation Capabilities"

\section{INFORMA:}

Que la referida Tesis Doctoral, ha sido realizada por D/D ${ }^{\mathrm{a}}$. Juan Diego Sánchez Heredia, dando mi conformidad para que sea presentada ante la Comisión de Doctorado.

La rama de conocimiento por la que esta tesis ha sido desarrollada es:

$\square \quad$ Ciencias

- Ciencias Sociales y Jurídicas

X Ingeniería y Arquitectura

En Cartagena, a 4 de jumio de 2012

LOS/LAS DIRECTORES/AS DE LA TESIS

Fdo.: Antonıo M. Martinez González 



\section{CONFORMIDAD DE DEPOSITO DE TESIS DOCTORAL POR LA COMISIÓN ACADÉMICA DEL PROGRAMA}

D/D ${ }^{a}$. Francisco Alhama López, Presidente/a de la Comisión Académica del Programa de doctorado de Tecnologías Industriales,

\section{INFORMA:}

Que la Tesis Doctoral titulada; "Measurement Techniques Enhancements for MIMO $4 G$ Mobile Communication Systems. Extension of Mode Stirred Reverberation Chambers (MSRCs) Emulation Capabilities", ha sido realizada por D. Juan Diego Sánchez Heredia, bajo la dirección y supervisión del Dr. Antonio Manuel Martínez González, dando su conformidad a la misma la Comisión Académica, con la finalidad de que sea presentada ante la Comisión de Doctorado.

La Rama de conocimiento por la que esta tesis ha sido desarrollada es:

$\square$ Ciencias

$\square$ Ciencias Sociales y Jurídicas

XIngeniería y Arquitectura

En Cartagena, a 4 de junio de 2012

EL PRESIDENTE DE LA

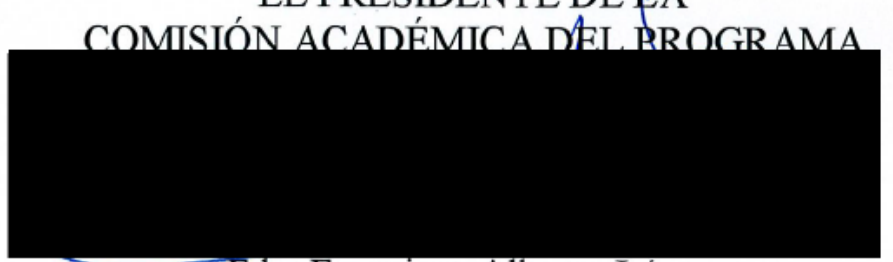

Fdo: Francisco Alhama López 



\section{Abstract}

Mobile communications have experienced a brutal raise over the past 15 years. What started as a voice communication system (GSM or $2 \mathrm{G}$ ) has finished yet as a data communication system of any kind, which in some cases has come to replace the conventional cabled data access infrastructure. This change in the use given to mobile devices necessarily entails a change in the underlying technology, which should be capable to provide the transmission speeds that these new applications require. This has emerged in recent years an increasing interest in multiple antenna techniques, usually referred as multiple-input multiple-output (MIMO) techniques, as they increase the spectral efficiency (and thus the transmission rate for a given bandwidth) of wireless systems.

In this thesis, some of the factors limiting the ideal advantages of these multiantenna techniques are studied, in order to quantify the differences between the ideal behavior of $4 \mathrm{G}$ devices and behavior that users will experience in actual use conditions. The effect that the user has on the final performance of the devices is one of the main limitations that these devices are in daily use. Mobile phones are used almost all the time in the vicinity of the user, causing a decrease in the richness of the multipath electromagnetic environment (and thus a reduction of the MIMO benefits). As a result of this reduction, the number of signal paths that reach the user is also reduced. In this thesis both factors (user influence and influence of the number of signal paths) will be studied both for passive devices (antenna prototypes) and active devices (commercial phones).

The second part of this thesis consist on the study of how to transfer some of these factors reducing the isotropicity of the environment, to one of the most promising measurement techniques, as it is the mode-stirred reverberation chamber (MSRC). This technique emulates naturally an isotropic rich multipath environment with the signal strength following a Rayleigh distribution. However, in this thesis two new techniques are proposed that allow the emulation of less isotropic environments without altering the basic operating principle of the MSRC. 
Measurement Techniques Enhancements for MIMO 4G Mobile Communication Systems.

Extension of Mode Stirred Reverberation Chambers (MSRCs) Emulation Capabilities 


\section{Acknowledgements}

This Ph.D. thesis has been developed in the Engineering Group of Microwaves, Radiocommunications \& Electromagnetism (GIMRE) of the Technical University of Cartagena (UPCT) in the years 2009-2012. I'd like to give my sincere thanks to Professor Antonio Martinez for supervising this thesis and for giving me his help whenever I needed during this time. His great knowledge and good judgment have been crucial for the successful outcome of this work. I would also like to thank Professor David Sanchez for their assistance in the development of this work and for the productive discussions we have had during this time, which have undoubtedly improved the work outlined in this thesis. Your expertise has been of great help to me, and I have learnt a lot from both of you throughout these years.

However, over the years, there have been many people who in one way or another, have contributed to the successful ending of this thesis. Special mention goes to my former lab colleagues Dr. Juan Francisco Valenzuela and Dr. Miguel Angel Garcia, with whom I spent a great time and I learned a lot about the fundamental issues developed throughout this work. My gratitude goes also to my current lab colleagues Francisco Clemente, Antonio Albero, and Rocío Murcia. Thank you all for making me much easier to develop this work. It was a real pleasure working with you.

I specially wish to thank Dr. Paul Hallbjörner and Thomas Bolin for their great support and help during the months I spent in Sweden, as well as for sharing their immense knowledge with me through all the very interesting discussions we had. I will always be indebted to you for giving me that opportunity. My gratitude goes also to the rest of the people I met at the EMC department at SP and the technology office at Sony Mobile, for all the significant help I had from them. I am looking forward to our future collaboration.

Finally, my most special gratitude goes to my family and friends for their invaluable support. To my parents, Fabian and Juana, for giving me all the opportunities they did not have, and for being always the best example both personally and professionally. To my brother Tony and sister Maria Ángeles for being my best friends and serve as inspiration in difficult times. And last but not least, to my girlfriend Maria José, for her endless support and understanding through all these years. This success is undoubtedly as much yours as mine.

This Ph.D. work was conducted under the financial support of the Spanish Ministry Of Economy and Competitiveness (former MICINN) through an FPI doctoral grant (BES2009-013764). 
Measurement Techniques Enhancements for MIMO 4G Mobile Communication Systems.

Extension of Mode Stirred Reverberation Chambers (MSRCs) Emulation Capabilities 


\section{List of Acronyms and Abbreviations}

3GPP 3rd Generation Partnership Project

ACK Acknowledge

ADG Apparent Diversity Gain

AoA Angle-of-Arrival

AoD Angle-of-Departure

AS Angle Spread

BER Bit Error Rate

BLER Block Error Rate

BPS Bits Per Second

BSE Base Station Emulator

CDF Cumulative Distribution Function

CRC Cyclic Redundancy Code

CTIA Cellular Telecommunications Industry Association

DG Diversity Gain

DS Delay Spread

DTX Discontinuous Transmission

DUT Device Under Test

EDG Effective Diversity Gain

EIS Effective Isotropic Sensitivity

EMC Electromagnetic Compatibility

FDD Frequency Division Duplex

FER Frame Error Rate

FFT Fast Fourier Transform

LoS Line-of-Sight

MIMO CE MIMO Channel Emulator

MIMO Multiple-Input Multiple-Output

MPC Multipath Components

MPS Multipath Simulator

MRC Maximum Ratio Combining

MSRC Mode Stirred Reverberation Chamber

NACK Negative Acknowledgement

NLoS Non Line-of-Sight

OTA Over-the-air

PAN Personal Area Network

PDF Probability Density Function

PDP Power Delay Profile

PIFA Planar Inverted F Antenna 


$\begin{array}{ll}\text { QAM } & \text { Quadrature Amplitude Modulation } \\ \text { QPSK } & \text { Quadrature Phase-Shift Keying } \\ \text { RC } & \text { Reverberation Chamber } \\ \text { RF } & \text { Radio Frequency } \\ \text { RMS } & \text { Root Mean Square } \\ \text { SAM } & \text { specific anthropomorphic mannequin } \\ \text { SAR } & \text { Specific Absorption Rate } \\ \text { SC } & \text { Selection Combining } \\ \text { SIMO } & \text { Single-Input Multiple-Output } \\ \text { SISO } & \text { Single-Input Single-Output } \\ \text { SNR } & \text { Signal-to-Noise Ratio } \\ \text { TCH } & \text { Traffic Channel } \\ \text { TE } & \text { Transverse Electric } \\ \text { TEM } & \text { Transverse Electric Magnetic } \\ \text { TIS } & \text { Total Isotropic Sensitivity } \\ \text { TM } & \text { Transverse Magnetic } \\ \text { TRM } & \text { Time Reversal Mirror } \\ \text { TRP } & \text { Total Radiated Power } \\ \text { UWB } & \text { Ultra Wide Band } \\ \text { VNA } & \text { Vector Network Analyzer } \\ \text { XPR } & \text { Cross-Polarization Ratio } \\ & \end{array}$




\section{Contents}

Abstract

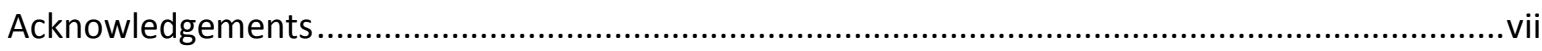

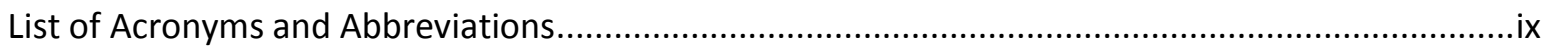

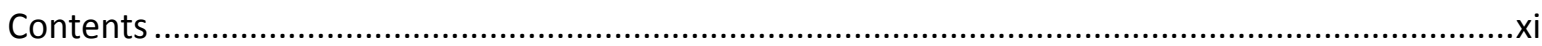

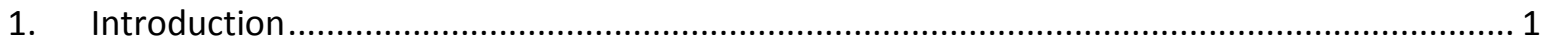

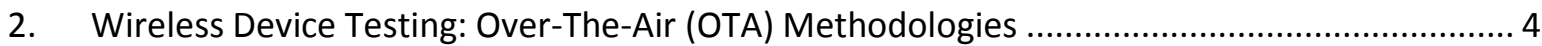

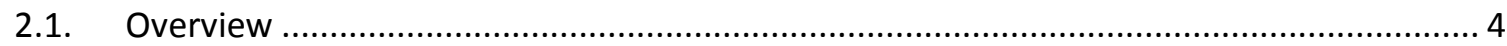

2.1.1. Single-Input Single-Output (SISO) Tests ……...................................................... 5

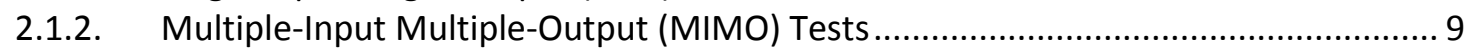

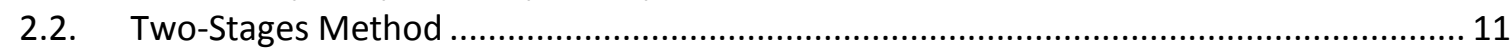

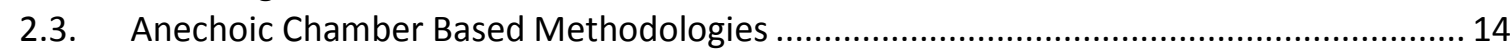

2.4. Mode-Stirred Chamber Based Methodologies .................................................................. 16

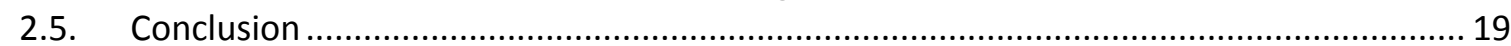

3. The Mode-Stirred Reverberation Chamber: Working Principles and Latest Advances ........... 21

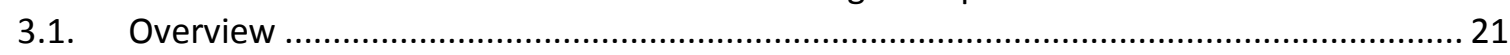

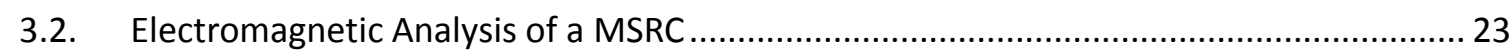

3.3. OTA Measurement Procedure Using a MSRC: The Rayleigh Distributed Environment.... 25

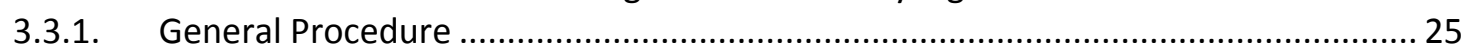

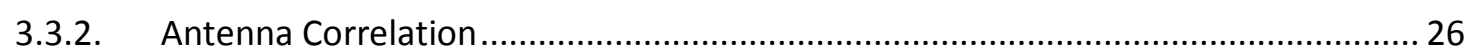

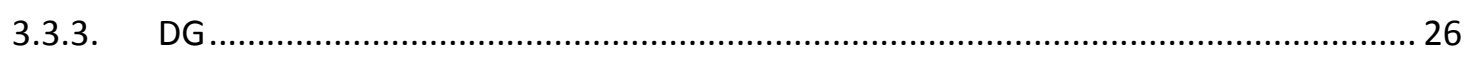

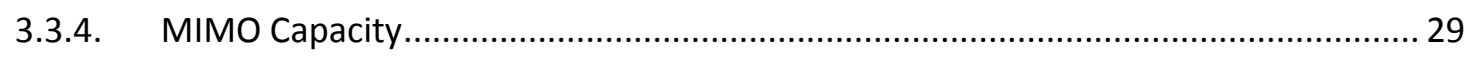

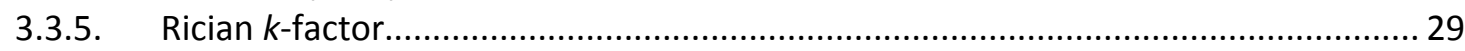

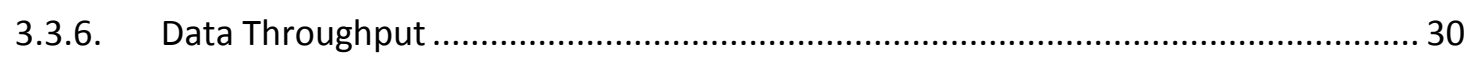

3.4. Latest Advances In Non-Isotropic Channel Emulation using MSRCs................................. 31

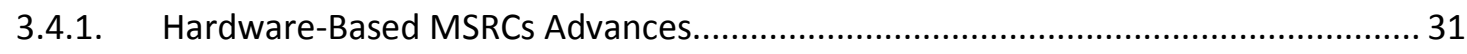

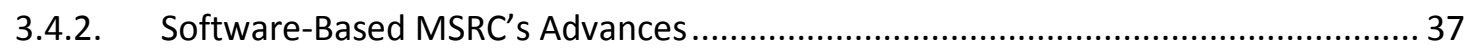

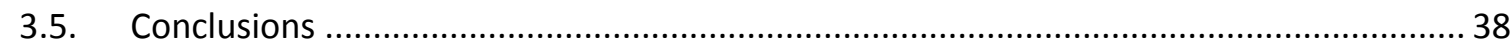

4. The MIMO Propagation Channel Model for Wireless Devices Testing …................................. 39

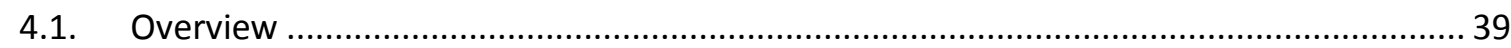

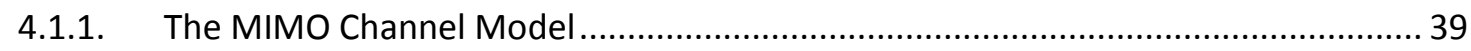

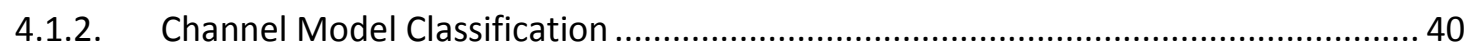

4.2. Standardized Channel Models Commonly Used in Industry ............................................. 42

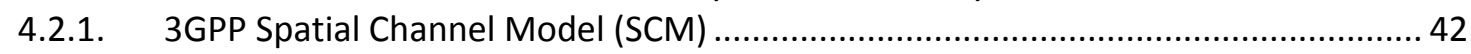

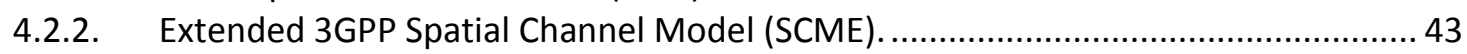

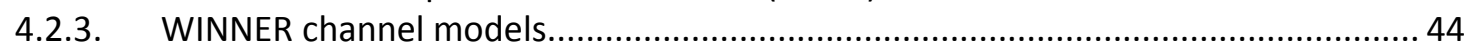

4.3. Most Relevant Channel Model Parameters for OTA Testing. .............................................. 47

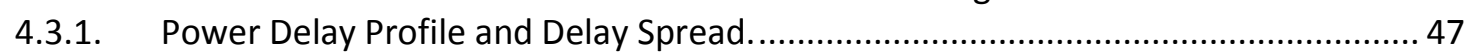

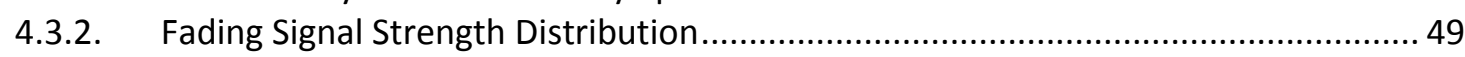

5. Study on the Effect of User Presence over Wireless Devices Performance.............................52

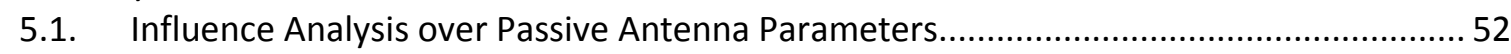

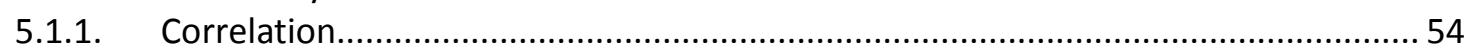

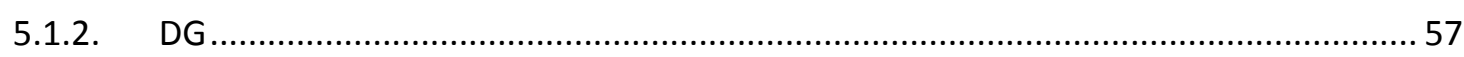

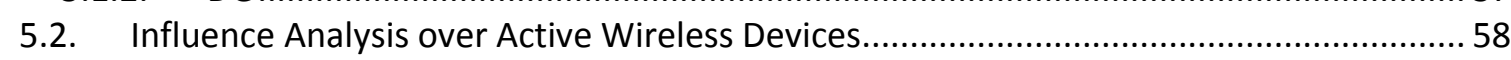

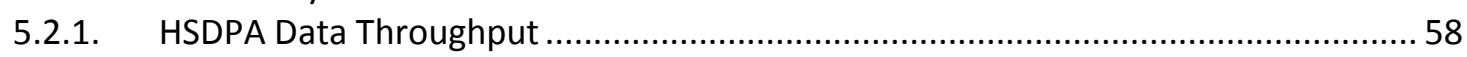

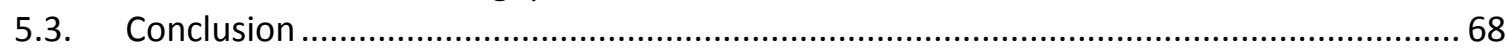

6. Study on the Effect of a Limited Number of Signal Paths Over Wireless Devices

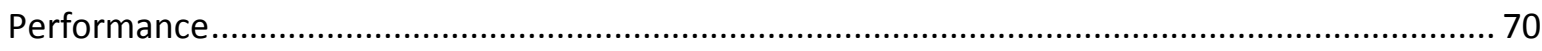

6.1. Influence Analysis over Passive Antenna Parameters.................................................. 70

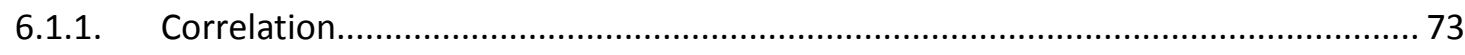

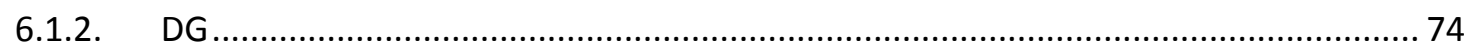


Measurement Techniques Enhancements for MIMO 4G Mobile Communication Systems.

Extension of Mode Stirred Reverberation Chambers (MSRCs) Emulation Capabilities

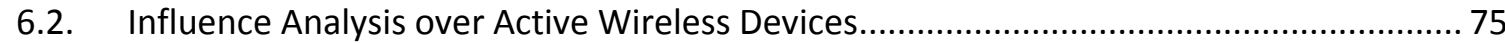

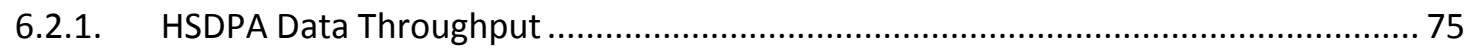

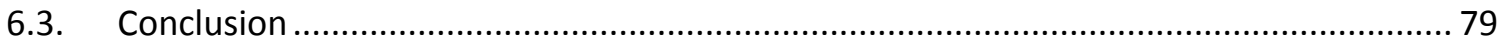

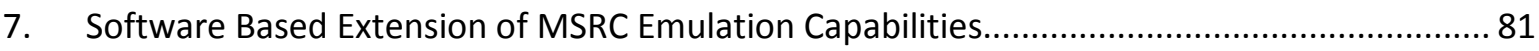

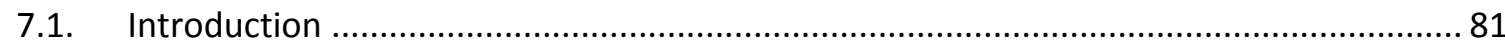

7.2. Arbitrary Rician Distributed Fading Emulation: The Offset Technique ............................ 82

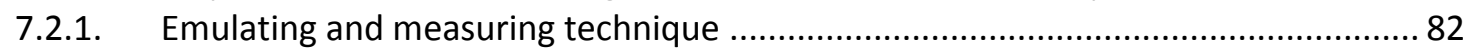

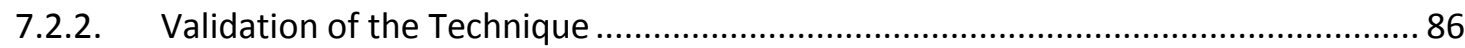

7.3. Arbitrary Fading Emulation: The Sample Selection Technique ........................................ 92

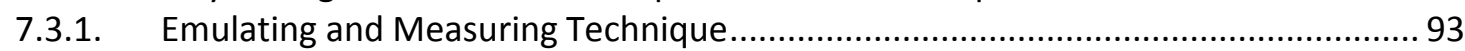

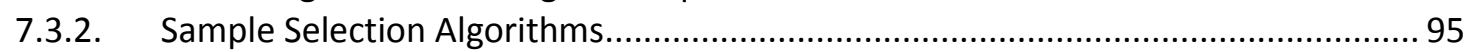

7.3.3. Limit for the Proportion of Remaining Samples..................................................... 98

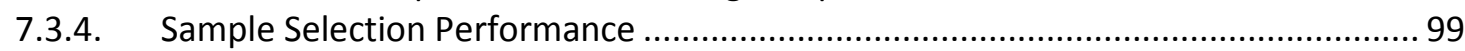

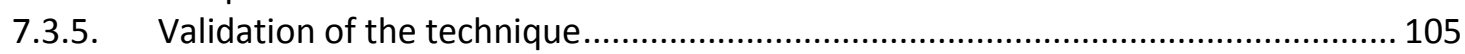

7.3.6. Sample-Selection Application to Other Channel Metrics....................................... 108

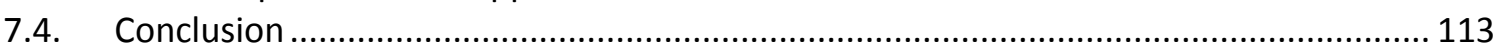

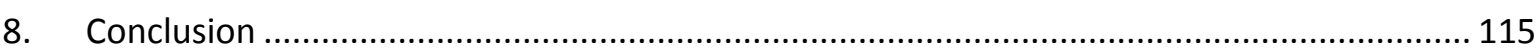

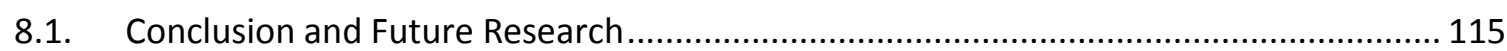

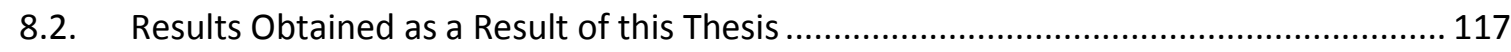

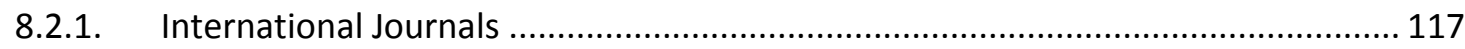

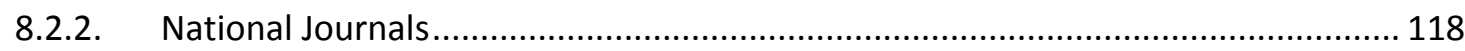

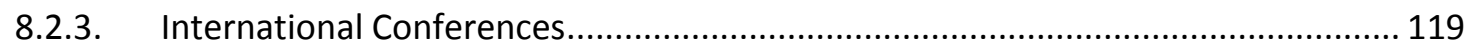

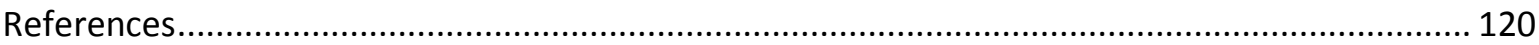

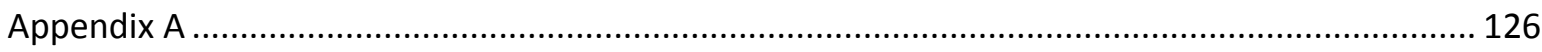

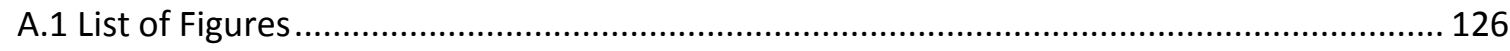

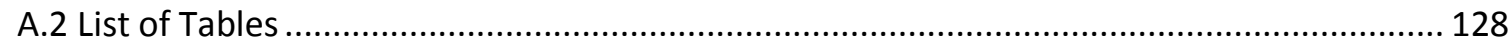




\section{Introduction}

The need for faster and faster data transmissions in wireless communication systems has caused a multitude of scientific studies in recent years. One of the most important for the evolution of these systems has been the introduction of multiantenna techniques, commonly called MIMO (Multiple-input Multiple-output). MIMO technology leverages multipath propagation experienced by a signal in real environments, originally seen as a problem, so that it is possible to establish communication channels parallel with the introduction of a diversity factor between transmitters and receiving antennas (polarization, radiation pattern...). This technology, ideally expected increases in channel capacity proportional to the number of antennas in the system for Rayleigh-distributed fading environments. However, the propagation environments experienced in actual use, are considered far from ideal. It is therefore crucial in the characterization of MIMO systems to have a correct emulation of the propagation environment in which the device under test (DUT) will be used.

In this thesis, some factors are studied that degrade the ideal capacity of MIMO systems in real environments. To do this, it is also studied in depth one of the most used measurement techniques, as they are the mode-stirred reverberation chamber (MSRC) based techniques, and new developments are proposed in order to extent the propagation environments that such techniques can emulate. Chapter 2 presents a brief overview of the various tests to which wireless devices are subjected before reaching the final consumer, and the various measurement techniques proposed for the characterization of MIMO systems. Chapter 3 discusses more about the technique of MSRC, analyzing the foundations of use, advantages and disadvantages. Also presented in this section is a summary of the various modifications proposed in the literature for the extension of the measurement capabilities of these systems. Chapter 4 presents a brief summary of the most used propagation environment models in the 
mobile communications industry as well as some of the main parameters used to define these models.

Chapter 5 examines the effect that the user presence has over some of the most important metrics in MIMO communication systems. To this end, several prototypes with pre-defined behavior (good and bad designs) are analyzed. Thus, it is intended to quantify the reduction of benefits that the user's presence can have on real $4 G$ prototypes.

Another factor that may limit the performance of a MIMO system is the number of signal paths that reach the user, when it is working in a real environment. Chapter 6 considers the effect of this factor on the same prototypes discussed in the previous chapter. The channel models used in industry (discussed in Chapter 4) assume a large number of signal paths, creating enough richness in the environment to take advantage of MIMO technology. However there are many actual environments where most of the transferred power is contained only in a few signal paths, which requires studying more in depth the effect of the number of signal paths over the MIMO metrics.

Finally, in Chapter 7 they are proposed two software processing techniques that allow the emulation of non-isotropic environments in MSRCs. MSRCs naturally emulate environments isotropic and Rayleigh-distributed, typical of urban conditions where a large number of multipath reflections are present. However, these environments do not reflect the reality of all scenarios where a wireless device is intended to work. Therefore, extending the measurement capabilities of MSRCs is crucial for the correct characterization of devices, when using this technique. Several advances have been proposed in the field of MSRCs in recent years, although almost all have involved the introduction of hardware alterations of the main cavity of the MSRC. However, this chapter proposes a different approach that exploits the statistical nature of the data obtained in a MSRC, by performing a data processing that allows the emulation of nonisotropic environments other than those conventionally emulated in a MSRC. First, it is proposed a technique to emulate Rician-distributed environments, which are the environments that are encountered when there is a component of sight between the transmitter and receiver (LoS condition). This technique is called "offset technique". Secondly, it is proposed a more ambitious technique (referred to as "sample selection"), which aims to expand the emulation capabilities of MSRCs so that any arbitrary fading distribution environment can be emulated. This technique is based on selecting only a subset of samples from the total measured samples (which together make a Rayleigh distribution) in accordance with an established pattern of fading. Thus, the measurements obtained considering only the selected samples have a 
pattern different from the Rayleigh distribution naturally generated in the MSRC, and is completely controllable by the user.

Finally, Chapter 8 presents conclusions and future research of this thesis, and a summary of the main results derived from this thesis in the form of journals and conferences papers. 


\section{Wireless Device Testing: Over-The-Air (OTA) Methodologies}

\subsection{Overview}

Every wireless device who reaches the market, and therefore the costumer, needs to pass a sort of tests that ensure that the device performance achieves the minimum performance established for the specific market where the device is going to work.

At the beginning, when the first mobile phone networks were deployed (early in the 90's), device tests were focused on guaranteeing that devices did not affect the electromagnetic environment (such as radio or television communications, electrical appliances...). These tests, called electromagnetic compatibility tests (EMC), are compulsory for every electronic device and they guarantee that devices do not emit spurious radiation which could be dangerous for any other device with electrical components, as well as that those devices are not disturbed by the effect of other electromagnetic radiation. Regulation is different for different territories. In Europe, manufacturers of electronic devices are advised to run EMC tests in order to comply with compulsory CE-labelling. The applicable regulation is the $\mathrm{EU}$ directive 2004/108/CE [1](previously 89/336/EEC).

Next step in wireless device testing came in 2001, when it was published the normative CENELEC EN 50360 and 50361 [2] [3] (substituted in 2005 by IEC 62209 [4]. This normative regulates the maximum permitted values of specific absorption rate (SAR), as well as the measurement procedure. SAR quantifies the rate at which the human body absorbs energy when it is exposed to radio frequency (RF) electromagnetic fields. It is measured in watts per kilogram, and usually averaged over a small portion of volume (1g or $10 \mathrm{~g}$ of tissue). SAR measurements are not performed using real people, instead a specific anthropomorphic mannequin (SAM) phantom filled with a tissue simulating liquid which has the same electrical characteristics that 
the human body for the frequency under test. The device under test (DUT) is then put attached to the SAM phantom simulating the typical positions which the devices is supposed to work in real use cases, and in a controlled radiation mode in such a way that the device is radiating at its maximum possible power. With that setup, the electric field is measured and averaged during 6 minutes, and the absorbed power is calculated. Limit values depend on the territory, and in Europe the limit for SAR calculated over the whole body is $2 \mathrm{~W} / \mathrm{Kg}$.

However the tests described in the previous paragraphs do not give us any information about the radiation performance of the DUT for communication purposes. In the first mobile phones, antennas were usually a syntonized external dipole, so antenna performance was similar for many of the available mobile phones. Later on, when antenna designs became more complex, differences between mobile phones antenna performance became bigger and, therefore, differences on the service quality experienced by the user was also different. This phenomenon had an important impact for telecommunication operators, since they are the final responsible of the service quality experienced by their users, and it raised the necessity of evaluating the device antenna performance to assure that every device put in the market is able to give a minimum performance to the user in terms of coverage and communication quality.

\subsubsection{Single-Input Single-Output (SISO) Tests}

As part of this increasing interest the Cellular Telecommunications Industry Association (CTIA) released in 2001 its document "Test Plan for Mobile Station Over the Air Performance" (currently released version 3.1 [5]). This document defines general requirements for equipment configurations, laboratory techniques, test methodologies, and evaluation criteria that must be met in order to ensure the accurate, repeatable, and uniform testing of wireless devices. Proposed metrics for device radiation performance evaluation are "Total Radiated Power" (TRP) and "Total Isotropic Sensitivity" (TIS).

TRP is a metric that provides information about the radiated RF performance of the DUT. The procedure to measure TRP consist of measuring the radiated transmit power at various locations surrounding the device in a three-dimensional way, and taking samples every 15 degrees in the Theta $(\theta)$ and in the Phi $(\phi)$-axes. This accounts for a total of 264 measurements for each of two orthogonal polarizations since measurements at Theta $=0$ and 180 degrees are not required. All of the measured power values will be integrated to give a single figure of merit.

The total radiated power from a cellular phone depends on how much power is conducted from power input to the antenna, and how good the antenna is at 
transforming that to radiated power in other words, the antenna's efficiency. Efficiency is greatly dependent on how materials absorb radiated power, and since the typical usage scenario of wireless devices requires the device to be held close to a human head, the antenna efficiency will be different in a position close to human tissue. This means that characterizing an antenna using efficiency typically requires free-space efficiency and an efficiency measured next to a standardized phantom head. A good example is a wireless carrier requiring a handset to meet a TRP of +22 $\mathrm{dBm}$ in the GSM850 band when the device is held next to a head. Since the nominal conducted power in GSM850 is $+33 \mathrm{dBm}$, the antenna efficiency and the changes caused by the head are allowed to cause a degradation of $11 \mathrm{~dB}$ and still be able to meet the TRP number

Sensitivity measurements in wireless communications seek to determine the cell phone's ability to receive low signals. Performing these measurements is an iterative process which varies the base station (BS) output power at the phone while measuring the Bit-Error-Rate (BER), the Frame Erasure Rate (FER) or the Block Error Rate (BLER), depending on the system. When a target BER/FER is achieved, the iteration is stopped and the output power at the phone is recorded as the sensitivity (i.e., the minimum power required to maintain a specified BER/FER). Sensitivity is measured by lowering the BS Traffic Channel (TCH) power level until the specified digital error limit is exceeded (for example, $1.2 \%$ of BER for UMTS). The TCH power that was required to obtain the error limit is the sensitivity value. To do this, the BS is placed in loop-back mode. The BS transmits a bit pattern to the phone and the phone transmits it back. The returned bit pattern is then compared and the BER/FER/BLER is determined. As the output power from the BS to the phone is reduced, the BER/FER/BLER increases. Therefore, TIS is a figure of merit of overall radiated sensitivity of a wireless terminal. Measurements are taken every 30 degrees in the Theta $(\theta)$ and in the Phi $(\phi)$-axes in order to have a three-dimensional evaluation, which give us a total of 60 measurements. All of the measured sensitivity values for each DUT test condition will be integrated to give a single figure of merit. It is calculated as the integral of the measured Effective Isotropic Sensitivity (EIS).

Both TRP and TIS are commonly accepted over the world as the Figure of Merit (FoM) to properly characterize devices using only one antenna (SISO). This is mainly due to the simplicity of the measurements, as well as that both of them give us an intuitively idea about how good or bad the device is working in both transmission (TRP) and reception (TIS). Most of the work in this field was then concentrated on defining the right measurement procedure, the uncertainty of the technique and the minimum 
DUT performance demanded. The maximum uncertainty allowed is defined in [5], and showed in Table 1.

TAble 1. EXPANded UnCertainty MaXimum Limits (IN DB) FOR DifFerent Configurations for TRP AND TIS (SOURCE: [5])

\begin{tabular}{c|c|c}
\hline \hline Test Configuration & TRP & TIS \\
\hline Free Space & 2 & 2.3 \\
Beside Head & 2.1 & 2.3 \\
Beside Head and Hand Right & 2.4 & 2.6 \\
Hand Right & 2.2 & 2.4 \\
\hline
\end{tabular}

As it is shown in Table 1, measurements of TIS and TRP can be done in four different use cases. These different cases simulate the most common scenarios where the DUT is supposed to work, including the effect that user presence has over the device performance. The four typical measurement scenarios are:

- $\quad$ Free-Space: where the DUT is placed on a support made of low dielectric material in order to avoid any effect from the close environment. This case is not realistic, but it is very useful as a reference case for intercomparison purposes.

- $\quad$ Head Phantom Only: where the DUT is placed against a head phantom.

- Head and Hand Phantom: where the DUT is placed in a hand phantom, against a head phantom. This position is commonly known as "talk" position, and it gives an accurate idea about the device performance in a realistic case.

- Hand phantom only: where the DUT is placed in a hand phantom. This scenario (using one or two hands) is commonly known as "browse" mode, and it simulates the situation where the user is using the device to send and receive data (SMS, Internet data...).

However, when it comes to the definition of the minimum DUT performance demanded it is not easy to have agreement. In one hand operators want to set high performance levels, to be able to give coverage to their users reducing to the minimum the amount of base stations installed on the street. On the other hand, mobile phone manufacturers want to keep the requirements as lowest as possible to avoid that old devices were catalogued as "no compatibles" and also to have some margin on the 
design due to the tendency of reducing the size of the phones, which is a big inconvenient for the antenna performance at low frequencies. The solution was a compromise between manufacturers and operators. The 3GPP document TS 34.114 [6] defines the minimum and average TRP and TIS (referred as TRS in all 3GPP documents), but it also defines a recommended performance which is not compulsory but it is showed as a goal for the manufacturers. Examples of minimum and average values are extracted from [6] and shown in Tables 2 and 3 for Frequency-Division Duplexing (FDD) systems (such as W-CDMA). Recommended values are about 2 or $3 \mathrm{~dB}$ better than the demanded average values.

TABLE 2. TRP MINIMUM PERFORMANCE REQUIREMENT FOR FDD ROAMING BANDS IN THE SPEECH POSITION AND THE PRIMARY MECHANICAL MODE. (SOURCE: [6])

\begin{tabular}{c|c|c|c|c|c|c}
\hline \multirow{2}{*}{$\begin{array}{c}\text { Operating } \\
\text { band }\end{array}$} & \multicolumn{2}{|c|}{ Power Class 3 } & \multicolumn{2}{c|}{ Power Class 3bis } & \multicolumn{2}{c}{ Power Class 4 } \\
\cline { 2 - 7 } & \multicolumn{2}{|c|}{ Power (dBm) } & \multicolumn{2}{c}{ Power (dBm) } & \multicolumn{2}{c}{ Power (dBm) } \\
\cline { 2 - 7 } & Average & Min & Average & Min & Average & Min \\
II & +15 & +13 & +15 & +13 & +13 & +11 \\
III & +15 & +13 & +15 & +13 & +13 & +11 \\
IV & +15 & +13 & +15 & +13 & +13 & +11 \\
V & +11 & +13 & +15 & +13 & +13 & +11 \\
VI & +11 & +9 & +11 & +9 & +9 & +7 \\
VII & +15 & +13 & +15 & +13 & +13 & +11 \\
\hline \hline
\end{tabular}


TABLE 3. TRS MINIMUM REQUIREMENTS FOR FDD ROAMING BANDS IN THE SPEECH POSITION FOR THE PRIMARY MECHANICAL MODE. (SOURCE: [6])

\begin{tabular}{c|c|c|c}
\hline \hline Operating Band & Unit & Average & Max \\
\hline II & $\mathrm{dBm} / 3.84 \mathrm{MHz}$ & -101 & -98 \\
III & $\mathrm{dBm} / 3.84 \mathrm{MHz}$ & -99 & -96 \\
IV & $\mathrm{dBm} / 3.84 \mathrm{MHz}$ & -98 & -95 \\
V & $\mathrm{dBm} / 3.84 \mathrm{MHz}$ & -101 & -98 \\
VI & $\mathrm{dBm} / 3.84 \mathrm{MHz}$ & -96 & -93 \\
VII & $\mathrm{dBm} / 3.84 \mathrm{MHz}$ & -96 & -93 \\
\hline \hline
\end{tabular}

\subsubsection{Multiple-Input Multiple-Output (MIMO) Tests}

In the previous section we have seen that the performance of a SISO DUT can be easily characterize through a couple of power based metrics, one standing for the transmitting performance and the other one standing for the receiving characteristics. This is commonly accepted, because in SISO communications the relation between performance and power level is linear. In an environment with a fixed noise level, more received power univocally means better signal quality. But it is not true when more than one antenna is used.

MIMO systems have more than one antenna in both ends of the communication, so they are able to maintain multiple data streams at the same time. The main idea in MIMO is to take advantage of the spatial diversity that the receptor experience due to the multipath propagation environment found in real use conditions. Spatial diversity can be quantified in terms of signal correlation between the receiving antennas, and it is a function of both propagation environment and antenna correlation. In ideal conditions a MxN MIMO system (with $\mathrm{M}$ transmitting antennas and $\mathrm{N}$ receiving antennas) has an ergodic increase of data rate of $\min \{M, N\}$ times the data rate of the corresponding SISO case [7]. Ideal conditions are an environment where the signal correlation at the receiving point is zero (completely uncorrelated signals at RX point), completely uncorrelated receiving antennas, and a static propagation channel which allows the receptor to have the correct information about the channel. But the propagation environments typically found in the real world are not even close of those ideal conditions. 
In fact, MIMO devices are especially sensitive to factors like the propagation environment, antenna design and orientation and baseband algorithms. This makes impossible to predict the final behaviour of a MIMO device from the power values transmitted or received, and it becomes necessary to found a different FoM from TRP and TIS. The FoM that is nowadays accepted as a good indicator of how good or bad a DUT works is the data throughput, which stands for the total amount of bits transmitted correctly from the transmitter to the receptor. In Table 4 are showed the currently FoM under study, and the methodology how they must be measured (with or without fading)

TABle 4. CATEgories of Figure of Merits

\begin{tabular}{|c|c|c|c|c|c|}
\hline \hline Category & I & II & III & IV & V \\
\hline \multirow{4}{*}{ FOMs } & $\begin{array}{c}\text { MIMO } \\
\text { Throughput } \\
\text { Type }\end{array}$ & $\begin{array}{c}\text { TRP } \\
\text { CQI }\end{array}$ & $\begin{array}{c}\text { Gain Imbalance } \\
\text { Spatial } \\
\text { correlation } \\
\text { MRS }\end{array}$ & $\begin{array}{c}\text { Antenna } \\
\text { Efficiency } \\
\text { MIMO Capacity }\end{array}$ & $\begin{array}{c}\text { MIMO } \\
\text { Throughput } \\
\text { (VRC) }\end{array}$ \\
& OTA & OTA & MIMO antennas & MIMO antennas & OTA \\
Active & $\begin{array}{c}\text { Active } \\
\text { (with fading) }\end{array}$ & $\begin{array}{c}\text { Passive/Active } \\
\text { (with fading) }\end{array}$ & $\begin{array}{c}\text { Passive/Active } \\
\text { (without fading) }\end{array}$ & $\begin{array}{c}\text { Active } \\
\text { (with fading) }\end{array}$ \\
\hline \hline
\end{tabular}

MIMO Throughput, as it is defined in [8] is calculated from the block error rate (BLER) measured value, and it stands for the time-averaged number of correctly received transport blocks by the DUT. The MIMO Over-the-air (OTA) throughput is measured at the top of physical layer of wireless systems. The Base Station Emulator (BSE) calculates both BLER and downlink throughput as follows,

$$
\begin{gathered}
\text { Throughput }(\%)=100-D L B L E R \\
\operatorname{DLBLER}(\%)=\left[1-\frac{N A C K+D T X}{A C K+N A C K+D T X}\right] \times 100
\end{gathered}
$$

Where,

- ACK: percentage of transmission packets that the UE answered after successful Cyclic Redundancy Code (CRC) check.

- $\quad$ NACK: percentage of transmission packets that the UE answered to indicate unsuccessful transmission.

- DTX: percentage of transmission that were not answered at all and caused discontinuous transmission. 
The time over which the MIMO throughput measurement is averaged must be long enough to correctly average the values over the fading variations.

A standardization process is being carried out by the $3^{\text {rd }}$ Generation Partnership Project (3GPP) organization (in parallel with CTIA), in order to define the propagation environments where the DUT must be tested as well as the MIMO configuration used in those environments. The options are almost infinite, and a lot of discussion has been done during the last few years. The result is synthesized in the 3GPP document TR 37.976 [8], and some of the important points still are not clear.

One of the most important parts of the standardization process is the definition of the measurements techniques which will be considered to be able to emulate the defined propagation models. Several techniques are still under study, and each one has some advantages and disadvantages. The different methodologies differ in terms of complexity and cost-effectiveness. In order to adequately evaluate the overall MIMO performance of mobile terminals equipped with multi-antennas for the receive diversity and MIMO transmission, several figures of merit have been proposed. These techniques can be grouped in three main categories: Two-Stages Method, Anechoic Chamber based, and MSRC based.

\subsection{Two-Stages Method}

The two stages method is based on the assumption that all the necessary information for antenna characterization is contained in the far-field antenna radiation pattern. Therefore the method divides the MIMO OTA tests in two separate measurements. Firstly, the MIMO antenna radiation pattern is measured inside an anechoic chamber. Secondly, a commercial MIMO Channel Emulator (MIMO CE) is used to mathematically convolve the measured antenna characteristic with the chosen MIMO OTA channel model for real time emulation.

The first stage consists of the characterization of the three dimensional antenna radiation patterns of all the antennas in the DUT, using an anechoic chamber. The setup needed to perform this part of the measurement is defined in the 3GPP document TS 34.114 [6]. The DUT is placed in a chamber and the far zone radiation patter of each antenna element is measured in free space, for the two orthogonal polarizations (typically linear theta $(\theta)$ and phi $(\phi)$ polarizations as shown in Fig. 1). User influence can be included in this stage, by repeating the antenna radiation pattern characterization including head and hand phantoms. If the DUT has dynamic antenna tuning elements, detailed information on the implementation is required to understand the consequences for the pattern measurement. 


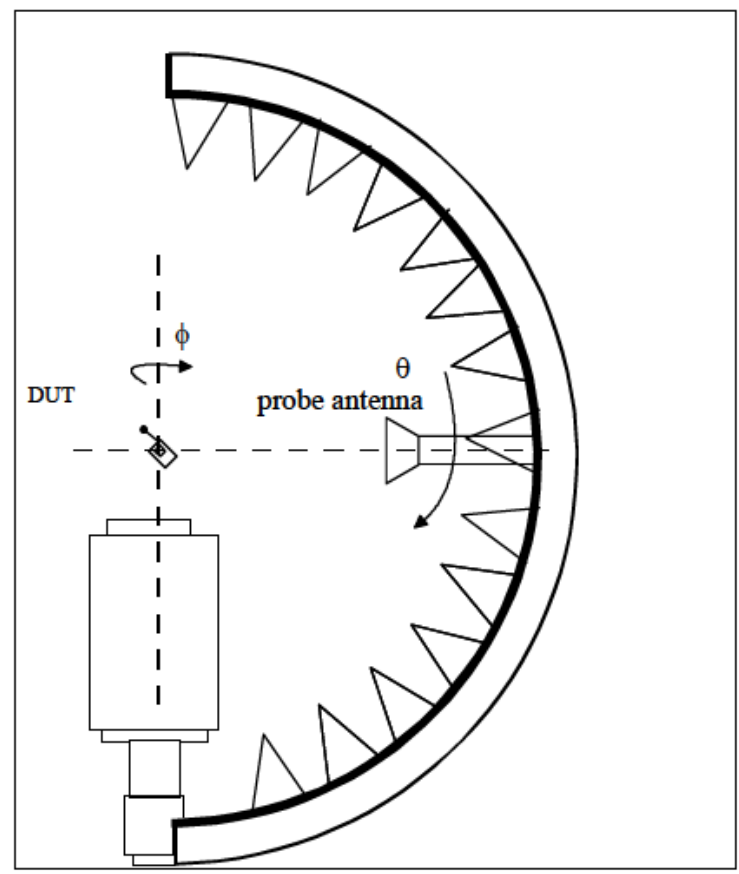

Figure 1. The coordinate system used in the measurements. SOURCE: [8]

The second stage of this method consists on performing the desired FoM conducted measurements. To establish the communication, the BSE is connected to the MIMO CE (emulating the compound channel, including both the MIMO antenna effect and the multipath channel effect) and then to the MIMO DUT's temporary antenna ports via approved RF cables, as it is showed in Figure 2. These ports are the standard ones provided for conducted conformance tests. By controlling the power settings of the channel emulator and also the integrated channel model, the end-toend throughput with the MIMO antenna radiation influence can be measured. 


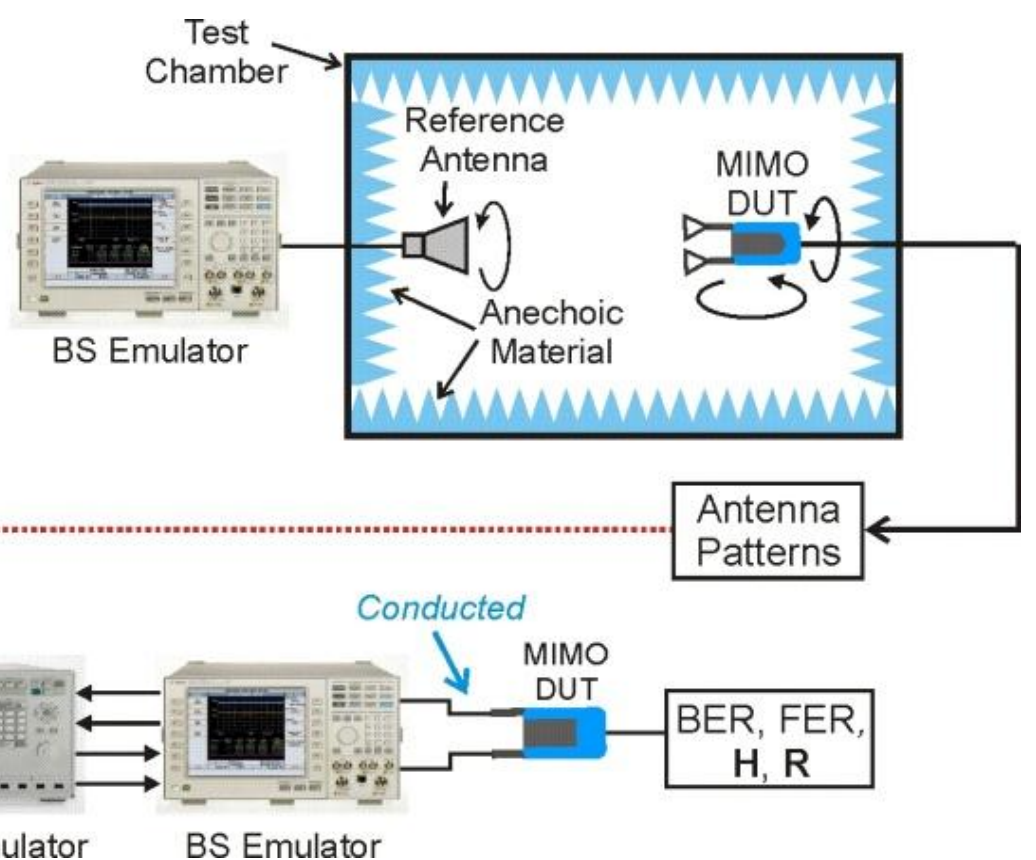

Channel Emulator

BS Emulator

Figure 2. Proposed two-stage test methodology for MIMO OTA test. SOURCE: [8]

The two-stage method has some advantages respecting to other solutions [9].

- The method can reuse existing SISO OTA anechoic chambers to make the antenna pattern measurements; only a channel emulator with a number of outputs, two or four in the most common cases is required (to match the number of device receiver inputs) regardless of the complexity of the chosen channel model.

- This method is therefore easily scalable to higher order MIMO due to the reduced number of instruments required, compared some of the other solutions.

- The implementation of the channel models is very accurate and flexible due to being implemented electronically, and it can be modified to fit any desired operating conditions such as indoor-outdoor, high or low Doppler spread, high or low delay spread, beam width, in 2D or full 3D etc.

On the other hand, some disadvantages of the method can be listed as well.

- $\quad$ The first is the impossibility of directly measure the desensitization created by the eventual signal leakage from the device transmit antennas into the receiving antennas, since the antenna pattern measurement does not take account of that leakage. This phenomenon is however measured in existing SISO OTA receiver sensitivity measurements.

- The second disadvantage is the necessity of the DUT's chipset to support amplitude and relative phase measurements of the antennas. 


\subsection{Anechoic Chamber Based Methodologies}

The second MIMO OTA test methodology groups a number of slightly different methodologies, but all of them include an anechoic chamber and several antennas that surround the DUT. These techniques are commonly referred as anechoic chamber based methodologies.

These methodologies consist of a large number of probe antennas placed inside an anechoic chamber transmitting with temporal and spatial characteristics for testing multiple antenna devices. Probe antennas are positioned around the DUT in such a way that is possible to change the receiving spatial profile of Angle of Arrival ( $A \circ A)$ and consequently the Angle Spread (AS) at the DUT position. Spatial characteristics of the BS (Angle of Departure (AoD), such as correlation, delay, fading profile or Doppler shift) can be implemented using a feeding network (where the input signal is processed) or with a commercial channel emulator.

This OTA method approximates the MIMO channel using a geometry-based channel model such as the 3GPP SCM [10], that will be studied deeper in Chapter 3. Several probe antennas are usually grouped to create clusters in the compound signal at the DUT position. Each antenna probe (with its specific delay, Doppler shift...) emulates one subpath component of the cluster. In Figure 3 it is shown an example of the setup needed to implement this method, using 8 probe antennas and a channel emulator.

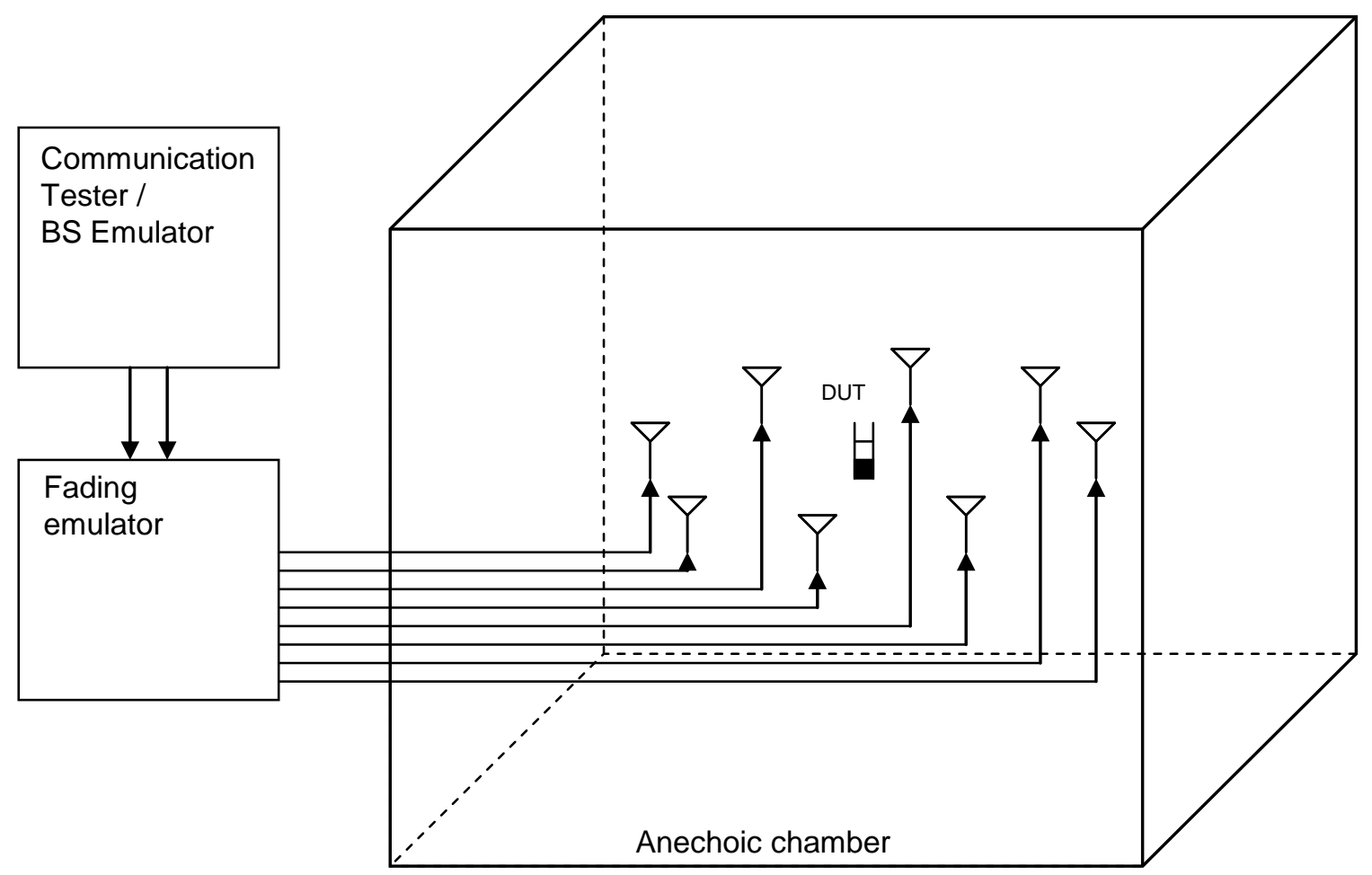

Figure 3. Example of MIMO/Multiantenna OTA test setup (Uplink signal path omitted in the figure) 
The number and distribution of the probe antennas will be fixed in a specific system, and it will determine the MIMO channel models that can be emulated. This highlights the importance of defining the desired channel models when the probe antennas distribution is being defined. For example, according to the different channels defined by the SCM [10], SCME (a SCM bandwidth extended version) [11], Winner I \& II [12] [13] models, the optimum number of probe antennas oscillates between 6 and 8 [8] for a given polarization. In general, the most flexible configurations require the higher number of probes. Elevation spread may be created by installing probes at different elevations, however doing this tends to constrain their flexibility.

This methodology provides flexibility in the number of probe antennas, so the solution is easily scalable. There is no upper limit and the lower limit is one (for single cluster channel models). The key question is how accurately the channel model is emulated. Based on the quiet zone discussion, it was proposed to use 8 antennas in the case of single polarization and 16 antennas in the case of dual polarization. On the other hand, if elevation is needed, the antenna number will be higher. Additionally, the antenna positions can be adjusted to optimize the accuracy with limited number of antennas.

Summarizing, the advantages of this method are:

- Measurements can be performed in an end-to-end way. On the contrary to the two-stage method, measurements with anechoic chamber method allow to actually measure the DUT as one complete system, including the antenna effect.

- $\quad$ Flexibility to emulate all the important parameters that characterize a channel model (Doppler shift, AoA, AoD, delay and polarization).

On the other hand, the main disadvantage of this methodology is the growing complexity and cost when the number of probe antennas grows, compared to the other solutions.

During the work performed on this thesis, one specific implementation of this methodology is used. The system is referred as multipath simulator (MPS). The MPS used during this work surrounds the test object with an array of antennas, where each array antenna represents one signal path. Each signal path has a certain polarization, power level, Doppler shift, and delay. The MPS is placed in a shielded room with absorbers on the walls in order to avoid unwanted disturbance. The test object is placed on a foam block at the centre of the array. During measurements, different Doppler shifts are applied to all array antennas, thereby ensuring fast fading conditions 
at the test object. It is designed specifically for mobile phone tests. Its antenna array comprises eight vertically and eight horizontally polarized antennas in a circle with 1.4 $\mathrm{m}$ radius.

In order to change the angular distribution of delays at the test object position a switch matrix is included in the MPS feeding network. The switch matrix is composed of a number of $2 \times 2$ switches, configured in two $4 \times 4$ switch matrices each with 24 possible settings. This way, it is possible to have $24 \times 24=576$ different angular distribution of delays, without changing the physical position of the phone. Each one of the output signals passes through a phase shifter (which introduces Doppler shift), an attenuator (which allows controlling the power of each cluster) and a power splitter (in order to feed two orthogonal polarized antennas). By controlling the phase of each RF signal, a Rayleigh distributed or other relevant multipath distribution can be obtained.

\subsection{Mode-Stirred Chamber Based Methodologies}

A typical MSRC, also referred as Reverberation Chamber (RC), consists of a closed metal cavity equipped with movable metal blades that act as field mixers (commonly called stirrers) and a turntable that rotates the DUT. A scheme is provided in Figure 4.

The independent movement of the stirrers and the rotation of the sample, allow to dynamically changing the boundary conditions of the electromagnetic field generated inside the cavity.

Figure 4. Mode-stirred chamber setup for devices testing with single cavity. SOURCE: [8]

In this way, the multimodal electromagnetic environment generated naturally in the cavity is agitated. Through this continuous agitation the distribution of the measured fields is independent of the sample position inside the cavity, as long as they respect a minimum distance of $\lambda / 2$ with respect to the cavity walls. For a complete uniformity of the generated field, it is also required that the polarization of the excited 
modes is uniform, which is achieved by using multiple transmit antennas with different polarizations [14].

The standard deviation of the measured field inside the cavity is typically used as the figure of merit that distinguishes good performance of MSRC. In a perfectly agitated MSRC, the real and imaginary parts of the rectangular components of electric and magnetic fields follow Gaussian distributions with identical paths variance. Thus, the amplitude of the electric and magnetic fields measured in a perfectly stirred MSRC follows a Rayleigh probability density function (PDF), while the phase is uniformly distributed. This type of distribution is very similar to the multipath field distribution found in urban environments for mobile communication systems.

Another interesting feature of this technique is that if we assume that the introduction of an adaptive antenna does not disturb the field distribution inside the cavity, it can be shown that the power received by this antenna adapted within the MSRC is independent of its gain, directivity and effective area [15].

This property, together with the repeatability and reliability of the stochastic distribution of the fields emulated inside the MSRC, make the MSRC's an emerging technology in the field of action OTA for both research and development and the certification of wireless terminals.

The naturally emulated Rayleigh environment in a MSRC is well known as a good reference for urban and indoor multipath environments, but does not reflect the reality of other propagation environments. However, most of the traffic generated by mobile phones is usually made indoors and in urban areas which can be very well represented by the MSRC. Therefore, it makes sense to use MSRCs for optimizing and evaluating devices with both single and multiple antenna configurations to be used indoors and in urban areas.

Limits to emulate arbitrary channel models in a typical MSRC can be summarize as,

- $\quad$ The Power Delay Profile (PDP) is limited to a single decaying exponential and therefore the Delay Spread (DS) cannot be arbitrarily set.

- The Doppler spectrum and maximum Doppler is limited by the relatively slow motion of the stirrers.

- It is difficult to impart a specific, repeatable MIMO fading correlation on the downlink waveform.

On the other hand, there are some important advantages that has become this methodology in an emerging technique during the last few years, 
- $\quad$ Cost effective solution: the number of probe antennas used in a MSRC is much lower than in other solutions (as those based in anechoic chamber).

- Measurement time: due to the capability of MSRC to emulate a 3-D environment (instead of 2-D environments generated with other methodologies), the measurement time with this technique is lower than in other techniques and can be even suitable to be included in production lines.

Notwithstanding that inherent limitation, many papers during the last years have focused their attention on introducing modification to the simple MSRC design, in order to emulate more complex channel models. Some of these advances will be discussed in Chapter 4 of this thesis more precisely. However it will be introduced here one of the most promising techniques proposed, which consist on adding more than one metal cavity so the fields do not necessarily have to be constrained to a single cavity. In consequence, MSRCs may contain more than one metal cavity that could be coupled through a variety of means, including waveguides, slots or metal plates, as it is shown in Fig 5.
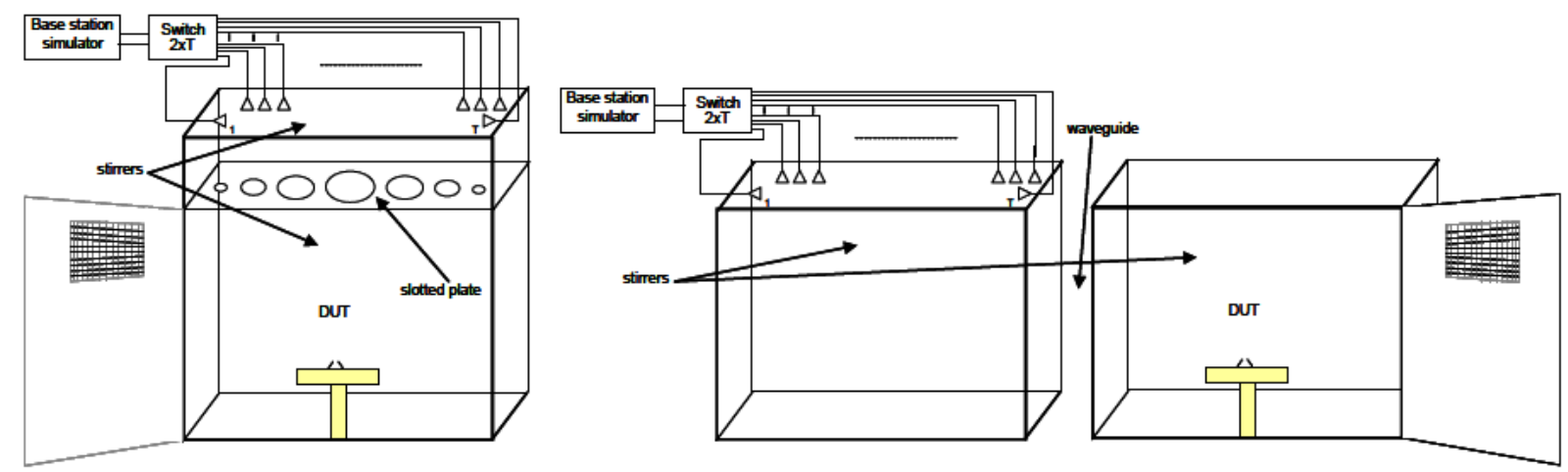

Figure 5. Mode-stirred chambers with multiple cavities. SOURCE: [8]

Another solution that has been proposed to overcome that limitation, consist of cascading a MIMO CE and the MSRC. Using that setup, the properties of an arbitrary channel can be set in the channel emulator, extending the channel model emulation capabilities of a typical MSRC. However, the channel models parameters are changed by the innate response of the MSRC, and therefore the signal properties inside the MSRC is not exactly the same than those set in the channel emulator.

An example of that effect is the way that the PDP of a given channel model is changed by a MSRC. A MIMO CE can be programmed with some fading taps at the desired excess delays, in order to emulate an arbitrary PDP. However, the resulting PDP inside the MSRC is the convolution of the desired PDP and the innate exponential decay response of the MSRC (that depends on the shape and size of the MSRC). 
Therefore, the PDP actually generated inside the MSRC is different from the desired one, and that difference must be studied.

In [16] this effect is studied when the "urban macro" SCME channel model is injected in a conventional MSRC. In Fig. 7, it is clearly appreciated the differences between the ideal PDP specified for the "urban macro" channel model (top plot of the figure), and the actual PDP measured inside the MSRC (bottom plot).
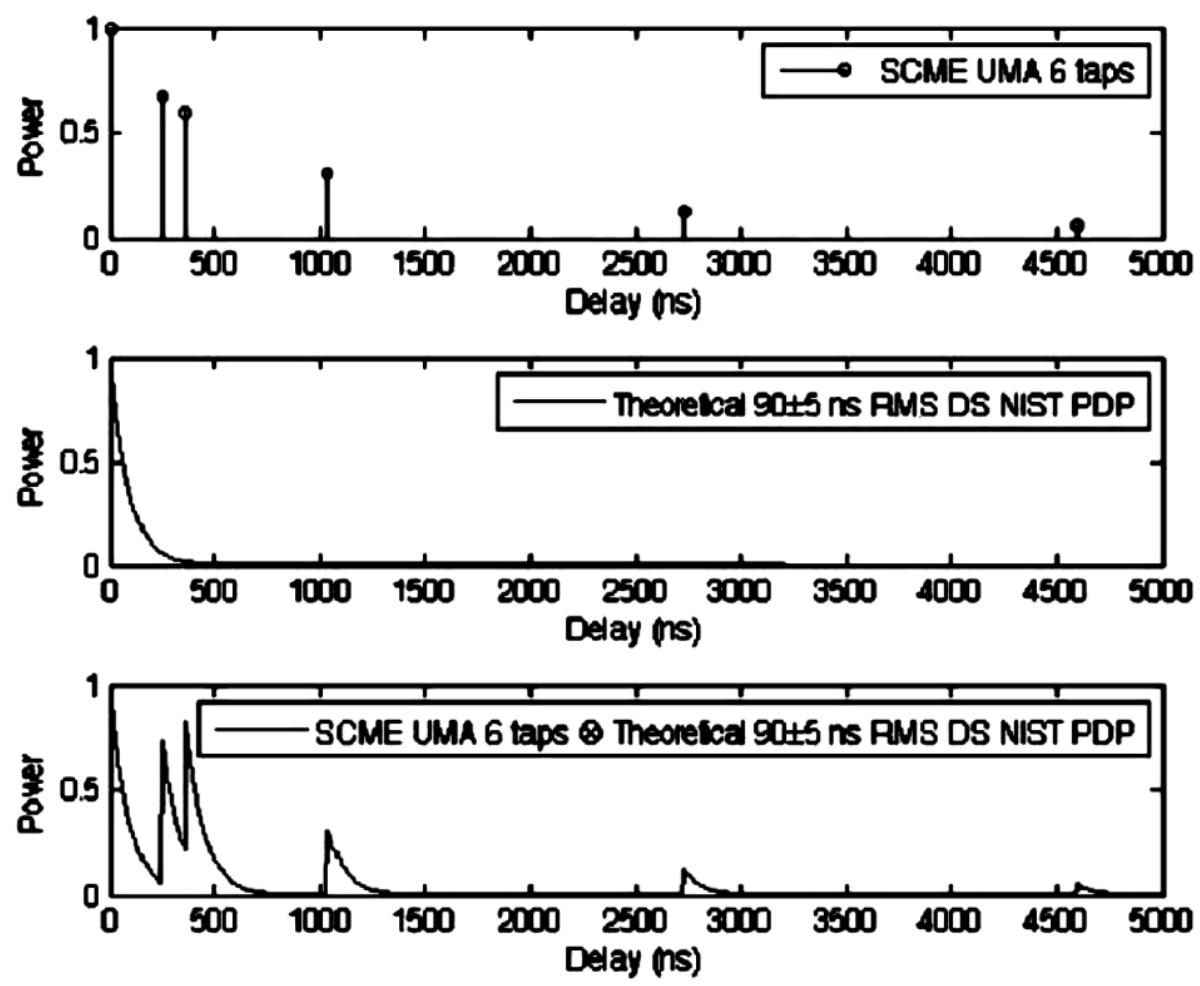

Figure 6. PDP convolution effect observed when a CE and a MSRC are cascaded.

\subsection{Conclusion}

The OTA measurement methods arise from the need to properly characterize the performance of wireless devices coming to market, so as to ensure a performance to guarantee that the network deployment is done efficiently (minimizing the number of base stations and transmitted power), but maintaining reliable communication.

The characterization of wireless systems was well defined in SISO systems with a single antenna (technology implemented in standards GSM, UMTS, HSPA) by the evaluation-of power levels based FoM's (TRP, TIS) of both transmitted and received power from the DUTs. However with the advent of multiantenna technology communication standards (HSPA +, WiMAX, LTE) the situation changes and the degree of complexity is increased.

The FoM's used are no longer directly proportional to the power, but evaluating the ultimate capacity of the system to send data correctly (end-to-end throughput). 
The throughput experimented by the end user takes into account all parts of the system, as in MIMO systems all parts of the system affect this metric. Both the antenna characteristics, MIMO signal processing algorithms implemented in the DUT or the propagation channel model where the DUT is being measured will have an effect on the ability of the DUT to properly demodulate the signal transmitted.

In this new field of research that opens on MIMO OTA characterization, arise three measurement methodologies (based on pre-existing techniques for SISO systems). Each of these three methods present a number of advantages and disadvantages, which has opened a long discussion in recent years trying to figure out which of them should be in the standard documents.

Table 5 presents a short summary (extracted from [8]), which compares some of the most important features of each of the three options.

TABle 5. Characteristic comparison between the three proposed OtA MEthodologies. SOURCE: [8]

\begin{tabular}{|c|c|c|c|}
\hline Attribute & MSRC Based & Anechoic Chamber Based & 2 stage method \\
\hline Major components & Reverberation chamber & $\begin{array}{c}\text { Channel emulator, probe } \\
\text { antennas, anechoic } \\
\text { chamber }\end{array}$ & $\begin{array}{c}\text { Channel emulator, probe } \\
\text { antenna, anechoic } \\
\text { chamber }\end{array}$ \\
\hline Number of probe antennas & $2-4$ & 8-32 (varies) & 1 \\
\hline $2 D / 3 D$ & $3 D$ & $2 \mathrm{D} / 3 \mathrm{D}$ (varies) & $2 \mathrm{D} / 3 \mathrm{D}$ \\
\hline Number of spatial clusters & 1 & 1-24 (varies) & $1-24$ \\
\hline $\begin{array}{c}\text { Power angular spectrum per } \\
\text { cluster }\end{array}$ & Uniform & Controllable & Controllable \\
\hline AS & Random & Controllable & Controllable \\
\hline PDP & Exponential decay & Controllable & Controllable \\
\hline DS & $\begin{array}{c}\text { Slightly controllable } \\
\text { decay }\end{array}$ & Controllable & Controllable \\
\hline Doppler shift & Limited & Controllable & Controllable \\
\hline Supported channel models & Uniform & $\begin{array}{l}\text { SCME, Single Cluster, } \\
\text { Uniform, Arbitrary }\end{array}$ & $\begin{array}{l}\text { SCME, Single cluster, } \\
\text { uniform, arbitrary }\end{array}$ \\
\hline Cross Polarization Ratio (XPR) & Constant & Controllable & Controllable \\
\hline $\begin{array}{l}\text { Ability to control interference } \\
\text { direction }\end{array}$ & No & Yes & Yes \\
\hline DUT size constraints & None & $0.5-4$ lambda (varies) & None \\
\hline Calibration equipment/method & 2-Port VNA & $\begin{array}{l}\text { Joint OTA link calibration } \\
\text { using 2-port VNA }\end{array}$ & $\begin{array}{l}\text { Chamber calibration by } \\
\text { 2-port VNA, second } \\
\text { stage calibration }\end{array}$ \\
\hline $\begin{array}{c}\text { Requires non-intrusive test } \\
\text { mode for antenna pattern } \\
\text { measurement }\end{array}$ & N/A & No & Yes \\
\hline $\begin{array}{c}\text { Throughput measurement } \\
\text { method }\end{array}$ & OTA & OTA & $\begin{array}{c}\text { Conducted via } \\
\text { temporary antenna } \\
\text { connector }\end{array}$ \\
\hline
\end{tabular}




\section{The Mode-Stirred Chamber: Working Principles and Latest Advances}

\subsection{Overview}

In Chapter 2 it has been shown a short overview of the main features of a MSRC, along with the other proposed techniques. This chapter presents a more in-depth study on the functioning mechanisms of an MSRC, as well as the evaluation of the current state of the art as far as emulation of non-isotropic channel models is concerned.

A MSRC is a highly conductive enclosed cavity typically equipped with metallic paddles and turntables. The independent movement of paddles and turntables dynamically changes the electromagnetic field boundary conditions. In this way the natural multimode electromagnetic environment inside the single cavity is stirred. With this continuous mode stirring in time, the chamber provides the same statistical distribution of fields independent of location, except for those observation points in close proximity to walls and nearby objects.

This required field uniformity also implies polarization balance in the chamber. At any observation point within the chamber, the field will vary from a maximum to a minimum as the different elements (stirrers and turntables) change the boundary conditions [14]. The standard deviation of the mean field throughout the chamber is typically the figure of merit used to assess the performance of the MSRC. In a perfectly-stirred MSRC, the real and imaginary parts of the rectangular components of the electric and magnetic field throughout the chamber are Gaussian distributed, independent with identical variances. Thus, the electric or magnetic field inside a perfectly stirred MSRC follows a single-cluster Rayleigh probability density function in 
amplitude and uniform distribution of phase, which resembles the multipath fading in urban scenarios of wireless communications systems.

These features make the MSRC an ideal solution for antenna characterization. In the last few years a sort of studies has been released extending the measurement capabilities of MSRCs. Parameters like diversity gain (DG) [17], antenna efficiency [18] [19] or MIMO capacity [20] [21] have been successfully measured using MSRCs. Different propagation channels has been emulated as well, such as Rayleigh [22] and Rician [23]. Even, antenna radiation pattern (which has been typically measured only in anechoic environments) can be measured now with MSRC's applying a time-reversal technique [24]. Also the influence of the user over the antennas, as well as the influence of the antennas over the user, has been studied using MSRC's [21] [25].

If we assume that the introduction of a matched antenna does not perturb the preexisting field distribution within the chamber, the power received by this matched antenna inside the MSRC is independent of the antenna gain, directivity, or equivalent area [15]. This, along with the repeatability and reliability of the stochastic reference fields emulated in the MSRC, makes them ideal candidates to evaluate antenna radiated power for wireless communications systems. Since for handheld wireless communications systems antenna radiated power-related parameters such as TRP and TIS are the standardized figures of merit, MSRCs have become a popular tool for evaluating wireless communication performance [26] [27].

Yet, propagating scenarios experienced by users outdoors rarely follow the behaviour of a uniform Rayleigh fading scenario with single-cluster isotropic scattering. A single-cluster assumes that waves that are reflected or diffracted at the receiver and propagated toward the receiver are grouped into just one collection, corresponding to a group of buildings or objects in a room. In urban environments, for instance, one can find several buildings on both sides of the street and each of them can be modelled as a cluster of scatters. Hence, to describe properly this scattering environment, multiple clusters are needed. An isotropic scattering scenario, also known as uniform, assumes that all angles of arrival at the receiver have equal probability, that is, there is no preferred direction of upcoming waves. A distribution of scatters that leads to a uniform distribution of angles of arrival is also difficult to justify in practice [28].

In consequence, recent years have witnessed a relatively large number of papers describing novel concepts using more general MSRCs with both hardware and software modifications to that of simple single-cavity MSRCs in order to overcome their innate limitations. With those modifications, the fields do not necessarily have to be constrained to a single cavity or even be provided in a reverberating mode to the researcher. In consequence, MSRCs may contain more than one metal cavity that 
could be coupled through a variety of means, including waveguides, slots or metal plates, among others. Likewise, the shape of these cavities does not have to be restricted to the canonical ones, and additional software control and algorithms allow extraordinary advantages to the researcher over conventional single-cavity MSRCs.

\subsection{Electromagnetic Analysis of a MSRC}

Assuming a rectangular cavity of dimensions $a \times b \times h$, it is possible to express all the propagated modes as either transverse electric (TE) or transverse magnetic (TM) to any of the three coordinate axis. Therefore, it is usual notation to write all the modes as transverse to the $z$ direction. Then, the electric fields $E_{I m n}$ of the eigenmode defined by the index $I, m$ and $n$, propagating inside the metallic cavity can be written as follows [29]

$$
\begin{gathered}
E_{l m n}^{T E}(r)=-\hat{x} k_{y} \emptyset_{l m n}^{x}(r)+\hat{y} k_{x} \emptyset_{l m n}^{y}(r) \\
E_{l m n}^{T M}(r)=-\hat{x} k_{x} k_{y} \emptyset_{l m n}^{x}(r)-\hat{y} k_{y} k_{z} \emptyset_{l m n}^{y}(r)+\hat{x}\left(k_{x}{ }^{2}+k_{y}{ }^{2}\right) \emptyset_{l m n}^{z}(r)
\end{gathered}
$$

Where

$$
\begin{gathered}
\emptyset_{l m n}^{x}(r)=\frac{\varepsilon_{l m n}}{\sqrt{a b c}} \cos \left(k_{x} x\right) \sin \left(k_{y} y\right) \cos \left(k_{z} z\right) \\
\emptyset_{l m n}^{y}(r)=\frac{\varepsilon_{l m n}}{\sqrt{a b c}} \sin \left(k_{x} x\right) \cos \left(k_{y} y\right) \sin \left(k_{z} z\right) \\
\emptyset_{l m n}^{z}(r)=\frac{\varepsilon_{l m n}}{\sqrt{a b c}} \sin \left(k_{x} x\right) \sin \left(k_{y} y\right) \cos \left(k_{z} z\right) \\
k_{x}=\frac{l \pi}{a}, \quad k_{y}=\frac{m \pi}{b}, \quad k_{z}=\frac{n \pi}{h} \\
\varepsilon_{l m n}=\left\{\begin{array}{cc}
2 & \text { if } l, m \text { or } n=0 \\
\sqrt{8} & \text { if } l, m \text { and } n \neq 0
\end{array}\right.
\end{gathered}
$$

And the cut-off frequency for a given mode can be expressed as,

$$
k_{l m n}^{2}=\left(\frac{2 \pi f_{0, l m n}}{c}\right)^{2}=\left(\frac{l \pi}{a}\right)^{2}+\left(\frac{m \pi}{b}\right)^{2}+\left(\frac{n \pi}{h}\right)^{2}
$$

And therefore,

$$
f_{0, l m n}=\frac{c}{2 \pi} \sqrt{\left(\frac{l \pi}{a}\right)^{2}+\left(\frac{m \pi}{b}\right)^{2}+\left(\frac{n \pi}{h}\right)^{2}}
$$

An important parameter to characterize a MSRC is the number of modes propagating for a given frequency. In order to properly count the number of modes that are actually propagating inside the cavity, it is necessary to take in account the degenerated modes (with the same cut-off frequency). When none of the indexes is equal to zero, then both TE and TM modes are propagating. On the contrary when any 
of the indexes are equal to zero, just one mode (TE or TM, depending on the index) will propagate. Therefore, it is possible, to actually count the exact number of modes propagating in a given cavity for a given frequency.

However, it is simpler to use some numerical approximations to perform that estimation [29],

$$
N_{\text {modes }}(f)=\frac{8 \pi}{3} a b c \frac{f^{3}}{c_{0}^{3}}-(a+b+h) \frac{f}{c_{0}}+\frac{1}{2}
$$

Where $c_{0}$ is the speed of light in the air (which is assumed to be the propagation medium). This expression is valid for rectangular MSRC, and other expressions can be found for different geometries.

The number of modes defines the frequency range where the MSRC has a certain level of uniformity. 100 modes is commonly taken as a reference value to guarantee a good performance. In [14], it is defined the minimum usage frequency of a MSRC as three times the frequency of the first propagated mode.

All the formulation we have seen provides a discrete spectrum of modes propagating in a MSRC. This is true in the case where the metallic walls of the cavity have no losses. However, there is always a certain amount of losses in the metallic cavity, and that allows to exist a certain modal bandwidth $B W_{Q}$, making continuous the discrete spectrum of modes [30]. Therefore, a given mode with eigenvalues $I, m, n$ will propagate with a finite bandwidth that can be expressed as [14],

$$
f_{l m n}=f_{0, l m n} \pm B W_{Q}
$$

Where

$$
B W_{Q}=\frac{f}{Q}
$$

Where $Q$ is the so called "quality Factor"or $Q$-factor of a MSRC. The $Q$-factor of a cavity, describes the ability of the cavity to store energy. This ability is mainly influenced by the losses in the cavity, where the dominant component of losses is in the walls.

Therefore, the $Q$-factor depends mainly on three factors: dimensions of the cavity, losses and frequency, and can be expressed as [14],

$$
Q=\frac{16 \pi^{2} V}{\eta_{t x} \eta_{r x} \lambda^{3}} \frac{P_{r x}}{P_{t x}}
$$

Where $V$ is the volume of the cavity, $\eta_{t x}$ and $\eta_{r x}$ are respectively the efficiencies of the trasmitting and receiving antennas, $\lambda$ is the wavelength, and the relation $P_{r x} / P_{t x}$ quantifies the losses in the cavity. 
Summarizing, it has been presented in this section a short overview of the main parameters that define the behavior of a MSRC. Number of modes, $Q$-factor or minimum frequency of use can be considered as the main parameters that distinguish a good MSRC.

\subsection{OTA Measurement Procedure Using a MSRC: The Rayleigh Distributed Environment}

\subsubsection{General Procedure}

An MSRC contains a set of mode stirrers that changes the boundary conditions of the main cavity of the chamber. The metal cavity, along with the stirrers, provides a multi-reflective environment which is repeatable and can be statistically studied. The repeatability and statistical nature of MSRCs make them a versatile tool for measuring a wide variety of MIMO antenna parameters [15] [23] [28] [31] [32]. In its conventional design, an MSRC uses a single cavity. With perfect stirring the electric or magnetic field inside the single cavity follows a Rayleigh PDF in amplitude. This initial design has been densely studied in the literature [20] [33] [34]. Then, the scattering parameters of a system can be analyzed statistically, by averaging over a stirring sequence. As it has been shown in previous sections, the number of stirring positions required depends on the frequency, the stirring efficiency of the mode stirrers and the size and shape of the chamber.

But to obtain these parameters, it is required the use of a vector network analyzer (VNA) to determine the scattering parameters (S-parameters) of the different transmitters and receivers ports, for each possible position of the stirring sequence. The measurement procedure should be prepared for each antenna port with all the other ports terminated in $50 \Omega$, and repeating the procedure with the reference antenna so the field is the same when measuring each antenna port.

Then, the measured $\mathrm{S}$ parameters between the port $i$ to the port $j$ can be averaged over the whole stirring sequence as follows,

$$
\overline{S_{l \jmath}}=\frac{1}{N} \sum_{k=1}^{K} S_{i j, k}
$$

Where $K$ is the total number of stirring positions.

The result of the measured parameters represent an estimate of the channel matrix $H$ of the communication channel between the transmitting antennas mounted on the MSRC walls and the MIMO receiver antennas being evaluated. The coefficients of the channel can be represented as, 


$$
h_{i j}=\frac{s_{i j} \sqrt{e_{r e f}\left(1-\left|\overline{s_{l j}}\right|^{2}\right)}}{T_{r e f}}
$$

Where $T_{\text {ref }}$ is the channel transfer function for the reference antenna, and $e_{\text {ref }}$ its efficiency (which must be known). Then the estimation of the MIMO channel matrix with $N$ transmitting and $M$ receiving antennas, can be expressed as,

$$
H_{N x M}=\left[\begin{array}{ccc}
h_{11} & \cdots & h_{1 M} \\
\vdots & \ddots & \vdots \\
h_{N 1} & \cdots & h_{N M}
\end{array}\right]
$$

In the following sections it will be shown how to measure some important FoMs for antennas and wireless systems characterization, using a MSRC.

\subsubsection{Antenna Correlation}

Antenna correlation is the figure-of-merit which has been commonly accepted to be a good indicator of the MIMO performance of an antenna pair. As showed in [35], correlation affects MIMO capacity, which is clearly decreased when antennas at the receiver are highly correlated.

The complex correlation coefficient of two antennas can be calculated from the complex transmission coefficients $\left(S_{21}\right)$ between the exciting antenna of the MSRC and the antenna pair under test, by [36]

$$
\rho=\left|\rho_{\text {complex }}\right|=\left|\frac{\sum_{k=1}^{N}\left(S_{21,1}(k)-\overline{S_{21,1}}\right)\left(S_{21,2}(k)-\overline{S_{21,2}}\right)^{*}}{\sqrt{\sum_{k=1}^{N}\left|S_{21,1}(k)-\overline{S_{21,1}}\right|^{2} \sum_{k=1}^{N}\left|S_{21,2}(k)-\overline{S_{21,2}}\right|^{2}}}\right|
$$

where $k$ stands for the current stirrer position, and

$$
\overline{S_{21}}=\frac{1}{K} \sum_{k=1}^{K} S_{21, k}
$$

- $\quad K$ is the total number of stirrer positions over a whole sequence of stirring movement.

- $\quad S_{21,1}$ is the $S_{21}$ parameter between the transmission antenna and the reception antenna number one.

- $\quad S_{21,2}$ is the $S_{21}$ parameter between the transmission antenna and the reception antenna number two.

\subsubsection{DG}

When a radio wave is observed in a steady-state over the time, it is observed that there exists a fluctuation in the signal amplitude that can even break a wireless connection. That effect is known as fading, and it is one of the most important effects 
in a radio propagation characterization. However, it is possible to mitigate that effect by using more than one antenna at the receiving part of the link. This can be achieved if the signals in each of the receiving antennas are decorrelated enough. In order to introduce decorrelation it is possible to use several techniques, where the most important are; spatial separation between antennas, the use of different polarizations or introducing differences in the radiation pattern.

DG is a metric which quantifies the improvement created by the existence of more than one antenna over a reference case. The reference case can be either a reference antenna or one of the antennas in the system (best case is used usually), or even the average of all antennas.

Four different schemes are mainly used to combine the signals coming from the different receiving antennas (branches) [37],

- $\quad$ Scanning diversity: this technique consist on switching between antennas, in such a way that an antenna is selected as receiver until it drops below a certain level, and then a selector scans the rest of antennas in a fixed sequence to find a signal above the threshold level. This technique is simple and usually used in systems with two antennas feeding a single receptor. However, in complex systems it is not used, due to it relatively bad performance compared to other methods.

- $\quad$ Selection diversity: also referred as switch diversity, this technique is also a switching technique. However, in this technique, the selector is continuously checking the receiving signals, in such a way that the receiver has the best signal of all antennas at every moment. This technique can be mathematically expressed as,

$$
x= \begin{cases}1, & \text { for } j=k \\ 0, & \text { for } j \neq k\end{cases}
$$

Where $a_{j}(t)$ is the output signal branch of the switching system, and $k$ denotes the index of the branch with the maximum power at a given moment $t$.

- Maximum ratio combining (MRC): this technique uses all the signals from all the receiving antennas, in such a way that the different branches $\left(p_{i}\right)$ are weighted proportionally to the conjugate of the signal voltage and the inverse of the branch noise power. Let's call $x_{k}$ the incoming signal for the $k_{t h}$ antenna in a system with $P$ antennas, therefore it can be written, 


$$
x=\sum_{k=1}^{P} \sqrt{\frac{x_{k}^{2}}{\overline{n_{k}^{2}}}}
$$

Where $n_{k}^{2}$ denotes the additive white Gaussian noise present for each signal.

- Equal-Gain Diversity: also referred as selection combining (SC), consist of a particular case of the MRC case where the gain of all the signals is assumed equal. Therefore, it can be written,

$$
x=\sum_{k=1}^{P} x_{k}
$$

DG is therefore the quantification of the improvement created by the diversity antennas. It can be expressed as,

$$
D G=\frac{P_{\text {div }}}{P_{\text {ref }}}
$$

Being $P_{\text {div }}$ the power of the compound signal (after the diversity scheme implementation), and $P_{\text {ref }}$ is the power of the reference branch. Both power levels must be read it from a cumulative density function (CDF) representing the probability that an arbitrary power sample is smaller than a certain power level. In Figure 7 it is shown an example of a DG measurement.

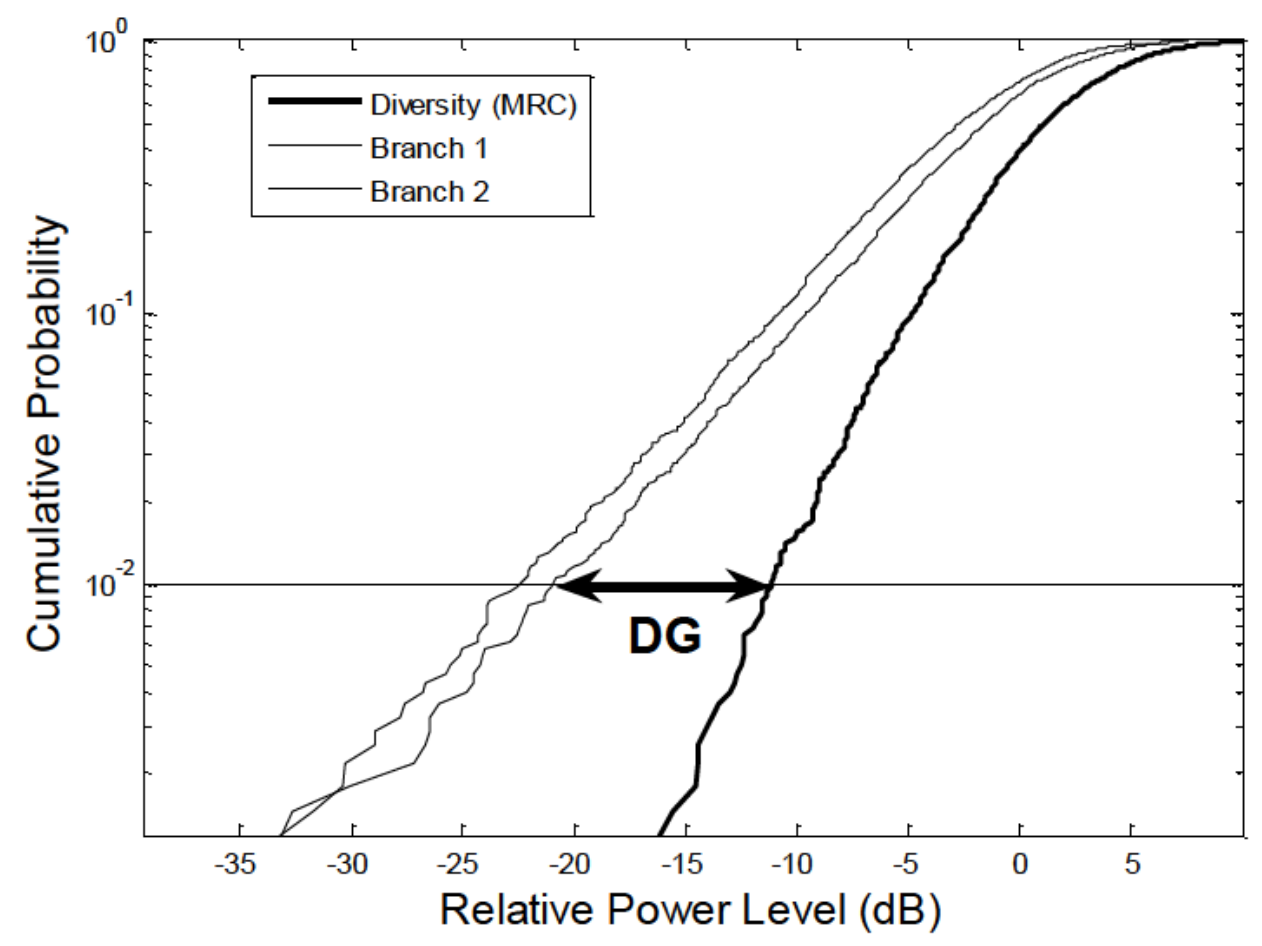

Figure 7. DG of a given dual-antenna for calculated at the $99 \%$ signal level reliability (only $1 \%$ of power levels below the power values used for DG estimation). 
There are several definitions of DG, which vary depending on which case is used as the reference case, since DG is the improvement obtained by using multiple antennas instead of using the reference antenna. In the case of apparent diversity gain (ADG) the reference is the branch or antenna that provides a stronger signal, while for the effective diversity gain (EDG) it is a reference calibrated antenna [38].

\subsubsection{MIMO Capacity}

The capacity of a system is defined as the maximum possible speed transmission, such that the error probability is arbitrarily small. As it is showed in [39], the capacity of a system with $N$ transmitting and $M$ receiving antennas, assuming that the channel state is only known at the receiver can be expressed as a function of the channel matrix $H$,

$$
C=\log _{2} \operatorname{det}\left(I_{m}+\frac{S N R}{N} H H^{H}\right) \quad[\mathrm{bits} / \mathrm{s} / \mathrm{Hz}]
$$

Where $I_{m}$ is the $N \times M$ identity matrix, SNR stands for the signal-to-noise ratio present at the channel, $N$ is the number of transmitting antennas and $H^{H}$ is the Hermitian transpose matrix of $H$.

The formula presented in (25) is valid for the instantaneous capacity, and therefore it must be averaged over a whole stirring sequence when it is measured in a MSRC,

$$
C=\sum_{k=1}^{K} \log _{2} \operatorname{det}\left(I_{m}+\frac{S N R}{N}\left(H H^{H}\right)_{k}\right) \quad[\mathrm{bits} / \mathrm{s} / \mathrm{Hz}]
$$

It is worth mentioning, that for an independent and identically Rayleigh distributed environment this capacity can be approximated to $C \approx \min (N, M) \log _{2}(\mathrm{SNR})[\mathrm{bits} / \mathrm{s} / \mathrm{Hz}$ ] for high values SNR. That indicates a theoretical great potential for growth in the number of antennas used, since for every three $d B$ increase in SNR the improvement leads to an increase in capacity of $\min (N, M)[b i t s / s / H z]$ [7]. This theoretical improvement is in practice much lower, due to some effects found in real situations that away from reality the assumption of an independent and identically Rayleigh distributed environment.

\subsubsection{Rician $k$-factor}

It has been showed before that the natural environment generated in a MSRC is a Rayleigh distributed environment, where both the real and imaginary parts of the signal follows a Gaussian distribution. This distribution resembles the distribution typically found in multipath environments with Non Line-of-Sight (NLoS = no direct vision between the transmitter and the receiver). 
However, that is a particular case of a more general signal distribution that can be found in real environments, that is called Rician distribution [40], which is the distribution found when there is Line-of-Sight ( $L O S=$ direct vision between the transmitter and the receiver). A Rician distribution is defined by a parameter called $k$ factor, which is defined as the ratio between the LoS component and the NLoS components.

According to [23] it is possible to calculate the Rician $k$-factor inside a MSRC from the measured transmission scattering parameters, using the following equation,

$$
k=\frac{d_{R}^{2}}{2 \sigma_{R}^{2}}=\frac{\left(\left|\overline{S_{21}}\right|\right)^{2}}{\overline{\left|S_{21}-\overline{S_{21}}\right|^{2}}}
$$

Where $\sigma_{r}$ is the radius of the clutter of data (as seen in Figure 8) and quantifies the dispersion (multipath components) of the data. On the contrary, $d_{R}$ is the distance of the centroid of the clutter from the origin and reflects the in-phase amplitude offset introduced by the direct component.

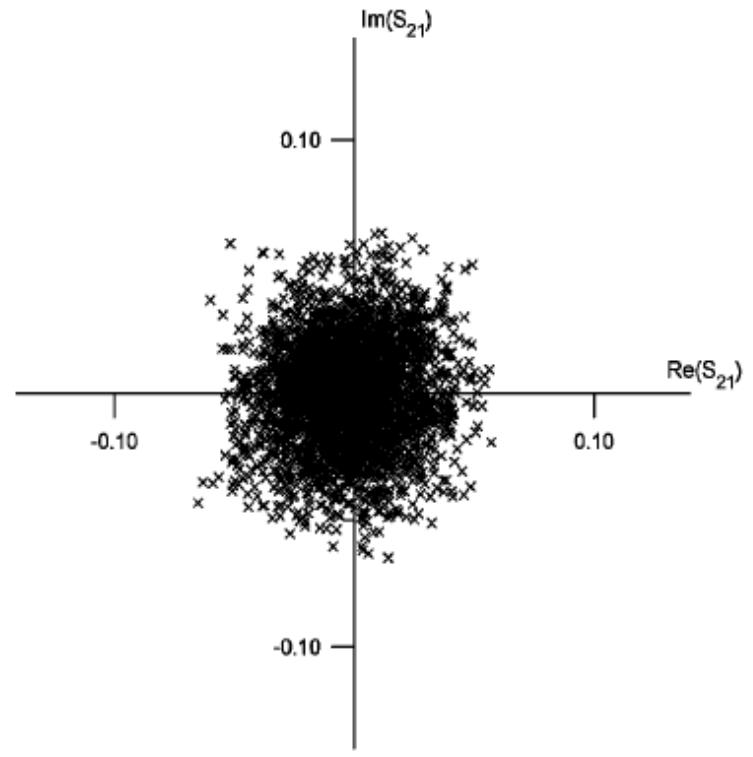

a)

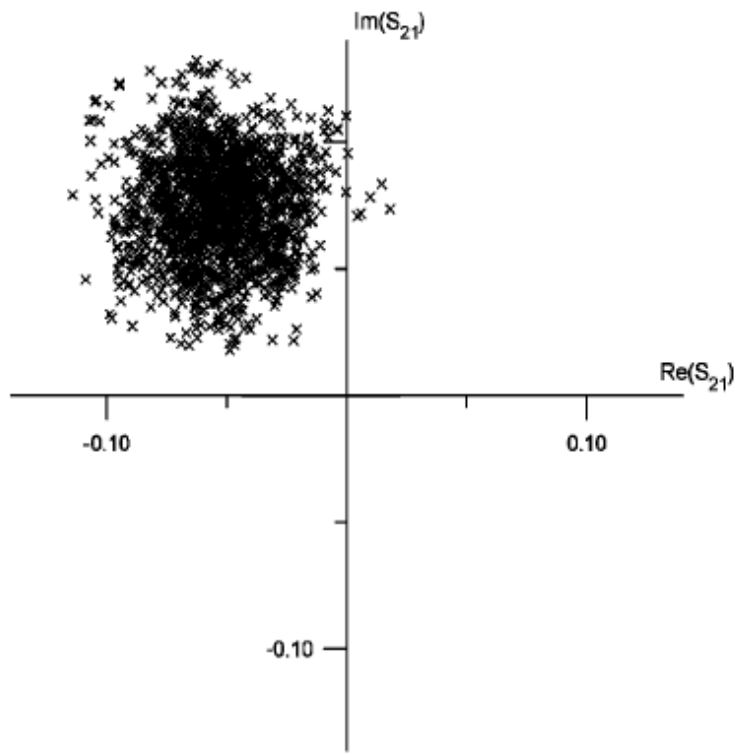

b)

Figure 8. Comparison between the scatter plots of a Rayleigh distributed $S_{21}$ measurement (left) and a Rician distributed (right). SOURCE: [23]

\subsubsection{Data Throughput}

All FoMs studied so far are aimed to characterize the passive part of a wireless device, ie. the antenna module. However, it becomes important to characterize the whole system, including both antennas, radio frequency amplifiers and electronics in charge of the signal processing. For this analysis, as seen in Chapter 2, FoMs were proposed as the TRP and TIS for single antenna systems. However, in the case of multi- 
antenna systems the most widely used FoM is the end-to-end throughput, which quantifies the data stream (in bps) transmitted effectively throughout the whole link (including transmitter, medium and receiver).

As it was defined in Eqs. (1) and (2), throughput is calculated from the BLER measured value, and it stands for the time-averaged number of correctly received transport blocks by the DUT. Throughput by itself just quantifies the amount of bits correctly transmitted through the whole communication system, for a given state of the system. However it is common to present throughput as a function of received power, so it is possible to appreciate the degradation (in bps) created by the reduction in power. An example is shown in Figure 9, where two throughput curves are presented (same device under two different channel models).

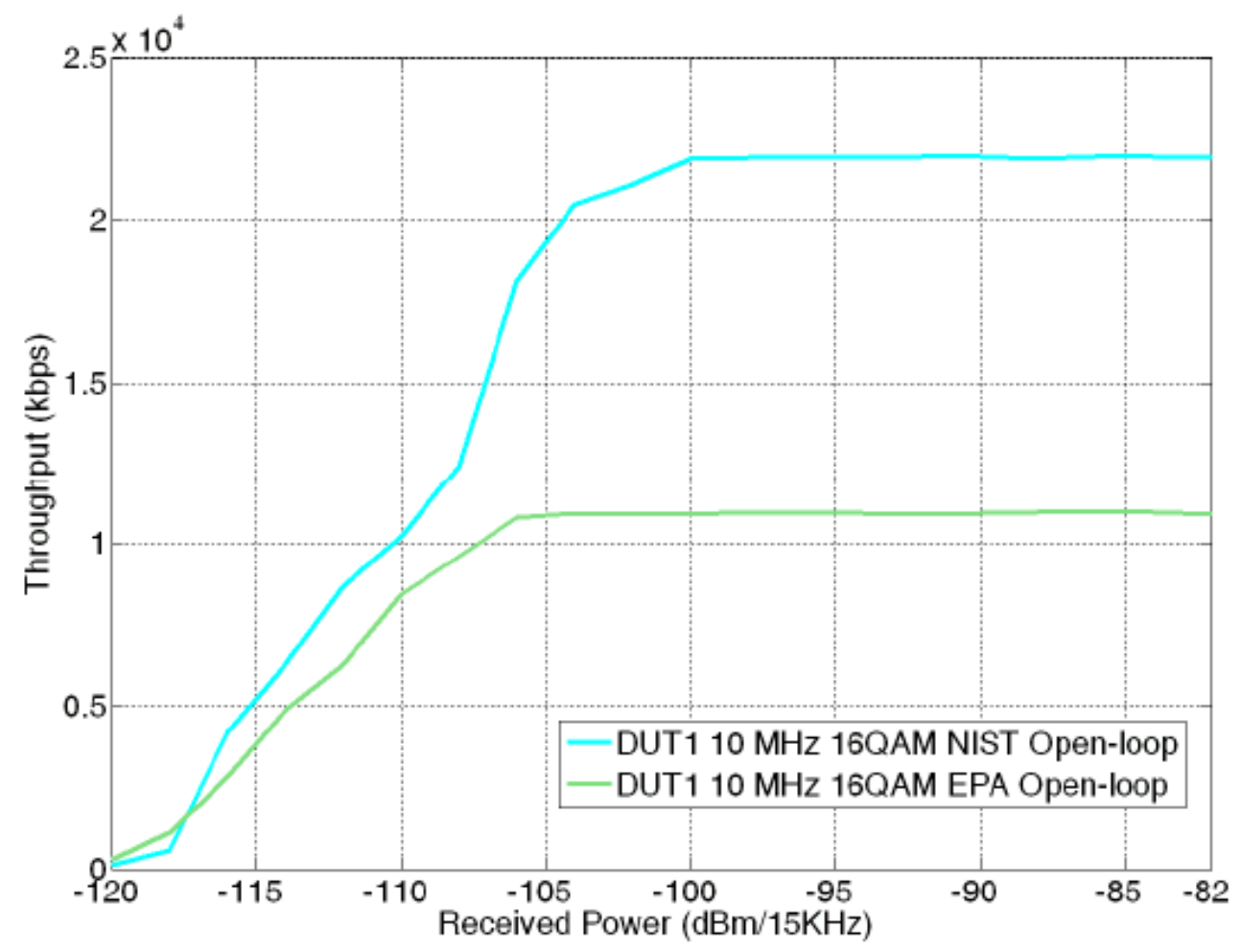

Figure 9. Data throughput plotted as a function of the received power for a LTE device. SOURCE: [41]

\subsection{Latest Advances In Non-Isotropic Channel Emulation using MSRCs}

\subsubsection{Hardware-Based MSRCs Advances}

\subsubsection{Rician Fading Emulation Using Directive Antennas inside the MSRC}

One important enhancement on the MSRC field is related to the ability to emulate Rician-fading environments. The Rayleigh-fading case $(k=0)$ typically emulated by an MSRC is a special case of a more general Rician-fading case $(k>0)$. The Rician $k$-factor 
is defined as the ratio between the power of the coherent component (corresponding to the direct path) over the power of the incoherent component (corresponding to the scattered component) of the received field. In fact, when the MSRC is not perfectly stirred, the unstirred field component being preserved defines a Rice field in coexistence with the Rayleigh field generated by the stirred components. Stochastic plane wave superposition and separation theories can be employed to obtain both stirred (equivalent to NLOS or Rayleigh-fading components) and unstirred contributions (equivalent to LoS or Rician-fading components).

Yet, in most cases the separation of these two components is aided by employing an excitation source that is pointed toward the DUT, and then it is assumed that all wall reflections interact with the paddles [23]. With only one transmitting antenna, other ways of controlling the $k$-factor are now possible in an MSRC. This includes that the transmitting antenna, with a well-defined radiation patter (azimuth change), can be rotated with respect to the DUT, altering the distance between the transmitting antenna and the DUT (distance change), changing the polarization orientation of the transmitting antenna (polarization change), or varying the cavity's $Q$-factor by chamber loading ( $Q$-factor change) [23].

Some variable $k$-factor results in [23] are illustrated in Fig. 10. If two transmitting antennas are used, a wide range of $k$-factors can be obtained by pointing one of them toward the DUT and the other one toward the stirrers [23]. Interestingly, the $k$-factor obtained in a mode-stirred chamber has also been found to be dependent on the number and position of absorbers placed within the main cavity [42].

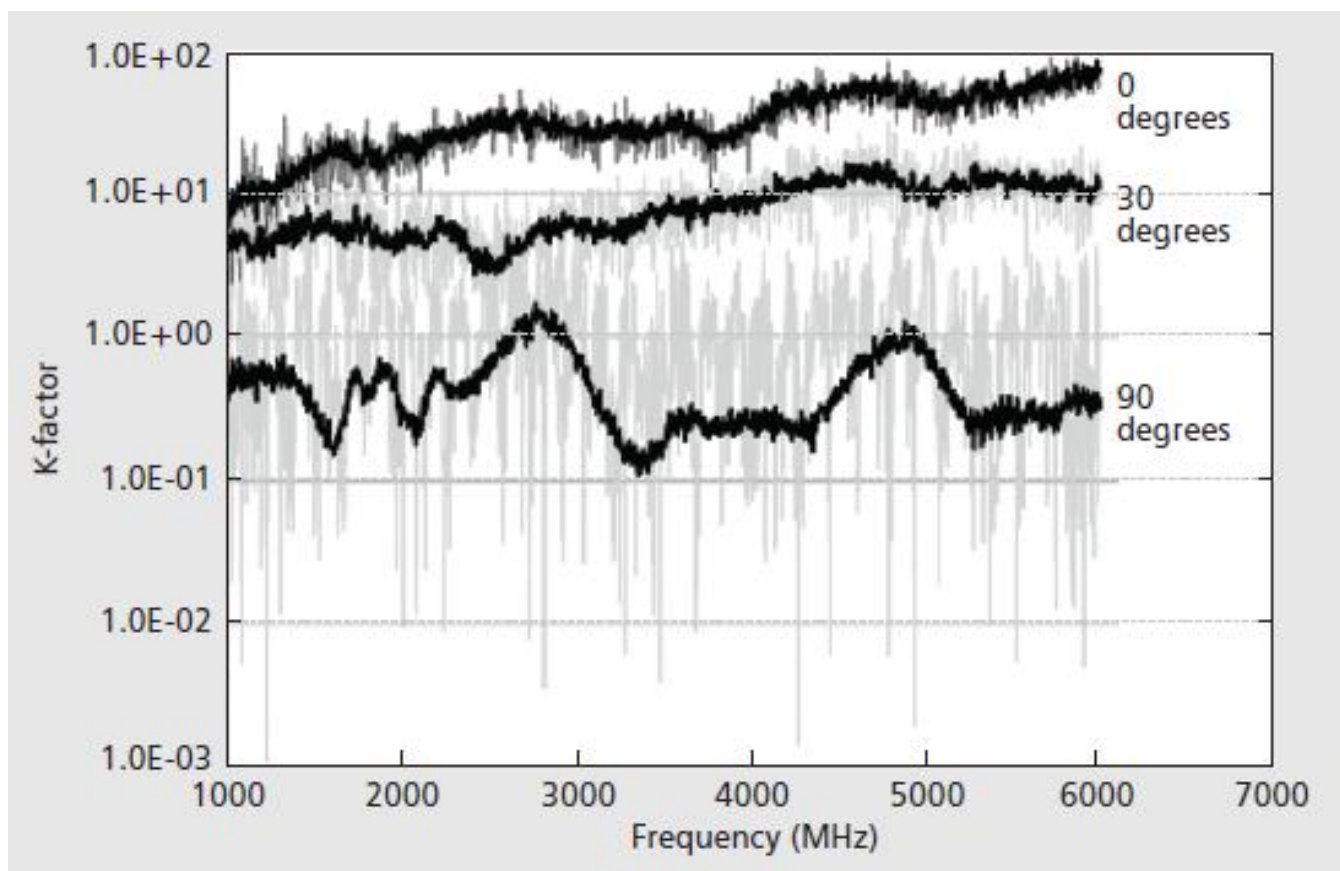

Figure 10. Variable $k$-factor in a mode-stirred chamber when altering the azimuth orientation of the transmitting antenna. SOURCE: [23]. 


\subsubsection{Hyper-Rayleigh Fading Emulation}

While Rayleigh and Rician fading are commonly used in wireless propagation emulation, small scale fading encountered in several new scenarios such as vehicle-tovehicle systems present frequency-dependent and spatially-dependent fading whose severity exceeds that predicted by the Rayleigh fading model. These scenarios are coined as Hyper-Rayleigh, and a very recent paper has been able to accurately emulate these scenarios using a modified MSRC [43]. In [43], an electrically switched multielement antenna array was added to an MSRC, and the enclosure size was made considerably smaller than conventional MSRC for the same tested frequency range. Figure 11 depicts the plots of signals experiencing Rayleigh, Rician, and Hyper-Rayleigh fading scenarios in the MSRC of [43].

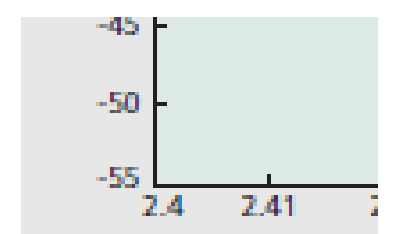

Figure 11. Example of Hyper-Rayleigh fading, compared to Rayleigh and Rician fading. SOURCE: [43].

\subsubsection{Non-Canonical Configurations}

By carefully controlling the excitation source of an MSRC, the homogeneity and isotropic characteristics of the field at a specific position can be controlled. The key to obtain enhanced performance is the ability to shift and weight each mode within the chamber, and an array of exciting antennas was proposed to alleviate the mechanical requirements of MSRCs [44]. This is straightforwardly derived if one takes into account the fact that the field strength at any observation point within the chamber can be obtained by the integration in the source.

Changing the sources therefore changes the resulting field strengths. This particularly useful advance has even made researchers coin new terms for MSRCs, such as scatter-field chamber or source-stirred chamber, among others. In order to excite additional transversal electromagnetic modes, other non-canonical chamber configurations have been proposed. By exciting the chamber with transmission lines 
[45], for example, new transverse electric magnetic (TEM) modes that are transversal to those wires can be excited, further increasing the frequency range of operation. In particular, for the same cavity size, the lowest usable frequency becomes smaller. Different wire and phase shift excitations are also possible. Other noncanonical configurations include those contributions that employ a variable geometry, a moving wall [46], or non-parallel walls [47]. In such non-canonical MSRCs no eigenmodes exist and a diffuse, statistically uniform field is created without the use of a mechanical mode stirrer. As a result, test times can be drastically reduced.

One recent contribution for enhanced emulation using MSRCs is the opening of the door [48]. The aperture of the door transforms one wall that was perfectly electric into a perfectly magnetic wall, but at the same time with a varying aperture degree. Some modes will try to propagate through the opening, and therefore the chamber can no longer be called a reverberation chamber as both reverberating and non-reverberating modes exist. In this way, non-isotropic fading emulation can also be performed using a mode-stirred chamber, providing for a different number of multipath components (MPC), AoA or AS of the emulated scenarios.

Furthermore, the opening of the door can be used for enhancing the accuracy of the chamber for performing antenna radiated power measurements by a more accurate characterization of losses in the chamber by this opening of an aperture [15]. With the available manipulation of diverse spatial fading multipath characteristics using MSRCs, another important step was the ability to control the time-dependent fading performance by being able to emulate variable root-mean square (RMS) DS. Effects such as Doppler spread and fading, which are a consequence of a dynamically moving environment, can also be emulated inside an MSRC by moving the paddles with different speeds or using them in stepped or non-linear modes. With the use of absorbers in [49], different RMS DS profiles can also be achieved. The ensemble average of the magnitude squared of the impulse response of the MSRC is referred to as the PDP and it is the way to include effects due to time-varying multipath. The shape of the PDP can have adverse effects on the performance of digital communication systems. The RMS DS of the PDP is often used to characterize a wireless communication environment because it is directly related to the BER performance of a channel.

The BER is an end-to-end performance measurement that quantifies the reliability of the entire radio system from bits in to bits out. Standardized channel models are typically characterized by RMS DS. As the RMS delay spread in an MSRC has been found to be proportional to the chamber $Q$-factor for a given frequency, this is yet another sign that very accurate standardized channel fading emulation is possible with 
MSRCs. This includes emulating the behaviour of the BER for different stirrer velocities [50] [51] and chamber loadings [49] [51], as illustrated in Fig. 12 [49].
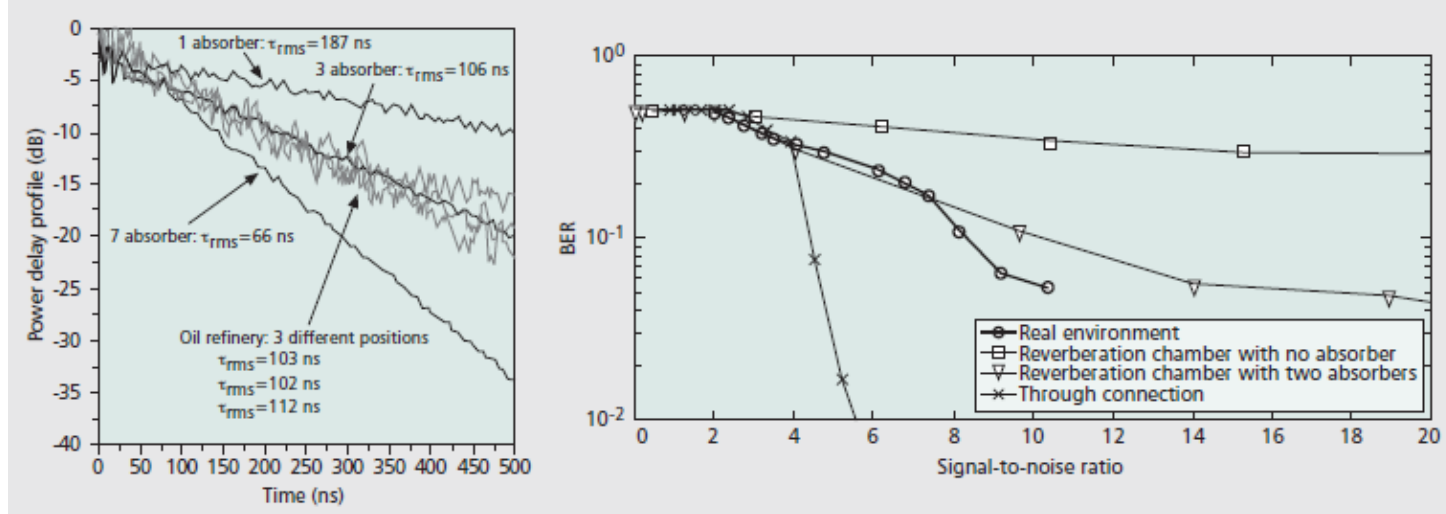

Figure 12. Different PDPs (left) and BER (right) measured using an MSRC. SOURCE: [49].

\subsubsection{MSRC's With Multiple Cavities}

Another important advance is the use of multiple cavities in order to provide for some control of a complex multipath environment consisting on diverse clusters with different fading characteristics. A possibility is to use a metal plate with different-size irises separating two cavities. This can give some control over which modes are coupled to the main cavity and also enlarge the delay spread at the main cavity in comparison to single-cavity MSRCs [48]. Another possibility is to connect two cavities with waveguides or wires [52], as illustrated in Figure 13. With this modification, the rank channel can be altered, and complex MIMO fading characteristics such keyholes can also be emulated. This enriches the emulating possibilities of MSRC, which now include the ability to emulate degenerated $H$ matrices as it happens in tunnels, for example.

With multiple cavities, not only the propagation characteristics of the transmitter and receiver can be modified independently, but MSRCs with multiple cavities can also reduce the typically high elevation angular spread of traditional single cavity MSRCs. Variable RMS DS have also been obtained with coupled cavities, which have demonstrated their ability to emulate indoor environments, wideband in-vehicle environments [53], or metallic windows, tree canopies, walls and other artefacts in buildings [54]. Interestingly enough, it has been found that for a typical metal-framed window structure, the MIMO capacity is greater than that without metal frames. For an $8 \times 8$ antenna system, the MIMO capacity is increased by about 2.5 times when metal frames are introduced, and the presence of leaves increases that capacity even more when the transmitted power is kept constant [54]. These enhancements have paved the way for new MSRC testbeds for MIMO systems able to emulate standardized fading channels. 


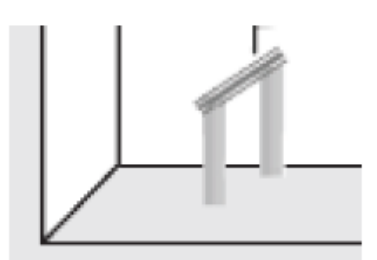

Figure 13. An MSRC with two coupled cavities. SOURCE: [52].

\subsubsection{Use of a Cascaded MIMO CE}

While specific power delay profiles can be replicated by adding certain amounts of absorbers and averaging over many different paddle positions, this configuration will always reflect the natural response of the MSRC (which is typically a single decaying exponential). However, in [55] the transmitter's excitation signal was injected into a fading emulator prior to introducing it into the chamber. In this way, it was created a channel response having multiple discrete clustered distributions, typically found in both urban and suburban settings where reflecting structures may be located far from the receiver. Very accurate emulation of these realistic environments can be performed using the method described in [55]. A clear advantage of this method compared to the one employed in the next section is the use of only one chamber. The disadvantage is clearly the requirement of a MIMO CE.

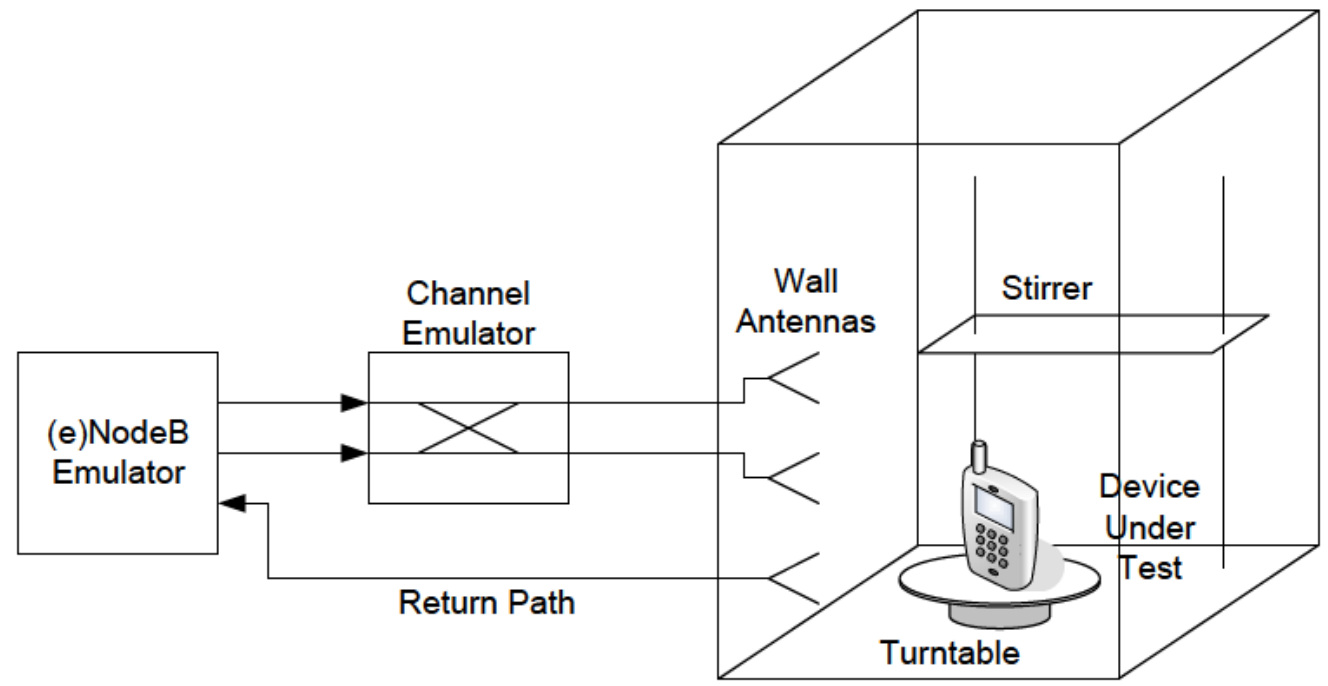

Figure 14. Test bench configuration for test using a MIMO CE cascaded with a MSRC for a 2x2 MIMO configuration SOURCE [8]:. 


\subsubsection{Software-Based MSRC's Advances}

\subsubsection{Time-Reversal Techniques}

Until this point, there have been presented a number of hardware modifications that extend the measurement capabilities of the MSRCs. However in [24] a different approach is proposed: make a software post-processing of the measured signal so that it is possible to get free space conditions, avoiding reflections produced in the walls of the cavity metal. This technique is defined as time-reversal, and is used in [24] to measure the antenna radiation pattern.

The proposed technique based on the field distribution within the MSRC generated when a DUT is excited with pulsed signal, behaves in a short period of time as if the propagation will be held in free space (as the signal has not yet interacted with the metallic walls). The proposed setup is shown in Fig. 15,

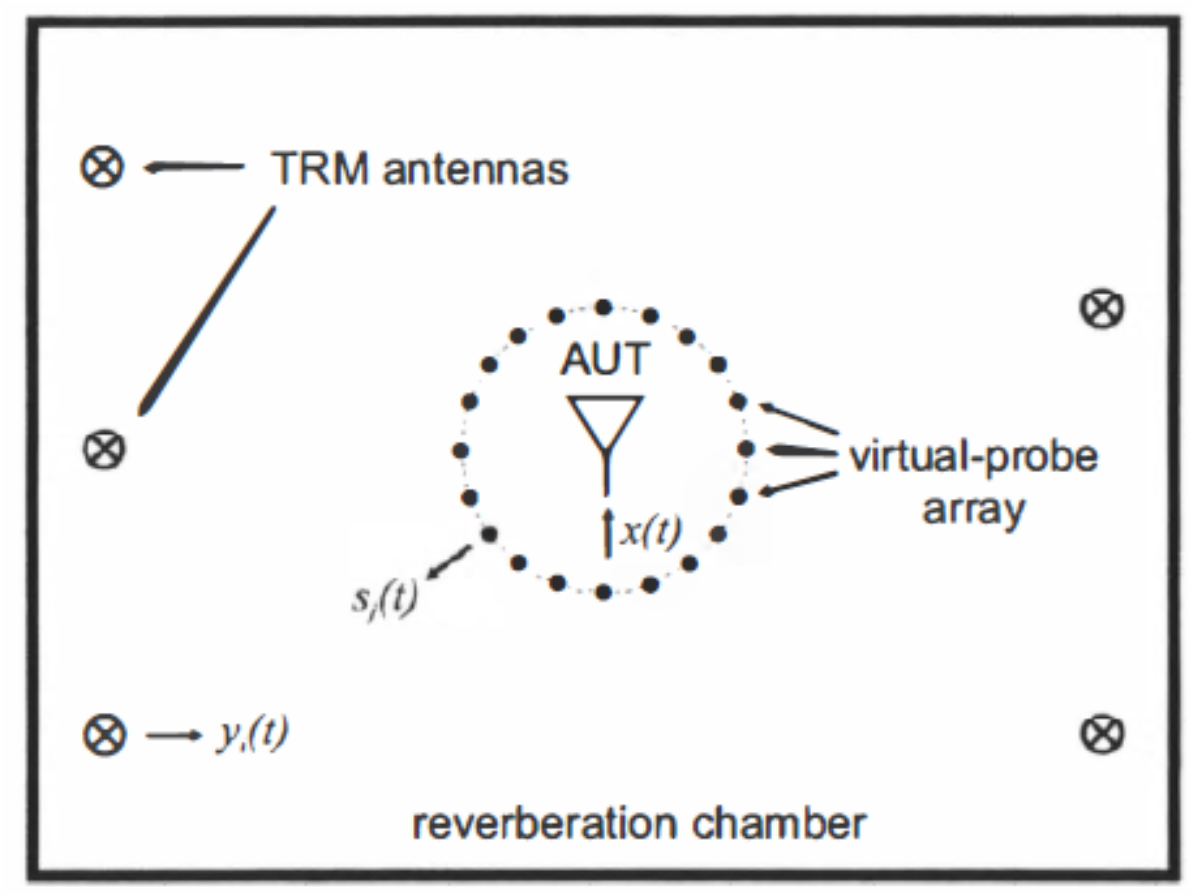

Figure 15. Setup for time-reversal measurements in a MSRC. SOURCE: [24].

The basic procedure consists in three steps:

- Virtual-probe array calibration: first of all it is necessary to calibrate the propagation environment in the MSRC. To do that, the channel matrix between the virtual-probe array (as shown in Figure 15) and the time-reversal mirror (TRM) antennas, must be carefully evaluated. Therefore, channel coefficients $h_{i j}$, between the $i_{t h}$ virtual-probe antenna and the $j_{t h}$ TRM antenna is known.

- $\quad$ DUT excitation with a pulsed signal and measurement with the TRM antennas: this part is actually the measurement part of the process. The virtual-probe 
array is extracted from the MSRC, and then the DUT is excited with a pulsed signal $(x(t))$ that satisfies the pulse time to be $T<d /\left(2 c_{0}\right)$, where $d$ is the shortest lateral dimension of the cavity, and $c_{o}$ is the speed of light in air, in order to actually emulate the free-space condition. The received signals $y(t)$ at the TRM antennas are then stored.

- $\quad$ Fast Fourier Transform (FFT) of the measured signals: finally, as the channel matrix is known, it is possible in the Fourier domain to calculate the signal that the virtual-probe array would have captured if it were present during the measurement (that is why is called virtual). Therefore, it is possible to express the virtual signals around the DUT as:

$$
S_{j}(f)=\sum_{i=1}^{M} Y_{i}^{*}(f) H_{i j}(f)
$$

Then, after reconversion to the time domain, $s(t)$ must be applied a rectangular or gating function to avoid any component outside the free space time window.

Summarizing, with this technique it has been shown the possibility of characterization of antennas in free space using MSRCs, in addition to the traditionally used anechoic chambers. FoMs as the radiation pattern can be measure accurately, just using a FFT-based post-processing algorithm applied to a measurement performed in a MSRC.

\subsection{Conclusions}

In the last few years, different advances have made MSRCs to solve the inherent limitations of conventional single-cavity MSRC for wireless communication performance evaluation. It is now clear that MSRCs have considerably improved the Clarke's model followed by conventional single-cavity MSRCs.

However, the proposed methods to extend the emulation capabilities of MSRC's from the naturally emulated Rayleigh distribution are still difficult to control since they require complex hardware modifications in the main cavity. Thus, it justifies the need to extend the emulation capabilities of the MSRC to complex environment through the use of software techniques, which will be studied in Chapter 7 of this thesis. 


\section{The MIMO Propagation Channel Model for Wireless Devices Testing}

\subsection{Overview}

\subsubsection{The MIMO Channel Model}

The MIMO propagation channel can be modelled from a theoretical point of view, seeing it as a linear filter, and therefore uniquely characterized by its impulse response $h(\tau)$, which is a function of delay. If both transmitter and receiver antennas, and the elements that cause multipath reflections are static, then the channel impulse response is static. However, the usual case is that both the antennas and surrounding objects are moving, and therefore the impulse response is time-variant $(h(t, \tau))[56]$.

The channel impulse response groups the effect of the transmitting antenna, plus the propagation channel, plus the receiving antenna. A change in any of the antennas, thereby changes the impulse response. However, the physical propagation channel remains constant. This physical channel propagation, independent of the antenna (and thus the system) can be characterized by the so-called double-directional impulse response.

$$
h(t, \tau)=\int_{4 \pi} \int_{4 \pi} g_{T}\left(\varphi_{T}\right) p\left(t, \tau, \varphi_{R}, \varphi_{T}\right) g_{R}\left(\varphi_{R}\right) d \varphi_{T} d \varphi_{R}
$$

Where $\varphi_{T}$ represents the angle (and therefore the direction) where the transmitting antenna radiates, $\varphi_{R}$ represents the incoming angle of the wave at the receiving position, and $g_{R}\left(\varphi_{R}\right)$ and $g_{T}\left(\varphi_{T}\right)$ are the complex gains of the antennas. Then integral sums the radiation of the whole system over all the possible directions.

Assuming a finite $K$ number of paths, transforms the integral in a summation, with the consequent reduction in complexity.

$$
h(t, \tau)=\sum_{i=1}^{K} g_{T}\left(\varphi_{T}\right) p\left(t, \tau, \varphi_{R}, \varphi_{T}\right) g_{R}\left(\varphi_{R}\right)
$$


The impulse response described above is applicable to single polarized antennas. A more general case is when both transmitting and receiving antennas are dual polarized. Then we can write a matrix of impulse responses with all the possible combinations between polarisations.

$$
h(t, \tau)=\left[\begin{array}{ll}
h_{v v}(t, \tau) & h_{v h}(t, \tau) \\
h_{h v}(t, \tau) & h_{h h}(t, \tau)
\end{array}\right]
$$

Everything we have seen so far is valid for the case where there is only one antenna in the transmitting part, and another one in reception (SISO). However, to describe the full MIMO channel, we need to characterize the impulse response of all possible combinations of antennas. Thus, for the general case of $M$ transmitters and $N$ receiving antennas, the MIMO channel is characterized by an $M x N$ dimension matrix, which includes all the double-directional impulse responses for all the single antenna combinations.

$$
h(t, \tau)=\left[\begin{array}{ccc}
h_{11}(t, \tau) & \cdots & h_{1 M}(t, \tau) \\
\vdots & \ddots & \vdots \\
h_{N 1}(t, \tau) & \cdots & h_{N M}(t, \tau)
\end{array}\right]
$$

where $h_{n m}(t, \tau)$ represents the time-variant impulse response between the input of the $m^{\text {th }}$ transmit antenna and the output of the $n^{\text {th }}$ receive antenna. If polarization diversity is present on the antennas, then each impulse response $h_{n m}(t, \tau)$ must be substituted by the $2 \times 2$ matrix defined in eq. (31)

A definition of the appropriate MIMO channel matrix (including the propagation impulse response) to be used during wireless devices characterization has been a hot topic for research in the wireless industry over the last years, and nowadays it is still not clear what are the most significant propagation environments in which devices should be analysed. Moreover, the ability to perform such tests in a controlled lab environment rather than in drive tests in actual environments is attracting interest. Drive tests suffer from high costs and poor repeatability, drawbacks which can be eliminated if the same tests can be performed in a lab. For that reason, there exist a number of channel models that have been proposed. In the next section it is shown a short classification of them.

\subsubsection{Channel Model Classification}

A remarkable study on the different cannel models is presented in [57]. According to this study, channel models can be separated in two main categories (shown in Figure 16), depending on whether they include or not the antenna properties of the system. Below it is shortly described each of these two categories: 
- $\quad$ Physical channel models characterize the propagation environment from an electromagnetic point of view. To this end, by propagation theory of electromagnetic waves, the bidirectional propagation channel that connects the positions of the transmitter and receiver is described. Thus, they are evaluated parameters inherent to the physical channel (regardless of transmitter or receiver systems) as the complex amplitude, DS, the AoA or AoD. This type of channel models is independent of parameters such as antenna radiation pattern, number of antennas, polarization... Channel models based on ray tracing, or the extension of Saleh-Valenzuela, are examples of this type of models.

- $\quad$ Analytical channel models, approach the problem from another perspective. What they do is to characterize the transfer function between the transmitters and receivers antennas, individually. Thus, the analysis is not in terms of electromagnetic wave propagation. On the contrary it produces a matrix that groups MIMO impulse responses of all possible combinations of antennas. Other authors propose to perform this characterization of the channel matrix using the correlation between the different antennas. [58] [59]

Apart from the models listed in the above categories, some international organizations have proposed a number of channel models for the purpose of comparing different wireless systems in order to define some reproducible conditions. These models are based on field measurements made in real environments such as streets, indoors and rural environments [10] [11] [12] [13] [60]. The objective of this type of models is to clearly define a reference environment where wireless devices can be tested the same way in different laboratories and with different methodologies. 


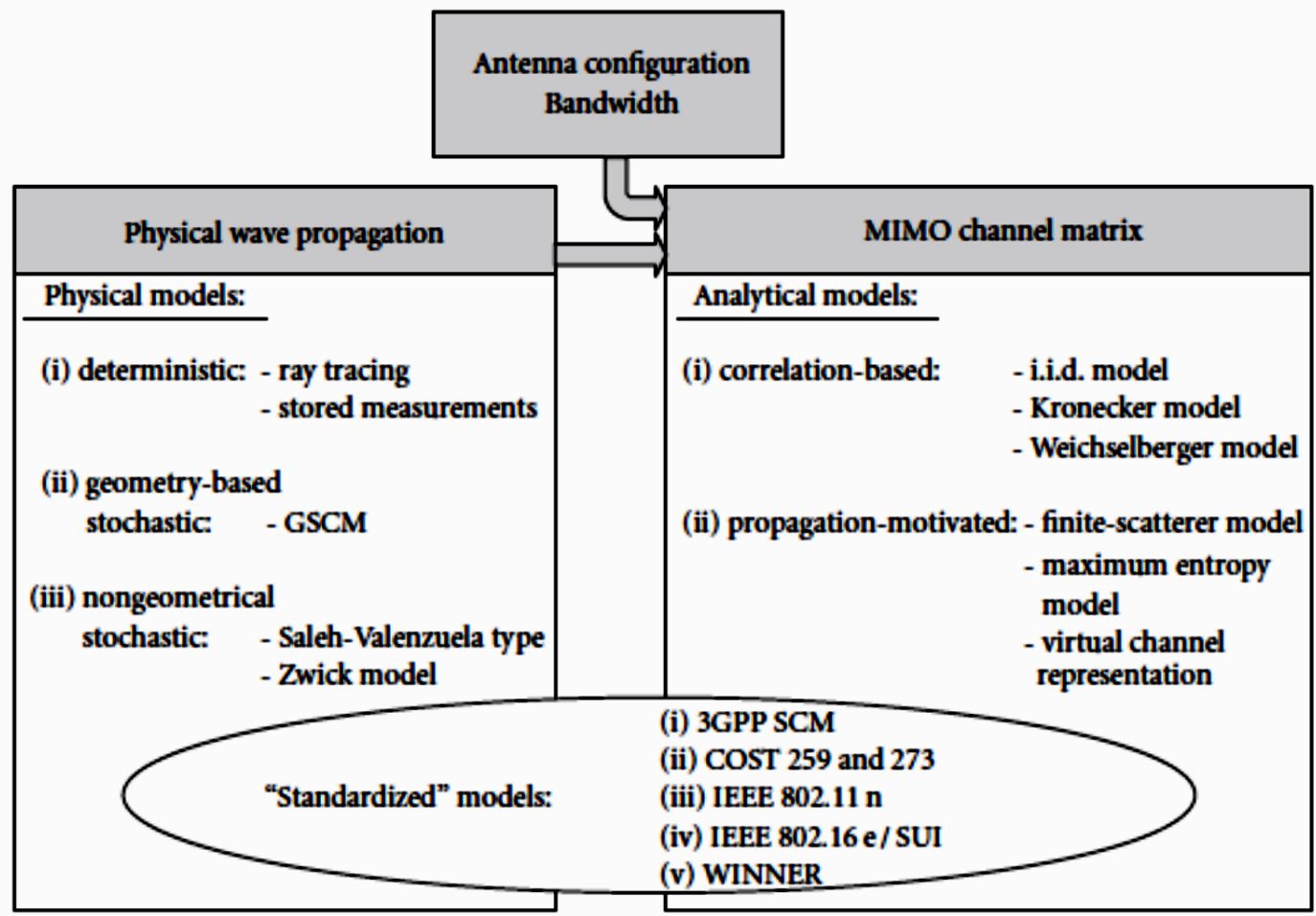

Figure 16. Overview of the different types of cannel models currently used. SOURCE: [57]

\subsection{Standardized Channel Models Commonly Used in Industry}

\subsubsection{GPP Spatial Channel Model (SCM)}

The SCM family of channel models was developed by 3GPP in order to satisfy the need of channel models serve as reference for measurements in outdoors. Three scenarios are defined, which aim to simulate the most typical use conditions of use that are experienced under real situations.

-"Urban macrocell" simulates the scenario where a user is in a very reflective

(urban) environment, but where there is a certain distance to the BS.

-"Suburban macrocell" simulates the case where the user is in a non-reflective (suburban or rural) scenario, and therefore the direct component prevails over the reflected components.

-"Urban microcell" simulates the case where a user in a reflective scenario and is being serviced by a BS located within walking distance of it (typical of a microcell).

SCM models are used so that the system simulation is performed as a sequence of drops, where a drop is a run of the channel model during a very short time. The duration is short enough, so that it can be assumed that the AS, mean AoA, DS and shadowing are constant throughout the drop. These models have a geometric and a stochastic component. The geometric component is that the position of the DUT with 
respect to the $\mathrm{BS}$, the orientation of the antennas and the direction of motion, are calculated randomly at the beginning of each drop.

The three models consist of 6 main multipath components. However, both the delay and the power of each component are calculated stochastically according to the probability density function associated with each variable. The angular dispersion implementation is performed by introducing 20 subpaths for each of the 6 main components, with the same delay than those but different DoA.

The SCM model also incorporates additional features such as the incorporation of the polarization of the antennas, a far scatterer clusters effect (which represents the bad-urban case where additional clusters are seen in the environment), a LoS case for the urban microcell scenario, and an additional angular distribution at the DUT position emulating a urban street canyon scenario.

The shortcomings of this model are mainly three. Firstly, it is not supposed to work for higher bandwidths than $5 \mathrm{MHz}$, so it is not suitable for the new coming $4 \mathrm{G}$ standards, which use up to $20 \mathrm{MHz}$. Secondly, the SCM model was designed for a CDMA systems working at $2 \mathrm{GHz}$, so accuracy is not guaranteed for other frequency bands. Finally, the Rician $k$-factor emulating the LoS effect at the receiver position can only be introduced for the microcell case. In order to overcome those limitations the extended SCM (SCME [11]) models were proposed.

\subsubsection{Extended 3GPP Spatial Channel Model (SCME).}

The SCME model proposed the extension of some of the parameters proposed by the SCM model, maintaining the basic idea of the original models. With the arrival of the $4 \mathrm{G}$ standards, an extension of the channel bandwidth applicability is required to the channel model. The SCME model proposes to add intra-path DS following a onesided exponential function. This approach is based in the methodology proposed by Saleh and Valenzuela [61] for the modeling of indoor environments.

Another important extension included in the SCME model is the calculation of the pathloss, in order to extend the frequency applicability to the $5 \mathrm{GHz}$ band. The SCM path-loss model is based on the COST-Hata- Model [62] for Suburban and Urban Macro and the COST-Walfish-Ikegami-Model (COST-WI) [62]. Instead, the SCME model proposes the use of the COST-WI model approximation for all the scenarios, due to its distance range $(0.02-5 \mathrm{~km})$ is more appropriate to the current standards use. (The Hata Model was calculated for GSM with a distance range of up to $20 \mathrm{Kms}$ ) The proposed parameters for the different scenarios are:

- $\quad$ BS antenna height: Macro $32 \mathrm{~m}$, Micro $10 \mathrm{~m}$; 
- $\quad$ Building height: Urban 12 m, Suburban 9 m;

- $\quad$ Building to building distance: $50 \mathrm{~m}$, street width: $25 \mathrm{~m}$,

- DUT antenna height: $1.5 \mathrm{~m}$,

- Orientation: $30^{\circ}$ for all paths,

- "Macro" scenarios: medium sized city / suburban centers,

- $\quad$ "Micro" scenarios: metropolitan.

The applicability of the LoS (or Rician $k$-factor) to all the environments is crucial in order to properly emulate some environments where the direct component is prevailing over the reflected part of the signal. The calculation of the $k$-factor is done according to the models described in [63]. Used parameters in the SCME model are; BS heights of approximately $20 \mathrm{~m}$, DUT antenna height $1.5 \mathrm{~m}$, DUT beam-width: $360^{\circ}$, and selection of season: summer, which results in the following equation:

$$
k=15.4-5.0 \log _{10}(d)
$$

where $d$ is the BS-DUT distance in $\mathrm{m}$, and $k$ is in $\mathrm{dB}$.

Finally, an important characteristic proposed by the SCME model is the incorporation of a fixed set of delay taps and angular parameters, instead of the stochastic FDP estimation proposed in the original SCM. This extension is especially important for some methodologies, since it allows an important reduction in the complexity of the equipment. Proposed values are shown in Table 6.

Table 6. Most Relevant Parameters in the SCME CanNel Models. Source: [11]

\begin{tabular}{|c|c|c|c|c|c|c|c|c|c|}
\hline \multicolumn{2}{|l|}{ Scenario } & \multicolumn{2}{|c|}{ Suburban Macro } & \multicolumn{4}{|c|}{ Urban Macro } & \multicolumn{2}{|c|}{ Urban Micro } \\
\hline \multirow{6}{*}{$\begin{array}{l}\text { Power-delay parameters: } \\
\text { relative path power }(\mathrm{dB}) / \\
\text { delay }(\mu 5)\end{array}$} & 1 & 0 & 0 & \multicolumn{2}{|c|}{0} & \multicolumn{2}{|c|}{0} & 0 & 0 \\
\hline & 2 & -2.6682 & 0.1408 & \multicolumn{2}{|c|}{-2.2204} & \multicolumn{2}{|c|}{0.3600} & -1.2661 & 0.2840 \\
\hline & 3 & -6.2147 & 0.0626 & \multicolumn{2}{|c|}{-1.7184} & \multicolumn{2}{|c|}{0.2527} & -2.7201 & 0.2047 \\
\hline & 4 & -10.4132 & 0.4015 & \multicolumn{2}{|c|}{-5.1896} & \multicolumn{2}{|c|}{1.0387} & -4.2973 & 0.6623 \\
\hline & 5 & -16.4735 & 1.3820 & \multicolumn{2}{|c|}{-9.0516} & \multicolumn{2}{|c|}{2.7300} & -6.0140 & 0.8066 \\
\hline & 6 & -22.1898 & 2.8280 & \multicolumn{2}{|c|}{-12.5013} & \multicolumn{2}{|c|}{4.5977} & -8.4306 & 0.9227 \\
\hline \multicolumn{2}{|l|}{ Resulting total DS ( } & \multicolumn{2}{|c|}{0.231} & \multicolumn{4}{|c|}{0.841} & \multicolumn{2}{|c|}{0.294} \\
\hline \multicolumn{2}{|l|}{ Path AS at BS, MS (deg) } & \multicolumn{2}{|c|}{2,35} & \multicolumn{4}{|c|}{2,35} & \multicolumn{2}{|c|}{5,35} \\
\hline \multirow{6}{*}{$\begin{array}{l}\text { Angular parameters: } \\
\text { AoA (deg)/ } \\
\text { AoD (deg) }\end{array}$} & 1 & 156.1507 & -101.3376 & 65.7489 & 81.9720 & 76.4750 & -127.2788 & 0.6966 & 6.6100 \\
\hline & 2 & -137.2020 & -100.8629 & 45.6454 & 80.5354 & -11.8704 & -129.9678 & -13.2268 & 14.1360 \\
\hline & 3 & 39.3383 & -110.9587 & 143.1863 & 79.6210 & -14.5707 & -136.8071 & 146.0669 & 50.8297 \\
\hline & 4 & 115.1626 & -112.9888 & 32.5131 & 98.6319 & 17.7089 & -96.2155 & -30.5485 & 38.3972 \\
\hline & 5 & 91.1897 & -115.5088 & -91.0551 & 102.1308 & 167.6567 & -159.5999 & -11.4412 & 6.6690 \\
\hline & 6 & 4.6769 & -118.0681 & -19.1657 & 107.0643 & 139.0774 & 173.1860 & -1.0587 & 40.2849 \\
\hline
\end{tabular}

\subsubsection{WINNER channel models}

The Winner Channel models [12] [13] emerged with the aim of solving the problems presented by the SCM models, as well as to add more usage scenarios, to 
overcome the difficulties to evaluate all possible usage scenarios with only the three scenarios proposed by SCM. These models are designed using a geometry-based stochastic channel modeling approach, which allows creating of an arbitrary double directional radio channel model. The channel models are antenna independent (since it is a physical model). As well as in the SCM models, the channel parameters are determined stochastically, based on statistical distributions extracted from channel measurement.

In the phase I of the WINNER project, they proposed a generic model based on the same principles than SCM models, but extending the bandwidth and the number of scenarios (six in total). The model implements a ray-based double-directional model multi-link independent of the antenna, which makes the model scalable and capable of modeling MIMO channels. The model was taken from a measurement campaign at 2 and $5 \mathrm{GHz}$ The following propagation models were defined:

- Indoor.

- Typical urban micro-cell.

- Typical urban macro-cell.

- Sub-urban macro-cell.

- $\quad$ Rural macro -cell.

- $\quad$ Stationary feeder link.

In phase II of the WINNER project, they made some extensions to the original WINNER models. One of the most important is the extension of the frequency range used so that channel models are applicable in the range 2-6 GHz. There were also added more scenarios, based on measurement campaigns, for a total of 13 . The covered propagation scenarios are:

- $\quad$ A1 - Indoor office

- $\quad$ A2 - Indoor to outdoor

- B1 - Urban micro-cell

- $\quad$ B2 - Bad Urban micro-cell

- B3 - Indoor hotspot

- B4-Outdoor to indoor

- $\quad$ B5 - Stationary Feeder

- C1 - Suburban macro-cell 
- $\quad$ C2 - Urban macro-cell

- $\quad$ C3 - Bad urban macro-cell

- C4 - Urban macro outdoor to indoor

- $\quad$ D1 - Rural macro-cell

- $\quad$ D2 - Moving networks

WINNER II models also add features such as the extension of the AoA in elevation plane, the possibility of incorporating a LoS component to each of the 13 scenarios and the inclusion of polarization in the channel model. The models are scalable from a SISO or MIMO link to a multi-link MIMO scenario. This family of models can be applied to any wireless system operating in the frequency bands covered by the 2-6 GHz range, with up to $100 \mathrm{MHz}$ bandwidth.

There is a dependency of the models with frequency, due to the pathlosses. For WINNER models, the pathlosses model was obtained from a measurement campaign at 2 and $5 \mathrm{GHz}$, as well as using some results obtained in the literature (like OkumuraHata model), and therefore it is necessary to perform a frequency scale of the losses for the remaining frequencies in the range $2-6 \mathrm{GHz}$. The general formula for the pathloss calculation is:

$$
P L=A \cdot \log _{10}(d[m])+B \cdot C \cdot \log _{10}\left(\frac{f_{c}[\mathrm{GHz}]}{5.0}\right)+X
$$

where $d$ is the distance between the transmitter and the receiver in $[\mathrm{m}], f_{c}$ is the system frequency in $[\mathrm{GHz}]$, the fitting parameter $A$ includes the path-loss exponent, parameter $B$ is the intercept, parameter $C$ describes the path loss frequency dependence, and $X$ is an optional environment-specific term.

Another interesting feature of WINNER channel models is the ability to extend the 13 models proposed between LOS and NLOS conditions. This option allows introducing a Rician $k$-factor to any of these scenarios, modeling situations in which the rest of the channel settings remain constant, but the condition of visibility does not. For example, in the A1(indoor) and B1 (urban microcell) scenarios, transitions from LoS to NLoS can occur as a result of the MS turning from the corridor or street in which the BS is located into a perpendicular corridor or street.

As it happened with the SCM channel, it was soon identified the need for simplified versions, to facilitate the application of models to specific measurement methodologies with a reasonable level of complexity. In the simplified model each cluster is composed of 20 rays with fixed offset angles and identical power, and the same or close delay. In the case of cluster where a ray of dominant power exists (LoS 
Where $\tau$ is the PDP mean value,

$$
\bar{\tau}=\frac{\int_{-\infty}^{\infty} P D P(\tau) \tau d \tau}{\int_{-\infty}^{\infty} P D P(\tau) d \tau}
$$

The rms DS has attracted much interest in the channel model characterization, since some studies has demonstrated that under some circumstances there is a strong relation between error probability and the delay dispersion. That makes this parameter very important for the channel model characterization, when those channels are intended to be used with active wireless devices, since delay dispersion has a strong impact on the ability of the system to properly demodulate a signal.

Many channel models are defined by the summation of several delay taps (Dirac deltas) for each of the multipath components reaching the receiver. However, many studies confirm that the shape of these taps is not a Dirac delta but follows an exponential model. Thus a delay component centered in the time $\tau_{0}$, can be modeled as:

$$
\operatorname{PDP}(\tau)=\left\{\begin{array}{cc}
\exp \left(-\frac{\left(\tau-\tau_{0}\right)}{D S_{r m s}}\right) & \tau \geq \tau_{0} \\
0 & \text { otherwise }
\end{array}\right.
$$

Where $D S_{r m s}$ is referred to the rms DS created by the multipath cluster situated in $\tau_{0}$

Some rms DS values found in the literature are [64]

- $\quad$ Indoor residential buildings: 5- 30 ns.

- Indoor office environments: 10-300 ns.

- $\quad$ Factories and airport halls: 50 to 200 ns.

- Microcells: 5-100 ns (for LoS situations) to 100-500 ns (for NLoS).

- Tunnels and mines: 20-100 ns.

- Typical urban and suburban environments: these show delay spreads between 100 ns $3 \mu \mathrm{s}$.

- $\quad$ Bad urban and hilly terrain environments: up to $50 \mu \mathrm{s}$.

These values show the big differences that can be found in real environments, and justifies the importance of a proper definition of channel models, in order to prevent bad behaviors of wireless systems in real environments. 


\subsubsection{Fading Signal Strength Distribution}

The statistical behaviour of the fading experienced by an electromagnetic signal in real environments represents an important tool for analysing and designing wireless systems. As shown in [64], in an electromagnetic environment with limited noise, the behaviour of the system is determined by the received field strength. The field strength has a stochastic behaviour, and therefore must be analysed in statistical terms. Thus the PDF, both in the field amplitude, and phase, is one of the most important parameters when characterizing the fading of an environment.

However, an environment that has good statistically speaking conditions (large mean field strength), does not mean that it will be possible to maintain the communication established at every moment. To quantify this, it is also used the CDF, which represents the probability that the signal level does not drop below a certain value. The CDF can be easily obtained by the undefined integral of the PDF, for a given fading function.

\subsubsection{Rayleigh}

The most typical statistical behaviour that we find in real multipath environments (especially in urban and indoor cases), is that one where both amplitude and phase follows a Gaussian distribution. Therefore, it is possible to mathematically demonstrate that a variable with amplitude $r$ and phase $\gamma$ follows a distribution [64]:

$$
p d f_{r, \gamma}(r, \gamma)=\left\{\begin{array}{cc}
\frac{r}{2 \pi \sigma^{2}} \exp \left(-\frac{r^{2}}{2 \sigma^{2}}\right) & 0 \leq r \leq \infty, 0 \leq \gamma \leq 2 \pi \\
0 & \text { otherwise }
\end{array}\right.
$$

As it can be appreciated, there is no dependency with the phase, so the phase is uniform distributed in the range $0-2 \pi$.

Some of the advantages of this distribution model for wireless systems are, for example, that depends only on one parameter $r$ (directly related to the received mean power), which greatly facilitates the modelling of real channels with this distribution.

This type of field distribution is also the fading distribution naturally generated by a conventional MSRC, which has also greatly extended the use of this model.

\subsubsection{Rician}

If we introduce a LoS component a Rayleigh distributed environment, it is obtained the so-called Rician distribution [40]. In that case, the real part of signal follows a nonzero-mean Gaussian distribution, while the phase follows a zero-mean Gaussian distribution. That can be written as: 


$$
p d f_{r, \gamma}(r, \gamma)=\left\{\begin{array}{cc}
\frac{r}{2 \pi \sigma^{2}} \exp \left(-\frac{r^{2}+A^{2}-2 r A \cos (\gamma)}{2 \sigma^{2}}\right) & 0 \leq r \leq \infty, 0 \leq \gamma \leq 2 \pi \\
0 & \text { otherwise }
\end{array}\right.
$$

where $A$ is the amplitude of the LoS component.

It is possible to isolate amplitude and phase from each other, as it is showed in [64].

- $\quad$ Amplitude PDF:

$$
p d f_{r}(r)=\frac{r}{\sigma^{2}} \exp \left(-\frac{r^{2}+A^{2}}{2 \sigma^{2}}\right) I_{0}\left(\frac{r A}{\sigma^{2}}\right) \quad 0 \leq r \leq \infty
$$

Where $I_{0}(x)$ is the modified Bessel function of the first kind, zero order.

- $\quad$ Phase PDF:

$$
p d f_{\gamma}(\gamma)=\frac{1+\sqrt{\pi K} e^{K \cos ^{2}(\gamma)} \cos (\gamma)(1+\operatorname{erf}[\sqrt{K} \cos (\gamma)]}{2 \pi e^{K}} \quad 0 \leq \gamma \leq 2 \pi
$$

where $k$ is the ratio of power between the LoS component and the scattered component, commonly known as Rician $k$-factor,

$$
K=\frac{A^{2}}{2 \sigma^{2}}
$$

and $\operatorname{erf}(x)$ is the error function

$$
\operatorname{erf}(x)=\left(\frac{2}{\sqrt{\pi}}\right) \int_{0}^{x} \exp \left(-t^{2} d t\right)
$$

\subsubsection{Nakagami}

Another function that is commonly used in the emulation of wide band channel models is the Nakagami distribution [65].

$$
p d f_{r}(r)=\frac{2}{\Gamma(m)}\left(\frac{m}{\Omega}\right)^{m} r^{2 m-1} \exp \left(-\frac{m}{\Omega} r^{2}\right) \quad r \geq 0, m \geq 0.5
$$

Where $\Gamma(m)$ is the Euler's Gamma function, and the parameter $m$ is,

$$
m=\frac{\overline{\Omega^{2}}}{\overline{\left(r^{2}-\bar{\Omega}\right)^{2}}}
$$

Being $\Omega$ the mean square value of $r$.

Nakagami and Rician distributions are quite similar in shape, but the main difference they present is that Nakagami model fits better some Ultra Wide Band (UWB) channels [66], while Rician model is more appropriate for environments with LoS. 
However it is shown in [64] that there exists a direct relation to approximate a Rician model from a Nakagami or vice-versa:

$$
\begin{aligned}
& m=\frac{(k+1)^{2}}{(2 k+1)} \\
& k=\frac{\sqrt{m^{2}-m}}{m-\sqrt{m^{2}-m}}
\end{aligned}
$$

\subsubsection{Lognormal}

Another signal distribution that has been observed in some real environments, such as UWB channels [66] and "On-Body" channels for personal area networks (PAN) [67] is the log-normal distribution, which is a density function which presents a Gaussian shape when plotted in a logarithmic scale. The PDF is given by [64],

$$
p d f_{r}(r)=\frac{20 / \ln (10)}{r \sigma \sqrt{2 \pi}} \exp \left[-\frac{\left(20 \log _{10}(r)-\mu_{d B}\right)^{2}}{2 \sigma^{2}}\right] \quad 0 \leq r \leq \infty
$$

Where $\sigma$ is the standard deviation and $\mu_{d B}$ is the mean of the values of $r$ expressed in $\mathrm{dB}$. Typical measured values of $\sigma$ go from 4 to $10 \mathrm{~dB}$. 


\section{Study on the Effect of User Presence over Wireless Devices Performance.}

\subsection{Influence Analysis over Passive Antenna Parameters}

User influence over multiple antenna device performance has been a topic for research in the last few years, since it is well known that a user in the vicinity of a wireless device affects the propagation conditions that the device is experiencing. This effect is well known for SISO communications, consisting of a degradation of radiation performance due to the losses introduced by the user. This effect is usually quantified by the changes in radiation efficiency and absorbed power [68].

In spite of this knowledge, it is still not clear what the consequences are when it comes to multiple antenna devices, since those base their enhanced capabilities on a rich field distribution in terms of signal paths. Numerous studies have been performed over the last years, agreeing to the fact that the effect of the human body is more complex in MIMO terminals than in traditional ones [21] [69] [70] [71]. Depending on the antenna design and user influence, both increase and decrease of correlation and DG can be found in the literature [72].

In this chapter the performance of different handsets is analysed. All the handsets used within this study consist of two antennas, in order to implement diversity at the receiving terminal end of the link (SIMO - Single Input Multiple Output). This SIMO configuration is relevant for this study since it is one of the normal OTA test cases for the new communication standards (HSDPA and LTE).

Four different handsets are used for this study. Two of them work at low frequency (700-780 MHz) and the other two work at a higher frequency (2620-2690 MHz). For each band, there is one handset with a good antenna solution and the other one has been designed on purpose to have a bad MIMO behaviour. 
Two samples of the same handset model are used with dimensions $115 \mathrm{~mm} \times 65$ $\mathrm{mm}$, working at $700 \mathrm{MHz}$. The two test objects are referred to as Prototype $A$ and Prototype B. Prototype A has 2 monopole antennas located at each short side of the handset. The two monopoles excite the chassis in the same way, so high correlation is expected. Prototype $B$ includes one monopole located at one of the short sides of the handset, and a notch antenna located along the long side of it. These two antennas excite the chassis in a different way, and consequently, correlation is lower.

Two terminal antenna models are used with dimensions $100 \mathrm{~mm} \times 40 \mathrm{~mm}$, working at $2600 \mathrm{MHz}$. The two test objects are referred to as Prototype $\mathrm{C}$ and Prototype $\mathrm{D}$. Both Prototype $\mathrm{C}$ and Prototype $\mathrm{D}$, consist of a ground plane and two Planar Inverted $\mathrm{F}$ Antennas (PIFA) fed by coaxial cables. Prototype $\mathrm{C}$ is designed to have high correlation on purpose, by slightly connecting the patches of the two single PIFA antennas.

Measurements were carried out using a MSRC (length $3 \mathrm{~m}$, width $2.45 \mathrm{~m}$, and height $2.45 \mathrm{~m}$ ) located at SP Technical Research Institute of Sweden, Borås, Sweden. The MSRC comprises an electronically controlled turn table, as well as a rotational zigzag stirrer placed in a corner of the metallic cavity. The MSRC shielding effectiveness of this MSRC is $100 \mathrm{~dB}$. The proposed setup is shown in Figure 17.

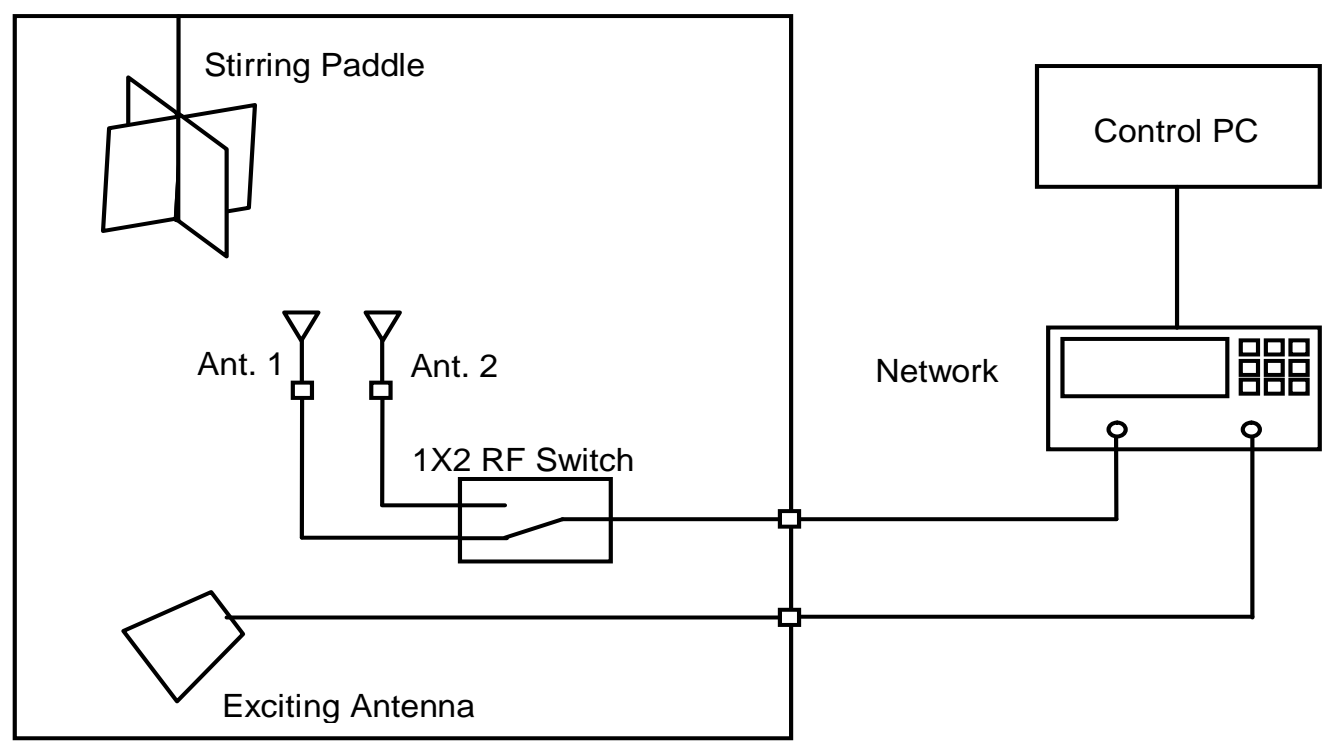

Figure 17. Scheme of the MSRC setup.

Three different scenarios (no user, head phantom and real person) are used to simulate different effects of the user on the radiation performance of the antennas.

No user scenario is the classical scenario generated in a MSRC, with an isotropic and Rayleigh distributed field strength at the device. In this scenario the antennas under test were placed over a low loss dielectric foam piece, in order to avoid as much as possible the effect of the holder. 
Head phantom is the commonly used scenario to estimate the behaviour of a device including user influence, that is, device attached to the cheek of the phantom and aligned between the ear point and the mouth point (Fig. 18).

Figure 18. Measurement setup with the presence of the head phantom and the device placed in talk position.

Real Person scenario is performed with the introduction of a real person inside the chamber, holding the device with the hand simulating talk position, in the same way as in the Head Phantom scenario. The person is sitting on a chair placed in the centre of the chamber, in order to have the antenna in a similar place than in the other scenarios.

No User is used as a reference case. This scenario, although useful, is not intended to be realistic. With the introduction of a head phantom, the effect of the user head on the antenna is included. The head phantom affects the close environment of the antenna, but it is still an intermediate approach to a real user influence simulation, since a head phantom does not block all the incident waves that a real person would. This is the motivation of the Real Person scenario.

\subsubsection{Correlation}

For the purpose of these measurements, a stirring sequence of 400 positions is selected (100 stirrer position $\times 4$ turn table positions), and the number of points of the VNA used to perform the $S_{21}$ measurements is set to 401 . Equation seen in Chapter 3 for antenna correlation is applicable. 
In total, the number of measurements is: [3 scenarios $\times 100$ stirrer positions $\times 4$ turn table positions $\times 401$ frequency points $=481200$ samples]. The measurement of each of the scenarios took about 40 minutes, for each dual antenna. Some problems were detected due to the decorrelation introduced by the cables connected to the antennas. In order to avoid those problems, a RF switch is introduced between one of the connectors of the vector network analyzer and the antennas under test, so the $S_{21}$ can be measured for the two receiving antennas without changing the position of the cables.

Figs. 19 and 20 show the different correlation values obtained under the three different user influence scenarios, for antennas at both bands.
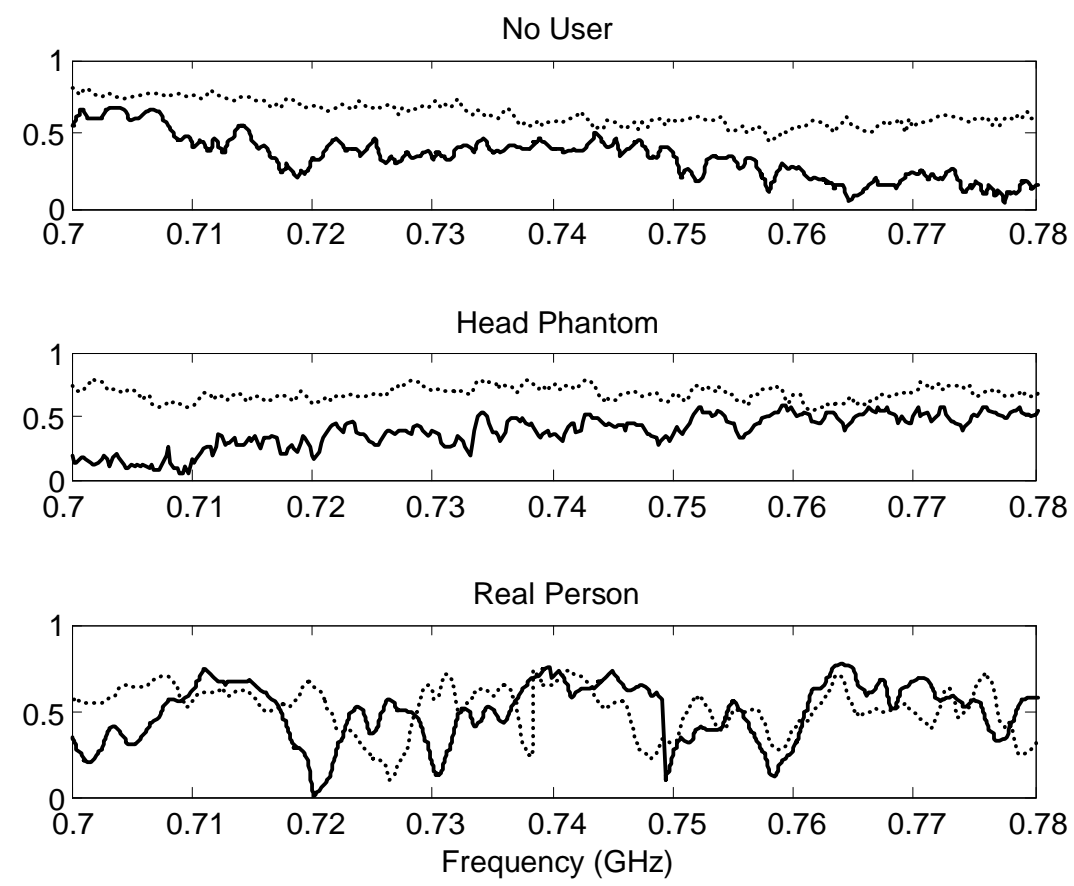

Figure 19. Correlation of the $700 \mathrm{MHz}$ band devices for the three different scenarios. The dotted line corresponds to Prototype A; solid line represents Prototype B. 

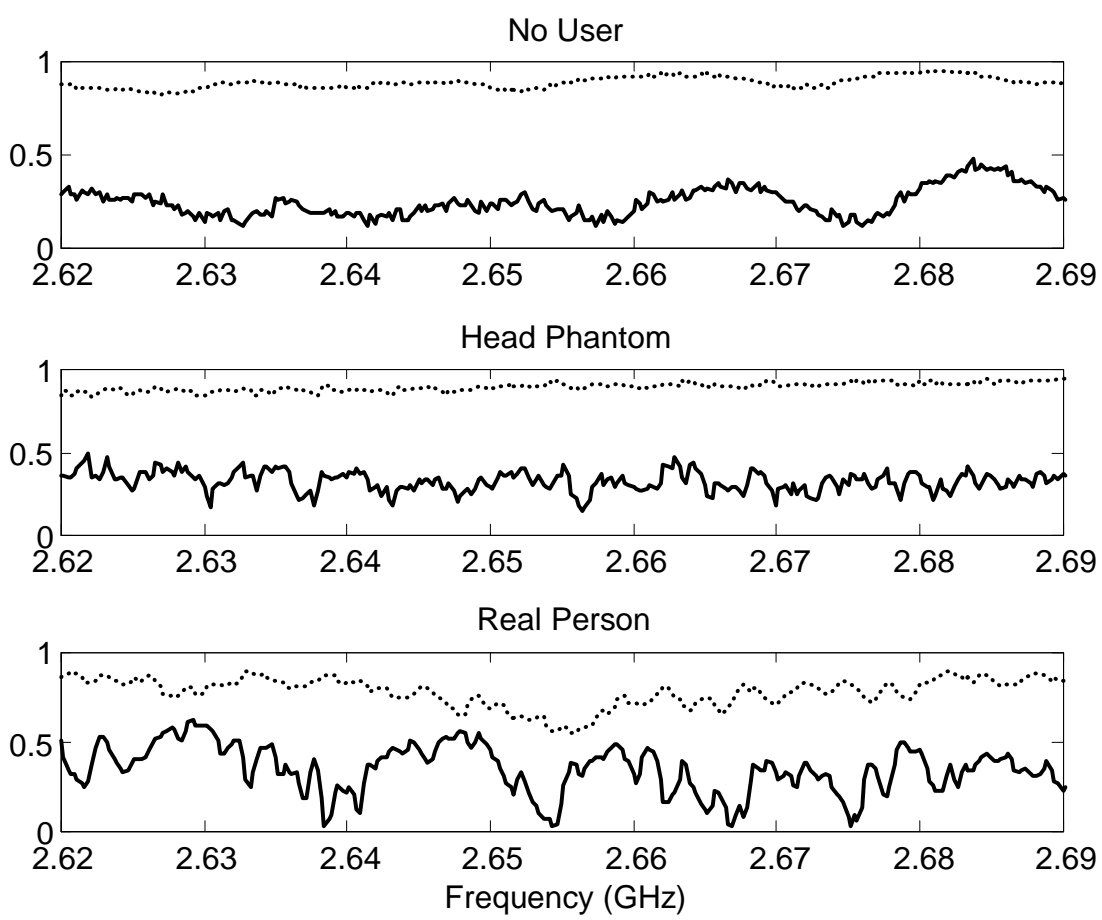

Figure 20. Correlation of the $2600 \mathrm{MHz}$ band devices for the three different scenarios. The dotted line corresponds to Prototype C; solid line represents Prototype D.

TABle 8. Correlation Results (MeAn)

\begin{tabular}{c|c|c|c|c}
\hline \hline Band & Prototype & No User & Head Phantom & Real Person \\
\hline \multirow{2}{*}{$700 \mathrm{MHz}$} & A & 0.68 & 0.67 & 0.52 \\
& B & 0.30 & 0.38 & 0.50 \\
\hline \multirow{2}{*}{$2600 \mathrm{MHz}$} & C & 0.88 & 0.88 & 0.76 \\
& D & 0.25 & 0.34 & 0.33 \\
\hline \hline
\end{tabular}

Table 9. Correlation Results (Standard Deviation)

\begin{tabular}{c|c|l|c|c}
\hline \hline Band & Prototype & No User & Head Phantom & Real Person \\
\hline \multirow{2}{*}{$700 \mathrm{MHz}$} & A & 0.100 & 0.051 & 0.133 \\
& B & 0.155 & 0.127 & 0.164 \\
\hline \multirow{2}{*}{$2600 \mathrm{MHz}$} & C & 0.026 & 0.031 & 0.058 \\
& D & 0.068 & 0.065 & 0.126 \\
\hline \hline
\end{tabular}

Correlation is clearly affected by the presence of the user. While the handsets present a well-recognized low and high correlation behaviour in the No User scenario, this difference becomes smaller as the presence of the user is more significant. 
It is also worth noticing the different impact depending on the frequency. Even though the effect is important at the higher frequency, it has a dramatic impact at the $700 \mathrm{MHz}$ band, where correlation becomes very similar in both test objects, and with large variations over the band.

\subsubsection{DG}

DG is one of the most recognized figures-of-merit when evaluating multiple antenna terminals. DG quantifies the improvement created by the existence of more than one antenna over a reference case. In this study, there is not used a reference antenna, instead each of the two receiving antennas are used as a reference (so there are two DG values for each prototype and scenario).

Several schemes can be used to combine the signals coming from the two antennas. Since the prototypes and antennas used in this study are intended to be part of complex wireless devices, the MRC [37] [73] scheme is used, which is common for these kind of devices. Thus, the compound signal can be expressed as,

$$
S_{21, c}=\sqrt{\left|S_{21,1}\right|^{2}+\left|S_{21,2}\right|^{2}}
$$

After this, DG can be calculated at the $99 \%$ signal reliability levels, according to,

$$
D G_{k}=10 * \log _{10}\left(\frac{\left|S_{21, c, 99 \%}\right|^{2}}{\left|S_{21, k, 99 \%}\right|^{2}}\right)
$$

where two values are calculated $(k=1$ and $k=2)$ with each of the two antennas serving as the non-diversity reference case. The reason for doing this, rather than using one specific antenna as the reference, is that we hereby avoid assuming which antenna would be used in a non-diversity case.

Table 10 shows the performance of the devices for a signal reliability of $1 \%$. Efficiency of antennas is not taken in account for the DG calculations, which means that ADG is calculated [74] [75].

Table 10. Apparent Diversity Gain Results (Decibels).

\begin{tabular}{l|c|c|c|c|c}
\hline \hline Band & Prototype & $\mathbf{k}$ & No User & Head Phantom & Real Person \\
\hline $\mathbf{7 0 0} \mathrm{MHz}$ & A & 1 & 6.38 & 6.98 & 6.71 \\
& & 2 & 6.68 & 7.01 & 6.89
\end{tabular}




\begin{tabular}{l|l|l|l|l|l} 
& B & 1 & 8.21 & 8.40 & 7.35 \\
& & 2 & 8.37 & 8.51 & 7.44 \\
\hline $\mathbf{2 6 0 0} \mathrm{MHz}$ & $\mathbf{C}$ & 1 & 5.52 & 5.75 & 7.28 \\
& $\mathbf{D}$ & 1 & 8.63 & 5.77 & 7.40 \\
& & 2 & 8.88 & 8.10 & 8.38 \\
\hline \hline
\end{tabular}

In Table 10, $A D G$ results in $d B$ are presented for the different scenarios. Low correlation handsets present a reduction in diversity gain when the user influence becomes more important. The effect is the opposite for the high correlated handsets.

The changes in the real user scenario are more pronounced than those produced on stage with the head phantom. This is because the head phantom represents an average user and the actual users have their own characteristics, given the results we can guess that each terminal will behave differently depending on the end user. It can therefore be interesting to model different real users.

This effect is in line with the correlation that both devices present under the three different scenarios. As we have seen before, the correlation between antennas is decreased in high correlated devices when they are under user influence. This effect can actually lead to a better MIMO performance of the device under user influence, compared to the performance the device has under No User scenario. Even more so, we can see how the DG results become very similar for both bad and good devices, in the case of a real person influence. It then seems that the real effect of a user on DG is the equalization of device performance, and not necessarily deterioration.

\subsection{Influence Analysis over Active Wireless Devices}

\subsubsection{HSDPA Data Throughput}

\subsubsection{OTA Measurements}

Some studies have already been published analyzing HSDPA devices in real conditions, but they focus their attention on evaluating the communication standard more than evaluating the device behaviour [76] [77] [78] [79]. In [80], HSDPA performance is evaluated for some different antenna schemes using two dipoles. 
However, there is a lack of studies evaluating commercial devices under real channel conditions.

In this section it is performed a study to characterize how the user influence affects the final performance of a real HSDPA device in terms of data throughput. As in active devices the channel model has very important impact on the device performance, measurements are repeated in two different channel models based on the WINNER II models defined in Chapter 4.

User influence on antenna properties is one of the most important causes degrading the throughput performance of a given DUT [72] [81] [82] [71]. The first part of the study focuses on the models without any influence of the user. In the second part, the study incorporates the effect that the user has on the performance of the device, and considers whether or not this effect is similar for different channel models.

In this study we demonstrate how some of these realistic channel models can be implemented in measurements using a MPS, which is an anechoic chamber based technique (as seen in chapter 2). This technique is used, because it is one of the most accepted technologies to implement spatial channel models, since they allow to carefully controlling the angles of the incoming paths. Two different channel models are studied based on the Winner II models, as defined in [13].

The novelty in the presented work compared to previous work is both the implementation of channel models in the MPS, rather than using a uniform signal distribution, and the use of a real person in an MPS for representation of user influence.

The MPS used during this study [83] [84] [85] [86] comprises 16 antennas (8 vertically and 8 horizontally polarized) surrounding DUT. Each signal path has different delay and power level as well as a phase shifter that allows emulation of Doppler shift and thereby also fast fading. The whole array is placed in an anechoic chamber. As the MPS is not bi-directional, another antenna is placed inside the anechoic chamber to maintain the uplink part of the communication.

A commercial mobile phone is used for this study. The device has HSDPA category 8 capability, and $\mathrm{H}$-Set 3 reference channel and Level Set 3 should be used to perform this study, according to the definition in [6]. 16-QAM modulation is used due to its higher sensitivity to fading conditions, compared with QPSK. Other testing conditions are extracted from [8].

The FoM for current and future cellular standards (HSDPA, LTE...) is commonly accepted to be the throughput, which stands for the total data flow over a given time, and which is usually measured in bits per second (bps). Measurements are carried out 
using a Rohde\&Schwarz CMU-200 BSE. Channel 10562 of HSDPA Band I is used (2110 $\mathrm{MHz}$.

Downlink performance degradation under fading conditions is measured by the BLER. BLER is estimated according to the definition in [6], and gives, together with the maximum throughput, the actual throughput.

\subsubsection{Winner II Channel Models Implementation}

This work includes implementing realistic channel models to the MPS. In this work we have used two of the Winner II models seen in Chapter 4, the B1LoS Urban MicroCell and the C2NLoS Urban Macro-Cell.

The MPS used in this work has 16 antennas and 8 delay taps. The delay taps are chosen from a fixed set of optical fibre delay lines, and can therefore not be tuned arbitrarily. These delays do not necessarily match those in the channel model. Furthermore, the fixed azimuth angles at which the MPS antennas are placed do not necessarily match the incidence angles in the channel model.

Because of these physical limitations, it is not feasible to implement the exact signal paths of the channel model in the MPS. Instead of using a truncation approach in which only the most significant signal paths of the channel model are considered [87], a new approach is proposed here.

We propose a method where focus is on matching the statistical parameters of the channel in the MPS, taking into account all signal paths of the channel model, and using all MPS antennas. The method for doing this is as follows: The channel model is characterized by a number of signal paths, each with a certain delay $(\tau)$, AoA $(\alpha)$, power $(P)$, and cross polarization ratio $(X P R)$. If we assume a number $N$ of signal paths, excess delay mean and spread are given by

$$
\begin{gathered}
\mu_{\tau}=\frac{\sum_{i=1}^{N} \tau_{i} P_{i}}{\sum_{i=1}^{N} P_{i}} \\
\sigma_{\tau}=\sqrt{\frac{\sum_{i=1}^{N}\left(\tau_{i}-\mu_{\tau}\right)^{2} P_{i}}{\sum_{i=1}^{N} P_{i}}}
\end{gathered}
$$

and the AoA mean and spread are defined as 


$$
\begin{array}{r}
\mu_{\alpha}=\arg \left(\frac{\sum_{i=1}^{N} e^{j \alpha_{i}} P_{i}}{\sum_{i=1}^{N} P_{i}}\right) \\
\sigma_{\alpha}=\sqrt{\frac{\sum_{i=1}^{N} \arccos ^{2}\left(\cos \left(\alpha_{i}-\mu_{\alpha}\right)\right) P_{i}}{\sum_{i=1}^{N} P_{i}}}
\end{array}
$$

For multipath richness we propose a parameter defined as

$$
R=\frac{\left(\sum_{i=1}^{N} P_{i}\right)^{2}}{\sum_{i=1}^{N} P_{i}^{2}}
$$

and the $k$-factor is defined as

$$
K=\frac{P_{L O S}}{\sum_{i=1}^{N} P_{N L O S, i}}
$$

Each MPS antenna pair $(\mathrm{V} / \mathrm{H})$ is considered one signal path, where the two $\mathrm{V} / \mathrm{H}$ antennas are used to set the proper XPR. The approach is to distribute the delays and set the power levels over the antenna pairs so as to achieve the same $\sigma_{\tau}$ and $\sigma_{\alpha}$ as in the channel model. If possible, $\mu_{\alpha}$ should also be the same, but this is not important since the reference direction is arbitrary anyway. Given these constraints, for LoS channel models it is of interest to achieve the same $R$ and $k$-factor in the MPS as in the channel model. For NLoS models, $R$ in the MPS can usually not reach the same value as in the channel model, but it is still of interest to maximize it, whereas the $k$-factor is irrelevant.

Applying this method to implement the Winner II B1LoS and C2NLoS models, result in the settings seen in Table 11 and Table 12. Figs. 21 and 22 show measurements of the power delay profile with the respective models implemented.

After establishing the delay distribution and branch powers according to the above, the Doppler shifts on all MPS branches should be determined. This is done by assuming a direction of travel and velocity relative to the array. The Doppler shifts are given by

$$
f_{d, i}=\frac{v_{d u t}}{\lambda} \cos \left(\alpha_{d u t}-\alpha_{i}\right)
$$

where $\alpha_{d u t}$ is the simulated direction of travel and $v_{d u t}$ the simulated velocity. 
Measurement Techniques Enhancements for MIMO 4G Mobile Communication Systems.

Extension of Mode Stirred Reverberation Chambers (MSRCs) Emulation Capabilities

Table 11. "B1 LoS” Channel Model Parameters

\begin{tabular}{|c|c|c|}
\hline Antenna Pair & Delay (ns) & Power (dB) \\
\hline 1 & 405 & -32.7 \\
\hline 2 & 4870 & $-\operatorname{lnf}$ \\
\hline 3 & 695 & -27.6 \\
\hline 4 & 1065 & $-\operatorname{lnf}$ \\
\hline 5 & 55 & 0 \\
\hline 6 & 0 & -5.3 \\
\hline 7 & 122 & -9.0 \\
\hline 8 & 2245 & $-\operatorname{lnf}$ \\
\hline $\begin{array}{c}\text { Excess Delay } \\
\text { Spread (ns) }\end{array}$ & \multicolumn{2}{|r|}{36} \\
\hline AoA Mean (Deg) & \multicolumn{2}{|r|}{4} \\
\hline AoA Spread (Deg) & \multicolumn{2}{|r|}{29} \\
\hline Richness & \multicolumn{2}{|r|}{1.8} \\
\hline K-factor (dB) & \multicolumn{2}{|c|}{3.3} \\
\hline XPR (dB) & \multicolumn{2}{|r|}{9} \\
\hline
\end{tabular}

Table 12. “C2 NloS” Channel Model Parameters

\begin{tabular}{|c|c|c|}
\hline Antenna Pair & Delay (ns) & Power (dB) \\
\hline 1 & 405 & -32.7 \\
\hline 2 & 4870 & $-\operatorname{lnf}$ \\
\hline 3 & 695 & -27.6 \\
\hline 4 & 2245 & $-\operatorname{lnf}$ \\
\hline 5 & 122 & 0 \\
\hline 6 & 0 & -5.3 \\
\hline 7 & 55 & -9.0 \\
\hline 8 & 1065 & $-\operatorname{lnf}$ \\
\hline $\begin{array}{c}\text { Excess Delay } \\
\text { Spread (ns) }\end{array}$ & & 235 \\
\hline AoA Mean (Deg) & & -13 \\
\hline AoA Spread (Deg) & & 49 \\
\hline Richness & & 14.2 \\
\hline K-factor (dB) & & Not applicable \\
\hline XPR (dB) & & 7 \\
\hline
\end{tabular}




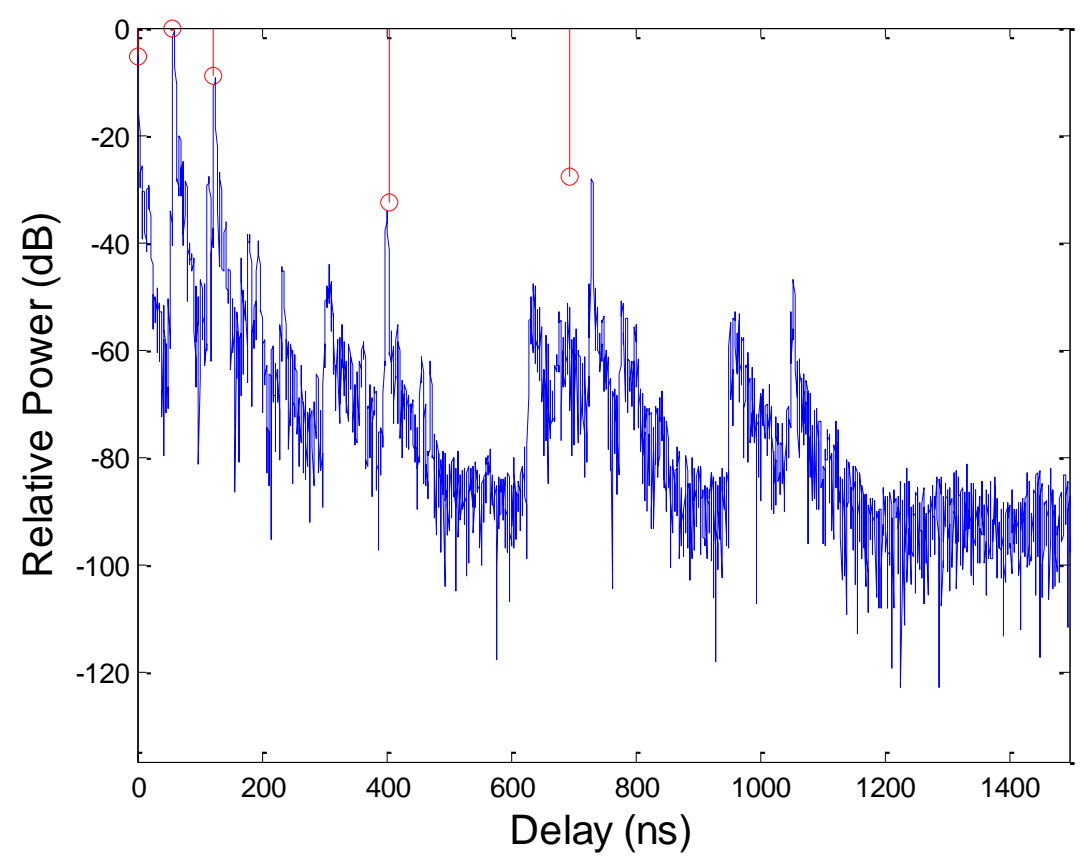

Figure 21. Power delay profile measured in the MPS for the scenario "B1 LoS". In red the ideal taps of the scenario.

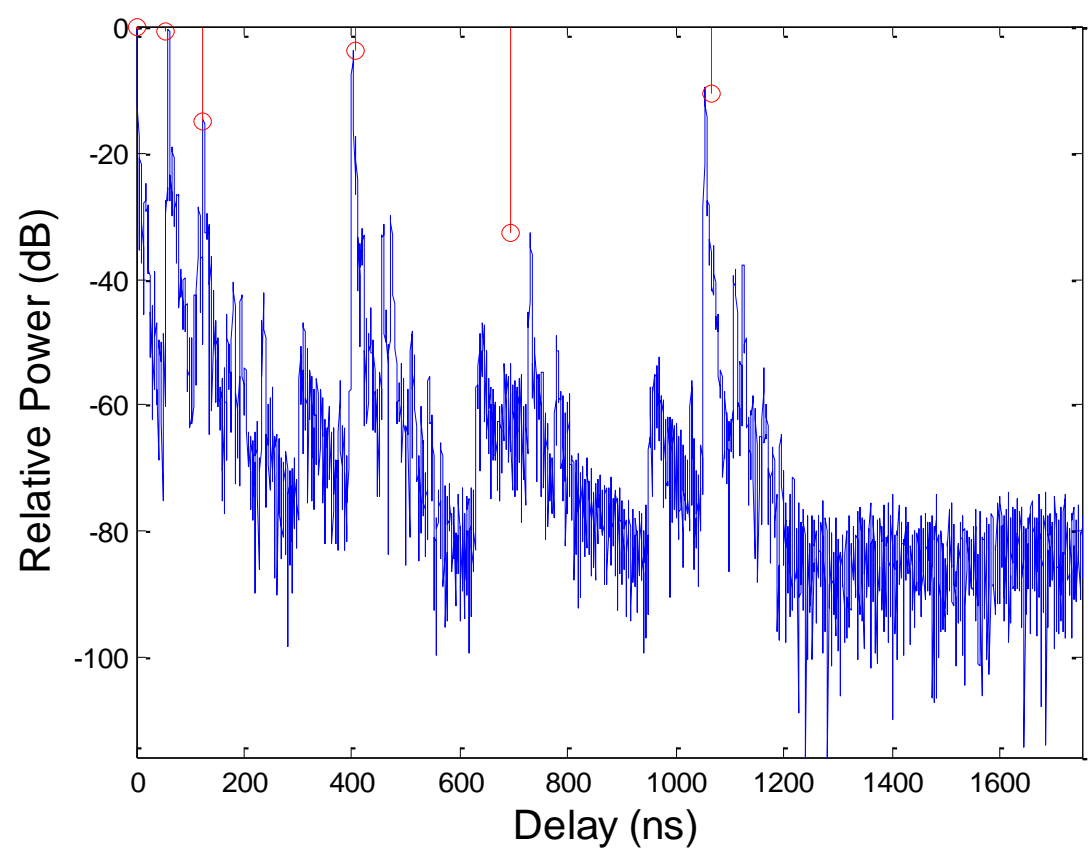

Figure 22. Power delay profile measured in the MPS for the scenario "C2 NLoS". In red the ideal taps of the scenario.

\subsubsection{User Influence Analysis}

HSDPA throughput is measured under the two different channel models. Measurements are taken with the phone oriented in three different planes inside the MPS, in order to take into account the antenna radiation pattern [88]. The three planes are referred as $X Y, Y Z$ and $X Z$, as described in Fig. 23. The phone is rotated over 12 
different positions in steps of 30 degrees for each plane. The antennas of the MPS define a ring in the $X Y$ plane, cf. Fig. 23.

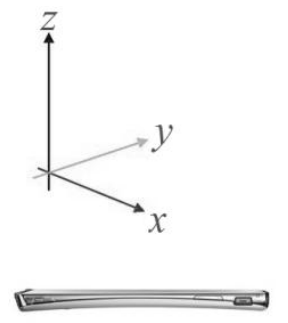

(a)

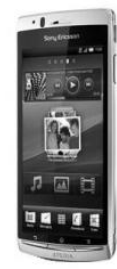

(b)

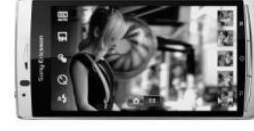

(c)

Figure 23. Different phone's orientations used during measurements without user influence.

In Fig. 24 is shown the throughput comparison for the two different channel models. Each colour denotes each one of the three planes defined for each phone. Slight differences can be seen between the two channel models, but not a clear tendency when we look at the average throughput.

Figs. 25 and 26 show plots of throughput performance for all the measurement repetitions when the phone is under rotation. Different colour stands for different orientation of the phone inside the MPS. Here we can see a big difference between the results obtained under each of the channel models. The results for the model with LoS show a big spread of the throughput performance for different positions of the phone. For the case of NLoS, the spread is clearly lower between measurements.

Detailed standard deviation of the measurements is shown in Fig. 27 for the different cases. There we can see how standard deviation of the measurement with the B1LoS channel model is almost twice the deviation seen in the other channel model. This effect is very similar for all the orientations of the phone under study. 


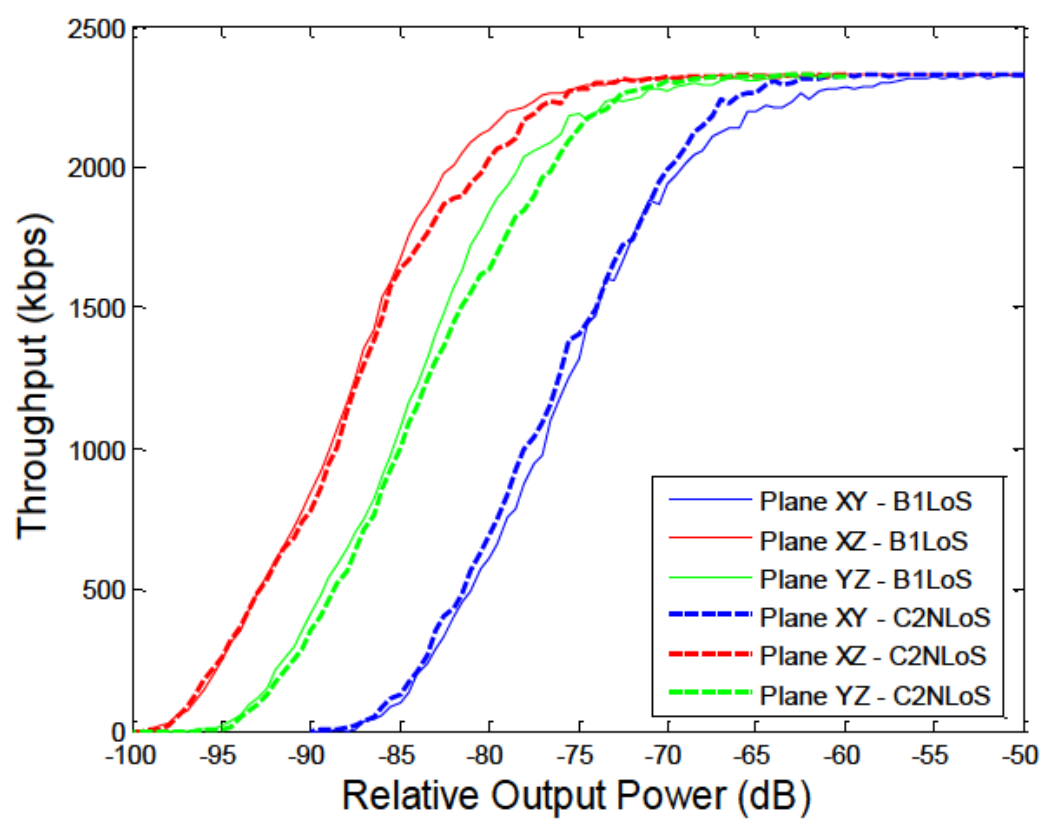

Figure 24. Throughput average for all combinations of channel model and phone orientation, without user influence.

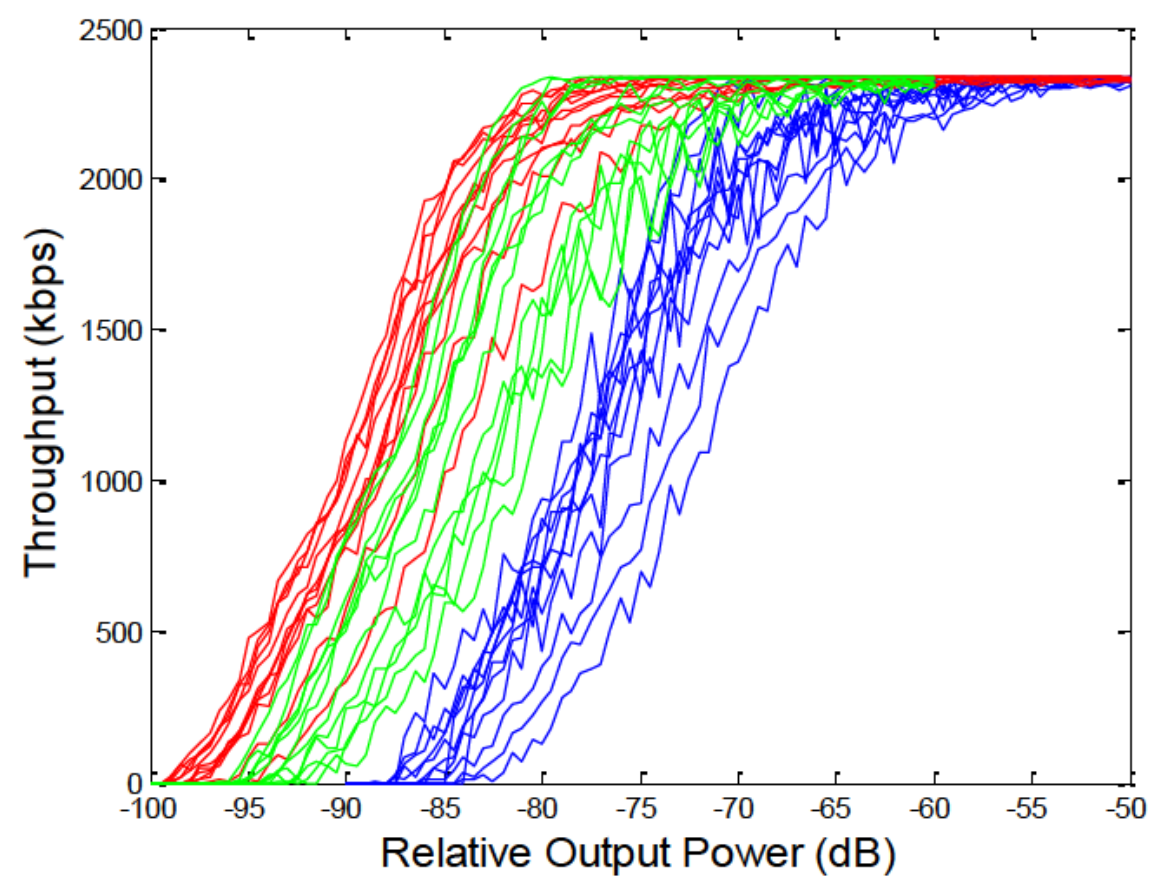

Figure 25. Throughput results for all the positions measured with channel model "B1 LoS". Different colors define the three orientation planes for the phone. 


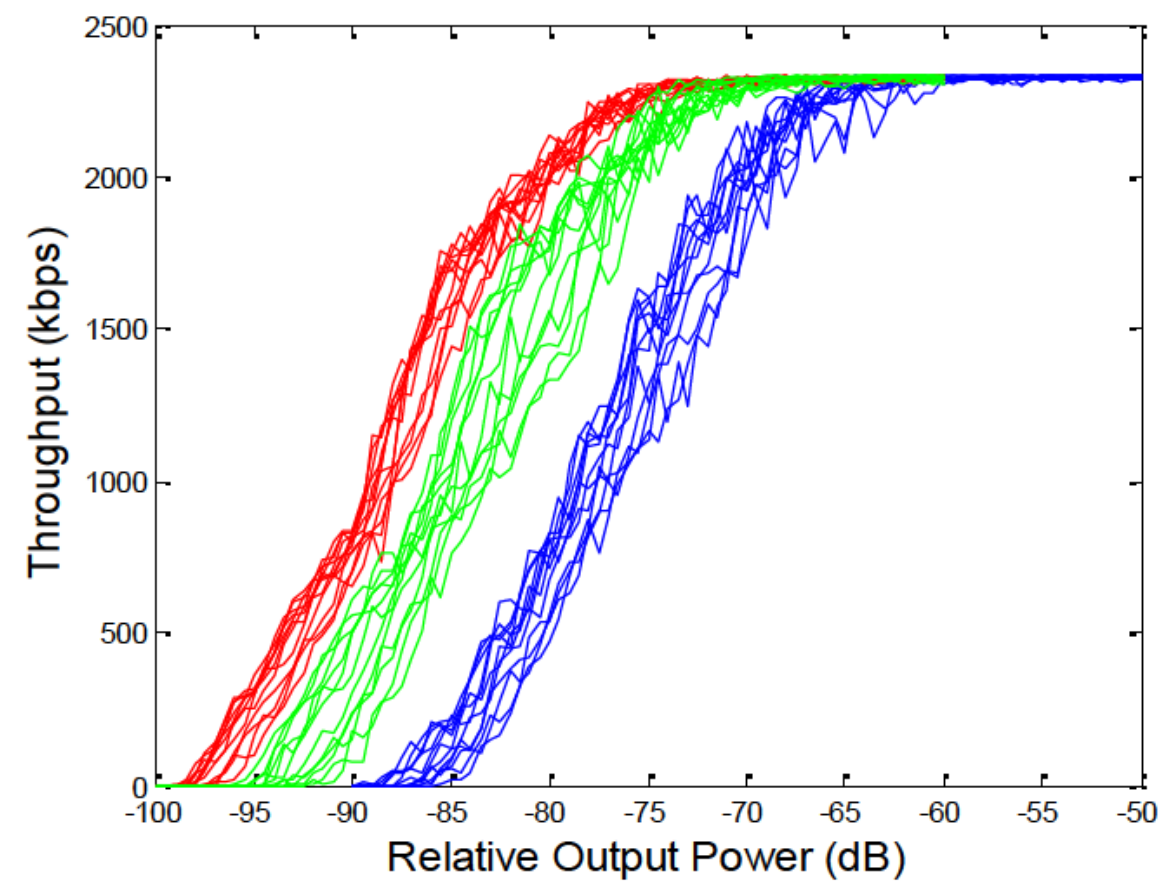

Figure 26. Throughput results for all the positions measured with channel model "C2 NLoS". Different colors define the three orientation planes for the phone.

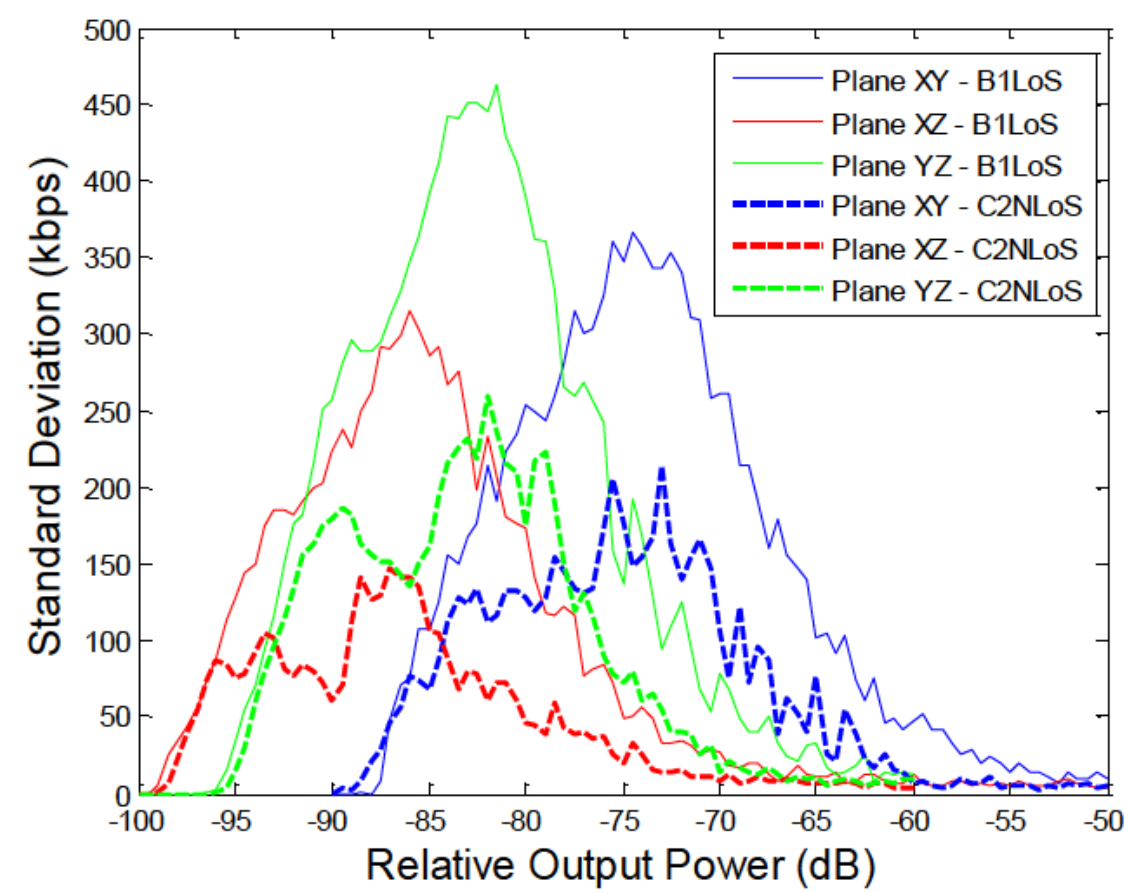

Figure 27. Standard deviation of the throughput performance for the two channel models under study. Different colors define the three orientation planes for the phone.

Now, it will be studied the influence of the user for each of the proposed channel models. Three different cases are studied; a) no user, b) head \& hand phantom and c) real person. 
For the no user case the measurements are the same as in the previous section, taking only the measurements for the vertical phone orientation (plane $\mathrm{YZ}$, as defined in Figure 23). This approximation is used because that orientation is the more similar to the orientation that the phone has when measurements are performed with user influence.

Case b) is implemented placing a head phantom inside the MPS and using also a hand phantom to hold the phone. Case $\mathrm{c}$ ) is implemented placing a real person inside the MPS, holding the phone with the right hand, tight to the right cheek. Results are shown in Fig 28.

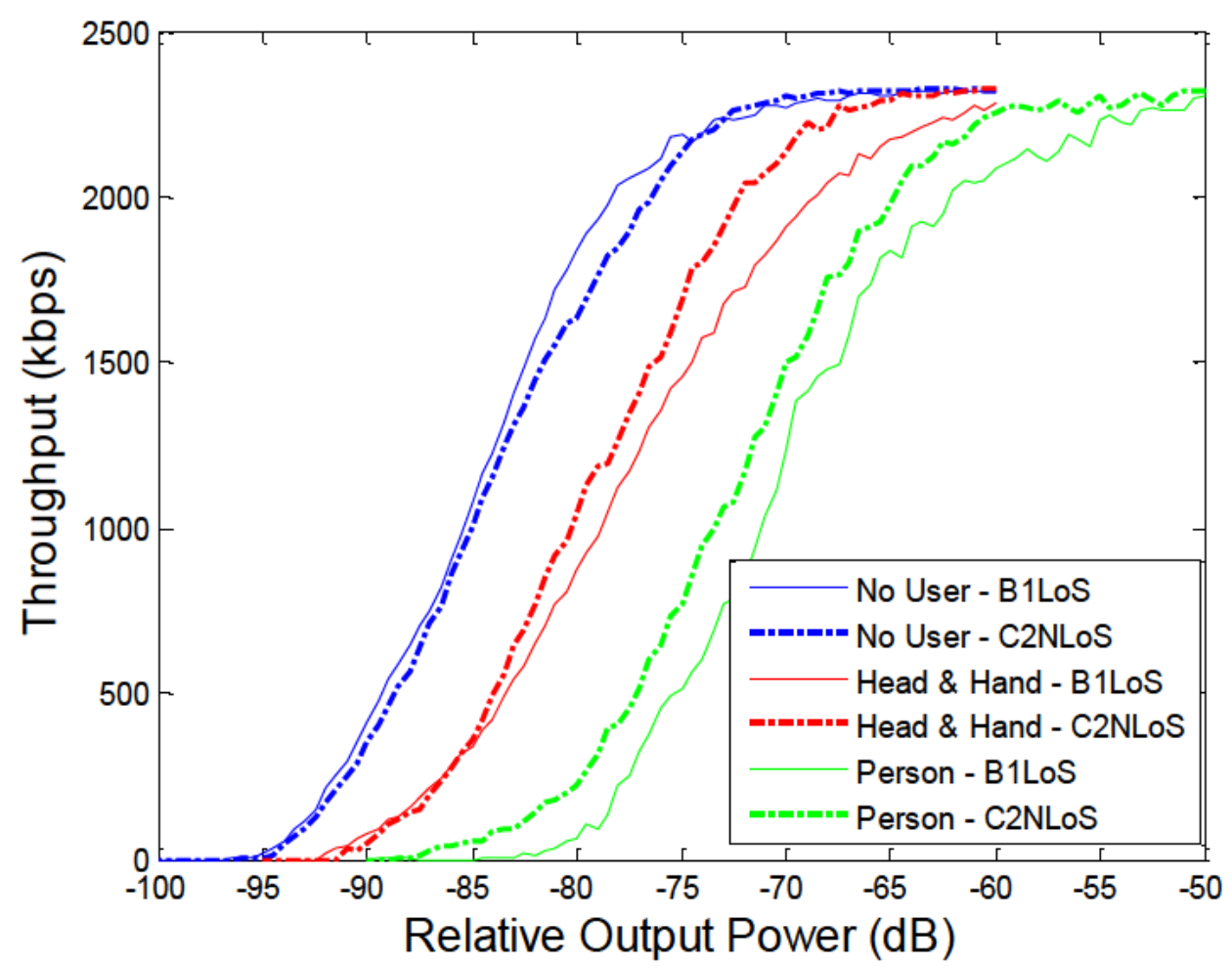

Figure 28. Throughput results for all the user effect cases with the two different channel models. Different colors define the three user effect cases.

It is clearly shown that the effect of the user presence introduces important losses. But it is even more interesting to see how the user affects the way the phone performance is degraded under power reduction. In order to see that effect, we take from Fig. 28 the power values where the throughput performance reaches $90 \%$ and 10 $\%$ of the maximum. The point where the phone reaches $90 \%$ of throughput (or $10 \%$ of BLER), is called "Reference Sensitivity Level" and is defined in [89] for HSDPA devices.

With the values extracted from Fig. 28 we can see if the user effect is just a loss component or if it also affects the curve shape. Results are shown in Table 13. 
TABLE 13. SensitiVITY LeVELS

\begin{tabular}{|c|c|c|c|c|}
\hline $\begin{array}{c}\text { Channel } \\
\text { Model }\end{array}$ & User Case & $\begin{array}{c}90 \% \\
\text { Throughput } \\
\text { Power Level } \\
\text { (dB) } \\
\end{array}$ & $\begin{array}{c}10 \% \\
\text { Throughput } \\
\text { Power Level } \\
\text { (dB) } \\
\end{array}$ & $\Delta(\mathrm{dB})$ \\
\hline B1 LOS & $\begin{array}{c}\text { No User } \\
\text { Head \& Hand } \\
\text { Phantom } \\
\text { Real Person }\end{array}$ & $\begin{array}{l}-76.336 \\
-66.736 \\
-59.665\end{array}$ & $\begin{array}{l}-91.731 \\
-86.709 \\
-77.857\end{array}$ & $\begin{array}{l}15.395 \\
19.973 \\
18.192\end{array}$ \\
\hline C2 NLOS & $\begin{array}{c}\text { No User } \\
\text { Head \& Hand } \\
\text { Phantom } \\
\text { Real Person }\end{array}$ & $\begin{array}{l}-75.455 \\
-70.582 \\
-62.955\end{array}$ & $\begin{array}{l}-91.205 \\
-86.540 \\
-78.893\end{array}$ & $\begin{array}{l}15.75 \\
15.95 \\
15.93\end{array}$ \\
\hline
\end{tabular}

If we look at the results, we can see how the channel models affect throughput differently for the no user case and the cases with user presence. In the no user case, the performance of the phone under the B1LoS is slightly better for the $90 \%$ level. For the cases with user influence instead, the phone shows better performance under the channel model with NLoS.

These results can be explained, since the angle spread of the incoming waves is significantly lower for the LoS channel model, so the effect of the user shadowing is much more important. In the no user case, the incoming signal is mostly confined in one direction, so the delay spread of the channel model is lower (as showed in Tables 12 and 13), and the performance is slightly better. This behaviour seems to agree with other studies that compare the same metric with others OTA techniques [90] [91] [92] [93].

Another important difference between the channel models is seen if we look at the curve shape. For the NLOS channel model, the curve shapes of the three cases under study are very similar, being only a power offset between them due to the path losses introduced by the user. We can see in the last column of Table 14 that the difference between the $90 \%$ and $10 \%$ level is just a few tenths of $\mathrm{dB}$. On the other hand, for the LoS channel model, the curve shape is affected by the user presence, and the difference between $90 \%$ and $10 \%$ levels differs more than $4.5 \mathrm{~dB}$ from the no user case to the others user influenced cases.

\subsection{Conclusion}

In this section we have evaluated the user influence over correlation and diversity gain of some antenna prototypes, as well as HSDPA throughput for a commercial mobile phone for some different scenarios including a real person. 
Passive antenna results show that user influence equalizes MIMO performance of devices which have very different behaviour when analysed without user influence. Furthermore, the user influence on correlation does not seem to be a linear constant offset but a spread effect.

This calls for detailed studies with different body phantoms in order to define the effect of the user influence over the antenna, and distinguish it from the shadowing effect that actually changes the propagation environment where the antenna is being measured. Further research includes the development of a theoretical model for the user influence on MIMO devices.

Regarding the active performance of a commercial phone, several measurements are presented with and without user influence. It is demonstrated that throughput performance of a device depends upon the user influence and the propagation channel model in a complex way.

Some conclusions can be drawn from the results, which seem to relate the channel model parameters with the final performance of the device. Looking at the reference sensitivity levels of the no user case, we observed that there is little difference between the two of the channel models measured in the MPS. In fact, if we look at the sensitivity point of the channel B1LoS measured in the MPS (with a DS of 36 ns), we see that it is slightly better than the values obtained under the C2NLoS scenario (with a DS of $235 \mathrm{~ns}$ ). Therefore, results without user influence seem to show a dependency of the performance of the device on the delay spread of the simulated channel.

On the other hand, user influence under different channel models seems to be more complex, and no clear pattern is seen. This highlights the importance of measuring with user presence, as the effect of the user is different under different propagation environments. 


\section{Study on the Effect of a Limited Number of Signal Paths Over Wireless Devices Performance}

\subsection{Influence Analysis over Passive Antenna Parameters}

The benefits of MIMO strategies rely on the assumption that a large number of signal paths is present at the user position, so there exists a rich environment around the receiving antennas [94] [95]. As an example, when antenna correlation is calculated from the radiation pattern of both antennas, it is necessary to perform an integral which is in fact an infinite summation of signal paths. However there is not in that integral any information about the simultaneous number of signal paths at a given moment.

Furthermore, most of the typical channel models used in industry assume a large number of incident waves at the DUT position. This assumption is fair for many real cases, especially when the user is in an urban environment. However, for other reallife environments this assumption is debatable, and some studies show that sometimes most of the transferred power is contained in only a few signal paths [96] [97] [98] [99] [100].

Emulation of multipath environments can be easily performed using MSRCs [48], which have become one of the established techniques to do OTA measurements during the last few years. However, it is interesting to characterize devices in environments without a large number of components.

This analysis can be easily done using the same MPS used in the previous chapter, since it has attenuators in each signal path, which allows switching on and off each of the antennas via software. Using that feature of the MPS, it is possible to change the number of paths composing the link, without changing the DUT position. Each signal path in the MPS has a phase shifter in order to emulate the fast fading environment experienced in a multipath environment by any device under real use conditions. 
In this section, it is presented a study to characterize the effect that the number of signal paths has over the characterization of dual antennas. The purpose is to have experimental indications of how performance typically differs depending on the number of signal paths, and thereby also indications regarding the need to consider the number of signal paths in characterization and therefore in channel models definition.

A 4-port VNA is used for this study so it is possible to measure the complex transmission coefficients $S_{21}$ between the MPS antennas and each of the two DUT antenna ports of the test object simultaneously. Fig. 29 shows a block diagram of the setup,

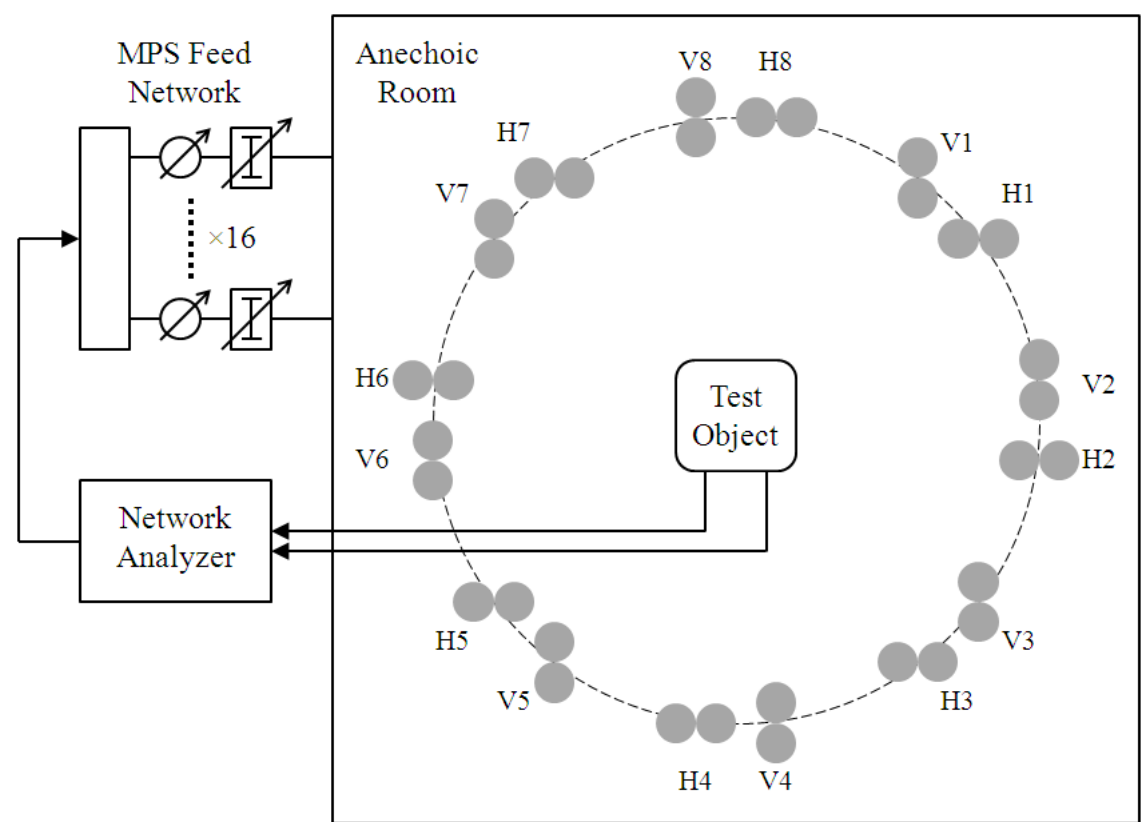

Figure 29. Block diagram of the setup for S-parameter measurements on dual antennas.

Two $S_{21}$ sequences of 6401 time values are taken for each prototype, which last 20 seconds approximately. Therefore, the maximum Doppler shift set in the MPS phase shifters to generate fast fading is $50 \mathrm{~Hz}$ so there is enough fading density along the $20 \mathrm{~s}$ to have a reliable measurement

Table 14 shows which MPS antennas are switched on for each measurement, according to the definition setup showed in Figure 29. Measurements are performed with 2, 4, 8, and 16 MPS antennas active, and the antennas that are switched on all have equal amplitude. Active antennas are marked in Table 16 with an "O". With 2, 4, and 8 paths, there are three different sets of active antennas, given by the three columns. 
Table 14. Phase sweep rate on the MPS branches, and the active branches used for the DIFFERENT NUMBER OF PATHS. FOR 2, 4, AND 8 PATHS, THREE SETS ARE USED, EACH GIVEN BY A COLUMN.

\begin{tabular}{|c|c|c|c|c|c|c|c|c|c|c|}
\hline $\begin{array}{c}\text { MPS } \\
\text { Branch }\end{array}$ & \multicolumn{3}{|c|}{$\begin{array}{c}2 \\
\text { Paths }\end{array}$} & \multicolumn{3}{|c|}{$\begin{array}{c}4 \\
\text { Paths }\end{array}$} & \multicolumn{3}{|c|}{$\begin{array}{c}8 \\
\text { Paths }\end{array}$} & $\begin{array}{c}16 \\
\text { Paths }\end{array}$ \\
\hline V1 & 0 & 0 & - & 0 & 0 & - & 0 & 0 & - & 0 \\
\hline H1 & - & - & - & - & - & - & - & 0 & - & 0 \\
\hline V2 & - & - & 0 & 0 & - & 0 & 0 & - & 0 & 0 \\
\hline $\mathrm{H} 2$ & - & - & - & - & - & - & - & - & 0 & 0 \\
\hline V3 & - & - & - & - & - & - & - & 0 & - & 0 \\
\hline $\mathrm{H} 3$ & - & - & - & - & 0 & - & 0 & 0 & - & 0 \\
\hline V4 & - & - & - & - & - & - & - & - & 0 & 0 \\
\hline $\mathrm{H} 4$ & 0 & - & - & - & - & 0 & 0 & - & 0 & 0 \\
\hline V5 & - & - & - & - & 0 & - & 0 & 0 & - & 0 \\
\hline H5 & - & 0 & - & 0 & - & - & - & 0 & - & 0 \\
\hline V6 & - & - & - & - & - & 0 & 0 & - & 0 & 0 \\
\hline H6 & - & - & 0 & 0 & - & - & - & - & 0 & 0 \\
\hline V7 & - & - & - & - & - & - & - & 0 & - & 0 \\
\hline $\mathrm{H} 7$ & - & - & - & - & 0 & - & 0 & 0 & - & 0 \\
\hline V8 & - & - & - & - & - & - & - & - & 0 & 0 \\
\hline $\mathrm{H} 8$ & - & - & - & - & - & 0 & 0 & - & 0 & 0 \\
\hline
\end{tabular}

Results are analyzed in terms of the correlation coefficient $\rho_{c}$ and ADG. Expressions used for the calculation are the same from those defined in Chapter 2 for MSRCs, but in this case the averages are taken over the time (instead of over a stirring sequence), since the fading condition is here generated by the phase shifters (instead of by the stirrers movement). Then, the used expressions are,

$$
\rho_{c}=\frac{\left\langle\left(S_{21,1}-\left\langle S_{21,1}\right\rangle\right)\left(S_{21,2}-\left\langle S_{21,2}\right\rangle\right)^{*}\right\rangle}{\sqrt{\left\langle\left|S_{21,1}-\left\langle S_{21,1}\right\rangle\right|^{2}\right\rangle\left\langle\left|S_{21,2}-\left\langle S_{21,2}\right\rangle\right|^{2}\right\rangle}}
$$

And

$$
\left\langle S_{21, x}\right\rangle=\frac{1}{6401} \sum_{t=1}^{6401} S_{21, x}(t)
$$

Where $S_{21,1}$ and $S_{21,2}$ are the complex transmission coefficients of the respective test object antenna, each being a sequence of 6401 samples.

DG is calculated according to the equations (49) and (50) using the MRC combining scheme. Again, two values are calculated taking each of the antennas as the reference, so there are no assumptions about the non-diversity case.

For this study the same test objects referred as Prototype A and Prototype B in the previous section are used. As it was said before, one of those test objects is designed 
on purpose to have a bad performance. These prototypes work in the $700 \mathrm{MHz}$ band, so measurements are taken at $740 \mathrm{MHz}$. The antennas are accessed via coaxial cables, which exit the test objects on the middle of the long side for minimum interference. In order to minimize the cables effect over the measurements accuracy as much as possible, the cables are routed so as to minimize influence on the antenna performance [101] and ferrites absorbers are attached to them [102].

Measurements are repeated in three orthogonal planes, with the DUT placed at the centre of the antenna ring over a dielectric mounting system. Therefore, the total number of measurements for each test object is $9 / 9 / 9 / 3$ for the respective 2/4/8/16 paths.

\subsubsection{Correlation}

Results are seen in Fig. 30, with circles for Prototype A and triangles for Prototype $B$. The plots show that also in this experiment, reducing the number of signal paths leads to a much greater spread in the different metrics. For both test objects, $\left|\rho_{c}\right|$ is clearly worse with 16 paths than in a 3-D environment with many paths, which is 0.35 for Prototype A and 0.56 for Prototype B (measured in the previous chapter).

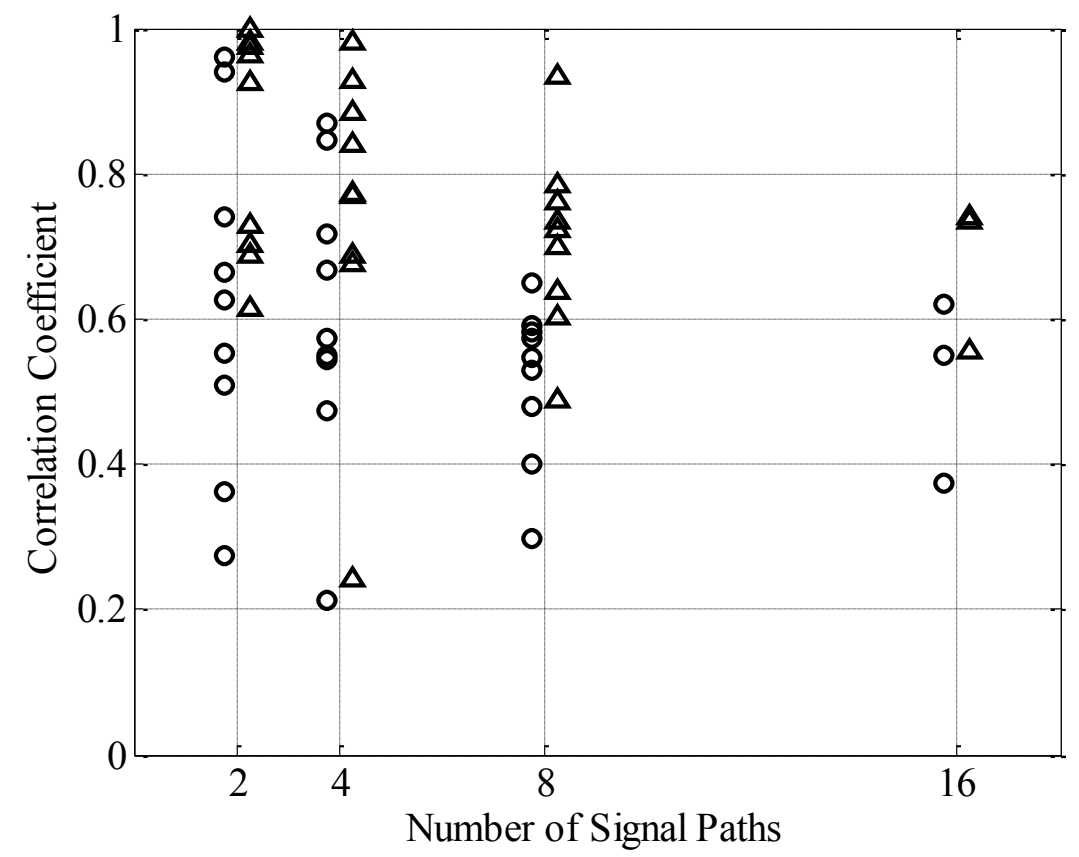

Figure 30. Correlation coefficient $\left|\rho_{c}\right|$ of Prototype A (circles) and Prototype B (triangles), as a function of the number of signal paths. 
Table 15. Proportion of Correlation coefficient Values less than 0.7 for Prototypes A and $B$, FOR DIFFERENT NUMBER OF SIGNAL PATHS.

\begin{tabular}{|c|l|c|c|c|c|}
\hline \hline Prot. & Parameter & 2 Paths & 4 Paths & 8 Paths & 16 Paths \\
\hline A & Proportion of $\left|\rho_{c}\right|<0.7$ & $67 \%$ & $67 \%$ & $100 \%$ & $100 \%$ \\
B & Proportion of $\left|\rho_{c}\right|<0.7$ & $22 \%$ & $33 \%$ & $44 \%$ & $33 \%$ \\
\hline \hline
\end{tabular}

At 16 signal paths, $\left|\rho_{c}\right|$ is higher for Prototype B than Prototype $A$, and it is significantly higher with fewer signal paths. This is contradictory with the results obtained in the MSRC, with a large amount of signal paths. It is common to accept a correlation value of 0.7 as the border line to distinguish between a device with good and bad diversity performance.

Table 15 shows the proportion of $\left|\rho_{c}\right|$ values less than 0.7 for the two prototypes, indicating that Prototype $A$ is superior. However, the spread is very large for all the cases, and especially dramatic for the 2 signal measurements.

\subsubsection{DG}

DG results for both prototypes is shown in Fig. 31 and Table 16.

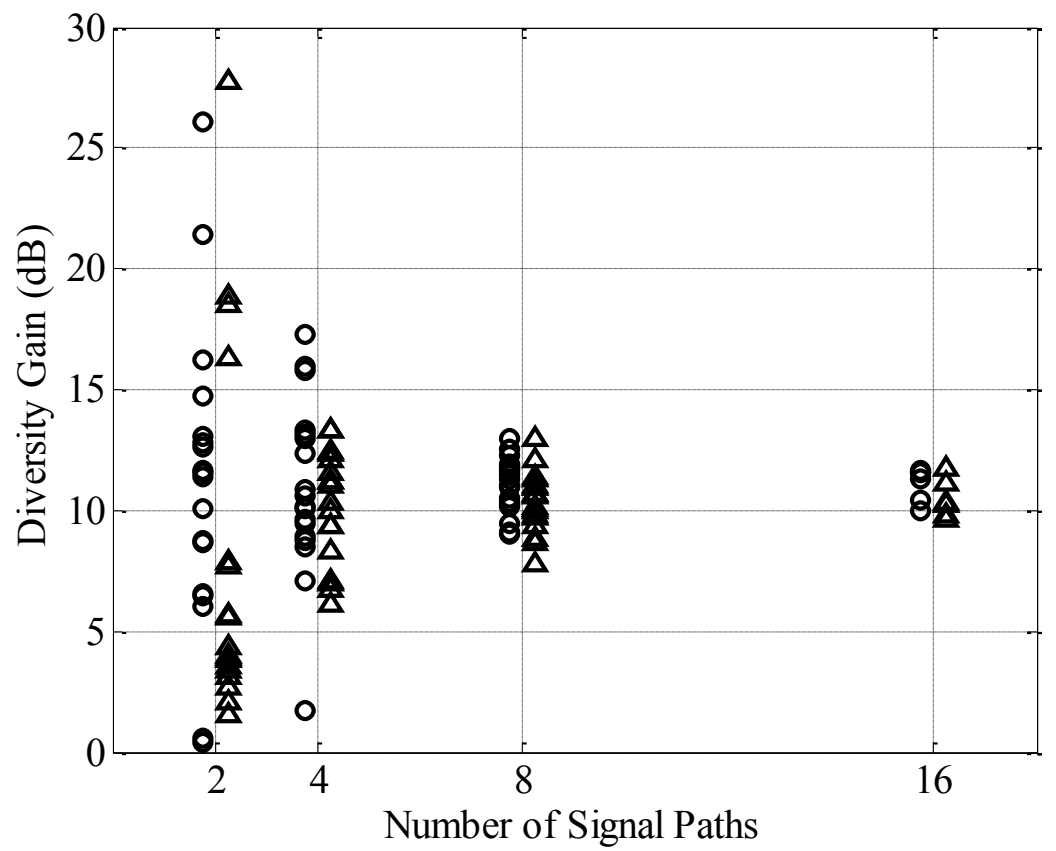

Figure 31. Diversity gain of Prototype A (circles) and Prototype B (triangles), using maximum ratio combining, as a function of the number of signal paths. 


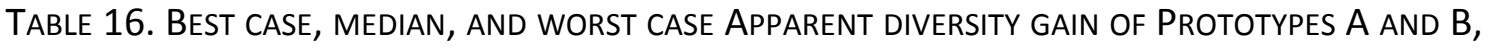
FOR DIFFERENT NUMBER OF SIGNAL PATHS.

\begin{tabular}{clcccc}
\hline \hline Prot. & Parameter & 2 Paths & 4 Paths & 8 Paths & 16 Paths \\
\hline A & Best case $G_{d}(\mathrm{~dB})$ & 26.1 & 17.3 & 13.0 & 11.6 \\
B & Best case $G_{d}(\mathrm{~dB})$ & 27.8 & 13.3 & 12.9 & 11.7 \\
A & Median $G_{d}(\mathrm{~dB})$ & 11.5 & 10.4 & 11.1 & 11.4 \\
B & Median $G_{d}(\mathrm{~dB})$ & 4.2 & 10.7 & 10.4 & 10.3 \\
A & Worst case $G_{d}(\mathrm{~dB})$ & 0.4 & 1.7 & 9.0 & 10.0 \\
B & Worst case $G_{d}(\mathrm{~dB})$ & 1.6 & 6.1 & 7.8 & 9.6 \\
\hline \hline
\end{tabular}

It is observed that the median values remain quite stable when the number of signal paths is decreased (except for the case of Prototype B with 2 paths), and those values are similar to the actual DG measures in a MSRC in the previous Chapter (which are $8.92 \mathrm{~dB}$ and $10.34 \mathrm{~dB}$ for Prototype $A$ and $B$ respectively). However, the fluctuation in the minimum and maximum values shows a very important spread in the measurements. Therefore, when the number of paths becomes very low it is not possible to state that one device is better than the other, since there is a very large variation in the performance (more than $27 \mathrm{~dB}$ of difference between the best and the worst case).

\subsection{Influence Analysis over Active Wireless Devices}

\subsubsection{HSDPA Data Throughput}

In this section it will be analysed the effect that a reduction in the number of signal paths has over the en-to-end data throughput of a HSDPA commercial device. Taking over the work published in [103] for passive antennas, this study presents measurements of HSDPA throughput using an MPS with 2-8 paths. The results are analyzed in terms of average and standard deviation throughput, as well as sensitivity levels.

The MPS used in this study consist of an array of 8 dual-polarized antennas (16 antennas in total) distributed in a ring of $1.4 \mathrm{~m}$ radius. Each of the 8 dual antennas is fed through a fibre optic delay line which introduces a fixed delay, different for each dual antenna. During this study we only use the dual antennas fed through the 4 fibre optics with the closest lengths, in order to avoid as much as possible the effect of delays on the study. Lengths of the used fibres are 11, 24.5, 81 and 139 meters. 
Results are analyzed in terms of throughput. H-Set 3 reference channel is used to perform this study, according to the definition in table C.8.1.3 of [6]. 16-QAM modulation and HSDPA Level Set 3 are used (Table E.5.8 of [6]). Other testing conditions are extracted from [8].

A Rohde \& Schwarz CMU-200 BSE is used as measurement equipment. Measurements are performed by doing a sweep over the output power level of the $\mathrm{BSE}$, and taking the throughput measurement for each power value. Each of these throughput samples are measured using 2400 HSDPA subframes, in about 15 seconds. A maximum Doppler shift of $50 \mathrm{~Hz}$ is used in the phase shifters, to ensure that 15 seconds allow enough fading during the measurement. Measurements are performed using a commercial phone HSDPA category 8 capable. Channel 10562 of HSDPA Band I is used $(2110 \mathrm{MHz})$. Three different cases are studied, with 2,4 , and 8 signal paths.

The MPS comprises 16 antennas, so there are different combinations of antennas that can be used to perform this study. Twelve realizations are taken for each case, using randomly chosen combinations of the antennas. The power is the same in each path in all measurements, so different number of paths lead to a different power level at the DUT. This effect is taken into account during the calibration of the MPS, so the measurement equipment (BSE) is properly configured with the correct link losses. Therefore, differences in performance are not expected to be due to changes in power level.

The DUT is placed at the centre of the MPS antenna array, and in free-space condition (without head or hand). Measurements are performed with the phone in vertical position, with respect to the plane defined by the antenna array. The antenna radiation pattern in that plane is close to omnidirectional, so the effect of radiation pattern changes is minimized. This effect cannot be completely avoided when using commercial phones.

Figure 32 is a box-and-whisker plot of the power levels where $90 \%$ of maximum throughput is reached, as a function of the number of signal paths. This $90 \%$ level is commonly used in industry as the sensitivity level of the phone [89]. Figure 33 shows the results for the power level where $10 \%$ of maximum throughput is reached. 


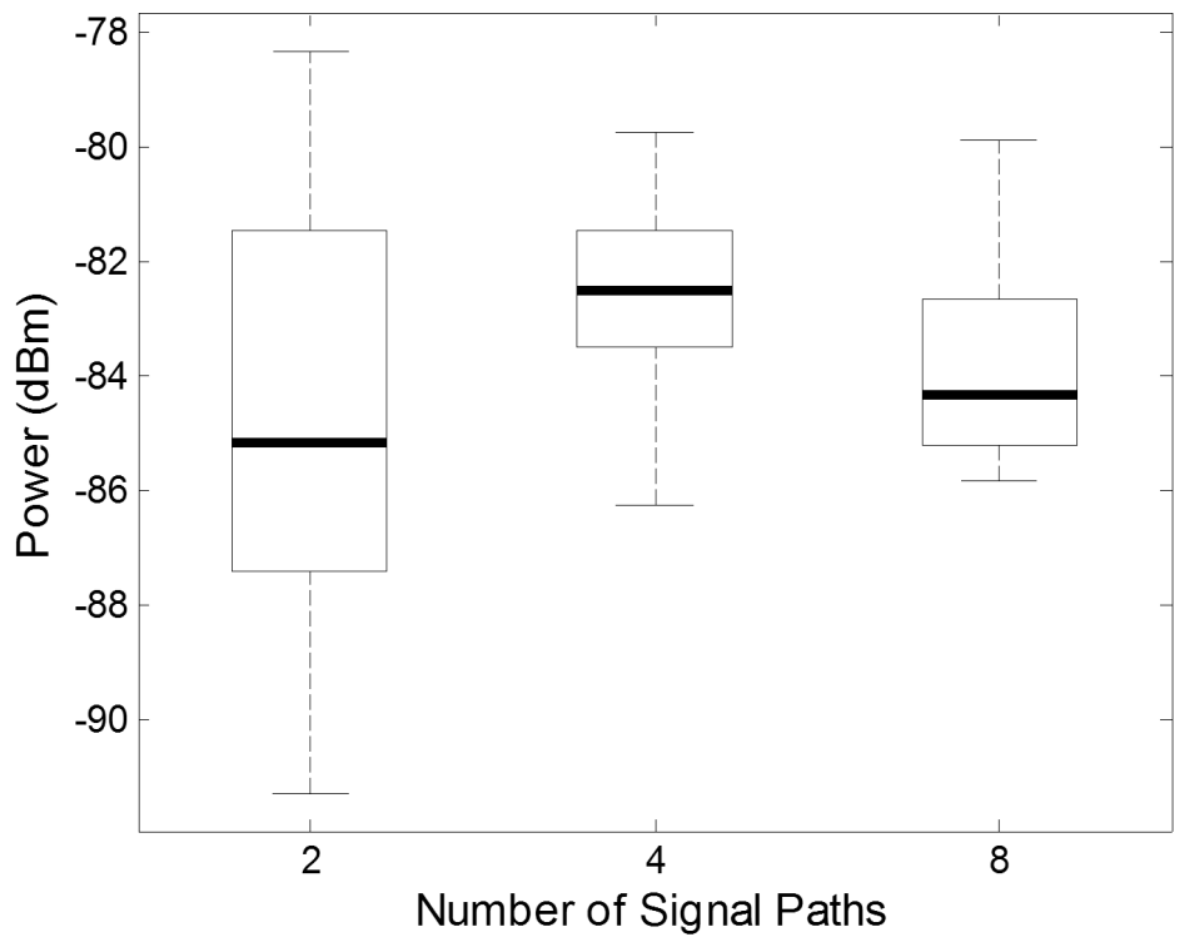

Figure 32. Power level where $90 \%$ of maximum throughput is reached, as a function of the number of signal paths. Boxes have lines at the lower quartile, median (bold line), and upper quartile values. Whiskers show the extent of the rest of the data.

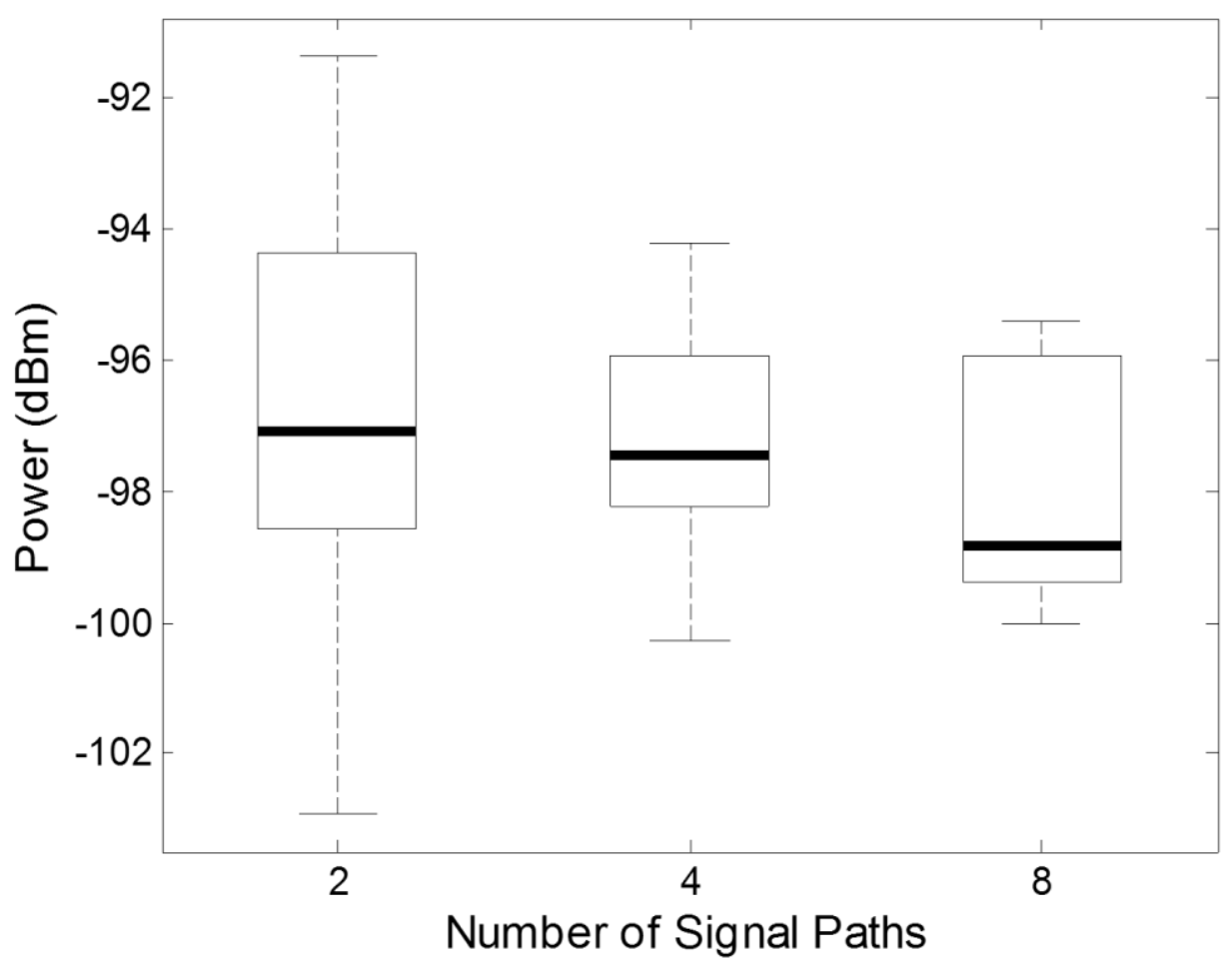

Figure 33. Power level where $\mathbf{1 0} \%$ of maximum throughput is reached, as a function of the number of signal paths. Boxes have lines at the lower quartile, median (bold line), and upper quartile values. Whiskers show the extent of the rest of the data. 
Both the $90 \%$ and the $10 \%$ levels show an important increase in the spread when the number of signal paths is reduced.

Differences are especially clear if we compare the case with 2 signal paths with any of the other cases. The spread with 2 paths is almost twice the spread of the 8 signal paths case. Even four signal paths show a big difference compared to two. Measurements are done under fading condition, so there is a spread in the measurement due to the fading. As mentioned before, measurements are taken over long enough time to ensure that the spread increase due to fading is similar in all cases.

Another effect observed is the different trend of mean and median power values between the $90 \%$ and $10 \%$ power levels. In Figure 32 it can be appreciated how the trend of the median power values is higher (worst performance) when the number of paths is increased. The effect is the opposite for the case of $10 \%$ throughput level. In that case an increase in the number of signal paths leads to lower mean values (better performance).

TABLE 16. Best CASE, MEDIAN, MEAN, AND WORST CASE AT $90 \%$ THROUGHPUt LEVEL, FOR DIFFERENT NUMBER OF SIGNAL PATHS

\begin{tabular}{l|c|c|c}
\hline \hline Parameter & 2 Paths & 4 Paths & 8 Paths \\
\hline Best case 90\% Level (dBm) & -91.3 & -86.2 & -85.9 \\
Median 90 \% Level (dBm) & -85.1 & -82.5 & -84.4 \\
Worst case 90 \% Level (dBm) & -78.5 & -79.7 & -79.9 \\
\hline \hline
\end{tabular}

TABLE 17. Best CASE, MEDIAN, MEAN, AND WORST CASE AT $10 \%$ THROUGHPUt LEVEL, FOR DIFFERENT NUMBER OF SIGNAL PATHS.

\begin{tabular}{l|c|c|c}
\hline \hline Parameter & 2 Paths & 4 Paths & 8 Paths \\
\hline Best case 10 \% Level (dBm) & -102.9 & -100.2 & -99.9 \\
Median 10 \% Level (dBm) & -97.1 & -97.4 & -98.9 \\
Worst case 10 \% Level (dBm) & -91.3 & -94.2 & -95.3 \\
\hline \hline
\end{tabular}


This effect can be explained if we take into account the fact that the HSDPA standard implements a scheme of retransmissions called "redundancy and constellation version coding" [104] which provides an incremental redundancy between retransmissions. When the phone is working at high power (around the maximum throughput, cf. Fig. 32), no retransmissions are needed, so the phone is more sensitive to the signal fading. On the contrary, when the phone works at low power (cf. Fig. 33), redundancy is added in the retransmissions, making the phone less sensitive to the signal fading. The signal fading is increased by a greater number of paths. Consequently performance is reduced at the $90 \%$ level but improved at the 10 $\%$ level, for larger number of signal paths.

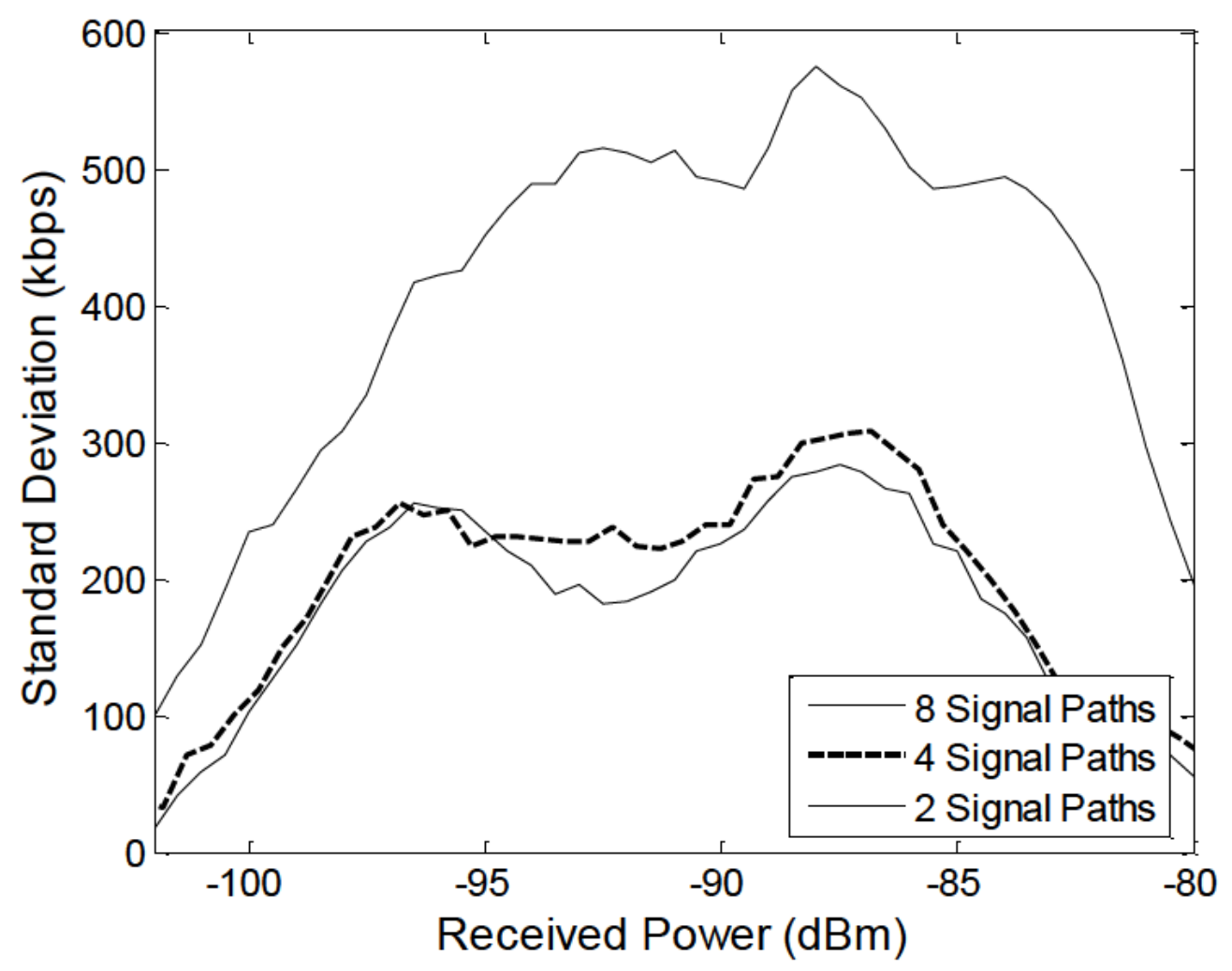

Figure 34. HSDPA throughput standard deviation as a function of received power.

In Fig. 34 is shown the standard deviation of the measurement for the three cases. Standard deviation is quite similar for the cases with 4 and 8 signal paths, but there is an important increase in the case with 2 signal paths. It is also seen that standard deviation of HSDPA throughput has an important dependency on the power level.

\subsection{Conclusion}

In this chapter, some dual antenna prototypes and a commercial mobile phone have been characterized using different number of signal paths, in order to characterize whether or not that metric is important for device characterization. 
Antenna correlation, diversity gain and HSDPA throughput are used to perform that analysis.

Several conclusions are drawn from the results. For the passive antennas, the performance with 16 signal paths shows only small variations over different test object orientations for all the analyzed metrics. However, when the number of signal paths is reduced, it is observed a large increase in the spread of all the metrics, what creates uncertainty in differentiating a good device and a bad one. Some degradation in average performance is also seen. For example, with two signal paths, antenna correlation can take almost any value with most of them being above the 0.7 level, even for a prototype with good performance in a rich environment. This shows the effect that a real environment with just a few signal paths can have over the device performance experienced by a telecommunications network user. This effect is appreciated in two test objects of the same type of terminal, but with different antenna solutions, but there also seems to be some differences in terms of median and worst case performance. Therefore, it is not possible to clearly state which antenna is better, since there is a dependency on the number of signal paths used for the characterization. Presented experiments show that characterization in environments with a limited number of signal paths provides a deeper insight into dual antenna performance compared to the traditional characterization assuming a large number of signal paths.

The main effect observed in the case of the commercial phone is mainly the same, since a reduction of the number of signal paths implies an increase in the dispersion of the measured values. With only two signal paths, differences of more than $10 \mathrm{~dB}$ in the sensitivity level of the phone are shown. Again, these big differences, can lead to a mistake differentiating a good and a bad phone.

Apart from that, in the commercial phone analysis some differences on the median values are found for different working zones of the DUT. This shows that the number of signal paths has a different effect when the phone is working around the maximum level of throughput ( $90 \%$ level) and when the phone works close to the minimum level (10\% level) due the implementation by the HSDPA standard of some redundancy between retransmissions [104].

Measurements presented in this section show that the number of signal paths in the channel models used for characterization is important. Most of the already defined channel models assume a large number of signal paths, but some realistic situations could require of characterization with a reduced number of signal paths. This characterization can be used to estimate the maximum variations in performance that a user can experience in a real environment with a limited number of signal paths. 


\section{Software Based Extension of MSRC Emulation Capabilities}

\subsection{Introduction}

In Chapter 3 of this thesis, the main characteristics that define the behaviour of an MSRC are presented, as well as some of the advances that have been proposed in recent years to improve the measurement capabilities of these. As it has been shown, the fading scenario typically generated in a MSRC follows a Rayleigh amplitude distribution, which is the typical environment found in urban scenarios. Yet, real propagating scenarios rarely follow an ideal Rayleigh-fading environment. The $k$-factor of more general Rician-fading environments changes as the distance of receiver to transmitter changes [105]. Macrocells usually offer a greater $k$-factor than microcells [106] while rich multipath environments provide $k$-factors typically close to zero [107]. Even more so, some others fading distributions as Nakagami or Log-Normal, have been detected to accurate emulate the fading scenarios found in some specific cases [67] (UWB channels, On-Body networks...). Consequently, recent research efforts have concentrated on extending the original capabilities of first-generation MSRCs. Good examples are the recent extension to non-isotropic environments [22] and the emulation of the effects of metallic windows and other artefacts, trees and walls in buildings [54], although this last one only for Rayleigh-fading scenarios.

However, these proposed amendments are based on hardware modifications of the MSRC original setup, such as the introduction of directive antennas and/or absorbers inside the cavity. These techniques, although potentially interesting, are difficult to control because the non-Rayleigh distributed scenarios emulated will depend in a complex way on the type of hardware change is made. Another drawback of these techniques is that a hardware modification of the cavity involves changes in the behaviour of the MSRC, which must be characterized for each proposed 
environment ( $Q$-factor, number of modes, lack of isotropicity...). In [23], a Rician-fading environment is obtained at the cost of varying the chamber characteristics and/or the antenna configuration, which implies an inherently higher hardware complexity to this option.

To overcome those limitations is the reason why two techniques are proposed in this chapter, which allow the emulation of different environments than Rayleigh fading by software processing of the samples. Such techniques have the advantage of allowing emulate fading environments with an accuracy determined by software, avoiding the inherent lack of control of hardware modifications. In the first part, it is proposed a technique that allows measurement of passive antennas under Rician environments by adding via software an in-phase component (LoS) to an actual measurement made in a Rayleigh environment (NLOS). The second proposed technique allows higher versatility, and can emulate arbitrary field distributions for both passive and active metrics, by selecting a subset of samples of all samples measured in a complete sequence of stirring.

\subsection{Arbitrary Rician Distributed Fading Emulation: The Offset Technique}

In this section, a novel processing technique which allows for the emulation of Rician-fading environments from the data taken in a MSRC with Rayleigh-fading environments. The method is not intended to describe in detail the response of a device to a change in the angle of arrival ( $A \circ A)$, but rather uses a deterministic component of a Rician signal via post processing to observe the associated changes in the conventional post-processing techniques used to determine general MIMO parameters of the device, such as diversity gain and MIMO capacity. The method allows the MIMO designer to evaluate several Rician $k$-factors environments with a single measurement set in the MSRC. With this technique a considerable reduction of chamber cost and complexity as well as measuring time is provided, alleviating the hardware complexity of other techniques.

\subsubsection{Emulating and measuring technique}

Measurements illustrated in this section have been performed with the EMITE Ing 8x8 MIMO Analyzer Series E200a in connection to the Rohde \& Schwarz ZVRE Vector Network Analyzer (9 kHz to $4 \mathrm{GHz}$ ). The MIMO Analyzer is a second generation twocavity MSRC with external dimensions of $0.82 \mathrm{~m} \times 1.275 \mathrm{~m} \times 1.95 \mathrm{~m}, 8$ exciting antennas, polarization stirring due to aperture-coupling and to the different orientation of the antenna exciting elements, 3 mechanical and mode-coupling stirrers, 1 holder-stirrer and variable iris-coupling. The MIMO Analyzer was set-up for 3 holder positions with 
15 different mechanical stirrer positions for each holder position, 12 iris-coupling aperture stirring and $20 \mathrm{MHz}$ frequency stirring. Measurements were performed at $1800 \mathrm{MHz}$ and half-wave dipoles were used as MIMO antennas. A sketch of the measurement setup can be observed from Fig. 35.

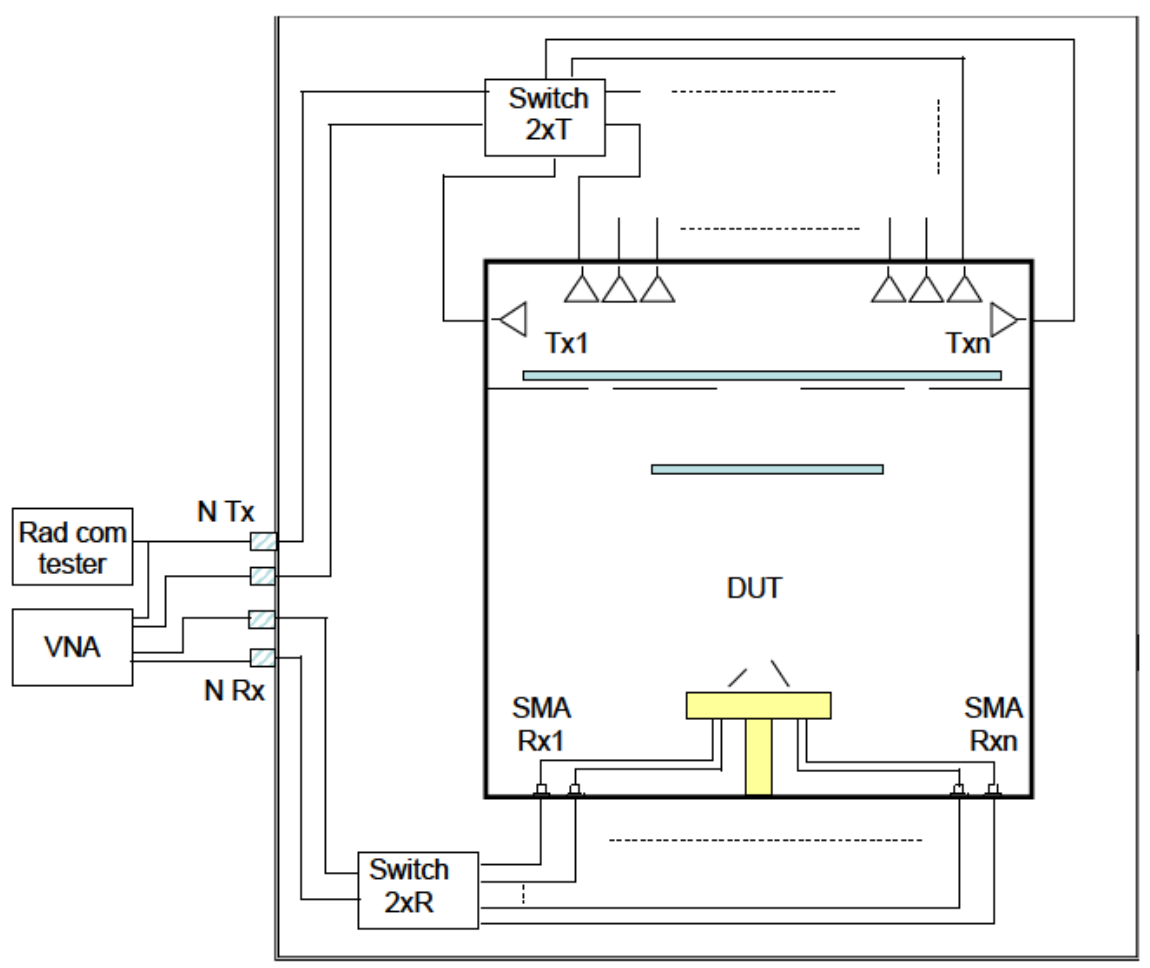

Figure 35. Sketch of measurements with the E200 MIMO Analyzer.

The $k$-factor, defined as the ratio of the direct path component to the scattered component can be calculated from measured S-parameter as it was seen in Chapter 3, according to the following expression [23],

$$
k=\frac{\text { direct component }}{\text { scattered component }}=\frac{d_{R}^{2}}{2 \sigma_{R}^{2}}=\frac{\left(\left|\overline{S_{21}}\right|\right)^{2}}{\overline{\left|S_{21}-\overline{S_{21}}\right|^{2}}}
$$

Where $S_{21}$ is the measured parameter in the MSRC for each antenna pair, $\sigma_{R}$ is the radius of cluster data and $d_{R}$ is the distance of the centroid of cluster from the origin. $\mathrm{A}$ scatter plot would result in the data clustered in a circle and centered about the origin for pure Rayleigh-fading environments $(k=0)$. As the direct line of sight (LoS) energy became comparable to the non-LoS energy, the cluster of data would move away from the origin, and the fading environment becomes a Rician one. Scatter plots are useful not only for identifying the LoS behaviour of the fading environments, but also to quantify the data dispersion through the $k$-factor. The data set measured with the chamber has to be large enough to ensure a Rayleigh-fading scenario. While this is a typical use of MSRCs, it also means that both the direct and non-direct coupling paths are included in the data set in a more complex mode-coupling multicavity MSRC. It is clear from eqn. (61) that by increasing the direct component the $k$-factor will increase. 
Consequently, we can move the cluster data away from the origin by adding an offset continuous component. The offset has to be a complex number by,

$$
\text { offset }=\mid \text { offset } \mid * \cos \left(\text { offset }_{\varnothing}\right)+i *|o f f \operatorname{set}| \sin \left(\text { offset }_{\varnothing}\right)
$$

For a target $k$-factor $\left(k_{\text {target }}\right)$, the required offset can be defined by,

$$
\mid \text { offset } \mid=\sqrt{k_{\text {target }} * 2 \sigma_{R}^{2}-\left|d_{R}\right|}
$$

The phase of the offset in Eqn. (63) is obtained from the averaged phase of all data samples in the initial set. Eqn. (63) can be re-written in terms of the S-parameters by,

$$
\mid \text { offset }\left|=\sqrt{k_{\text {target }} * 2\left\langle\left|S_{21}-\left\langle S_{21}\right\rangle\right|^{2}\right\rangle}-\right|\left\langle S_{21}\right\rangle \mid
$$

In order to obtain the desired results, the added offset would have to be phasecoherent to the selected radius in the way,

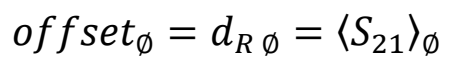

The calculated offset has to be added to all samples that are used for the Rician emulation. In this way the new Rician-fading samples keep the standard deviation of the original Rayleigh-fading ones, and therefore the distance can be altered to be adapted to the target $k$-factor. Fig. 36 depicts several scatter plots for the original Rayleigh-fading samples and different Rician-fading ones with $k=5,15$ and 100 using the proposed technique.

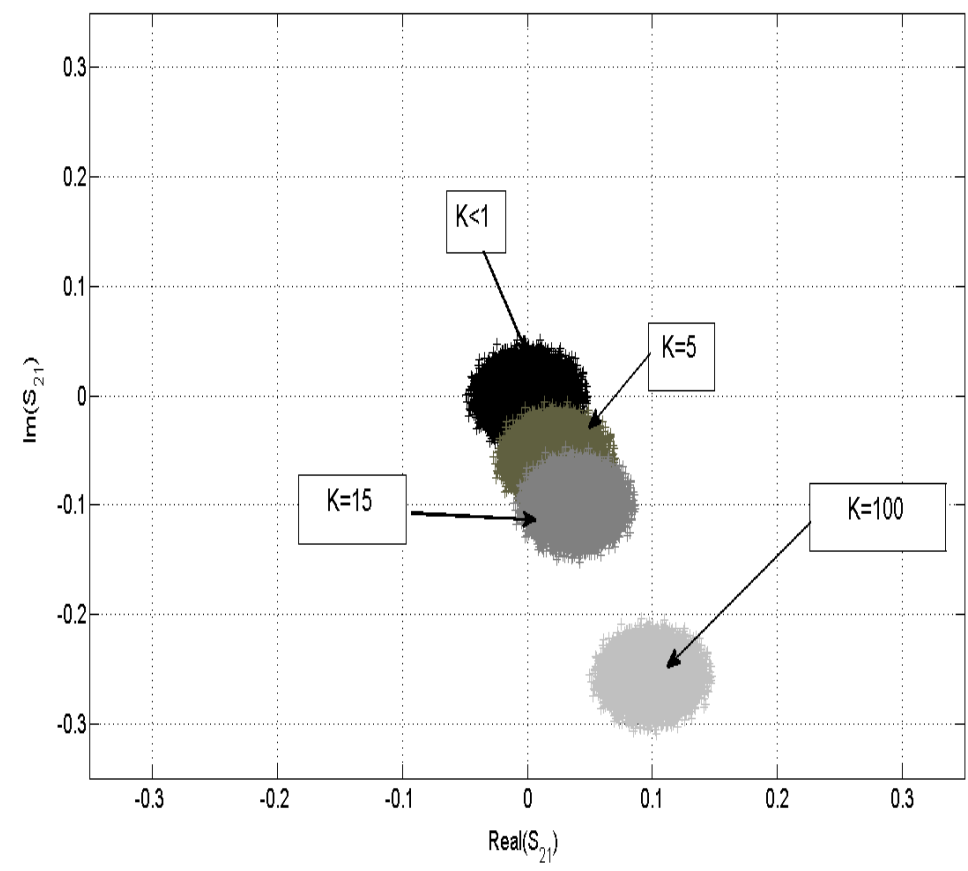

Figure 36. Scatter plots for mode-stirred and mode-processed data. 
It is clearly observed from this figure that the modified samples keep the standard deviation while their radius varies in a proportional way to the target $k$-factor. Fig. 37 shows a comparison between measured histograms for diverse sample sets with their associated probability distribution functions and several emulated sample sets. Originally-emulated samples can clearly be associated to a Rayleigh-fading scenario, while modified samples conform very well to measured Rician-fading scenarios with $k=5,15$ and 100. It seems clear, however, that the method relies on the previous estimation of the initial $k$ factor of the sample set. Consequently, the uncertainties of this method are linked to the uncertainties of the method employed to estimate the initial $k$ factor of the sample set.

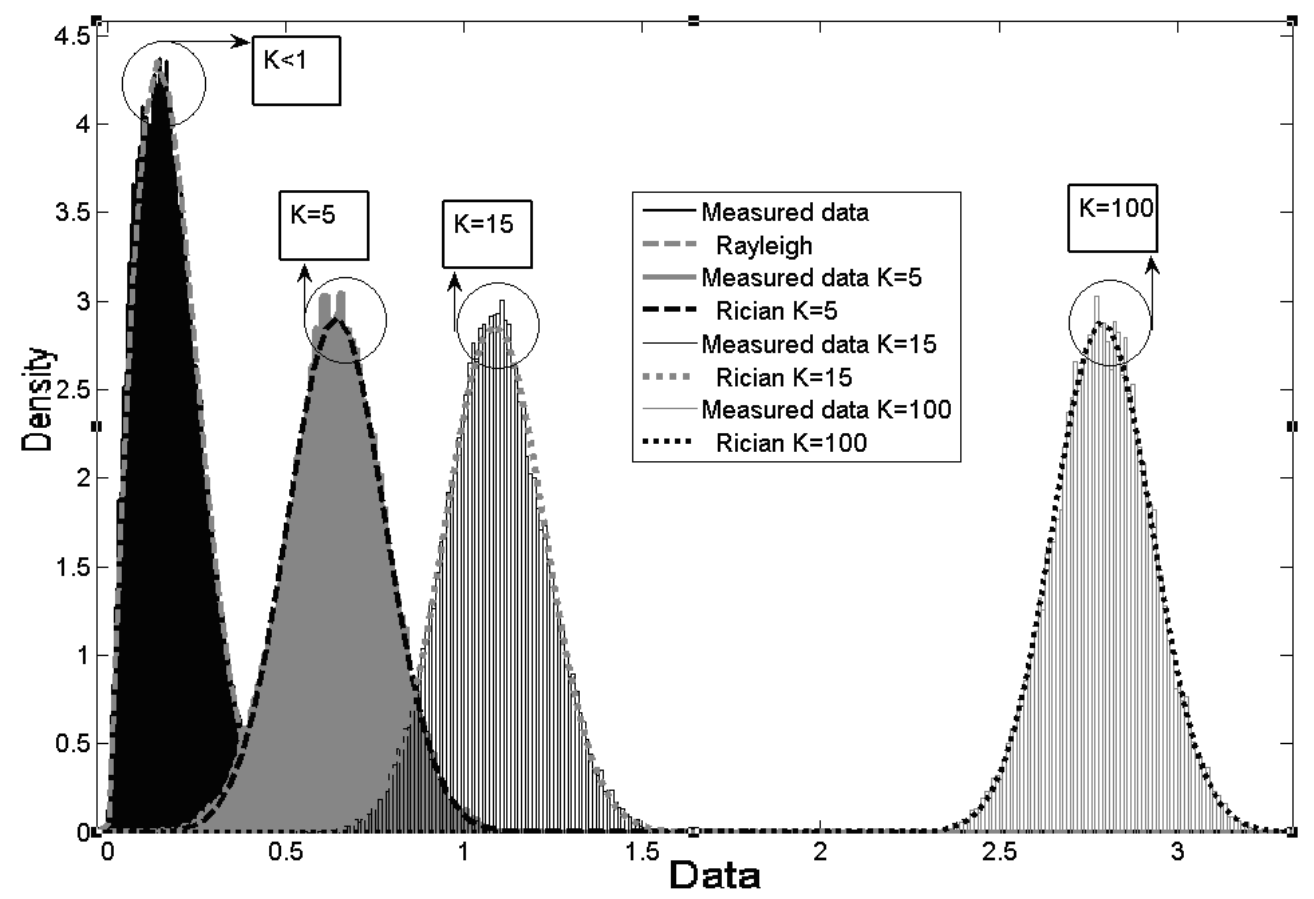

Figure 37. Histograms for measured and modified data.

In order to validate the proposed technique, 3 different initial $3 \times 3$ MIMO systems were measured. Three $\lambda / 2$ dipoles in a vertical position were employed as antennas in reception. The difference between the tested arrays was the spatial separation between the receiving dipoles, as shown in Table 20. The initial measured $k$-factor was always below 0.0001 for the 3 systems, that is clearly a Rayleigh-fading environment. The initial $k$-factors were then incremented slightly to between $10^{-3}$ and $10^{3}$ using the proposed technique. In this way, with only 3 system measurements in a Rayleighfading environment, it was possible to emulate and evaluate their performance for any Rician-fading environment. This drastically reduces both R\&D costs and time. The emulated results were compared with the outdoor measurements results in [108]. This comparison is shown in Fig. 38. Good agreement is observed between outdoor measurements and measurements emulated with the proposed technique. Thus, this method does not intend to describe the specific response of the device to a change in 
the specific AoA characteristic of a fading scenario, but rather to describe the changes in the general performance of the device when the scenario contains an increased LoS component. In Fig. 38, the method closely follows the real evaluation of a device for changing $k$ factors in outdoor measurements made in [108]. This is clear evidence that the technique represents a step forward to the goal of obtaining real-world performance from a MSRC test.

TABle 18. Measured MiMO arRays

\begin{tabular}{c|c|c}
\hline \hline System & $\begin{array}{c}\text { Spatial separation between adjacent } \\
\text { antennas }(\mathrm{d} / \mathrm{\lambda})\end{array}$ & $\begin{array}{c}\text { Initial measured K- } \\
\text { factor }\end{array}$ \\
\hline $\mathrm{A}$ & 0.01 & $<0.0001$ \\
$\mathrm{~B}$ & 0.05 & $<0.0001$ \\
$\mathrm{C}$ & 0.10 & $<0.0001$ \\
\hline \hline
\end{tabular}

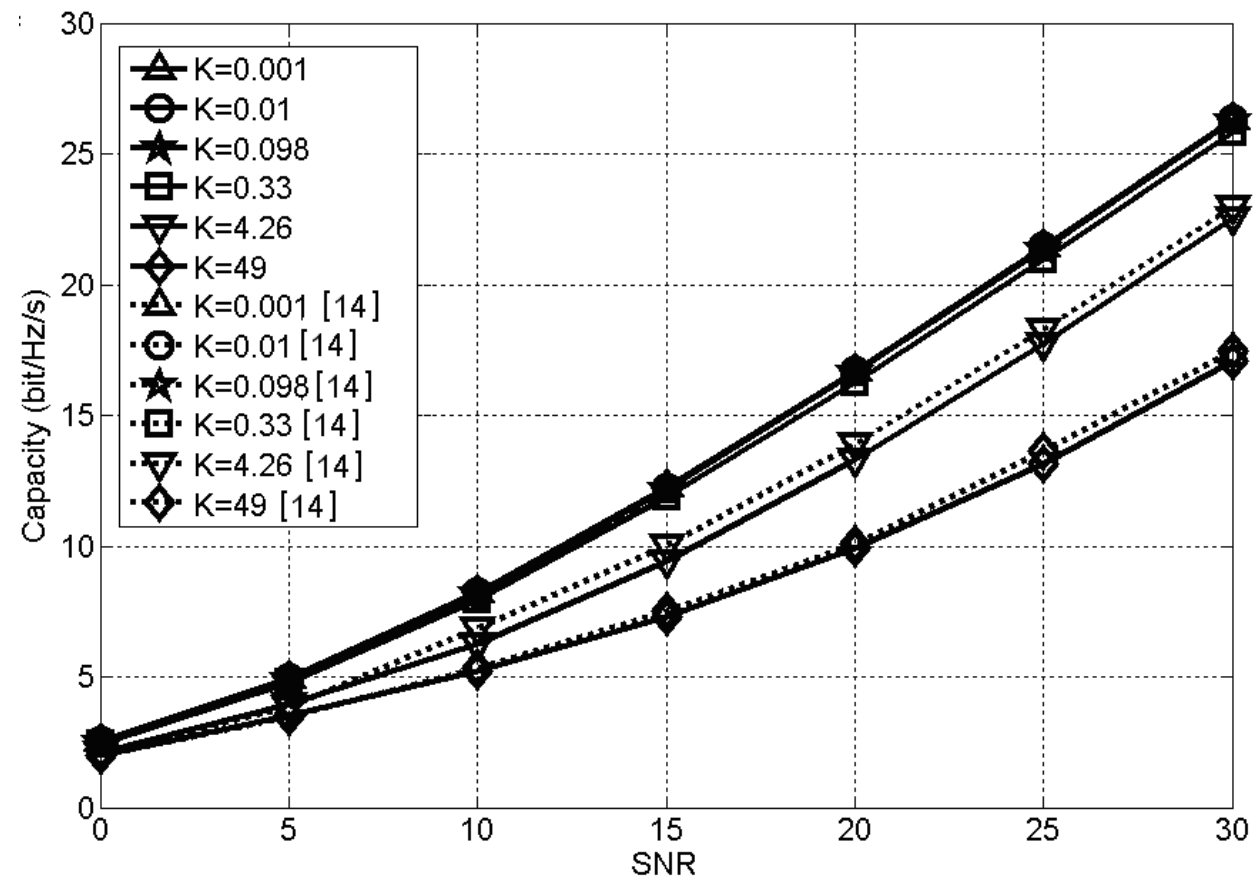

Figure 38. Measured and emulated capacity vs. SNR for 3x3 MIMO system A.

\subsubsection{Validation of the Technique}

\subsubsection{Correlation}

A traditional parameter to be analyzed is the correlation between antennas, which gives us an idea of signal similarities. With the aid of the proposed technique, the correlation properties in terms of the fading environment could be studied. In this way we can analyze how does the correlation coefficient change from Rayleigh-fading environments $(k=0)$ as $k$-factors are increased. Fig. 39 shows the correlation coefficient between adjacent antennas for the different systems in Table 18. From this figure one 
can observe that different correlation characteristics exist for the different systems. As it was expected, the system with highest correlation coefficient is system $A$. This is because of its smallest separation distance between adjacent vertically-polarized dipole antennas, which leads to the highest correlation coefficient. In consequence, system $\mathrm{C}$ depicts the lowest correlation coefficients. It is also important to note from figure 39 that the correlation coefficients change with changes in the $k$-factor. While the increment of correlation in system $A$ with increasing $k$-factor is almost imperceptible (0.97 to 0.98 ), system $C$ nearly doubles this parameter ( 0.18 to 0.39 ), while system B provides for a $22.4 \%$ increment ( 0.58 to 0.71 ).

Therefore, one can conclude that when increasing the $k$-factor the correlation coefficient also increases, and this increment is more noticeable when the correlation factor is low. In other words, the influence of the $k$-factor is more important for systems with low correlation between adjacent antennas. It is also important to stress that the increase in the change in the correlation coefficient that occurs when varying the $k$-factor has a limitation. For $k$-factors below 0.01 the correlation remains constant. For $k$-factors between 0.01 and 0.1 the correlation increases very quickly with increasing $k$-factor. This $k$-factor range accounts for $10 \%$ of the total correlation increment. For $k$-factors between 0.1 and 10, the correlation increment slope is more pronounced, achieving in this $k$-factor range a $90 \%$ of the total increase in correlation. Finally, for $k$-factors above 10 the correlation coefficient again remains constant. This means that the environment influences the correlation factor in a specific $k$-factor range. In other words, the $k$-factor plays a role on correlation coefficients for $0.1<k$ factor<10. In this $k$-factor range a small change in the fading environment has a direct impact on correlation. Once the NLOS fading environment degraded to LoS with $k$ factor $>10$, a further degradation of the NLoS environment has no effect on the correlation coefficient. 


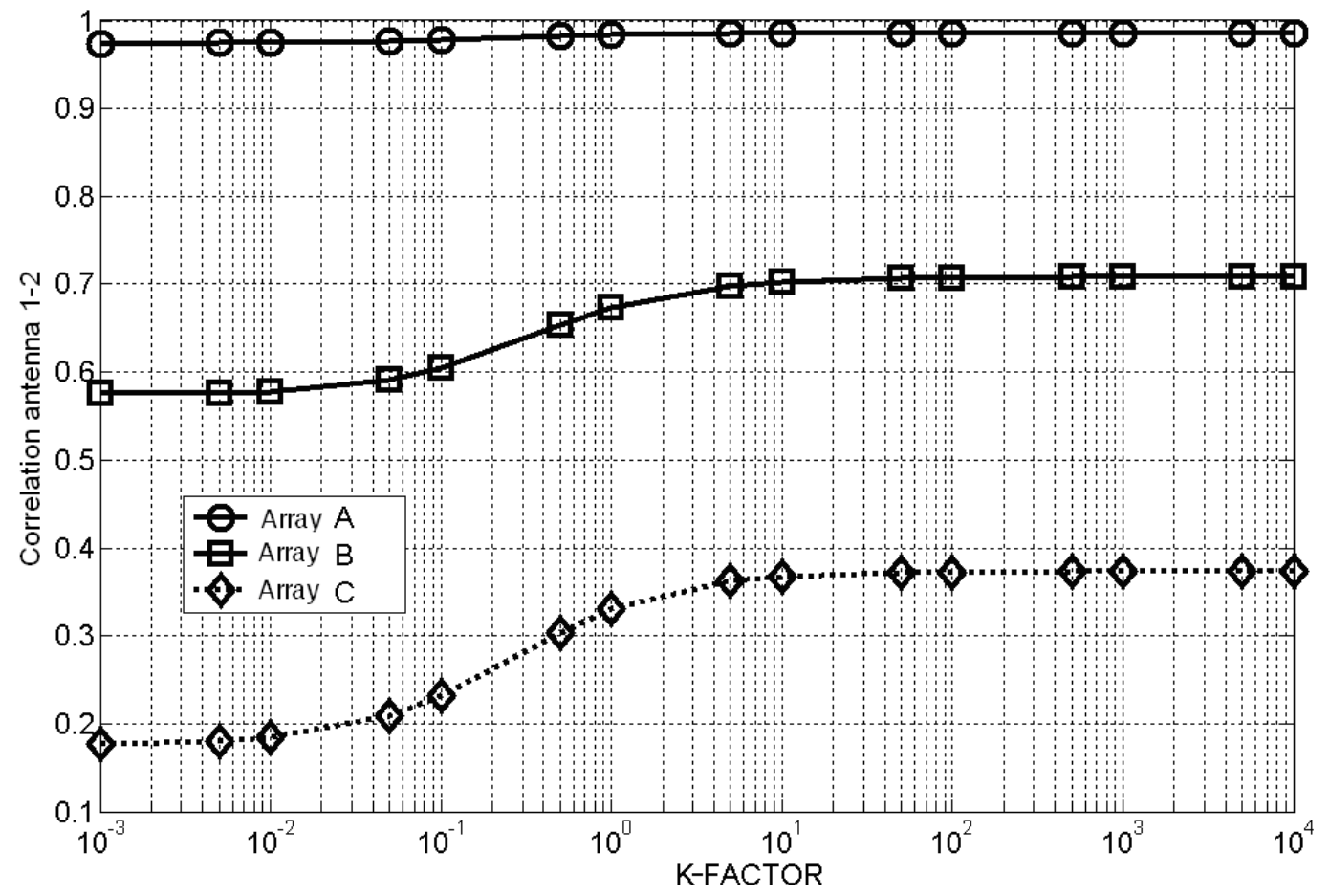

Figure 39. Emulated correlation coefficients between adjacent antennas for the MIMO systems in Table 20.

\subsubsection{2. $D G$}

With the proposed technique the influence of the $k$-factor on DG can also be analyzed. Fig. 40 shows the cumulative probability density function vs. relative power for system B with different $k$-factors. From this figure it becomes clear that the different curves with $k$-factors $<1$ cannot be differentiated, representing a typical Rayleigh-fading distribution. From $k$-factors between 1 and 10000 , the curves approach a perpendicular line to the $x$-axis. Consequently, diversity gain decreases with increasing $k$-factors, which was expected but tested in a MSRC for the first time. Fig. 41 depicts the DG versus $k$-factor with probability level as parameter for all tested systems. In a similar way to what happened for correlation, Fig. 41 also provides for a three-stage effect of the $k$-factor, this time for DG. Roughly constant DG values are observed for $k$-factors below 0.1 . From $k=0.1$ until $k=1$ a moderate decline in the DG happens. This decline becomes more pronounced for $k=1$ until $k=10$. Finally, for $k>10$ there is no diversity gain as this value approaches 0 for any given probability level and array type. Fig. 41 also offers an interesting comparison between tested systems. For a $5.8 \mathrm{~dB}$ of DG and a cumulative probability of $1 \%$, the highest possible $k$-factor is 0.001 , 2 and 3.5 for system $A, B$ and $C$ respectively. This means that system $C$, with the lowest correlation but the largest volume, represents a stronger design against a degradation of the NLOS characteristics of the fading scenario. Yet, it is also observed from these figures that the inherent advantages of system types are mitigated with increasing $k$ factor. 


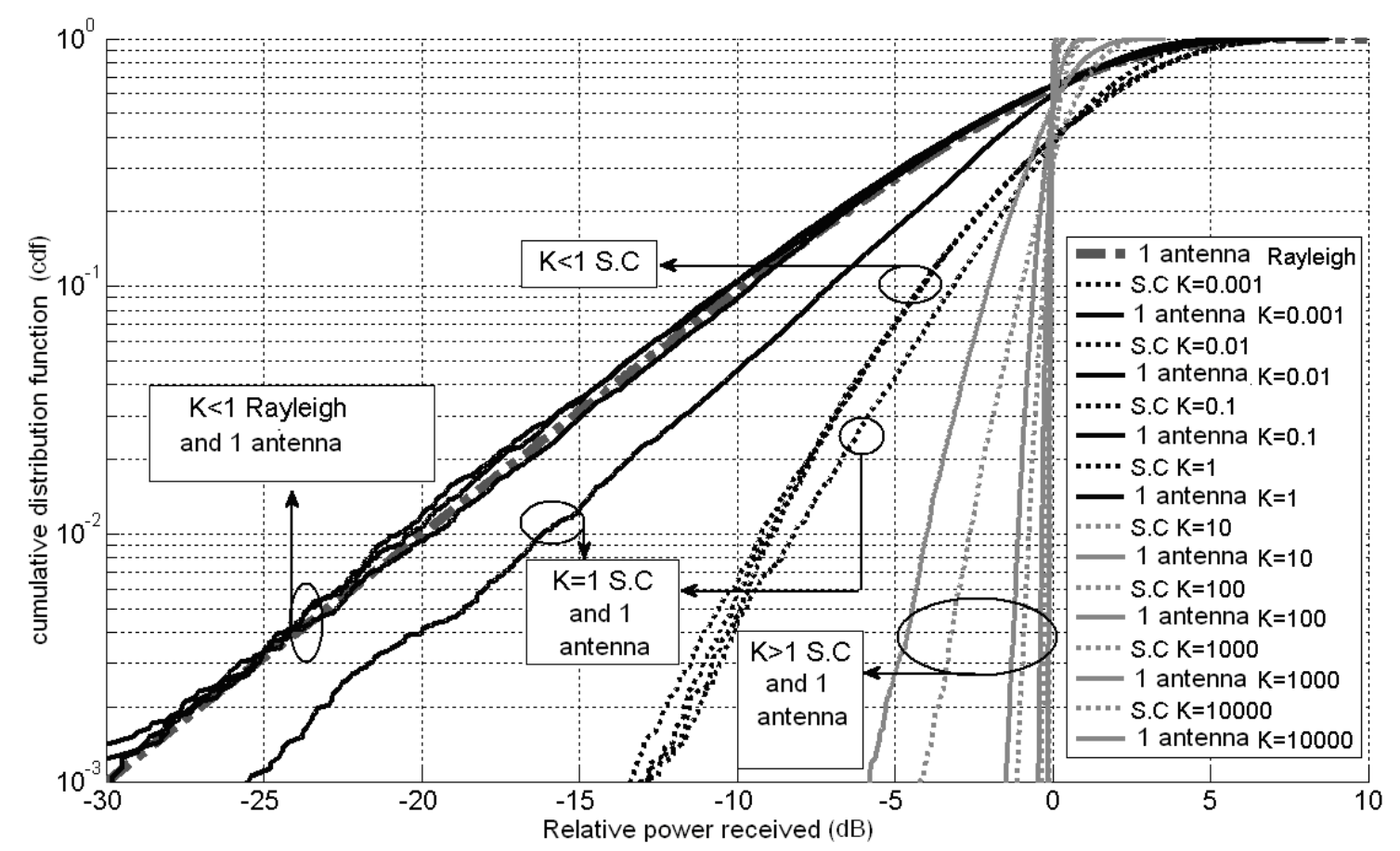

Figure 40. Cumulative probability density function vs. relative power for system B with different $k$ factors.

For example the difference between selection combined of system $A$ and $C$ for $k=0.1$ and a cumulative probability of $0.2 \%$ is $9 \mathrm{~dB}$, while the difference between these same systems for $k=10$ and the same probability is only $1 \mathrm{~dB}$. This means that with $k=0.1$ the influence of the array geometry is very important, while the influence of the array geometry for $k=10$ onwards is very small. This could be taken into account by MIMO engineers to decide when it may be necessary to use only a selection of separated antennas or the whole receiving array as a functions of fading scenarios where the system is under operation. As a general recommendation, it can be said that for $k$-factors below 10 , an increase in the spacing between antennas may be useful. Also as a general rule, it can be established that for lower cumulative probability and lower $k$-factors, the effect of MIMO array geometry on diversity gain is more pronounced. 


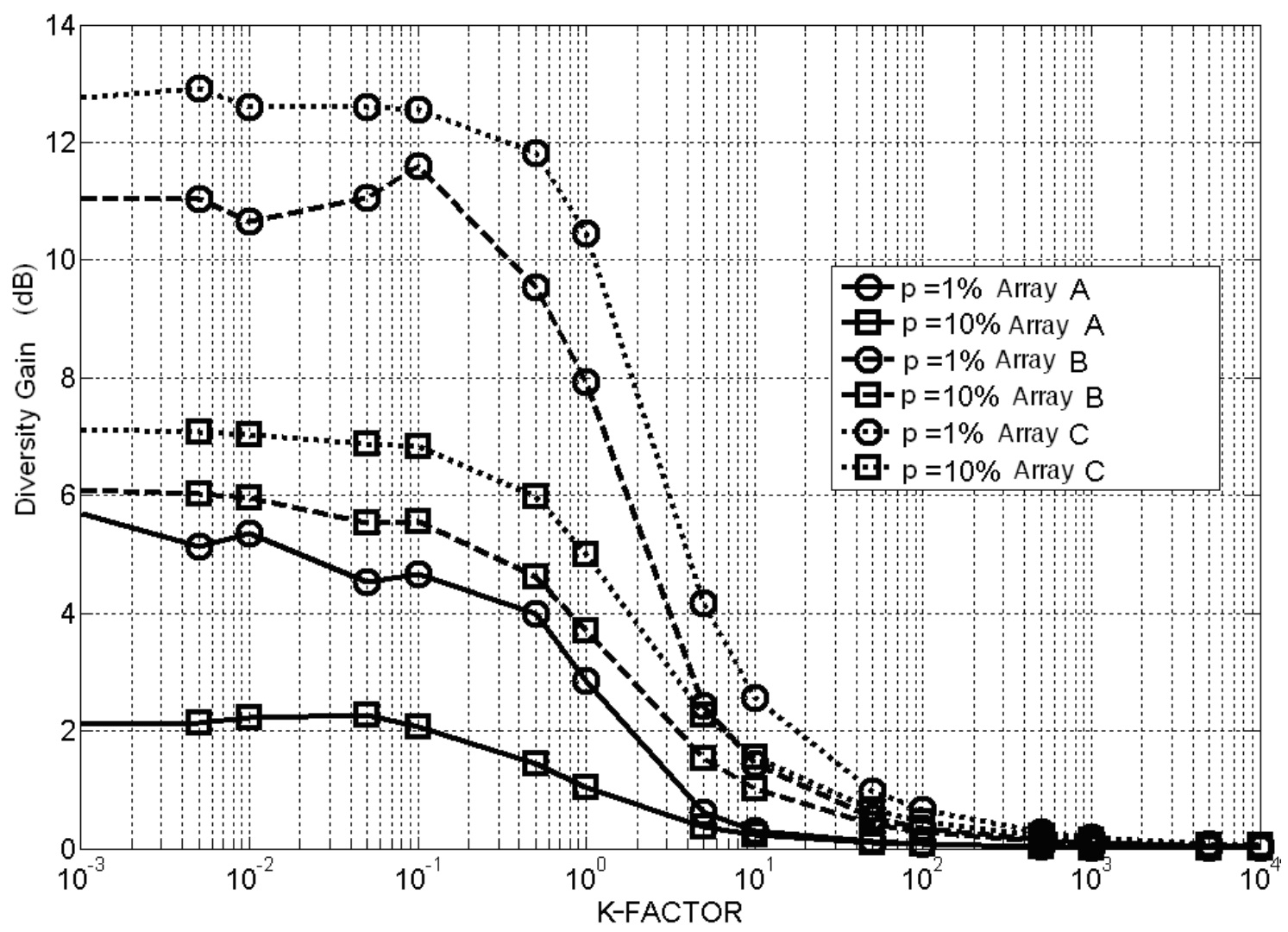

Figure 41. DG versus $\boldsymbol{k}$-factor with probability level as parameter, for all tested systems.

\subsubsection{MIMO Capacity}

Fig. 42 shows the measured capacity for all tested systems vs. SNR for different $k$ factors. Fig. 43 depicts the same measured capacity vs. $k$-factor for different SNR values. Fig. 42 clearly shows that system capacity decreases with increasing $k$-factor, as expected. The decrement is more noticeable for very high SNR values, as it was also expected. As an example, for system $B$ and a $S N R=30 d B$, the capacity loss with regard to the case with $\mathrm{K}=0.001,1$ and 10000 is $1.6 \mathrm{bit} / \mathrm{s} / \mathrm{Hz}(7 \%), 6.1 \mathrm{bit} / \mathrm{s} / \mathrm{Hz}(26.9 \%)$ and 11 $\mathrm{bit} / \mathrm{s} / \mathrm{Hz}$ (48.7\%), respectively. For very high $k$-factors all systems exhibit the same capacity, as it was also expected. Similarly, the minimum SNR required for achieving a specific system capacity can be extracted from Fig. 43. For example, in order to reach a system B capacity of $10 \mathrm{bits} / \mathrm{s} / \mathrm{Hz}$, a SNR=15, 16.3, 20, 23.2 and 25 would be required for $k$-factors of $0.001,0.1,1,10,100$ and 1000, respectively. Some examples of SNR values required to reach specific capacities at different $k$-factors are listed in Table 19. 


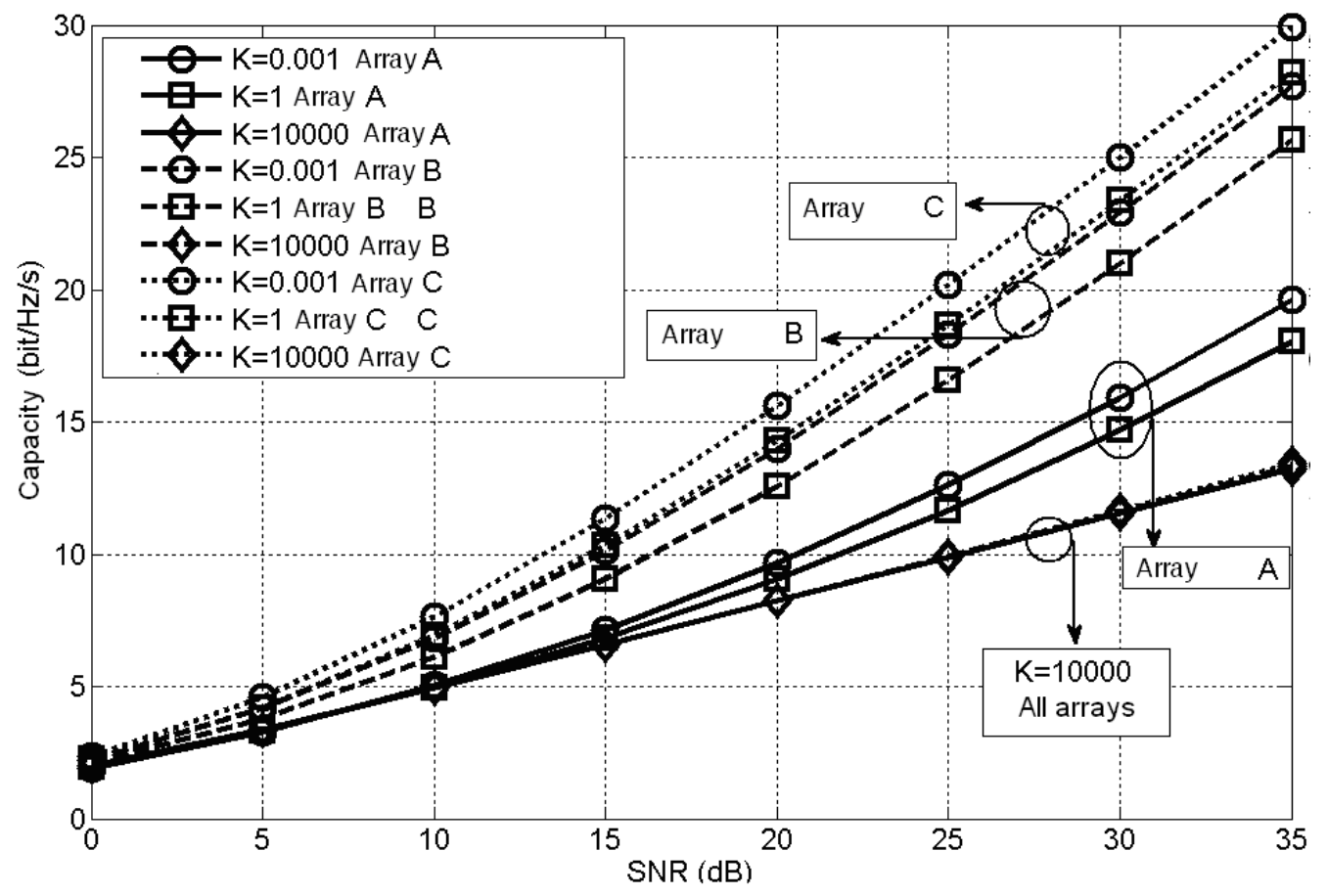

Figure 42. Measured capacity of all tested systems vs. SNR for different $k$-factors.

For example, if a capacity greater than $15 \mathrm{bit} / \mathrm{s} / \mathrm{Hz}$ is required, this can be done with a $\mathrm{SNR}=25 \mathrm{~dB}$ and $k<3$ or a $\mathrm{SNR}=35 \mathrm{~dB}$ and $k<350$. This requirement would not be fulfilled for SNR<15 dB. Finally, it is also clear from Fig. 43 that for high $k$-factors and given a specific SNR value, all systems achieve the same capacity. The capacity increase when changing a MIMO system to a different one out of Table 18 is illustrated in Table 20 with respect to the $k$-factor. From this Table it is also extracted that the change from system $A$ to $B$ produce a considerable capacity increase (over 15\%) for $k$ factor $\leq 50$ with $S N R=35 \mathrm{~dB}$. The same change but with $S N R=15 \mathrm{~dB}$, however, provides for similar capacity increments but this time for $k$-factor $<0.5$. This means that larger spatial separations in MIMO arrays provide a better benefit for high SNR scenarios.

TABLE 19. REQUIRED SNR FOR DIFFERENT CAPACITIES.

\begin{tabular}{|c|c|c|c|c|c|c|c|c|c|}
\hline$k$-factor & \multicolumn{3}{|c|}{$k=0.001$} & \multicolumn{3}{|c|}{$k=1$} & \multicolumn{3}{|c|}{$k=10^{4}$} \\
\hline System & $A$ & $B$ & $C$ & $A$ & $B$ & $C$ & $A$ & B & C \\
\hline $\begin{array}{l}\text { Cap. } \\
\text { bits/s/Hz }\end{array}$ & $\begin{array}{l}\text { SNR } \\
(d B)\end{array}$ & $\begin{array}{l}\text { SNR } \\
(\mathrm{dB})\end{array}$ & $\begin{array}{l}\text { SNR } \\
\text { (dB) }\end{array}$ & $\begin{array}{l}\text { SNR } \\
(\mathrm{dB})\end{array}$ & $\begin{array}{l}\text { SNR } \\
(d B)\end{array}$ & $\begin{array}{l}\text { SNR } \\
(\mathrm{dB})\end{array}$ & $\begin{array}{l}\text { SNR } \\
(\mathrm{dB})\end{array}$ & $\begin{array}{l}\text { SNR } \\
(\mathrm{dB})\end{array}$ & $\begin{array}{l}\text { SNR } \\
(\mathrm{dB})\end{array}$ \\
\hline 10 & 20.3 & 15 & 13.4 & 22.2 & 16.1 & 14.7 & 25 & 25 & 25 \\
\hline 15 & 28.1 & 21.3 & 19.2 & 30.4 & 23.1 & 20.9 & ---- & ---- & ---- \\
\hline 20 & ----- & 27.4 & 24.9 & ----- & 28.6 & 26.9 & ---- & ---- & ---- \\
\hline
\end{tabular}




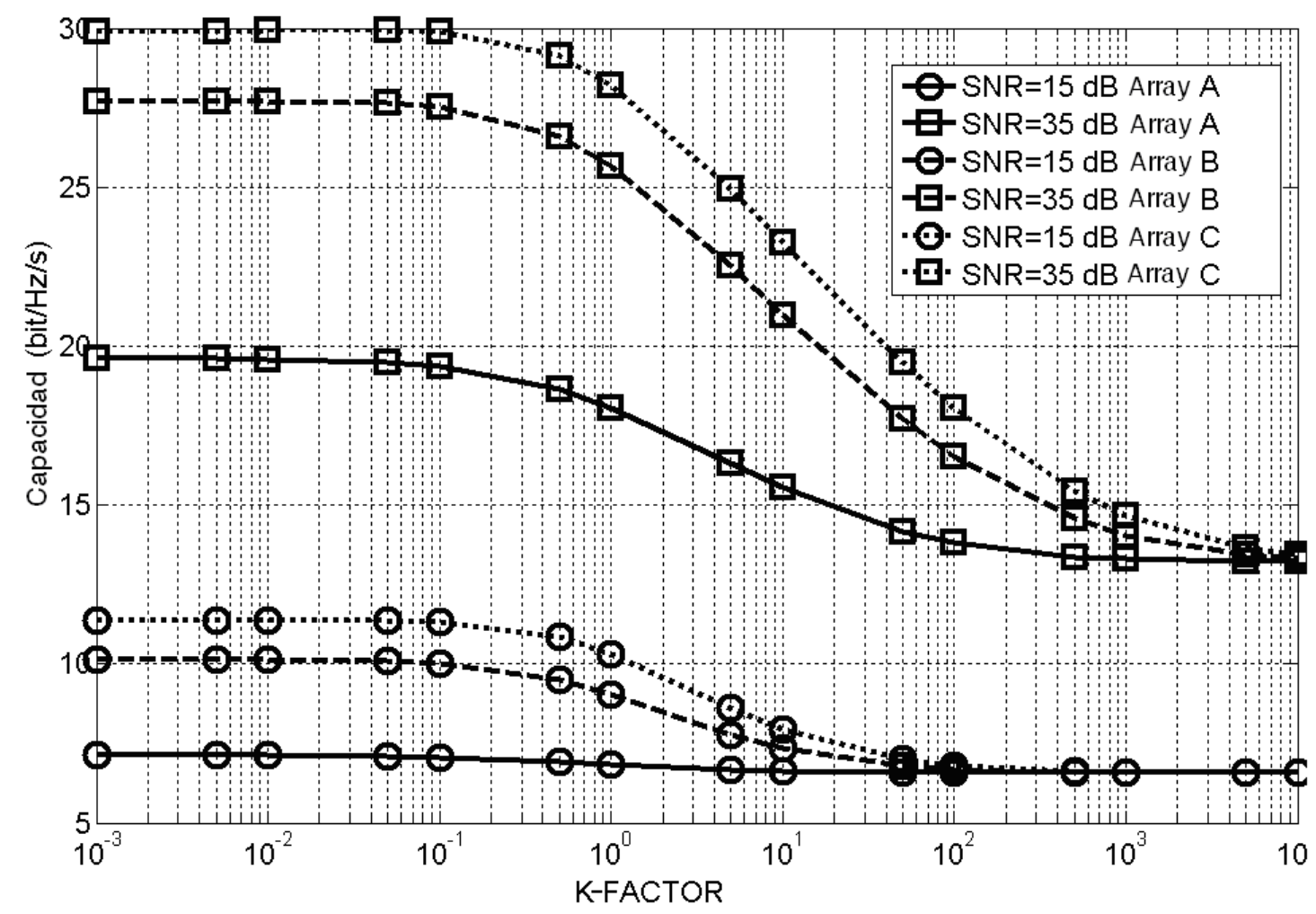

Figure 43. Measured capacity of all tested systems vs. $\boldsymbol{k}$-factor for different SNR values.

TABle 20. CAPACITY INCREASE (\%) WHEn CHANGING MIMO SYSTEMS

\begin{tabular}{|c|c|c|c|c|}
\hline SNR & \multicolumn{2}{|c|}{$15 \mathrm{~dB}$} & \multicolumn{2}{|c|}{$35 \mathrm{~dB}$} \\
\hline$k$-factor & $A$ to $B$ & B to $C$ & $A$ to $B$ & B to $C$ \\
\hline 0.5 & 35.7 & 13.6 & 43.0 & 10.1 \\
\hline 5 & 14.5 & 7.5 & 38.8 & 11.0 \\
\hline 50 & 2.8 & 2.8 & 25.0 & 11.4 \\
\hline 500 & 0 & 0 & 6.5 & 5.4 \\
\hline 5000 & 0 & 0 & 0.7 & 0.7 \\
\hline
\end{tabular}

\subsection{Arbitrary Fading Emulation: The Sample Selection Technique}

Unlike traditional MSRC, where only one cavity is used, second generation MSRCs may contain more than one metal cavity. Cavities are coupled by diverse means, and a rich fading environment consisting of diverse clusters with different fading characteristics can be obtained. The MSRC also contains a set of mode stirrers that change the boundary conditions of the main cavity within the chamber. This causes a multireflective environment that is repeatable and can be statistically studied. MSRCS can be used as a very fast, easy, and accurate tool to measure a wide variety of MIMO parameters. There is yet a shortage of studies of their delay spread and isotropicity properties. In consequence, its potential ability to emulate realistic environments is normally put into jeopardy and typically degraded to that of simple single-cavity 
MSRCs. Single cavity MSRCs typically provide a fading environment that is isotropic, and the amplitude of the signal is Rayleigh-distributed.

Diverse isolated enhancements can be found in the literature for MSRCs, as those studied in Chapter 3. However, changes in the antenna configuration and/or in the chamber itself were required. The use of multiple cavities on MSRCs can provide for a more complicated fading emulation. With multiple cavities, MSRCs can reduce the typically high-elevation angular spread of MSRCs, which approaches the emulated scenario to that typically encountered in a city street microcell when the mobile station gets farther away from the base station [8]. Thus, multiple cavities MSRCs abandon the typical Clarke's model followed by single-cavity MSRCs. In this section a novel method to emulate more realistic arbitrary-fading environments using a twocavity MSRC is presented. This is done by using a sample-selection technique. By keeping or discarding specific samples, it is possible to achieve a new subset with different fading distribution characteristics. The new subset consists only of unmodified measured samples forming a statistical ensemble different from Rayleigh. No hardware changes are required, neither in the chamber itself nor in the antenna configuration. The technique demonstrates the versatility of MSRCs to emulate realistic MIMO scenarios.

\subsubsection{Emulating and Measuring Technique}

The sample-selection technique consists of extracting the subset that conforms to a specific target fading statistical ensemble from the whole sample set measured in the MSRC. An algorithm has to decide whether each measured sample has to be kept or discarded. In this section, an evolutionary method of a genetic algorithm (GA) [109] is employed to optimize the fitness between the density function of an ensemble of samples and a Rician-fading target density function that was a priori defined. GAs operate on a population of potential solutions applying the principle of survival of the fittest to produce successively better approximations to a solution. At each generation of a GA, a new set of approximations is created by the process of selecting individuals according to their level of fitness in the problem domain and reproducing them using operators borrowed from natural genetics.

This process leads to the evolution of populations of individuals that are better suited to their environment than the individuals from which they were created, just as in natural adaptation. Inspired by the process of biological evolution, GA evolutionary methods employ stochastic search for obtaining the optimal solution. The key property of evolutionary methods is that occasionally very large changes in the solution are introduced. The presence of such large changes and random variations implies that evolutionary methods can find good solutions even in extremely complex 
discontinuous spaces or fitness landscapes that are hard to address by techniques such as gradient descent.

This is why the GA-evolutionary algorithm was employed in this section. The GA implemented in the Matlab toolbox gads was initially used. The Matlab-based GA allows for a combination of the innate advantages of GA optimization with all the functions implemented in all the other toolboxes of this software. The application of GAs to the sample-selection problem consists of assigning one binary variable ( 0 or 1 ) to each sample, as it happens in the typical GA binary string called "chromosome," representing whether this sample will be in the final solution or not. The GA allows for a bit string to embrace the final solution, which can be quickly obtained from the chromosome population of the genetic algorithm. With an initial Rayleigh-fading distribution from the complete sample set typically emulated by an MSRC, the error in the achieved target Rician distribution is analyzed by calculating the mean-square error between the achieved and the target distributions as

$$
\varepsilon_{d}=\frac{1}{Q} \sum_{x=0}^{Q}\left(f(x)-f_{\text {target }}(x)\right)^{2} d x
$$

Where $Q$ is the number of elements in the array, $d_{x}$ is the distance between two elements in the PDF, $f(x)$ is the target fading distribution, $f_{\text {target }}(x)$ and is the achieved target distribution with a subset of measured samples. In addition to $\varepsilon_{d}$, the relative amount of discarded data is noted since it will affect the final accuracy.

The employed error (fitness) function within the GA was a sum squared error (SSE)based function, which can be easily implemented at a reasonable computation complexity for the task at hand. Since, for the sample-selection problem, we are looking for best fits to target density functions, which range from 0 to 1 , the conventional SSE formula was modified as

$$
\operatorname{error}_{i}=\frac{\sum_{i=1}^{n}\left(f_{i}(n)-f_{\text {target }}(n)\right)^{2}}{\sum_{i=1}^{n}\left(f_{\text {target }}(n)\right)^{2}}
$$

where $n$ is the number of bins used to calculate the density function.

To achieve a reasonable ratio between computing time and accuracy, the fitness value was limited to 0.01 by default. Since the phase distribution in an MSRC is typically uniform over $2 \pi$, in this study the goal includes maintenance of this phase distribution on the final subset. In a more general case also, the phase of the data can be included in the PDF. 


\subsubsection{Sample Selection Algorithms}

The sample selection process can be implemented in different ways. Depending on the algorithm implemented, both the convergence time required to obtain a given accuracy, and the number of samples selected in the final ensemble may vary. This section proposes three different approaches to solving this problem, and discusses the accuracy and the percentage of discarded samples for each of the proposed algorithms.

\subsubsection{The Single Step Algorithm}

The first algorithm in our study is inspired by a probability density point of view [110]. In this algorithm we define a relationship between the initial distribution and its target distribution counterpart by

$$
f_{\text {initial }}\left(S_{21}\right) \cdot D\left(S_{21}\right)=f_{\text {target }}\left(S_{21}\right)
$$

where $f_{\text {initial }}\left(S_{21}\right)$ is the initial PDF, $D\left(S_{21}\right)$ is a weighting function and $f_{\text {target }}\left(S_{21}\right)$ is the target PDF. The weighting function $D$,

$$
D\left(S_{21}\right)=\frac{D_{1}\left(S_{21}\right)}{\max \left(D_{1}\left(S_{21}\right)\right)}
$$

where the amplitude of function $D$ is normalized to have a value between zero and unity.

$$
D_{1}\left(S_{21}\right)=\frac{f_{\text {target }}\left(S_{21}\right)}{f_{\text {initial }}\left(S_{21}\right)}
$$

In the algorithm, the $i$ :th measured sample with an amplitude of $S_{21}{ }^{i}$, will be kept if $D\left(S_{21}{ }^{i}\right)>U(0,1)$ is true (otherwise discarded). The notation $U(0,1)$ defines a random variable with uniform distribution between 0 and 1 . Therefore, the function $D\left(S_{21}\right)$ in the algorithm is used as a decision threshold for each sample such that it provides the probability of keeping the sample.

\subsubsection{The Iterative Genetic Algorithm}

In an attempt to get more accurate results, an evolutionary method of a genetic algorithm (GA) [109] is also employed to optimize the fitness between the density function of an ensemble of samples and a target density function. The GA implemented in the MATLAB toolbox named "gads" is initially used. This allows for a combination of the innate advantages of GA optimization with all the functions implemented in all the other toolboxes of MATLAB.

The application of GA to the sample selection problem consists of assigning one binary variable ( 0 or 1 ) to each sample, denoting whether the sample will be in the 
final set or not. The algorithm can be further constrained with a pre-defined minimum number of samples that we want in the final solution ensemble so as to conform to a set of goodness-of-fit tests. The employed error (fitness) function is a sum squared error (SSE) based function. Since for the sample selection problem we are looking for best fits to target density functions, which range from 0 to 1 , the conventional SSE formula was modified as,

$$
\varepsilon_{S S E}=\frac{\sum_{i=1}^{n}\left[f_{\text {final }}(i)-f_{\text {target }}(i)\right]^{2}}{\sum_{i=1}^{n}\left[f_{\text {target }}(i)\right]^{2}}
$$

Where $n$ is the number of elements wherein the density function is evaluated. The fitness value is set by the user as a calculation limit for the GA and Hybrid algorithms.

\subsubsection{The Hybrid Algorithm}

The third algorithm employed in this study is a hybrid of the single step algorithm and the GA. First the single step algorithm is used to quickly determine the wanted set of data. The resulting subset of data with moderate accuracy is then used to feed the iterative GA in the second step. The second step is used to accurately determine the final data set. The GA hence has a smaller initial data set and a more accurate initial distribution of data, which leads to shorter computational times.

\subsubsection{4. $\quad$ Error Variable and Accuracy of Sample Selected Distributions}

To quantify the difference between the error of the target and sample selected distributions a mean sum-square error is defined as

$$
\varepsilon_{d}=\frac{1}{n} \sum_{i=1}^{n}\left(f_{\text {final }}(i)-f_{\text {target }}(i)\right)^{2} \Delta S_{21},
$$

where $n$ is the number of elements in the data vector and $\Delta S_{21}$ is the difference between two amplitude bins in the PDF, $f_{\text {final }}(i)$ is the final sample selected PDF. In addition to $\varepsilon_{d}$, the relative amount of remaining samples is noted, since it will affect the accuracy.

A dominating type of error is the statistical inaccuracy of the measurement, which is dependent of the number of independent samples, $N_{\text {ind }}$, in the initial distribution. When discarding some of the measurement samples in a sequence, the measurement accuracy is affected, but not necessarily decreased. If the discarded samples do not contribute to the desired target distribution, the accuracy can actually be improved. In order to see how the accuracy is affected by the sample selection technique, we choose to study the accuracy of the average power as a figure of merit for final target 
distribution accuracy. MSRCs are often used to measure the antenna radiation efficiency, which is proportional to the average power of the measured sequence.

In this work accuracy of average power is defined as ratio between the standard deviation of the average power and the mean power. How the sample selection technique affects the accuracy is found by deriving and comparing this ratio before and after applying the sample selection technique.

It is assumed that the measured sequence before applying the sample selection is Rayleigh-distributed. For an exponentially distributed random variable, the standard deviation $s$ and the mean value $\mu$ are equal. Furthermore, the central limit theorem states that the standard deviation is proportional to the inverse of the square root of the number of independent samples $N_{\text {ind }}$ [111], whereas the mean remains the same. We thus find that the relative accuracy for the initial data is

$$
\varepsilon_{\text {initial }}=\frac{s_{\text {initial }}}{\mu_{\text {initial }}} \cdot \frac{1}{\sqrt{N_{\text {ind,initial }}}}=\frac{1}{\sqrt{N_{\text {ind,initial }}}}
$$

where Nind,initial is the number of independent samples in the initial distribution.

Let's assume a Rician target distribution (ftarget). After having applied the sample selection technique, the amplitude becomes Rice-distributed, with parameters afinal and sfinal. The mean and the standard deviation of the power of a single sample within a Rice-distributed set are given by

$$
\begin{aligned}
& \mu_{\text {final }}=a_{\text {final }}^{2}+2 s_{\text {final }}^{2} \\
& s_{\text {final }, 2}=2 s_{\text {final }}^{2} \sqrt{1+\frac{a_{\text {final }}^{2}}{s_{\text {final }}^{2}}}
\end{aligned}
$$

This results in a relative accuracy of the final data

$$
\varepsilon_{\text {final }}=\frac{s_{\text {final }, 2}}{\mu_{\text {final }}} \cdot \frac{1}{\sqrt{N_{\text {ind }, \text { final }}}}=\frac{\sqrt{1+a_{\text {final }}^{2} / s_{\text {final }}^{2}}}{1+a_{\text {final }}^{2} /\left(2 s_{\text {final }}^{2}\right)} \cdot \frac{1}{\sqrt{N_{\text {ind,final }}}},
$$

where $N_{\text {ind,final }}$ is the number of independent samples in the final distribution. From (76), we can see that after applying the sample selection technique, an increased accuracy $\left(\varepsilon_{\text {initial }} \geq \varepsilon_{\text {final }}\right)$ can be achieved in MSRCs. Determining the number of independent samples in the final subset $\left(N_{\text {ind,final }}\right)$ is a key issue. The number of independent samples of the original sequence $\left(N_{\text {ind,initial }}\right)$ is estimated for instance by the techniques in [112] [113]. The oversampling ratio $X$ is defined as

$$
X=\frac{M_{\text {initial }}}{N_{\text {ind,initial }}},
$$


where $M_{\text {initial }}$ is number of measured samples in the initial set of data. When the algorithms are performing the sample selection, independent samples are discarded every time a consecutive sequence of measured samples which is longer than or equal to the oversampling ratio is discarded. For example, in the case of $X=3, N_{\text {ind, initial }}$ will not be reduced if 1-2 samples in a row are discarded. If 3-5 samples are discarded in row it reduces $N_{\text {ind,initial }}$ by one, and if 6-8 samples in row are discarded it reduces $N_{\text {ind,initial }}$ by two, and so on. This means that in some cases $N_{\text {ind,final }}$ can be equal to $N_{\text {ind,initial. }}$ In the worst case, on the other hand, $N_{\text {ind,initial }}$ is reduced by the same ratio as $M_{\text {initial }}$ (number of samples). That is to say,

$$
1 \geq \frac{N_{\text {ind,final }}}{N_{\text {ind,initial }}} \geq \frac{M_{\text {final }}}{M_{\text {initial }}}
$$

where $M_{\text {final }}$ is the number of samples after the sample selection algorithm.

When going from a Rayleigh distribution to a Rice distribution, the first factor $(s / \mu)$ on the right hand side of (75) will be lower in the final distribution than in the initial distribution, so it helps to reduce the error. The second factor in (75), on the other hand, typically increases the error because $N_{\text {ind,final }}<N_{\text {ind,initial }}$.

\subsubsection{Limit for the Proportion of Remaining Samples}

The sample selection technique is based on a simple concept, whereby only some of the samples measured are used to calculate the required antenna FoMs, so that the final set selected has certain characteristics (it follows a particular amplitude FDP). Thus, it is clear that the final number of samples after this process can only be maintained or decreased, depending on the PDF that is set as target, but could this reduction of samples to be very important. Therefore, the number of samples remaining after the sample selection process is a crucial parameter, in order to have measurements with good accuracy. This section will analyze the maximum limit to the number of samples remaining after the sample selection process. In order to make the study extendable to initial sample sets of any length, the proportion of remaining samples (denoted as $q$ ) is studied.

Are $f_{\text {initial }}(x)$ and $f_{\text {target }}(x)$ the PDF of the initial and target data (after sample selection), respectively. These functions have the usual properties of PDFs, that is, they are always positive and the integral over all arguments is unity.

The total number of samples before and after sample selection is denoted as $N_{\text {initial }}$ and $N_{\text {target }}$, respectively. The number of samples within a small interval $\Delta x$ at any $x$ value is $N_{\text {initial }} \cdot f_{\text {initial }}(x) \Delta x$ and $N_{\text {target }} f_{\text {target }}(x) \Delta x$, respectively. Naturally, the number of samples at any $x$ value can only remain the same or decrease as a result of the sample selection process. Consequently we have 


$$
N_{\text {target }} f_{\text {target }}(x) \leq N_{\text {initial }} f_{\text {initial }}(x)
$$

where $\Delta x$ is eliminated on both sides. As $f_{\text {target }}(x)$ is assumed to be different from $f_{\text {initial }}(x)$ (otherwise there is not sample selection), there will be $x$ values at which $f_{\text {target }}(x)>f_{\text {initial }}(x)$.

To fulfil Eq. (79), $N_{\text {target }}$ then has to be smaller than $N_{\text {initial }}$. It is the maximum ratio of $f_{\text {target }}(x) / f_{\text {initial }}(x)$ for any $x$ that dictates the necessary ratio of $N_{\text {target }} / N_{\text {initial }}$ for the condition in Eq. (79) to be satisfied. Defining the proportion of remaining samples $q$ as

$$
q=\frac{N_{\text {target }}}{N_{\text {initial }}}
$$

And substituting this in Eq. 79 gives the condition

$$
q \leq \frac{f_{\text {initial }}(x)}{f_{\text {target }}(x)}
$$

In a specific case, there can be some freedom in choosing $f_{\text {target }}(x)$, because PDFs often have one or several parameters and the requirements on $f_{\text {target }}(x)$ might not decide all of them. Remaining parameters should then preferably be set so that $q$ is optimized. The maximum value of $q$ is thus

$$
q_{\text {max }}=\min \left(\frac{f_{\text {initial }}(x)}{f_{\text {target }}(x)}\right)
$$

for all $x$, and for all $f_{\text {target }}(x)$ under the given constraints.

As it has been showed before, different algorithms can be used to perform de selection of the samples. However no assumptions about the employed algorithm have been made to obtain $q_{\max }$, so Eq. (82) is valid for any of the proposed algorithms.

\subsubsection{Sample Selection Performance}

\subsubsection{Measurement Setup}

Measurements illustrated in this section have been performed with the E200 8x8 MIMO Analyzer by EMITE Ing., in connection to the Rohde \& Schwarz ZVRE Vector Network Analyzer (9 kHz to $4 \mathrm{GHz}$ ). The E200 MIMO Analyzer is a MSRC with dimensions of $0.821 .2751 .95 \mathrm{~m}$, eight exciting antennas, polarization stirring due to aperture coupling and to the different orientation of the antenna exciting elements, three mechanical and mode-coupling stirrers, one holder-stirrer, and variable iriscoupling. As depicted in Fig. 44, the E200 MIMO Analyzer is composed of two cavities that are coupled through a slotted metallic plate.

Accurate control over the slots apertures allow for a complex coupling structure from the upper cavity, where the transmission antennas are placed, to the lower main cavity, wherein the MIMO device under test (DUT) is placed. The E200 MIMO Analyzer was set up for three holder positions with 15 different mechanical stirrer positions for 
each holder position, 12 iris-coupling aperture stirring, and $20 \mathrm{MHz}$ frequency stirring. Measurements were performed at $1800 \mathrm{MHz}$, and one half-wave dipole was used as the DUT located at the centre of the chamber. The measured scenario " $A$ " is an empty chamber with a typical quasi-isotropic behaviour consisting of 50000 samples. The quasi-isotropic behaviour of the measured scenario is reproduced in Fig. 45, wherein AS is defined as in [114].

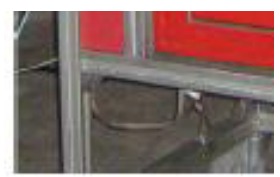

Figure 44. A two-cavity MSRC.

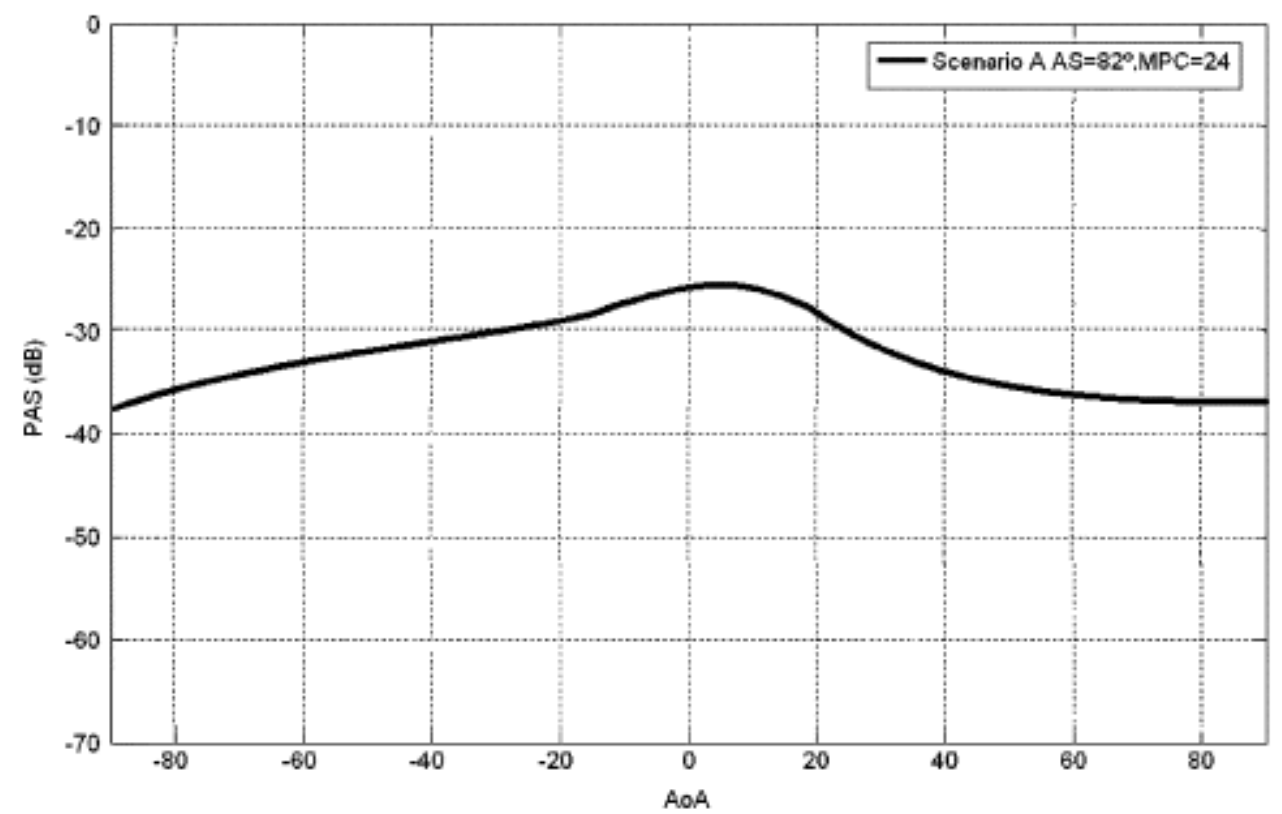

Figure 45. Histogram of measured AOA using the high resolution MUSIC algorithm for the initial Rayleigh fading measured scenario. 


\subsubsection{Sample Selection Technique Performance}

For comparison purposes, the performance analysis is performed by treating both a set of 10000 scalar ideal Rayleigh distributed data samples from theoretical formulas and the 50000 sample set measured at scenario " $A$ " as illustrated in Fig. 45. Five different Rician-fading target distributions were defined and are depicted in Fig. 46. The specific parameters for each distribution are also illustrated in Table 21. Three of the distributions have the same mean output power as the initial ideal Rayleigh-fading distribution. The other two have a larger and lesser output power, respectively.

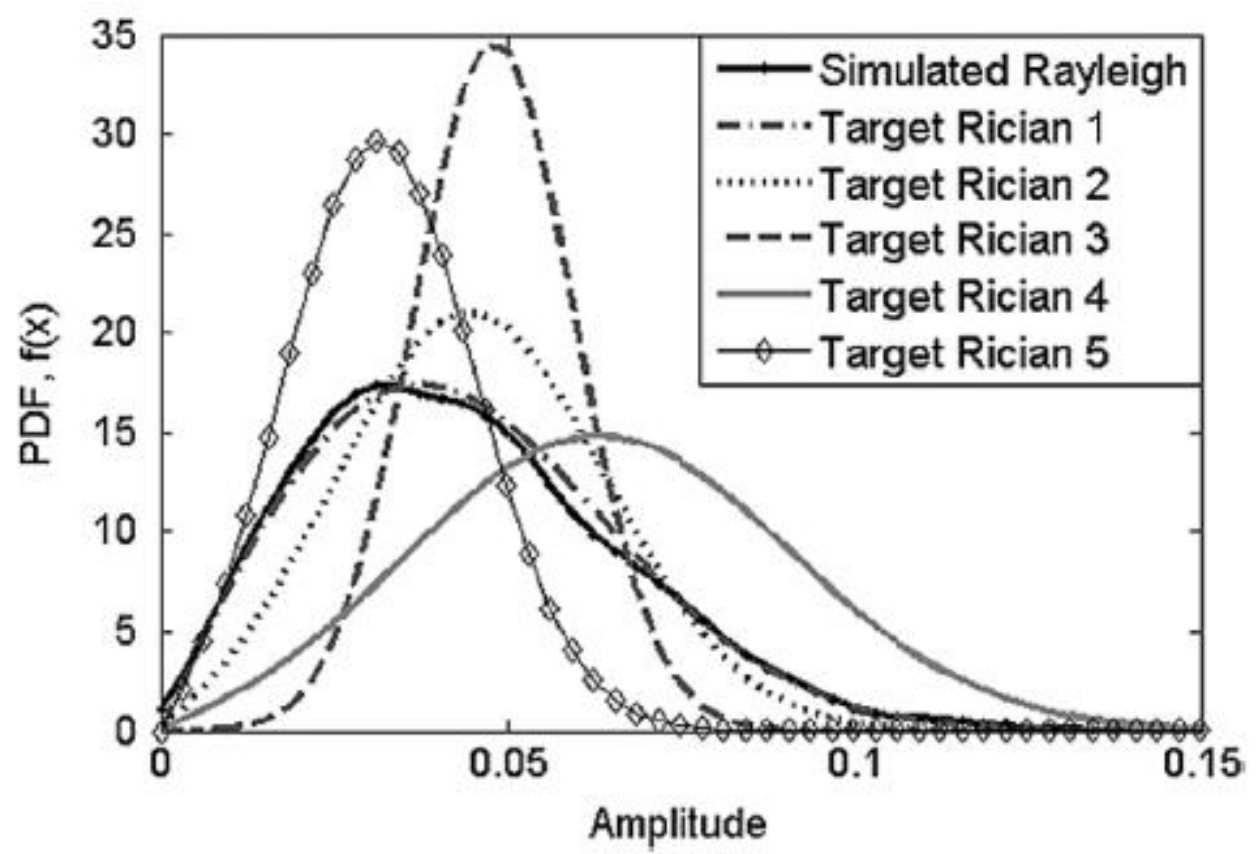

Figure 46. Initial (ideal theoretical Rayleigh) and target and target (ideal theoretical Rician) fading distributions.

TABLE 21. THE FIVE DIFFERENT TARGET RICE DISTRIBUtIONS

\begin{tabular}{c|cc|c|c}
\hline \hline $\begin{array}{c}\text { Target } \\
\text { Distribution }\end{array}$ & $a_{\text {target }}$ & $s_{\text {target }}$ & k-factor [linear] & $P_{\text {out }} / P_{\text {in }}$ \\
\hline Rice 1 & 0.0286 & 0.0286 & 0.5 & 1 \\
Rice 2 & 0.0404 & 0.0202 & 2 & 1 \\
Rice 3 & 0.0467 & 0.0117 & 8 & 1 \\
Rice 4 & 0.0572 & 0.0286 & 2 & 2 \\
Rice 5 & 0.0286 & 0.0143 & 2 & 0.5 \\
\hline \hline
\end{tabular}

The measured figures of merit are depicted in Table 22. From this table, it can be observed that very accurate Rician-fading emulation can be achieved with the sampleselection technique. It is also noticeable from this table that a large percentage of samples is discarded when distributions with larger mean output power than originally measured ones are targeted. The algorithm seems to discard more samples for those target distributions that have a larger output power than the initial Rayleigh-fading set, 
which poses a limitation to the technique. A comparison between Rician-fading target 4 and measured subset extracted using the sample-selection technique is illustrated in Fig. 47 as an example.

Table 22. Performance of Sample-Selection Technique With GA

\begin{tabular}{c|c|c|c|c}
\hline \hline Initial sample set & Target & Error [ $\left.\varepsilon_{d}\right]$ & $\begin{array}{c}\text { Discarded } \\
\text { samples [\%] }\end{array}$ & $\begin{array}{c}\text { Computation } \\
\text { time [s] }\end{array}$ \\
\hline Id. Rayleigh & Rician 1 & 0.0011 & 55.1 & 1.6 \\
Id. Rayleigh & Rician 2 & 0.0008 & 51.3 & 62 \\
Id. Rayleigh & Rician 3 & 0.0012 & 60.8 & 1168 \\
Id. Rayleigh & Rician 4 & 0.0005 & 70.3 & 1622 \\
Id. Rayleigh & Rician 5 & 0.0011 & 55.2 & 516 \\
Meas. Rayleigh & Rician 1 & 0.0013 & 50.7 & 102 \\
Meas. Rayleigh & Rician 2 & 0.0015 & 53.7 & 724 \\
Meas. Rayleigh & Rician 3 & 0.0024 & 67.2 & 5225 \\
Meas. Rayleigh & Rician 4 & 0.0011 & 75.5 & 7948 \\
Meas. Rayleigh & Rician 5 & 0.0021 & 54.6 & 1030 \\
\hline \hline
\end{tabular}

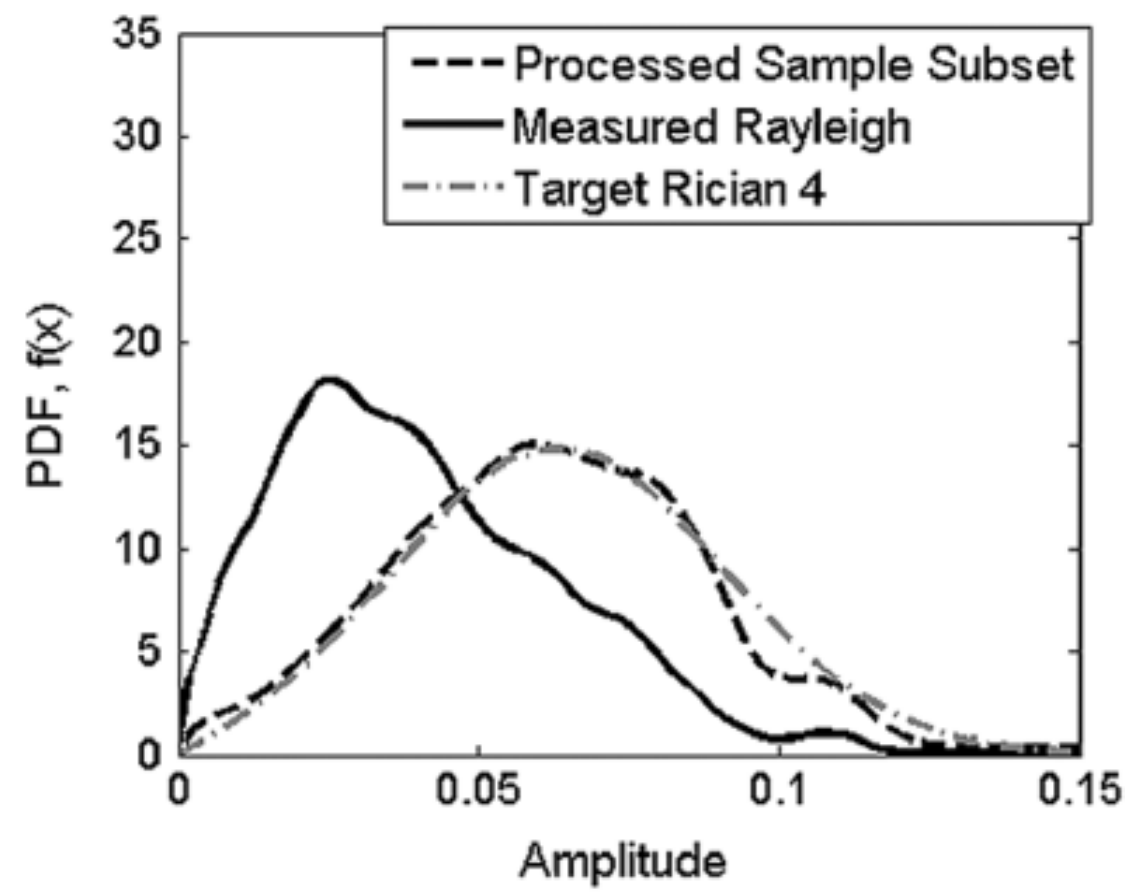

Figure 47. A comparison between initial measured Rayleigh-fading set, processed sample subset, and Rician-fading target 4.

It can also be observed from this figure that the use of large 50000 -samples measured Rayleigh-fading data makes the GA spend more time, which is to be expected due to the larger number of initial samples. Using both theoretical and measured Rayleigh-fading, initial sample sets provide very similar accuracies on final sample subsets. Likewise, it seems clear from the results that the higher the $k$-factor of the target Rician distribution, the higher the required computational time of the GA. 


\subsubsection{Sample-Selection Algorithms Performance}

In this section the performance of the three different algorithms used for the sample selection is validated by sample-selecting a set of 10000 simulated Rayleigh distributed scalar samples. The same five ideal Rician distributions defined in the previous section (see Table 21) are used as target distributions.

The performances of the three algorithms have been evaluated in respect of the distribution error and number of remaining samples due to changes of the $k$-factor. It is also of interest to see what happens with the number of remaining samples if the output power in the remaining samples $\left(P_{\text {out }}=a^{2}+2 s^{2}\right)$ is different from the input power of the initial samples.

In the results below, the fitness limit of the iterative GA is set to 0.01 and the hybrid is set to 0.001 . Figs. 48,49 and 50 show the behaviour of the algorithms when they are applied on the simulated data.

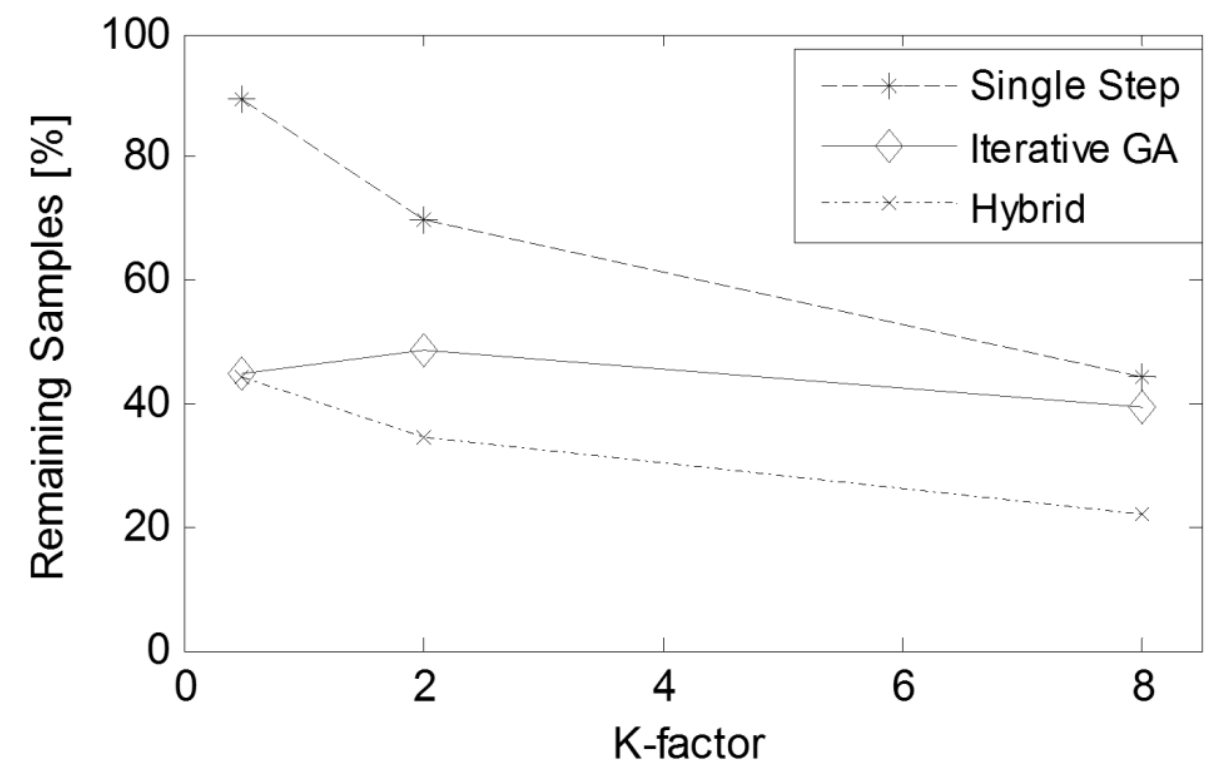

Figure 48. The amount of remaining samples for each of the algorithms vs. the $k$-factor. The target distribution of Rice $1(k=0.5)$, Rice $2(k=2)$ and Rice $3(k=8)$ with the same mean output power as the original distribution are indicated by points. The lines are added as guide for the eye. 


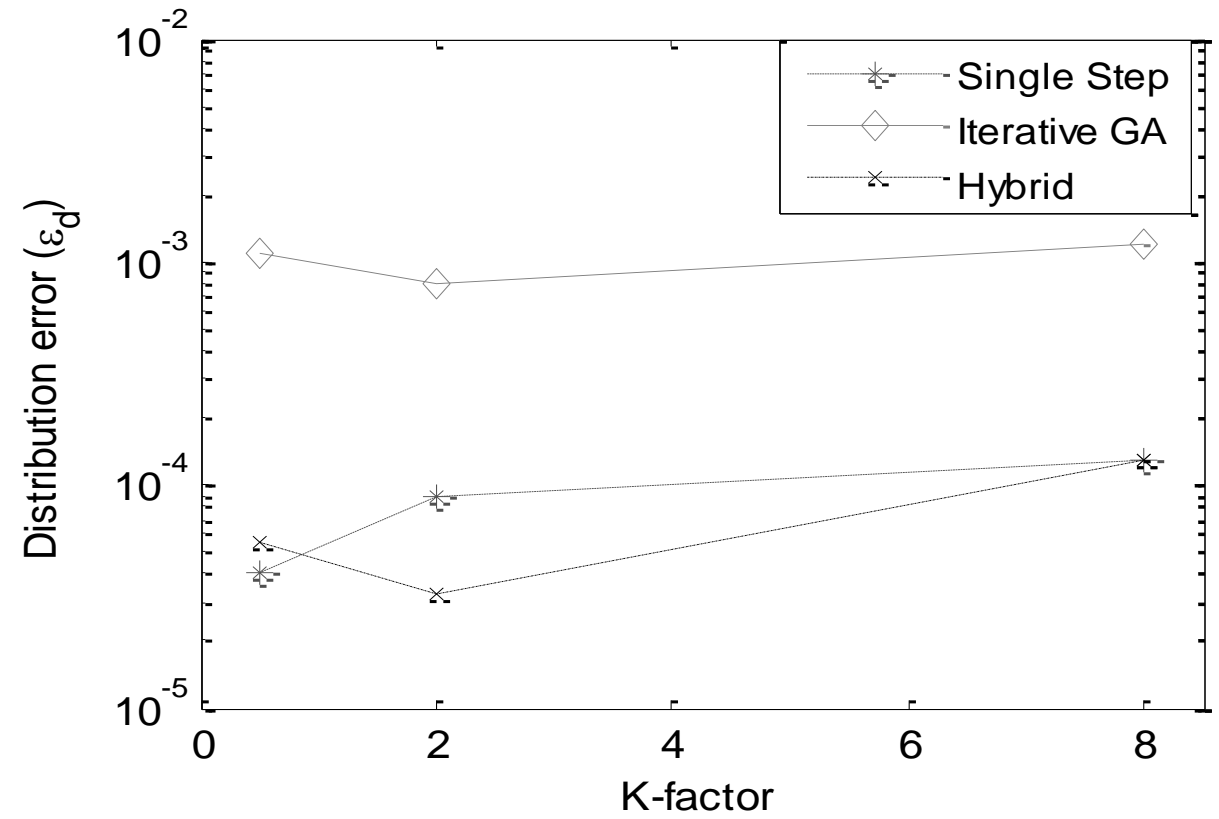

Figure 49. Distribution error of the three algorithms vs. the $k$-factor. The target distribution of Rice 1 $(k=0.5)$, Rice $2(k=2)$ and Rice $3(k=8)$ are indicated by points.

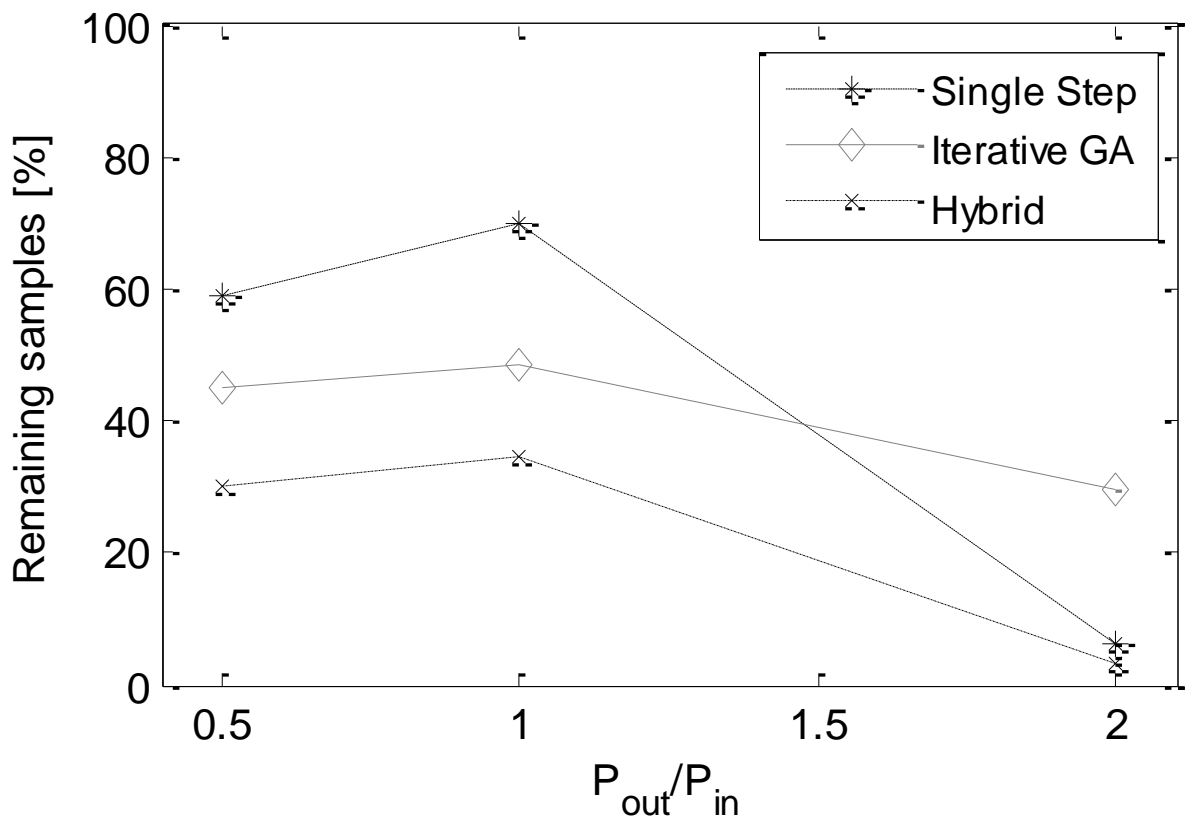

Figure 50. The ratio $P_{\text {out }} / P_{\text {in }}$ vs. amount of remaining samples for each algorithm. All three distributions have the same $k$-factor, $k=2$.

As a constraint of the algorithms, the target distribution should have the same mean power as the initial distribution. The reason for this is intuitively understood. If the output power is higher than the input power there will be a problem of finding data samples for the new distribution, also seen in Fig. 50. Similarly, if lower power relative to the power of the initial distribution is targeted, the same problem occurs. Thus, the algorithm should only be used to achieve the correct $k$-factor and shape of distribution, not as an amplifier or attenuator. 
Another point worth mentioning is the speed of the algorithms. The single step is extremely fast and computes all the final distributions within about 0.6 seconds, while the iterative GA within $2 \mathrm{~min}$ up to $50 \mathrm{~min}$, and the combination of these two, the Hybrid, ends up at with an average of 4.7 seconds

Sample selection is applied in this study in order to extend the capability of an MSRC from the emulated NLoS environment, to a simulated LoS environment. This feature makes it possible to extract the behaviour of a DUT in many different conditions, with a single measurement. This step up in the algorithm implementation makes the technique suitable for real-time applications, which is essential for the use of the technique in commercial applications. In this way, a calculation that will take up to one hour with the iterative GA can be solved within seconds with the Hybrid algorithm; furthermore it shows a much better accuracy than both the single step and the iterative GA.

In some cases it is seen that the hybrid algorithm took longer time than the iterative GA. This can be due to the first stop of the hybrid algorithm, the single step algorithm, filtered too many data samples for the iterative GA part of the Hybrid algorithm. With too few remaining samples in the second step, the iterative GA had problems finding an optimum solution within a limited time span. The effects on $N_{\text {ind }}$ and $\varepsilon_{\text {final }}$ of reducing the amount of remaining samples are overall very small.

\subsubsection{Validation of the technique}

In this section some practical results will be shown, in order to demonstrate the usefulness of this technique. As it has been said before, this technique does not try to resemble all the fading characteristics of a non-isotropic environment (like AoA, DS...), instead it is intended to demonstrate that it is possible to recover information about a passive antenna for different fading scenarios, just post-processing one isotropic measurement.

\subsubsection{Antenna Correlation and $D G$}

In this section it will be evaluated the application of the proposed sample selection technique to the antenna correlation and DG estimation for a given prototype implementing diversity (dual-antenna prototype). Prototypes $C$ and D defined in Chapter 5 are used in this study, both of them working at the $2.6 \mathrm{GHz}$ frequency band.

In order to validate the technique, the same measurements performed in Chapter 5 for the free space case are used as initial data in this section. That means, that a conventional MSRC is used, so the richness of the environment is very limited. However, as those prototypes have been already analyzed in Chapter 5 for some 
different LoS scenarios, it is interesting to apply the sample selection technique to them, in order to perform a similar study using this novel technique.

Departing from a free space measurement performed in a MSRC with a Rayleigh amplitude distribution, several Rician environments are set as target distributions with $k$-factors going from 1 to 10 in steps of 1 .

Fig. 51 shows the measured correlation for some different Rician $k$-factors. As it was seen in Chapter 5, when the isotropicity is reduced the high correlated prototype tend to improve its behaviour. On the contrary, the low correlated prototype shows a slightly increasing tendency of the measured correlation when the $k$-factor is increased.

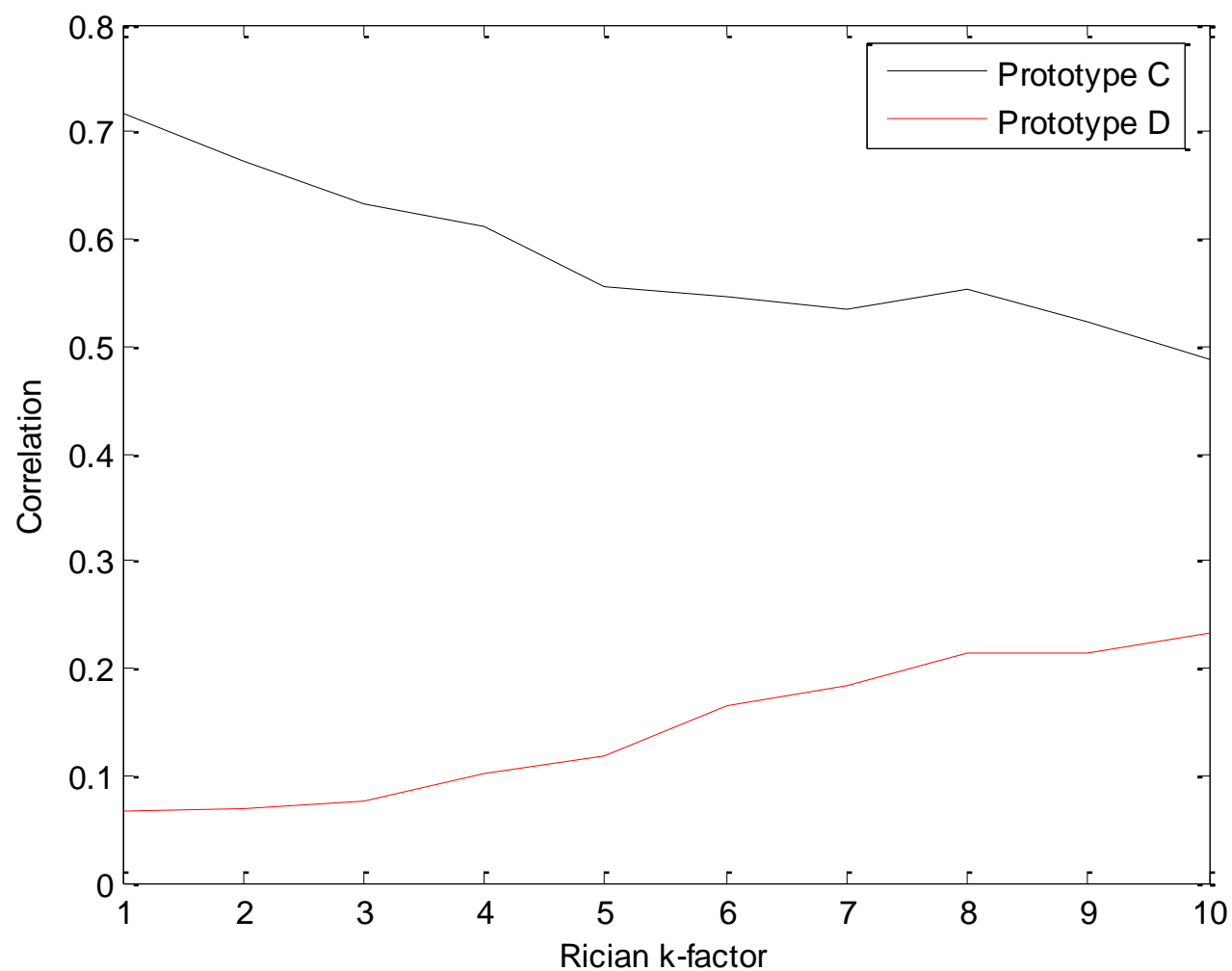

Figure 51. Correlation as a function of Rician $\boldsymbol{k}$-factor of a high correlated prototype (Prototypes $\mathrm{C}$ and D defined in Chapter 5).

Fig. 52 show the DG measured after the sample selection is applied to both prototypes. It can be appreciated that Prototype $C$ presents a slight decrease in its diversity performance when the LoS condition is higher, while for Prototype D DG the effect is much stronger for high $k$-factors. This results are in line when those obtained in with the offset technique (as seen in Fig. 41), where the effect of the LoS component in terms of DG is much more significant for low correlated antennas. 
Obtained results show also the same effect detected in chapter 5 , where the same prototypes where measured under some different user influence scenarios. DG becomes very similar for both prototypes, when the isotropicity of the environment is reduced. These results show the ability of this technique for the emulation using an MSRC of some non-isotropic effects, as those detected in chapter 5 for the user influence case. It is worth noticing here, that the user influence has other important effects over the antenna performance (specially reducing the mutual coupling between antennas), so not all those effects are emulated here, but just the effect of LoS created in the vicinity of the antennas when the environment is not isotropic.

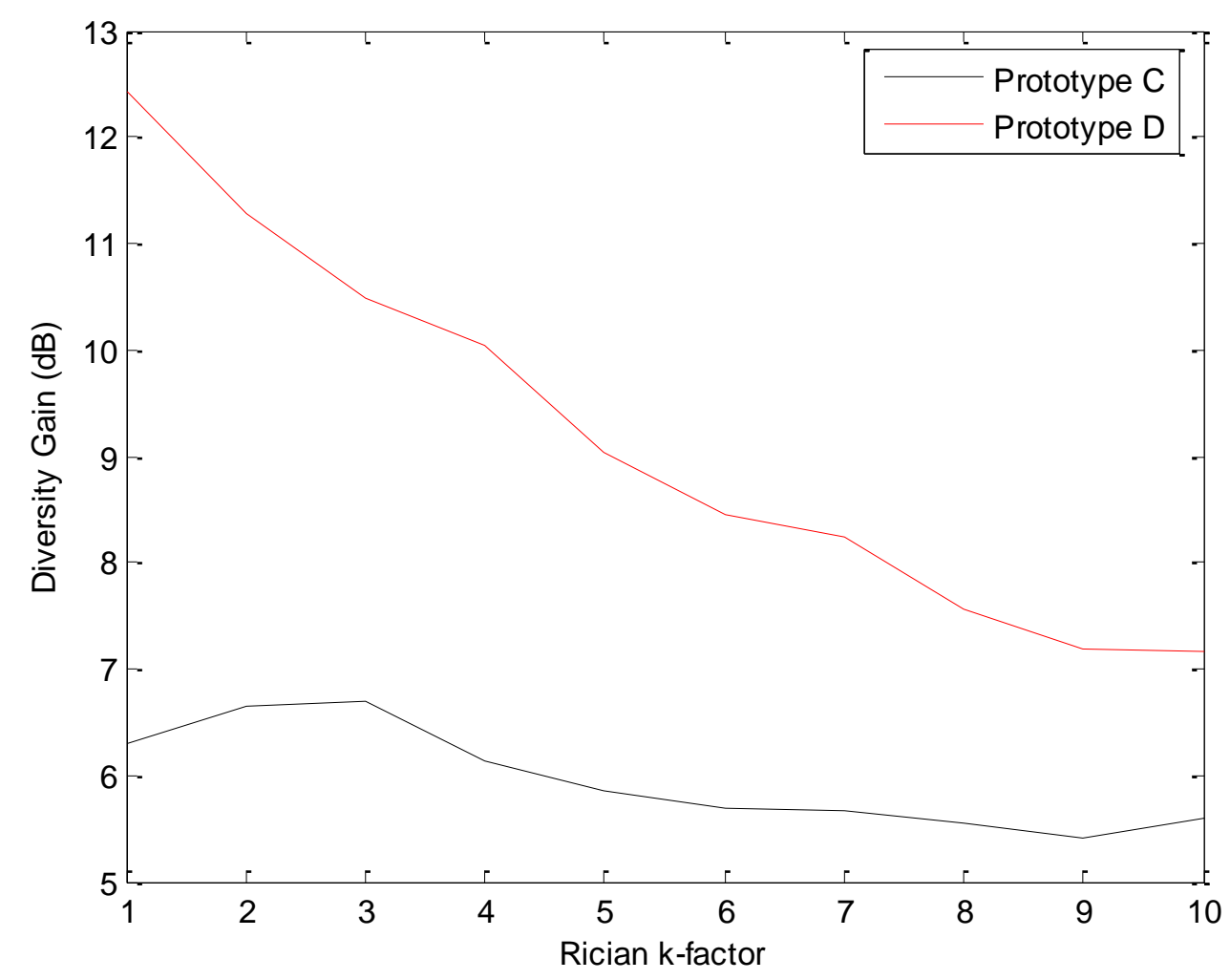

Figure 52. DG as a function of Rician $\boldsymbol{k}$-factor of a high correlated prototype (Prototype $C$ defined in Chapter 5).

\subsubsection{MIMO Capacity}

In this section, MIMO capacity is calculated for some Rician environments. To carry out this validation the same measurement performed in Section 7.2 (three dipoles in a vertical position with $f_{c}=1800 \mathrm{MHz}$ and $d=0.01 \lambda$ ), is used so results are comparable with those obtained in that section. Departing from one of the real measurements above, the sample selection was applied to that measurement taking as a target distributions three Rician channels with different $k$-factor. 
The simulated results were compared, in terms of MIMO Capacity, with the model for Rician environments described in [108]. Results are presented in Fig. 53. Since correlation between the antennas at the reception is high (three parallel dipoles, inside a MSRC), it is not expected to reach the maximum ergodic capabilities of the channel, as they are described in [108].

The results below show different values of MIMO Capacity calculated using both, the ideal model and the sample selection technique. As expected, the capacity of the real system is clearly below of the ideal case due to the high correlation in reception antennas.

It is especially interesting the result when the $k$-factor become very high $(k=49)$. As it is explained in [108], the correlation has a strong influence in MIMO Capacity, as long as the $k$-factor is relatively low. But when the LoS path become the most important part of the signal, then correlation have a low influence in the results of MIMO Capacity, and both lines simulated and theoretical become closer.

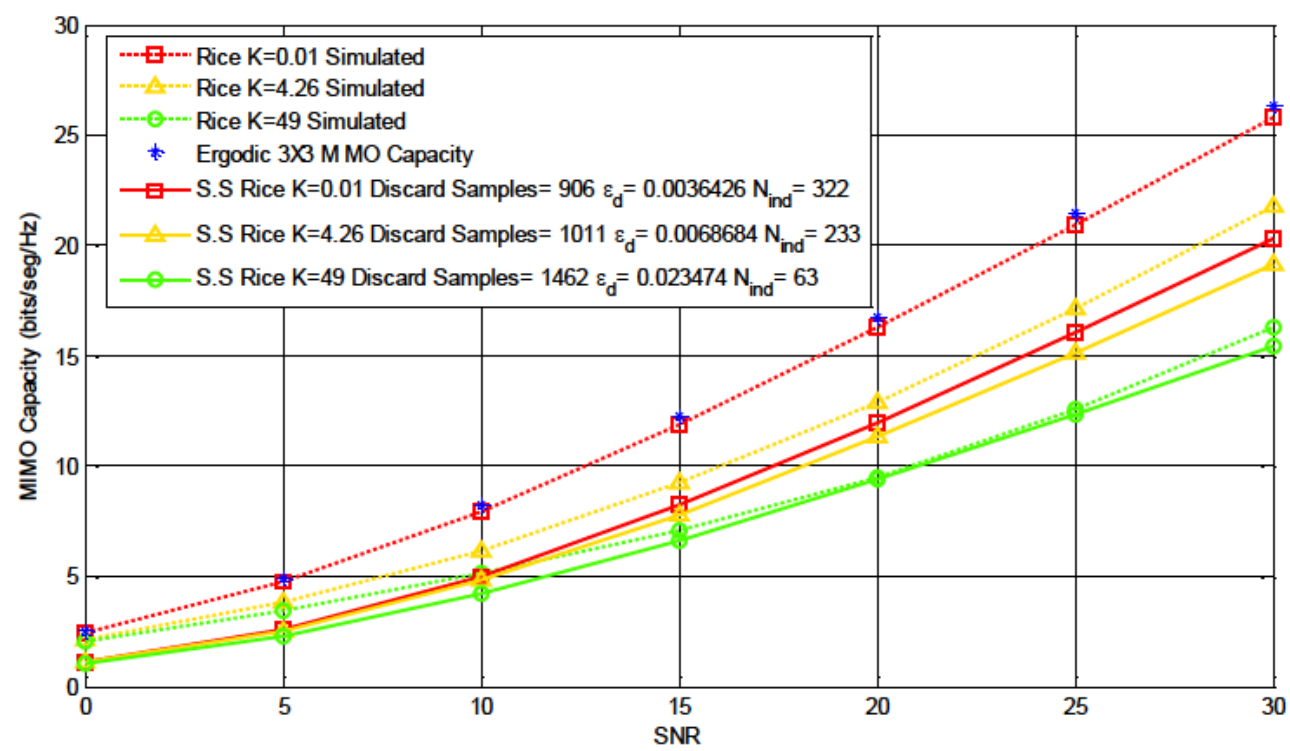

Figure 53. 3×3 MIMO capacity emulated using the simple selection technique with different Rician $k$ factors.

\subsubsection{Sample-Selection Application to Other Channel Metrics}

\subsubsection{Antenna Correlation}

One important parameter in MIMO OTA evaluation of wireless devices is correlation. Most carriers demand envelope correlation in wireless devices to remain below 0.5 for all operational frequencies and tested scenarios. Conventional MSRCs, however, can only test correlation for isotropic Rayleigh-fading scenarios. 
This section presents an extension of the sample selection technique, in which it is applied to the correlation coefficient between the received signals from two antennas, instead of applying it to the signal strength amplitude distribution. With this extension, novel enhanced MSRCs acquire versatile capabilities for using correlation as a MIMO OTA testing Figure of Merit (FoM) in a wide variety of testing scenarios. Applying the fundamental principle of the sample selection technique to the correlation coefficient improves its usefulness in tests of diversity and/or MIMO antennas, where the correlation coefficient is critical.

The aim of this section is to present the efficiency and accuracy of the sample selection technique applied to a different channel metric from the signal strength, as it is the antenna correlation (which depends on both the propagation channel, and the antenna design), and to demonstrate its practical usefulness with the application of the technique to some real prototypes.

Sample selection is here applied for the purpose of achieving a different correlation coefficient than the one at hand for the whole sequence, which represents that of an isotropic Rayleigh fading scenario. The correlation coefficient is calculated from dual channel measurements of the signals from a pair of antennas.

Dual channel data can be described by a bivariate (2-D) PDF, according to [65] [115],

$$
f\left(x_{1}, x_{2}\right)=\frac{x_{1} x_{2}}{\left(1-\rho^{2}\right) s_{1}^{2} s_{2}^{2}} \exp \left(-\frac{1}{2\left(1-\rho^{2}\right)} \cdot\left(\frac{x_{1}^{2}}{s_{1}^{2}}+\frac{x_{2}^{2}}{s_{2}^{2}}\right)\right) I_{0}\left(\frac{\rho x_{1} x_{2}}{\left(1-\rho^{2}\right) s_{1} s_{2}}\right)
$$

where $I_{0}$ is the modified Bessel function of the first kind and of order zero.

The parameters $s_{1}$ and $s_{2}$ in (83) define the magnitude on the two channels, respectively, and $\rho$ is the magnitude of the complex correlation coefficient between the data on the two channels, i.e. $\rho=\left|\rho_{c}\right|$.

Applying the sample selection technique to the two-dimensional data means that from the initial data, with its given PDF, a subset of samples is chosen to have a certain targeted PDF. In this process, the number of data samples is reduced. By mathematical derivation, we can calculate the maximum number of remaining samples for a given target correlation value, as a function of the correlation in the initial data by [116],

$$
\begin{gathered}
N_{t-}=\frac{1-\rho_{i}^{2}}{1-\rho_{t}^{2}} \bullet N_{i} \\
N_{t+}=\frac{\left(1+\rho_{i}\right) \bullet\left(1-\rho_{t}\right)}{\left(1-\rho_{i}\right) \bullet\left(1+\rho_{t}\right)} \bullet N_{i}
\end{gathered}
$$


where,

- $\rho_{i}$ is the correlation coefficient of the initial distribution.

- $\rho_{t}$ is the chosen target correlation.

- $N_{i}$ is the initial number of samples.

- $N_{t-}$ is the number of remaining samples, for the case where $\rho_{t}<\rho_{i}$.

- $N_{t+}$ is the number of remaining samples, for the case where $\rho_{t}>\rho_{i}$

The number of remaining samples is important in order to obtain a good accuracy in the measurements. In this case we can see a big reduction in the number of samples for some combinations of initial/target correlations. This knowledge is critical when implementing the sample selection technique and it must be taken into account when the algorithm is devised.

The accuracy with which the target correlation coefficient can be achieved is investigated through numerical simulations using Matlab. The individual branch distributions are first verified, with two randomly generated Rayleigh distributed sequences with $N_{i}=50000, s_{1 i}=1, s_{2 i}=2$, and $\rho_{i}=0.7$. The single-step algorithm is applied with maintained branch powers, i.e. $s_{1 t}=1$ and $s_{2 t}=2$, and with $\rho_{t}=0.2$.

Following this, the accuracy of the achieved correlation coefficient is investigated by performing sample selection on 500 realizations, and plotting the histogram PDF of the achieved correlation coefficients. This is done for two values of $\rho_{i}(0$ and 0.7$)$, and four values of $\rho_{t}(0.3,0.5,0.7$, and 0.9$)$, thus in total eight cases. Each case uses $N_{i}=10000, s_{1 i}=1$, and $s_{2 i}=2$. For comparison, in each case sequences of length $N_{t}$ and with target correlation coefficient $\rho_{t}$ are also generated directly, i.e. without any sample selection process. Due to the finite length of the sequences, these will also show some spread in the achieved correlation coefficient. Figs. 54 and 55 show PDFs of the achieved correlation coefficients, with histograms for the sample selection results, and solid lines for the directly generated data. The numbers next to the peaks are the $N_{t}$ values. Apparently, the accuracy depends on $\rho_{i}$ and $\rho_{t}$. Comparing the sample selection results with the directly generated data shows that the selection process itself does not increase the uncertainty by any significant amount, even though a small shift in the distribution is seen in Fig. 54 for $\rho_{t}=0.3$ and $\rho_{t}=0.9$. 


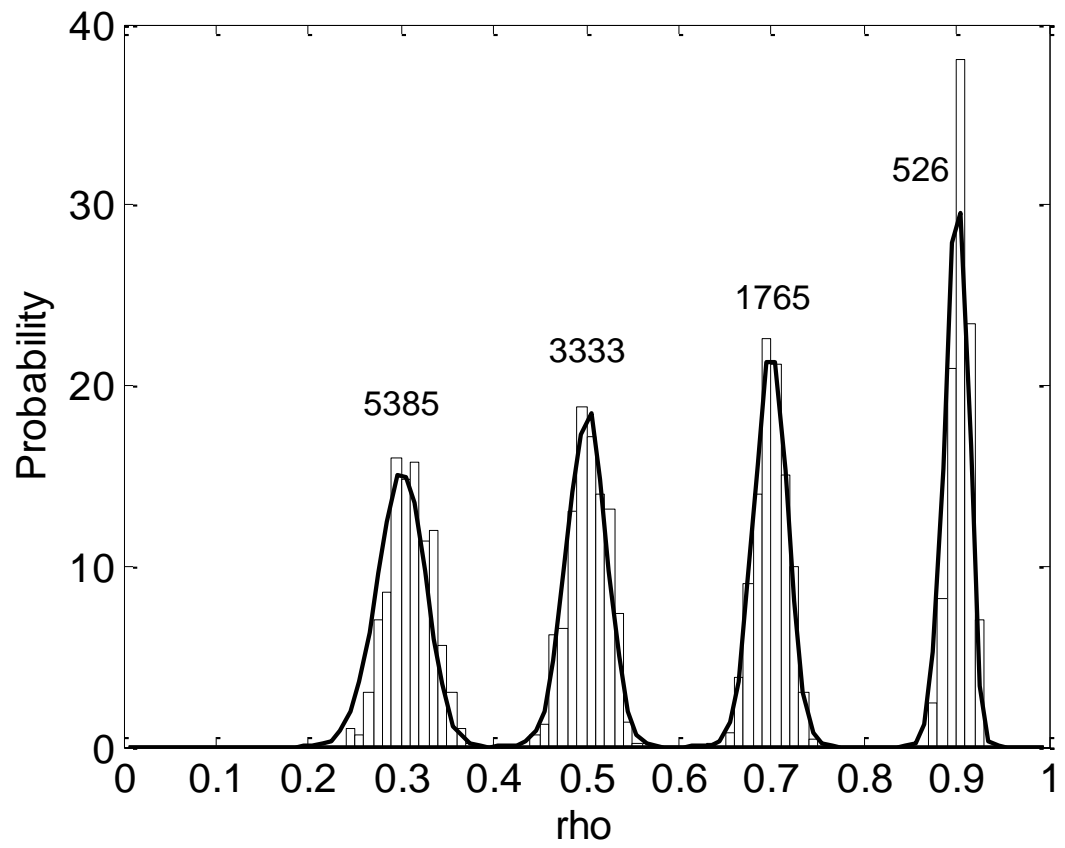

Figure 54. PDFs for $\rho_{i}=0$ and $\rho_{t}=0.3,0.5,0.7$, and $0.9 . N_{i}=10000$ in all cases. Numbers next to the peaks are $N_{t}$ values. Histograms are the results of simulations with $\mathbf{5 0 0}$ realizations. Solid lines are spreads due to finite sequence length for the $N_{t}$ values at hand.

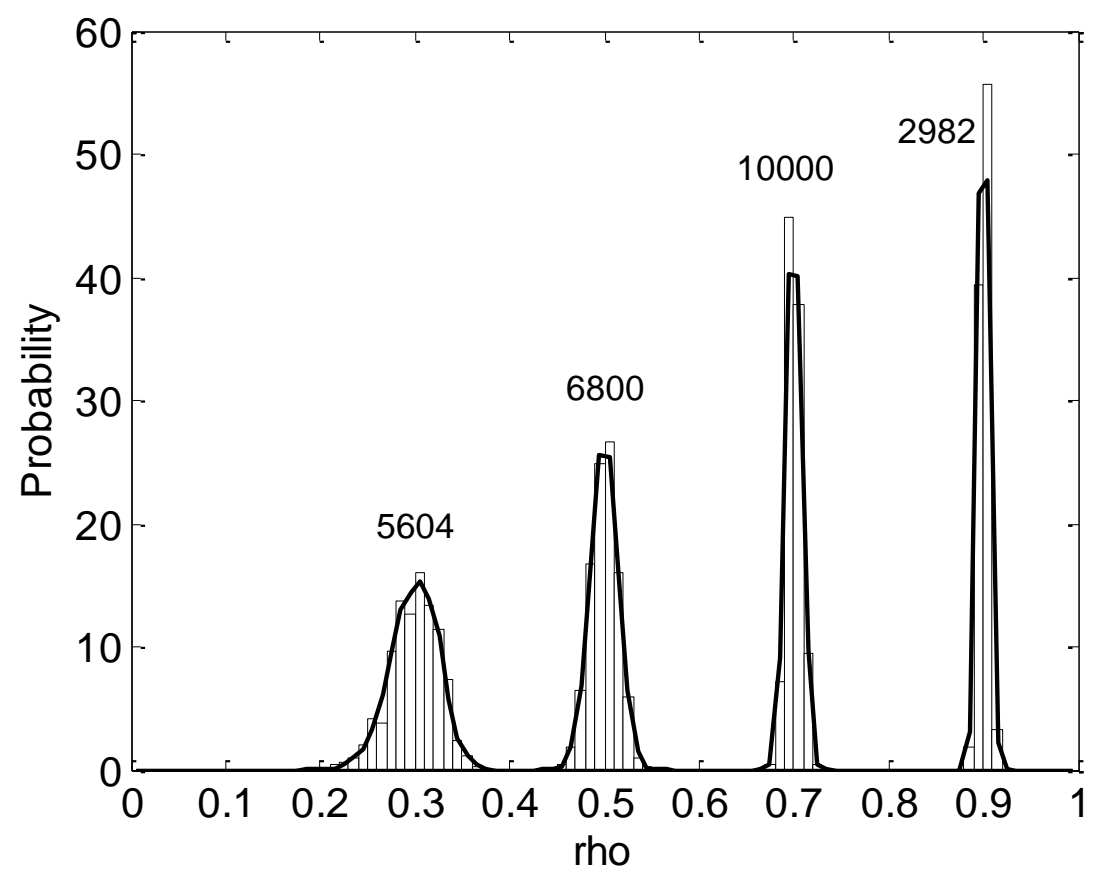

Figure 55. PDFs for $\rho_{i}=0.7$ and $\rho_{t}=0.3,0.5,0.7$, and $0.9 . N_{i}=10000$ in all cases. Numbers next to the peaks are $N_{t}$ values. Histograms are the results of simulations with $\mathbf{5 0 0}$ realizations. Solid lines are spreads due to finite sequence length for the $\boldsymbol{N}_{t}$ values at hand.

Varying $N_{i}$ shows that the spread of the achieved correlation coefficient follows the relation 


$$
\sigma_{\rho} \propto \frac{1}{\sqrt{N_{t}}}
$$

which means that the proportion of remaining samples is very critical for the accuracy.

When implementing the sample selection technique, an algorithm is used to automatically process the measured data. Several algorithms have been investigated in this chapter. In this section, the single step algorithm is used.

Measurements are carried out using an MSRC. The measurement frequency is 2600 $\mathrm{MHz}$ and 1601 points are taken $\left(N_{i}=1601\right)$. Complex transmission coefficients $\left(S_{21}\right)$ between the transmitting antenna in the MSRC and each one of the receiving antennas are measured using a Rohde \& Schwarz ZVRE Vector Network Analyzer. The data is stored as two simultaneous sequences of transmission coefficients with a certain length. These sequences constitute the initial data.

The ability of the implemented algorithm to select data sets with arbitrary correlation coefficient is clearly demonstrated. As an example, in Figs. 56 and 57, the sample selection algorithm is executed for the purpose of increasing the correlation from the initial value of $0.05\left(\rho_{i}=0.05\right)$ to a target value of 0.95 . Intermediate values are also taken in steps of 0.05 . There we can see in Fig. 56 the resulting values of DG for each correlation value. DG is calculated at the $10 \%$ probability level and using the MRC scheme [37] [73]. In Fig. 57.it is shown the final number of remaining samples.

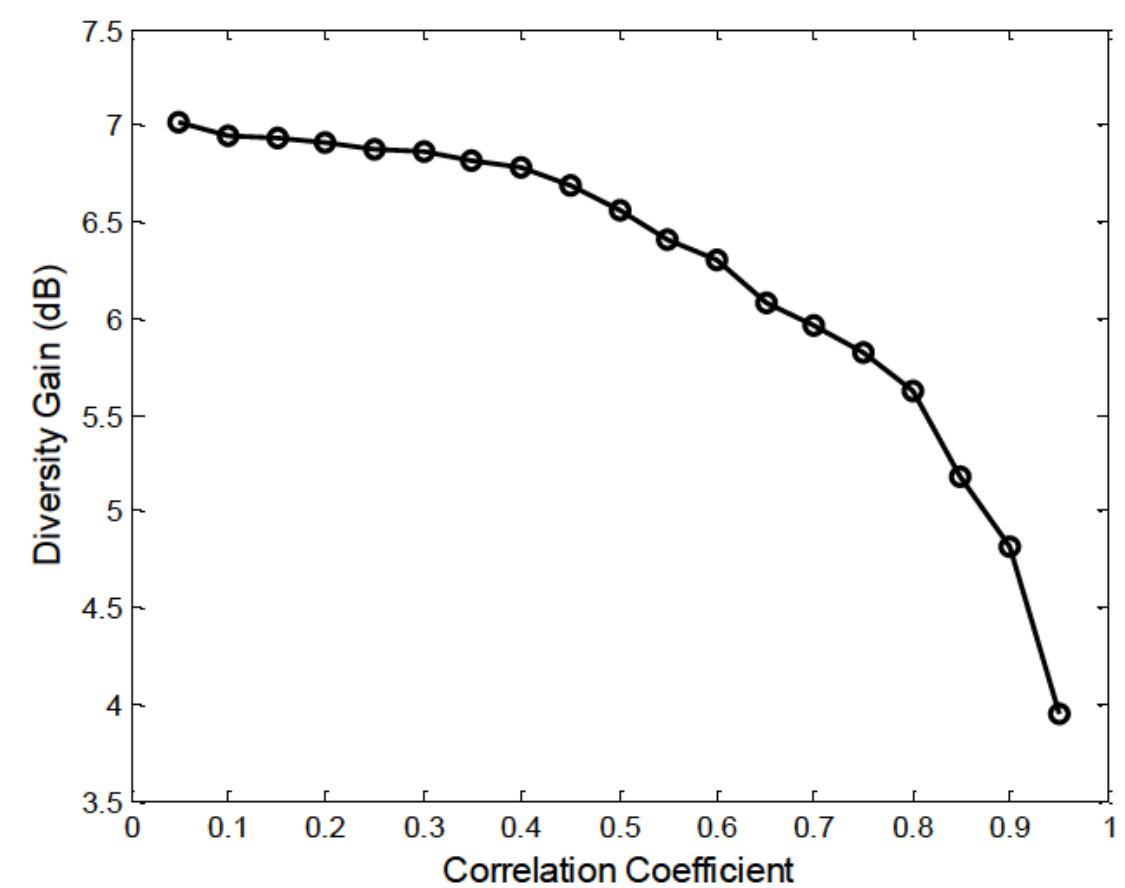

Figure 56. Diversity gain after sample selection, for the different values of target correlation coefficient. 


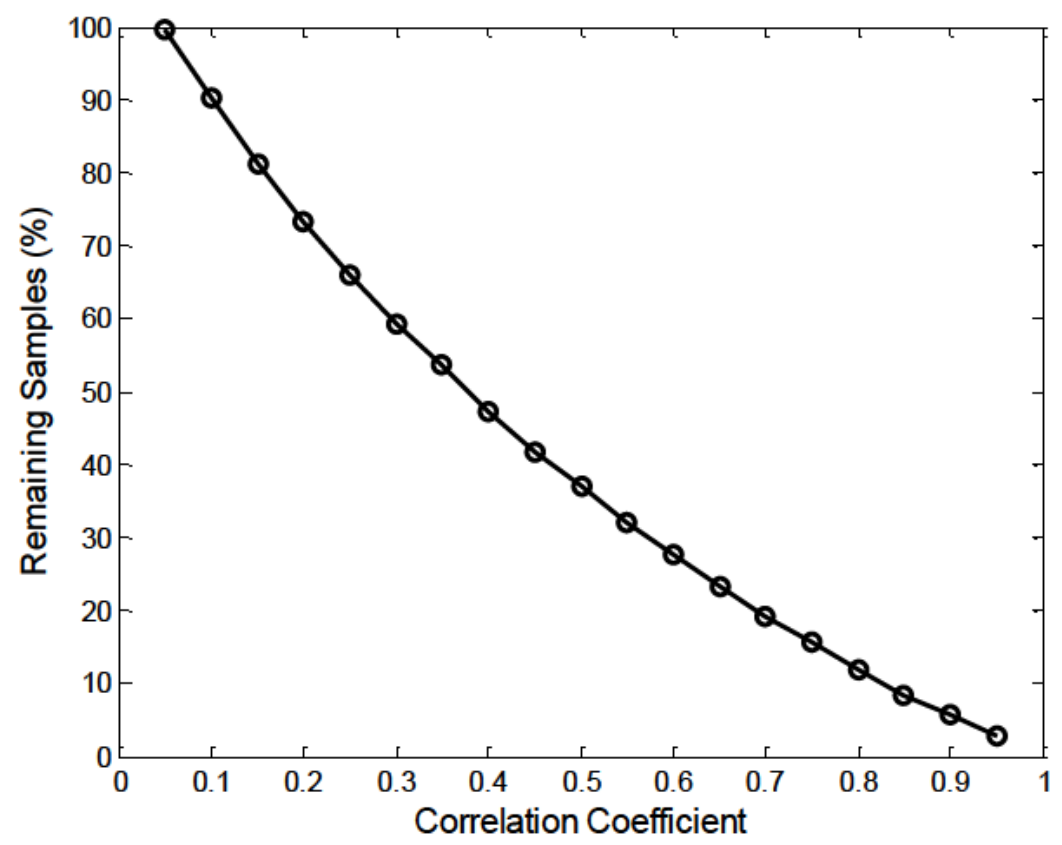

Figure 57. Number of remaining samples after sample selection, as a percentage of the number of initial samples $\left(\rho_{i}=0.05\right)$.

In this section we demonstrate the implementation of the sample selection technique to antenna correlation coefficient. The technique allows the user to obtain an ensemble of samples with any correlation coefficient within the interval $(0,1)$ for a given antenna pair. Measurements with real antennas are presented, demonstrating the usefulness of the technique to characterize antenna pairs for non-isotropic environments, where the correlation between antennas is different from this obtained under isotropic conditions.

\subsection{Conclusion}

In the first part of this chapter, an offset technique has been proposed for the accurate emulation of any Rician-fading environment a MSRC for the first time. The new technique allows for greater versatility and a more detailed study of the influence of the $k$-factor on MIMO performance. Correlation, diversity gain and MIMO capacity have been analyzed for three different MIMO systems under a variety of Rician-fading environments with different $k$-factors. Different effects have been evaluated. Results confirm previously-published influence of $k$-factors for outdoor measurements, yet this time with the use of a MSRC for the first time. The proposed technique allows for a better study of $k$-factor influence on MIMO performance for wireless communications systems, avoiding cumbersome outdoor measurements. The technique represents a step forward to the goal of obtaining real-world performance from a MSRC test.

With the sample-selection technique proposed in the second section of this chapter, it has been demonstrated the ability of MSRCs to accurately emulate arbitrary 
fading distributions. This has been done without any hardware alteration within the MSRC for the first time. The obtained data set is composed of unmodified measured samples conforming the desired fading statistical ensemble. The sample-selection technique can further enhance the capabilities of MSRCs for MIMO OTA measurements, closing the current gap between MSRCs and more expensive spatialfading emulators.

Three different algorithms have been successfully employed. This has been demonstrated with high accuracy using both theoretical data samples and measured data sets in an MSRC. The accuracy is adequate for most cases shown in the study but the cases also reveal the behaviour of the algorithms. If a large change of distribution is calculated fewer samples will be remaining and there will be a reduction of accuracy.

With the use of combined linear-ga, accurate simulation is obtained at low computation time. To be able to emulate a wireless channel some improvements has to be done in the future. For instance the angle of arrival (AoA) has to be emulated as well.

Validation of the technique has been provided, for passive measurements, agreeing very well with the correlation and DG values obtained in Chapter 5, as well as with the published model of the Rician Channels for MIMO Capacity [30]. In the last section, a different approach has been proposed for the sample selection procedure, so emulated propagation channel is defined in terms of the correlation experienced by the antennas, instead of the distribution of the signal strength. This approach opens the door to extend the sample selection technique for MSRCs to any other channel parameter that is interesting for the antenna designer.

The sample-selection technique can further enhance the capabilities of modestirred chambers for and with some more research be used in MIMO OTA measurements. Future research also includes the emulation of a wide range of standardised channel models for $4 \mathrm{G}$ terminal research, development and compliance testing. 


\section{Conclusion}

\subsection{Conclusion and Future Research}

In this thesis there have been analyzed several characteristics that are of interest for $4 \mathrm{G}$ mobile phone designers. The study has been based on the effect that non-ideal environments have on various metrics that characterize the behaviour of multiantenna devices. One of these factors is the effect that the user presence causes on the mobile terminal performance. This effect has been extensively studied for the case of single antenna terminals. However, in terminals that use MIMO technology, nearly as important as the effect on the antenna itself is the effect on the propagation environment of the signal that causes anisotropy in the vicinity of the antenna and may diminish many of the ideal benefits of multi-antenna technology. This is due mainly to the fact that the ergodic capacity proposed for MIMO systems assume the existence of a very rich propagation environment where you can set different parallel communication channels uncorrelated with each other.

As a consequence of that, it is important to clearly separate the effect that the user has over the antenna and over the environment. To this end, this thesis has also analyzed the effect that the number of signals used to emulate the propagation channel have on the parameters that define a multi-antenna device. This study reveals that, in conditions where the number of signals is very small, the devices do not exhibit behaviour similar to that presented in rich environments, and devices that have operations in isotropic conditions clearly recognizable as good or bad, do not exhibit these behaviours when the richness of the environment decreases.

But all these factors have a crucial dependence on the measurement technique on which measurements are made. Various techniques exist for OTA analysis of mobile devices, although three are fundamental: anechoic chamber based, mode-stirred chamber based and 2-stage method. 
One of the techniques that has received most attention in recent years has been the MSRC technique. This technique has a number of advantages over the others, such as measurement speed in three dimensions, and their low cost compared to other alternatives. However, the fact that the environment generated in the interior of the MSRC is naturally isotropic and distributed according to a Rayleigh FDP has always limited the use of this technique to measurements under isotropic environments and multi-reflective channel models (typically urban environments).

In this thesis two software processing techniques have been proposed to circumvent this inherent difficulty of the MSRC. With the first technique called "offset technique", it is possible to extend the measurement capabilities of the MSRC so that any passive device can be evaluated in a Rician distributed environment with an arbitrary $k$-factor. Other techniques, based on hardware modifications had been previously proposed for this purpose. However, with the "offset technique" the emulation is done precisely with a $k$-factor fully controllable, and does not involve any modification that disturbs the reverberant environment of the MSRC resonant cavity or cavities. The limitation of this technique is that it cannot be extended to active devices such as mobile phones.

To overcome this drawback, a second processing software alternative has been proposed in this thesis, called "sample selection technique". With this technique, given a measure in the Rayleigh environment naturally generated by the MSRC, it is possible to get the device behaviour in arbitrary fading environment. For this, the procedure followed consists in selecting only part of the samples measured along the entire sequence of stirring of the MSRC, in accordance with a criterion imposed on selected samples. This criterion is variable, and this thesis has focused the analysis on the statistical distribution of the amplitude of the received field. By this criterion, different environments such as Rician, Nakagami or Log-Normal, can be easily emulated in an MSRC. However, this technique has the limitation that if the MSRC does not provide a very rich environment, it may be difficult to obtain the desired distribution patterns. Therefore, this technique is intended to be applied to second-generation MSRC, which incorporate multiple resonant cavities and provide a greater wealth in the measured field distribution.

The future continuation of the research work performed in this thesis involves the development and thorough evaluation of the mechanisms by which the user's presence produces environments with a limited number of signals, and therefore highly anisotropic environments. Through the detailed study of these effects, we intend to incorporate them into existing channel models, so that the OTA 
measurements made in laboratories resemble the most to the actual behaviour that the user experiences in real environments.

To this end, advances in software processing will be proposed for basic MSRCs. Because the ultimate goal of OTA characterization of devices is the characterization of complete assets (phones, laptops, dongles ...), also an important part of future work will focus on the implementation and validation of the sample selection technique for active FoMs such as data throughput, by comparison with other measurement techniques OTA established, as data throughput is the most sought FoM both by manufacturer and for operators to discern between good and bad systems.

Thus the work developed in this thesis represents only a first step towards better understanding of the propagation factors that determine the behaviour of real multiantenna systems, such as those for next mobile generation (4G). This understanding is fundamental in developing appropriate measurement techniques to emulate the different propagation environments the mobile phone industry demands. This is why the evolution of OTA measurement techniques in general, and MSRCs in particular represent a field of research where there is still much to discover in the coming years.

\subsection{Results Obtained as a Result of this Thesis}

As a result of this thesis, seven articles have been accepted for publication in different international journals. Three more, have been submitted, and by the time of this writing, are still under reviewing process. Three more articles have been published in national journals, and seven contributions have been made to international conferences. Below there is a detailed enumeration of these results.

\subsubsection{International Journals}

I. Sanchez-Heredia, J.D.; Gruden, M.; Valenzuela-Valdes, J.F.; Sanchez-Hernandez, D.A.; , "SampleSelection Method for Arbitrary Fading Emulation Using Mode-Stirred Chambers," Antennas and Wireless Propagation Letters, IEEE , vol.9, no., pp.409-412, 2010.

doi: 10.1109/LAWP.2010.2049729

URL:

http://ieeexplore.ieee.org/stamp/stamp.jsp?tp=\&arnumber=5460965\&isnumber=5423326

II. Sanchez-Heredia, J.D.; Valenzuela-Valdes, J.F.; Martinez-Gonzalez, A.M.; Sanchez-Hernandez, D.A.; , "Emulation of MIMO Rician-Fading Environments With Mode-Stirred Reverberation Chambers," Antennas and Propagation, IEEE Transactions on , vol.59, no.2, pp.654-660, Feb. 2011

doi: 10.1109/TAP.2010.2096185

URL:

http://ieeexplore.ieee.org/stamp/stamp.jsp?tp=\&arnumber $=5654569$ \&isnumber $=5705613$ 
III. Sánchez-Heredia, J.D.; Hallbjörner, P.; Bolin, T.; Martínez-González, A.M.;, “HSDPA Throughput Performance with Limited Number of Signal Paths" Antennas and Wireless Propagation Letters, IEEE, vol.11,no., pp. 484-487, 2012. doi: 10.1109/LAWP.2012.2197591 URL: http://ieeexplore.ieee.org+stamp.jsp?tp=\&arnumber=6193406

IV. Sánchez-Heredia, J.D.; Hallbjörner, P.; Valenzuela-Valdés, J.F.; Bolin, T.; Martínez-González, A.M.;, "Different User influence on MIMO Handset Antenna Performance in Reverberation Chamber" Microwave and Optical Technology Letters. Submitted. February 2012.

V. Sánchez-Heredia, J.D.; Hallbjörner, P.; Bolin, T.; Martínez-González, A.M.;, “Lab Measurements of HSDPA Performance with Realistic Channel Conditions and User Influence Including Real Person" Wireless Communications, IEEE Transactions on. Submitted. December 2011

VI. Garcia-Fernandez, M.A.; Sanchez-Heredia, J.D.; Martinez-Gonzalez, A.M.; Sanchez-Hernandez, D.A.; Valenzuela-Valdes, J.F.; , "Advances in mode-stirred reverberation chambers for wireless communication performance evaluation," Communications Magazine, IEEE , vol.49, no.7, pp.140-147, July 2011

doi: 10.1109/MCOM.2011.5936167

URL:

http://ieeexplore.ieee.org/stamp/stamp.jsp?tp=\&arnumber=5936167\&isnumber=5936142

VII. Hallbjörner, P.; Sanchez-Heredia, J. D.; Lindberg, P.; Martinez-Gonzalez, A. M.; Bolin, T.; , "Multipath Simulator Measurements of Handset Dual Antenna Performance With Limited Number of Signal Paths," Antennas and Propagation, IEEE Transactions on , vol.60, no.2, pp.682-688, Feb. 2012

doi: 10.1109/TAP.2011.2173451

URL:

http://ieeexplore.ieee.org/stamp/stamp.jsp?tp=\&arnumber=6060879\&isnumber=6142627

VIII. Hallbjörner, P., Sánchez-Heredia, J. D., de los Reyes, E. and Sánchez-Hernández, D. A. (2011), Limit for the proportion of remaining samples in the mode-stirred chamber sample selection technique. Microwave and Optical Technology Letters, 53: 2608-2610. doi: 10.1002/mop.26336

IX. Hallbjörner, P.; Sánchez-Heredia, J.D.; Martinez Gonzalez, A.M.;, "Mode-Stirred Chamber Sample Selection Technique Applied to Antenna Correlation Coefficient". International Journal of Antennas and Propagation. Accepted for publication. January 2012.

URL: http://www.hindawi.com/journals/ijap/aip/986514/

X. Marín-Soler, A.; Grudén, M.; Sánchez-Heredia, J.D.; Hallbjörner, P.; Martínez-González, A.M.; Rydberg, A.; Sánchez-Hernández, D.A.;, "Sample Selection Algorithms for Enhanced MIMO Antenna Measurements using Mode-Stirred Reverberation Chambers" Antennas and Propagation, IEEE Transactions on. Accepted for publication. February 2012.

\subsubsection{National Journals}

I. Sánchez Heredia, J.D.; García Fernández, M.A.; Valenzuela Valdés, J.F.; Martínez González, A.M.; Sánchez Hernández, D.A.; "Estudio de la presencia del usuario en cámara de reverberación" Jornadas de introducción a la investigación de la UPCT, ISSN 1888-8356, no. 3, 2010 , pp. 65-67 
II. García Fernández, M.A.; Sánchez Heredia, J.D.; Valenzuela Valdés, J.F.; Martínez González, A.M.; Sánchez Hernández, D.A.; "Distancia Natural de Acoplamiento de una Cabeza Humana a una Antena en la Banda C de WiMAX" Jornadas de introducción a la investigación de la UPCT, ISSN 1888-8356, no. 3, 2010 , pp. 62-64

III. Sánchez-Heredia, J.D.; Beltrán-Alfageme, D.; Valenzuela Valdés, J.F.; García-Fernández, M.A.; Martínez González, A.M.; , "MIMO TIS/TRP active testing with second-generation mode-stirred chambers" Espacio-Teleco: revista de la ETSI-UPCT, ISSN 2171-2042, no. 2, 2011 , pp. 123-132

\subsubsection{International Conferences}

I. Sanchez-Heredia, J.D.; Garcia-Fernandez, M.A.; Gruden, M.; Hallbjörner, P.; Rydberg, A.; Sanchez-Hernandez, D.A.; , "Arbitrary fading emulation using mode-stirred reverberation chambers with stochastic sample handling," Antennas and Propagation (EUCAP), Proceedings of the 5th European Conference on , vol., no., pp.152-154, 11-15 April 2011

URL:

http://ieeexplore.ieee.org/stamp/stamp.jsp?tp=\&arnumber=5781805\&isnumber=5780481

II. Sánchez-Heredia, J.D.; Valenzuela-Valdés, J.F.; Hidalgo, J.P.; Torrecilla, A.; Lobato, S.; SánchezHernández, D.A.; , "Evaluation of MIMO OTA parameters for LTE using a Mode-Stirred Reverberation Chamber" COST 2100. TD(10)12073. Bologna, Italy. 23-25 Nov 2010

III. Sanchez-Heredia, Juan D.; Hallbjörner, Paul; Bolin, Thomas;, "Multipath Simulator Measurements of Throughput with Different Angular Distributions of Delay" COST IC1004. TD(11)01004. Lund, Sweden. 21-22 Jun 2011

IV. Sanchez-Heredia, Juan D.; Hallbjörner, Paul; Bolin, Thomas;, "Throughput Comparison of the Same Set of Phones in Multipath Simulator and Reverberation Chamber" COST IC1004. TD(11)01004. Lund, Sweden. 21-22 Jun 2011

V. Sanchez-Heredia, Juan D.; Marín-Soler, Adoración; Hallbjörner, Paul; Martínez-González, Antonio M.; Sanchez-Hernandez, David A.; , "User Influence Over LTE Band XII Handset Performance" 6th European Conference on Antennas and Propagation, EuCAP 2012. Accepted. Prague, Czech Republic. 26-30 March 2012

VI. Valenzuela-Valdes, Juan F.; Sanchez-Heredia, Juan D.; Garcia-Fernandez, Miguel A.; , "True polarization diversity for non-isotropic scenarios," Antennas and Propagation (EuCAP), 2010 Proceedings of the Fourth European Conference on , vol., no., pp.1-4, 12-16 April 2010 URL:

http://ieeexplore.ieee.org/stamp/stamp.jsp?tp=\&arnumber=5505505\&isnumber=5504894

VII. Hallbjörner, Paul; Sanchez-Heredia, Juan D.; Martínez-González, Antonio M.; Marín-Soler, Adoración; Sanchez-Hernandez, David A.; , "Versatile Emulation of Antenna Correlation Coefficient as MIMO OTA Figure of Merit Using Mode-Stirred Reverberation Chambers" 6th European Conference on Antennas and Propagation, EuCAP 2012. Accepted. Prague, Czech Republic. 26-30 March 2012 


\section{References}

[1] Directive 2004/108/EC, "'On the approximation of the laws of the Member States relating to electromagnetic compatibility and repealing Directive 89/336/EEC," in Official Journal of the European Union, 2004, pp. 1-14.

[2] Europeean Committee for Electrotechnical Standardization CENELEC, "Product standard to demonstrate the compliance of mobile phones with the basic restrictions related to human exposure to electromagnetic fields ( $300 \mathrm{MHz}-3 \mathrm{GHz}$ )," in EN 50360, 2001.

[3] European Committee for Electrotechnical Standardization CENELEC, "Basic Standard for the Measurement of Specific Absorption Rate Related to Human Exposure to Electromagnetic Fields from Mobile Phones (300 MHz - $3 \mathrm{GHz}$ )," in EN 50361, 2001.

[4] International Electrotechnical Commission IEC, "Human exposure to radio frequency fields from hand-held and body-mounted wireless communication devices - Human models, instrumentation, and procedures - Part 1: Procedure to determine the specific absorption rate (SAR) for hand-held devices used in close," in IEC 62209, 2005.

[5] International Association for the Wireless Communications CTIA, "Method of Measurement for Radiated RF Power and ," in Test Plan for Mobile Station Over the Air Performance, 2011.

[6] 3rd Generation Partnership Project 3GPP, "User Equipment (UE) / Mobile Station (MS) Over The Air (OTA) antenna performance; Conformance testing (Release 8)," in TS 34.114. Technical Specification Group Radio Access Network, 2010.

[7] Juan F. Valenzuela-Valdés, Emulación de entornos multicamino y estudio de parámetros que limitan los sistemas MIMO empleando cámara de reverberación. Cartagena: PhD Thesis, Universidad Politécnica de Cartagena, 2008.

[8] 3rd Generation Partnership Project 3GPP, "Measurement of radiated performance for MIMO and multi-antenna reception for HSPA and LTE terminals (Release 11)," TR 37.976, Technical Specification Group Radio Access Networks;

[9] Ya Jing, Zhu Wen, Hongwei Kong, S. Duffy, and M. Rumney, "Two-stage over the air (OTA) test method for MIMO device performance evaluation," in Antennas and Propagation (APSURSI), 2011 IEEE International Symposium on , 3-8 July 2011, pp. 71-74.

[10] 3rd Generation Partnership Project 3GPP, "Spatial channel model for Multiple Input Multiple Output (MIMO) simulations (Release 9)," TR 25.996, Technical Specification Group Radio Access Network 2009.

[11] D.S. Baum, J. Hansen, and J. Salo, "An interim channel model for beyond-3G systems: extending the 3GPP spatial channel model (SCM)," in Vehicular Technology Conference, 2005. VTC 2005Spring. 2005 IEEE 61st , 30 May-1 June 2005 , pp. 3132 - 3136 Vol. 5.

[12] H. El-Sallabi, et al. IST-WINNER D5.4 D.S. Baum, "Final report on link and system level channel models," ver 1.4. Oct.2005.

[13] Kyösti et al., "WINNER II Channel Models," IST-WINNER D1.1.2 p., ver 1.1, Sept. 2007.

[14] International Electrotechnical Commission IEC, "Electromagnetic Compatibility (EMC) Part 4-21: Testing and Measurement Techniques - Reverberation Chamber Test Methods," in IEC Standard 61000-4-21, 2003.

[15] P. Corona and et al., "Performance and analysis of a reverberating enclosure with a variable geometry," IEEE Trans. on Electromagnetic Compatibility, vol. 22, no. 1, pp. 2-5, 1980.

[16] M.A. García-Fernández and D. Sánchez-Hernández, "Emulación del perfil de potencia-retardo de modelos de canal espacial extendido en cámara de reverberación de modos agitados," IV Jornadas de introducción a la investigación de la UPCT, vol. 4, no. ISSN: 1888-8356, pp. 103-105, mayo 2011.

[17] P-S. Kildal, K. Rosengren, J. Byun, and J. Lee, "Definition of effective chamber," Microwave and Optical Technology Letters, vol. 34, no. 1, pp. 56-59, 2002.

[18] P.S. Kildal, C. Carlsson, and J. Yang, "Measurement of free space impedances of small antennas in reverberation chambers," Microwave and Optical Technology Letters, vol. 32, no. 2, pp. 112-115, January 2002.

[19] C.S. Lee, A. Duffy, and C. Lee, "Antenna Efficiency Measurements in a Reverberation Chamber 
Without the Need for a Reference Antenna," Antennas and Wireless Propagation Letters, IEEE, vol. 7, pp. 448-450, 2008.

[20] K. Rosengren and P.S. Kildal, "Correlation and Capacity of MIMO Systems and Mutual Coupling, Radiation Efficiency, and Diversity Gain of Their Antennas: Simulations and Measurement in a Reverberation Chamber," IEEE Communications Magazine, pp. 104-112, Dec 2004.

[21] J.F. Valenzuela-Valdés, A.M. Martínez-González, and D. Sánchez-Hernández, "Effect of User Presence on Receive Diversity and MIMO Capacity for Rayleigh-Fading Channels," IEEE Antennas and Wireless Propagation Letters, vol. 6, pp. 596-599, 2007.

[22] J.F. Valenzuela-Valdés, A.M. Martínez-González, and D. Sánchez-Hernández, "Emulation of MIMO non-isotropic fading environments with reverberation chambers," IEEE Antennas and Wireless Propagation Letters, vol. 7, pp. 325-328, 2008.

[23] D.A. Hill, J.M. Ladbury, P.F. Wilson, G. Koepke, and J. Coder, C.L. Holloway, "On the Use of Reverberation Chambers to Simulate a Rician Radio Environment for the Testing of Wireless Devices," IEEE Transactions on Antennas and Propagation, vol. 54, no. 11, pp. 3167-3177, Nov. 2006.

[24] A. Cozza and A.E.-B.A. El-Aileh, "Accurate Radiation-Pattern Measurements in a Time-Reversal Electromagnetic Chamber," Antennas and Propagation Magazine, IEEE, vol. 52, no. 2, pp. 186-193, April 2010.

[25] U. Carlberg, P.S. Kildal, A. Wolgang, O. Sotoudeh, and C. Orlenius, "Calculated and Measured Absorption Cross Sections of Lossy Objects in Reverberation Chamber," IEEE Transactions on Electromagnetic Compatibility, vol. 46, no. 2, pp. 146-154, May 2004.

[26] N. Serafimov, P-S. Kildal, and T. Bolin, "Comparison between radiation efficiencies of phone antennas and radiated power of mobile phones measured in anechoic chambers and reverberation chamber," Antennas and Propagation Society International Symposium, IEEE, vol. 2, pp. 478-481, 2002.

[27] C. Orlenius, P.-S. Kildal, and G., Poilasne, "Measurements of total isotropic sensitivity and average fading sensitivity of CDMA phones in reverberation chamber," in IEEE Antennas and Propagation Society International Symposium, July 3-8 July, 2005, pp. 409-412.

[28] J. Clegg et al., "Optimization of Stirrer Designs in a Reverberation Chamber," IEEE Trans. Electromagnetic Compatibility, vol. 47, pp. 824-832, Nov. 2005.

[29] D.A. Hill, "Electromagnetic Theory of Reverberation Chambers," NIST Technical Note No.1506, Dec.1998.

[30] L. Young, and E. M. T. Jones G. Matthaei, Microwave Filters, Impedance-Matching Networks, and Coupling Structure. Boston, MA: Artech House, 1980.

[31] T. A. Loughry and S. H. Gurbaxani, "The Effects of Intrinsic Test Fixture Isolation on Material Shielding Effectiveness Measurements Using Nested Mode-Stirred Chambers," IEEE Trans. Electromagnetic Compatibility, vol. 37, no. 3, pp. 449-452, 1995.

[32] P. Plaza-Gonzalez et al., "New Approach for the Prediction of the Electric Field Distribution in Multimode Microwave-Heating Applicators with Mode Stirrers," IEEE Trans. Magnetics, vol. 40, no. 3, pp. 1672-1678, May 2004.

[33] P. Corona, G. Ferrera, and M. Migliaccio, "Reverberating Chambers as Sources of Stochastic Electromagnetic Fields," IEEE Trans. On Electromagnetic Compatibility, vol. 38, no. 3, pp. 348-356, 1996.

[34] K.A. Remley, S.J. Floris, H.A. Shah, and C.L. Holloway, "Static and Dynamic Propagation-Channel Impairments in Reverberation Chambers," IEEE Tran. on Electromagnetic Compatibility, vol. 53, no. 3, pp. 589-599, 2011.

[35] M. Kang and M.-S. Alouini, "Impact of correlation on the capacity of MIMO channels," Proc. IEEE Int. Conf. Communications, pp. 2623-2627, May 2003.

[36] D. A. Hill and J. M. Ladbury, "Spatial correlation functions of fields and energy density in a reverberation chamber," IEEE Trans. Electromagn. Compat., vol. 44, pp. 95-101, Feb 2002.

[37] R. G. Vaughan and J. B. Andersen, "Antenna diversity in mobile communications," IEEE Trans. Veh. Technol, vol. VT-36, pp. 147-172, Nov 1987. 
[38] P.-S. Kildal, K. Rosengren, J. Byun, and J. Lee, "Definition of effective diversity gain and how to measure it in a reverberation chamber," Microwave and Optical Technology Letters, vol. 34, pp. 56-59, 2002.

[39] E. Telatar, "Capacity of Multi-antenna Gaussian Channels," Transactions on Emerging Telecommunications Technologies, vol. 10, pp. 585-595, 1999.

[40] S. O. Rice, "Statistical properties of a sine wave plus random noise," Bell Syst. Tech. J., vol. 27, pp. 109-157, Jan. 1948.

[41] J. D. Sánchez-Heredia et al., "Evaluation of MIMO OTA parameters for LTE using Mode-Stirred Reverberation Chamber," in COST 2100 - TD(10)12073, Bologna, Italy, 2010, pp. 1-8.

[42] A. Sorrentino et al., "The Reverberating Chamber as a Line-of-Sight Wireless Channel Emulator," IEEE Trans. Antennas and Propagation, vol. 56, no. 6, pp. 1825-1830, June 2008.

[43] J. Frolik et al., "A Compact Reverberation Chamber for Hyper-Rayleigh Channel Emulation," IEEE Trans. Antennas and Propagation, vol. 57, no. 12, Dec. 2009.

[44] J. S. Hong, "Multimode Chamber Excited by an Array of Antennas," Electronics Letters, vol. 22, no. 19, pp. 1679-1680, 1993.

[45] D. Weinzierl et al., "Numerical Evaluation of Noncanonical Reverberation Chamber Configurations," IEEE Trans. Magnetics, vol. 44, no. 6, pp. 1458-1461., June 2008.

[46] Y. Huang and D. L. Edwards, "An Investigation of Electromagnetic Fields Inside A Moving Wall Mode-Stirred Chamber," in Proc. 8th IET Int'l. Conf. Electromagnetic Compatibility, 1992, pp. 115119.

[47] F. B. J. Leferink, "High Field Strength in A Large Volume: The Intrinsic Reverberation Chamber," in Proc. IEEE Int'l. Symp. Electromagnetic Compatibility, 1998, pp. 24-27.

[48] J. F. Valenzuela-Valdés et al., "Diversity Gain and MIMO Capacity for Non-Isotropic Environments Using A Reverberation Chamber," IEEE Antennas and Wireless Propagation Letters, vol. 8, pp. 112 115, 2009.

[49] E. Genender et al., "Simulating the Multipath Channel with A Reverberation Chamber: Application to Bit Error Rate measurements," IEEE Trans. Electromagnetic Compatibility, 2010.

[50] A. Sorrentino et al., "Characterization of NLOS Wireless Propagation Channels with A Proper Coherence Time Value in A Continuous Mode Stirred Reverberating Chamber," in Proc. 2nd European Wireless Technology Conf., 2009, pp. 168-171.

[51] A. Sorrentino et al., "On the Coherence Time Control of A Continuous Mode Stirred Reverberating Chamber," IEEE Trans. Antennas and Propagation, vol. 57, no. 10, pp. 3372-3374, Oct. 2009.

[52] M. Lienard and P. Degauque, "Simulation of Dual Array Multipath Channels Using Mode-Stirred Reverberation Chambers," Electronics Letters, vol. 40, no. 10, pp. 578-580, 2004.

[53] O. Delangre et al., "Modeling in-Vehicle Wideband Wireless Channels Using Reverberation Chamber Theory," in Proc. IEEE Vehic. Tech. Conf., Sept. 2007, pp. 2149-2153.

[54] Z. Yun and M. F. Iskander, "MIMO Capacity for Realistic Wireless Communications Environments," in Proc. IEEE Antennas and Propagation Society Int'l. Symp., June 2004, pp. 1231-1234.

[55] H. Fielitz et al., "Reverberation-Chamber Test Environment for Outdoor Urban Wireless Propagation Studies," IEEE Antennas and Wireless Propagation Letters, vol. 9, pp. 52-56, 2010.

[56] Robert W. Stewart and Ian A. Glover Faisal Darbari, MIMO Channel Modelling, Signal Processing, Available from: http://www.intechopen.com/articles/show/title/mimo-channel-modelling ed., ISBN: 978-953-7619-91-6 Sebastian Miron (Ed.), Ed.: InTech, 2010.

[57] P. Almers et al., "Survey of channel and radio propagation models for wireless mimo systems," EURASIP Journal on Wireless Communications and Networking, Volume 2007.

[58] L. Schumacher, K. I. Pedersen, P. E. Mogensen, and F. Frederiksen, J. P. Kermoal, "A stochastic MIMO radio channel model with experimental validation," IEEE Journal on Selected Areas in Communications, vol. 20, no. 6, pp. 1211-1226, 2002.

[59] W. Weichselberger, M. Herdin, H. Ozcelik, and E. Bonek, "A stochastic MIMO channel model with joint correlation of both link ends," IEEE Transactions on Wireless Communications, vol. 5, no. 1, pp. 90-99, 2006. 
[60] K. Bakowski and K. Wesolowski, "Change the Channel ," Vehicular Technology Magazine, IEEE, vol. 6, no. 2, pp. 82-91, June 2011.

[61] A. Saleh and R. A. Valenzuela, "A statistical model for indoor multipath propagation," IEEE J. Select. Areas Commun., vol. SAC-5, no. 2, pp. 128-137, Feb 1987.

[62] COST 231, "Urban transmission loss models for mobile radio in the 900 - and $1800 \mathrm{MHz}$ bands," TD(90)119 Rev. 2, Sep 1991.

[63] L. J. Greenstein, S. Ghassemzadeh, V. Erceg, and D. G. Michelson, "Ricean K-factors in narrowband fixed wireless channels," Proc.WPMC'99, Amsterdam, The Netherlands, Sep 1999.

[64] Andreas F. Molisch, Wireless Communciations, 2nd ed.: John Wiley and Sons, 2011.

[65] M. Nakagami, "The m-distribution-A general formula of intensity distribution of rapid fading," Statistical Methods in Radio Wave Propagation, pp. 3-36, 1960.

[66] A. Fort and et al, "Ultra-Wideband Channel Model for Communication Around the Human Body," IEEE Journal on Selected Areas in Communications, vol. 24, no. 4, pp. 927-933, April 2006.

[67] A. Fort, C. Desset, P. De Doncker, P. Wambacq, and L. Van Biesen, "'An ultra-wideband body area propagation channel Model-from statistics to implementation," Microwave Theory and Techniques, IEEE Transactions on, vol. 54, no. 4, pp. 1820-1826, June 2006.

[68] S. Skjaerris and G. Pedersen, "Influence on antenna diversity for a handheld phone by the presence of a person," Proc. IEEE Veh. Technol. Conf., 1997.

[69] T. Zervos et al., "The influence of MIMO terminal user's hand on channel capacity," in The first European Conference on Antennas and Propagation (EUCAP 2006), Nice, France, 6-10 Nov 2006.

[70] C. Gomez-Calero, N. Jamaly, L. Gonzalez, and R. Martinez, "Effect of mutual coupling and human body on MIMO performances," in EUCAP 2009. 3rd European Conference on Antennas and Propagation, 23-27 March 2009, pp. pp.1042-1046.

[71] F. Harrysson, J. Medbo, A. Molisch, A. Johansson, and F. Tufvesson, "Efficient experimental evaluation of a MIMO handset with user influence," Wireless Communications, IEEE Transactions on, vol. 9, no. 2, pp. 853-863, Feb. 2010.

[72] V. Plicanic, Buon Kiong Lau, A. Derneryd, and Zhinong Ying, "Actual Diversity Performance of a Multiband Diversity Antenna With Hand and Head Effects," Antennas and Propagation, IEEE Transactions on, vol. 57, no. 5, pp. 1547-1556, May 2009.

[73] D. G. Brennan, "Linear diversity combining techniques," Proc. IRE, vol. 47, pp. 1075-1102, June 1959.

[74] P.-S. Kildal and K. Rosengren, "Electromagnetic analysis of effective and apparent diversity gain of two parallel dipoles," Antennas and Wireless Propagation Letters, IEEE, vol. 2, pp. 9-13, 2003.

[75] J. F. Valenzuela-Valdes, M. A. Garcia-Fernandez, A. M. Martinez-Gonzalez, and D. SanchezHernandez, "The Role of Polarization Diversity for MIMO Systems Under Rayleigh-Fading Environments," Antennas and Wireless Propagation Letters, IEEE, vol. 5, no. 1, pp. 534-536, Dec 2006.

[76] M. Harteneck, M. Boloorian, S. Georgoulis, and R. Tanner, "Throughput measurements of HSDPA 14 Mbit/s terminal," Electronics Letters, vol. 41, no. 7, pp. 425-427, March 2005.

[77] H. Holma and J. Reunanen, "3GPP Release 5 HSDPA Measurements," in Personal, Indoor and Mobile Radio Communications, 2006 IEEE 17th International Symposium on, Sept. March 2006, pp. 1-5.

[78] C. Mehlführer, S. Caban, and M. Rupp, "Measurement-Based Performance Evaluation of MIMO HSDPA," Vehicular Technology, IEEE Transactions on, vol. 59, no. 9, pp. 4354-4367, Nov. 2010.

[79] Tao Cui et al., "Throughput Optimization in High Speed Downlink Packet Access (HSDPA)," Wireless Communications, IEEE Transactions on, vol. 10, no. 2, pp. 474-483, Feb. 2011.

[80] V. Plicanic and B.K. Lau, "Impact of spacing and gain imbalance between two dipoles on HSPA throughput performance," Electronics Letters, vol. 45, no. 21, pp. 1063-1065, October 2009.

[81] Chung-Huan Li, E. Ofli, N. Chavannes, and N. Kuster, "Effects of Hand Phantom on Mobile Phone Antenna Performance," Antennas and Propagation, IEEE Transactions on, vol. 57, no. 9, pp. 27632770, Sept. 2009. 
[82] S.L. Cotton and W.G. Scanlon, "An experimental investigation into the influence of user state and environment on fading characteristics in wireless body area networks at $2.45 \mathrm{GHz}$," Wireless Communications, IEEE Transactions on, vol. 8, no. 1, pp. 6-12, Jan 2009.

[83] T. Sakata et al., "BER Evaluation System for a Handset Antenna in a Multipath Environment Using a Spatial Fading Emulator," in International Symposium on Antennas and Propagation, ISAP, Seoul, Korea, 2005, pp. 351-354.

[84] A. Yamamoto et al., "Effectiveness of a fading emulator in evaluating the performance of MIMO systems by comparison with a propagation test," in Antennas and Propagation (EuCAP), 2010. Proceedings of the Fourth European Conference on, Barcelona, Spain, 12-16 April, 2010, pp. 1-5.

[85] P. Hallbjörner, Z. Ying, M. Håkansson, T. Anttila, and J. Welinder, "Bringing the Mobile Terminal Drive Test Into the Lab," in Proc. RVK08, Växjö, Sweden, 9-11 June 2008, pp. 94-98.

[86] P. Hallbjörner et al., "Multipath Simulator for Mobile Terminal Antenna Characterisation," IET Microwaves, Antennas and Propagation, vol. 4, no. 6, pp. 743-750, 2010.

[87] Xiang Gao, Buon Kiong Lau, Xiaoguang Wang, and T. Bolin, "On simplifying WINNER II channel model for MIMO OTA performance evaluation," in Antennas and Propagation (EUCAP), Proceedings of the 5th European Conference on, Rome, Italy, 11-15 April 2011, pp. 2942-2946.

[88] J.D. Sanchez-Heredia, P. Hallbjörner, and T. Bolin, "Multipath Simulator Measurements of Throughput with Different Angular Distributions of Delay," in COST IC1004, Tech. Rep. TD(11)01004, Lund, Sweden, June 2011.

[89] 3rd Generation Partnership Project 3GPP, "TS 25.101, Technical Specification Group Radio Access Network; User Equipment (UE) radio transmission and reception (FDD) (Release 10)," Tech. Rep. [Online]. Available: http://www.3gpp.org.

[90] CTTC - EMITE Ing., "HSDPA SIMO OTA Round Robin Test Report," 3GPP RAN4 \#57, Jacksonville, USA, Tech. Rep. R4-104350 Nov. 2010.

[91] CTTC - EMITE Ing., "HSDPA SIMO OTA Round Robin Comparison Tests including NIST Indoor-Urban and EPA Channel Models," 3GPP RAN4 \#57, Jacksonville, USA, Tech. Rep. R4-104839 Nov. 2010.

[92] SATIMO Industries, "MIMO OTA Round Robin Testing Campaign: SATIMO Testing Results," 3GPP RAN4 AH\#4, Xi'an, China, Tech. Rep. R4-103844 Oct. 2010.

[93] Nokia, "MIMO OTA Measurement Campaign," 3GPP RAN4 \#56, Madrid, Spain, Tech. Rep. R4103846 Aug. 2010.

[94] M. J. Gans, "A Power-Spectral Theory of Propagation in the Mobile-Radio Environment," IEEE Transactions on Vehicular Technology, vol. VT-21, no. 1, pp. 27-38, February 1972.

[95] P. L. Carro, J. de Mingo, and P. G. Ducar, "Analysis of the Antenna Stochastic Effective Gain in Mobile Environments," in IEEE 69th Vehicular Technology Conference, VTC Spring 2009, Barcelona, Spain, 26-29 April, 2009.

[96] K. Kalliola, "Experimental Analysis of Multidimensional Radio Channels," Espoo, Finland, PhD Thesis, Helsinki University of Technology Radio Laboratory Publications, Report S 251 February 2002.

[97] A. M. Sayeed and V. Raghavan, "Maximizing MIMO Capacity in Sparse Multipath With Reconfigurable Antenna Arrays," IEEE Journal of Selected Topics in Signal Processing, vol. 1, no. 1, pp. 156-166, June 2007.

[98] V. Raghavan, G. Hariharan, and A.M. Sayeed, "Capacity of Sparse Multipath Channels in the UltraWideband Regime," Selected Topics in Signal Processing, IEEE Journal of, vol. 1, no. 3, pp. 357-371, Oct. 2007.

[99] Xiyu Lu et al., "UWB-based Wireless Body Area Networks channel modeling and performance evaluation," in Wireless Communications and Mobile Computing Conference (IWCMC), 2011 7th International, 4-8 July 2011, pp. 1929-1934.

[100] M. Matthaiou, A.M. Sayeed, and J.A. Nossek, "Sparse multipath MIMO channels: Performance implications based on measurement data," in Signal Processing Advances in Wireless Communications, 2009. SPAWC '09. IEEE 10th Workshop on, June 2009, pp. 364-368.

[101] S. Saario, D. V. Thiel, J. W. Lu, and S. G. O'Keefe, "An Assessment of Cable Radiation Effects on Mobile Communications Antenna Measurements," in IEEE Antennas and Propagation Society 
International Symposium 1997, Montreal, Canada, 13-18 July 1997, pp. Vol. 1, 550-553.

[102] C. Icheln, J. Krogerus, and P. Vainikainen, "Use of Balun Chokes in Small-Antenna Radiation Measurements," IEEE Transactions on Instrumentation and Measurement, vol. 53, no. 2, pp. 498506, April 2004.

[103] P. Hallbjorner, J.D. Sanchez-Heredia, P. Lindberg, A. Martinez Gonzalez, and T. Bolin, "Multipath Simulator Measurements of Handset Dual Antenna Performance with Limited Number of Signal Paths," Antennas and Propagation, IEEE Transactions on, vol. PP, no. 99, pp. 1,0.

[104] 3rd Generation Partnership Project 3GPP, "TS 25.212, Technical Specification Group Radio Access Network; Multiplexing and channel coding (FDD) (Release 10)," Tech. Rep.

[105] L. Greenstein, S. Ghassemzadeh, V. Erceg, and D. G. Michelson, "Theory, experiments, and statistical models," in WPMC'99 Conf, Amsterdam, Sep. 1999.

[106] E. Green, "Radio link design for microcellular systems," BT Tech. J., vol. 8, no. 1, pp. 85-96, 1990.

[107] V. R. Anreddy and M. A. Ingram, "Capacity of measured Ricean and Rayleigh indoor MIMO channels at $2.4 \mathrm{GHz}$ with polarization and spatial diversity," in Proc. IEEE Wireless Communications and Networking Conf. (WCNC'06), vol. 2, Apr. 2006, pp. 946-951.

[108] M. Kang and M. S. Alouini, "Capacity of MIMO Rician channels," IEEE Trans. Wireless Commun., vol. 5, pp. 112-122, Jan. 2006.

[109] R. O. Duda, P. E. Hart, and D. G. Stork, Pattern Classification, 2nd ed. New York: Wiley, 2001.

[110] S. Guatelli et al., "Application of statistical methods for the comparison of data distributions," Nuclear Science Symposium Conference Record, 2004 IEEE, vol. 4, pp. 2086 - 2090, 16-22 Oct. 2004.

[111] L.Råde and B.Westergren, Mathematics handbook for Science and Engineering, 9144031092nd ed.: Studentlitteratur AB, 2003.

[112] P. Hallbjörner, "A Model for the Number of Independent Samples in Reverberation Chambers," Microwave and Optical Technology Letters, vol. 33, no. 1, pp. 25-28, April 2002.

[113] P. Hallbjörner, "Estimating the Number of Independent Samples in Reverberation Chamber Measurements from Sample Differences," IEEE Transactions on Electromagnetic Compatibility, vol. 48, no. 2, pp. 354-358, May 2006.

[114] B. H. Fleury, "First- and second-order characterization of direction dispersion and space selectivity in the radio channel," IEEE Trans. Inf.Theory, vol. 46, no. 6, pp. 2027-2044, Sep. 2000.

[115] C. Tellambura and A. D. S. Jayalath, "Generation of Bivariate Rayleigh and Nakagami-m Fading Envelopes," IEEE Communications Letters, vol. 4, no. 5, pp. 170-172, May 2000.

[116] P. Hallbjörner, J. D. Sánchez-Heredia, E. de los Reyes, and D. A. Sánchez-Hernández, "Limit for the proportion of remaining samples in the mode-stirred chamber sample selection technique," Microwave and Optical Technology Letters, vol. 53, pp. 2608-2610, 2011. 


\section{Appendix A}

\section{A.1 List of Figures}

Figure 1. The coordinate system used in the measurements. SOURCE: [8] ............................................ 12

Figure 2. Proposed two-stage test methodology for MIMO OTA test. SOURCE: [8] ...................................... 13

Figure 3. Example of MIMO/Multiantenna OTA test setup (Uplink signal path omitted in the figure) ........... 14

Figure 4. Mode-stirred chamber setup for devices testing with single cavity. SOURCE: [8] ......................... 16

Figure 5. Mode-stirred chambers with multiple cavities. SOURCE: [8] .............................................. 18

Figure 6. PDP convolution effect observed when a CE and a MSRC are cascaded..................................... 19

Figure 7. DG of a given dual-antenna for calculated at the $99 \%$ signal level reliability (only $1 \%$ of power

levels below the power values used for DG estimation) ....................................................................... 28

Figure 8. Comparison between the scatter plots of a Rayleigh distributed $\mathrm{S}_{21}$ measurement (left) and a

Rician distributed (right). SOURCE: [23] .................................................................................... 30

Figure 9. Data throughput plotted as a function of the received power for a LTE device. SOURCE: [41] ....... 31

Figure 10. Variable $k$-factor in a mode-stirred chamber when altering the azimuth orientation of the

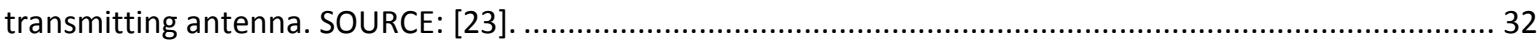

Figure 11. Example of Hyper-Rayleigh fading, compared to Rayleigh and Rician fading. SOURCE: [43]......... 33

Figure 12. Different PDPs (left) and BER (right) measured using an MSRC. SOURCE: [49]. ........................... 35

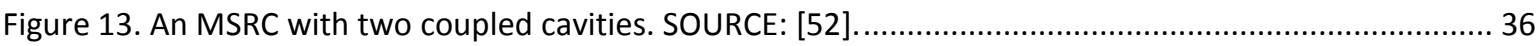

Figure 14. Test bench configuration for test using a MIMO CE cascaded with a MSRC for a $2 \times 2$ MIMO

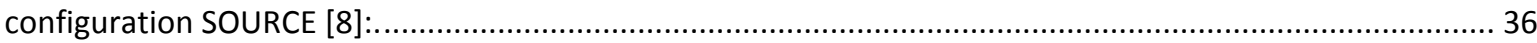

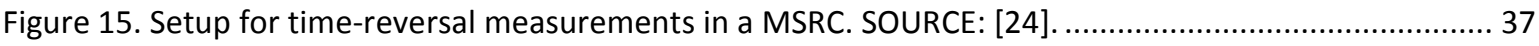

Figure 16. Overview of the different types of cannel models currently used. SOURCE: [57] ...................... 42

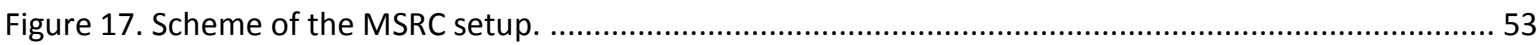

Figure 18. Measurement setup with the presence of the head phantom and the device placed in talk

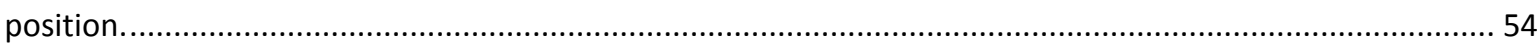

Figure 19. Correlation of the $700 \mathrm{MHz}$ band devices for the three different scenarios. The dotted line corresponds to Prototype A; solid line represents Prototype B......................................................... 55

Figure 20. Correlation of the $2600 \mathrm{MHz}$ band devices for the three different scenarios. The dotted line corresponds to Prototype C; solid line represents Prototype D.......................................................... 56

Figure 21. Power delay profile measured in the MPS for the scenario "B1 LoS". In red the ideal taps of

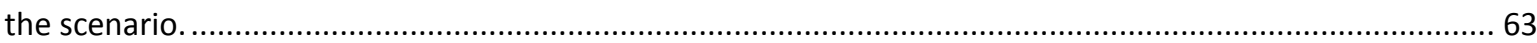

Figure 22. Power delay profile measured in the MPS for the scenario "C2 NLoS". In red the ideal taps of

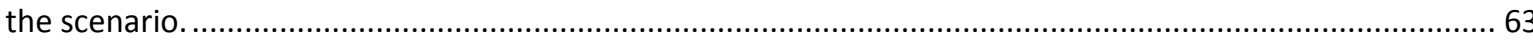

Figure 23. Different phone's orientations used during measurements without user influence....................64 64

Figure 24. Throughput average for all combinations of channel model and phone orientation, without Figure 25. Throughput results for all the positions measured with channel model "B1 LoS". Different

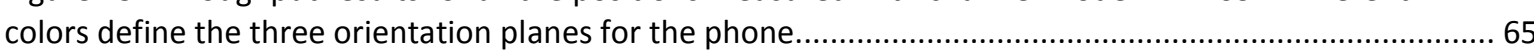
Figure 26. Throughput results for all the positions measured with channel model "C2 NLoS". Different colors define the three orientation planes for the phone.

Figure 27. Standard deviation of the throughput performance for the two channel models under study.

Different colors define the three orientation planes for the phone. ....................................................6. 66

Figure 28. Throughput results for all the user effect cases with the two different channel models.

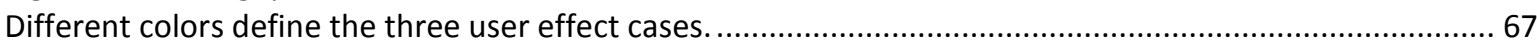

Figure 29. Block diagram of the setup for S-parameter measurements on dual antennas. ............................ 71

Figure 30. Correlation coefficient $\left|\rho_{c}\right|$ of Prototype A (circles) and Prototype B (triangles), as a function of the number of signal paths.

Figure 31. Diversity gain of Prototype A (circles) and Prototype B (triangles), using maximum ratio combining, as a function of the number of signal paths.

Figure 32. Power level where $90 \%$ of maximum throughput is reached, as a function of the number of signal paths. Boxes have lines at the lower quartile, median (bold line), and upper quartile values. Whiskers show the extent of the rest of the data. 
Figure 33. Power level where $10 \%$ of maximum throughput is reached, as a function of the number of signal paths. Boxes have lines at the lower quartile, median (bold line), and upper quartile values.

Whiskers show the extent of the rest of the data.

Figure 34. HSDPA throughput standard deviation as a function of received power.....................................79

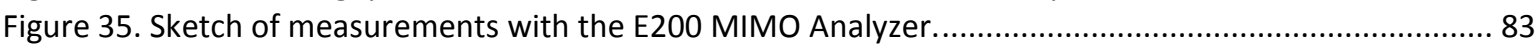

Figure 36. Scatter plots for mode-stirred and mode-processed data. .......................................................... 84

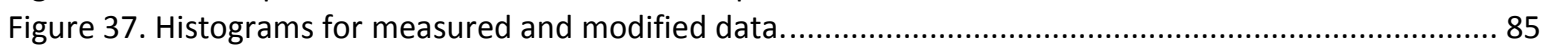

Figure 38. Measured and emulated capacity vs. SNR for 3x3 MIMO system A. ....................................... 86

Figure 39. Emulated correlation coefficients between adjacent antennas for the MIMO systems in Table 20.

Figure 40. Cumulative probability density function vs. relative power for system B with different $k$ -

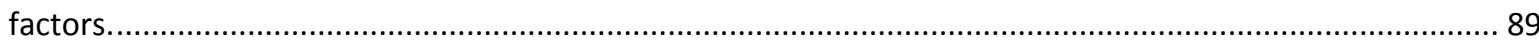

Figure 41. DG versus $k$-factor with probability level as parameter, for all tested systems..........................90

Figure 42. Measured capacity of all tested systems vs. SNR for different $k$-factors. .....................................91

Figure 43. Measured capacity of all tested systems vs. $k$-factor for different SNR values............................92

Figure 44. A two-cavity MSRC. ........................................................................................................ 100

Figure 45. Histogram of measured AoA using the high resolution MUSIC algorithm for the initial Rayleigh

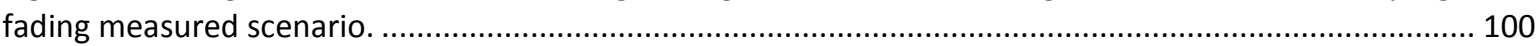

Figure 46. Initial (ideal theoretical Rayleigh) and target and target (ideal theoretical Rician) fading distributions.

Figure 47. A comparison between initial measured Rayleigh-fading set, processed sample subset, and Rician-fading target 4.

Figure 48. The amount of remaining samples for each of the algorithms vs. the $k$-factor. The target distribution of Rice $1(k=0.5)$, Rice $2(k=2)$ and Rice $3(k=8)$ with the same mean output power as the original distribution are indicated by points. The lines are added as guide for the eye.

Figure 49. Distribution error of the three algorithms vs. the $k$-factor. The target distribution of Rice 1

$(k=0.5)$, Rice $2(k=2)$ and Rice $3(k=8)$ are indicated by points.

Figure 50. The ratio $P_{\text {out }} / P_{\text {in }}$ vs. amount of remaining samples for each algorithm. All three distributions have the same $k$-factor, $k=2$.

Figure 51. Correlation as a function of Rician $k$-factor of a high correlated prototype (Prototypes C and D defined in Chapter 5)

Figure 52. DG as a function of Rician $k$-factor of a high correlated prototype (Prototype $C$ defined in Chapter 5).

Figure 53. 3×3 MIMO capacity emulated using the simple selection technique with different Rician $k$ factors

Figure 54. PDFs for $\rho_{i}=0$ and $\rho_{t}=0.3,0.5,0.7$, and 0.9. $N_{i}=10000$ in all cases. Numbers next to the peaks are $N_{t}$ values. Histograms are the results of simulations with 500 realizations. Solid lines are spreads due to finite sequence length for the $N_{t}$ values at hand.

Figure 55. PDFs for $\rho_{i}=0.7$ and $\rho_{t}=0.3,0.5,0.7$, and $0.9 . N_{i}=10000$ in all cases. Numbers next to the peaks are $N_{t}$ values. Histograms are the results of simulations with 500 realizations. Solid lines are spreads due to finite sequence length for the $N_{t}$ values at hand.

Figure 56. Diversity gain after sample selection, for the different values of target correlation coefficient. . 112 Figure 57. Number of remaining samples after sample selection, as a percentage of the number of initial samples $\left(\rho_{i}=0.05\right)$. 


\section{A.2 List of Tables}

Table 1. Expanded Uncertainty Maximum Limits (in dB) for Different Configurations for TRP and TIS (SOURCE: [5]).

Table 2. TRP minimum performance requirement for FDD roaming bands in the speech position and the primary mechanical mode. (SOURCE: [6])

Table 3. TRS minimum requirements for FDD roaming bands in the speech position for the primary mechanical mode. (SOURCE: [6])

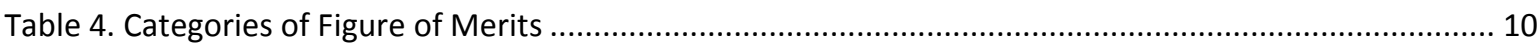

Table 5. Characteristic comparison between the three proposed OTA methodologies. SOURCE: [8]............ 20

Table 6. Most relevent parameters in the SCME cannel models. SOURCE: [11] ..................................... 44

Table 7. A1 LoS simplified WINNER II cannel model parameters. SOURCE: [13] ......................................47

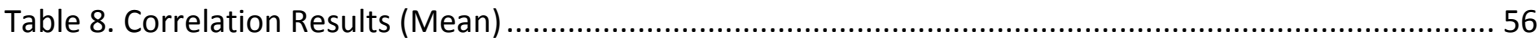

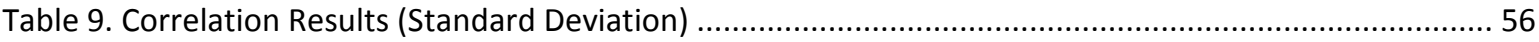

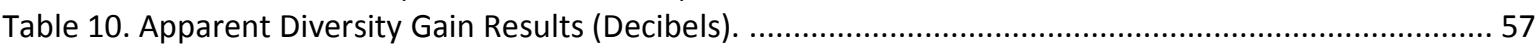

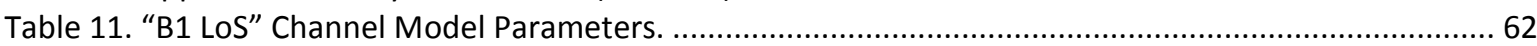

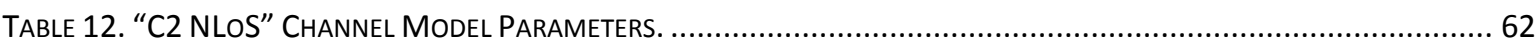

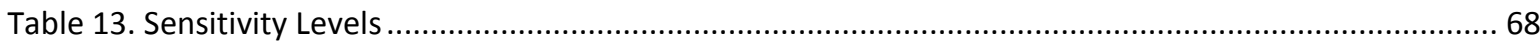

Table 14. Phase sweep rate on the MPS branches, and the active branches used for the different number of paths. For 2,4 , and 8 paths, three sets are used, each given by a column......................................... 72

Table 15. Proportion of correlation coefficient values less than 0.7 for Prototypes $A$ and $B$, for different

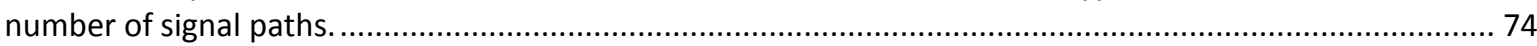

Table 16. Best case, median, and worst case Apparent diversity gain of Prototypes A and B, for different

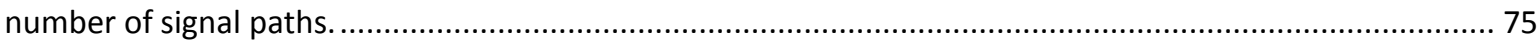

Table 17. Best case, median, mean, and worst case at $90 \%$ throughput level, for different number of signal paths

Table 18. Best case, median, mean, and worst case at $10 \%$ throughput level, for different number of

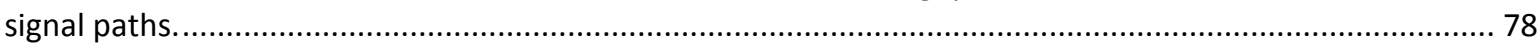

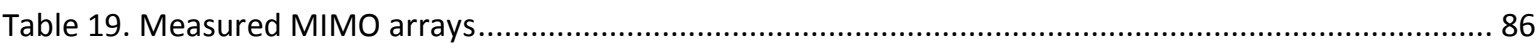

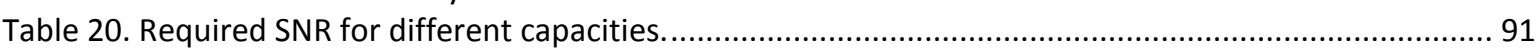

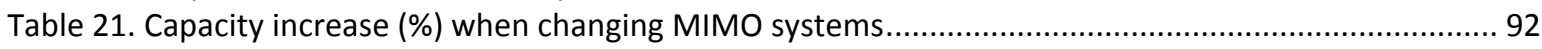

Table 22. The five different target Rice distributions .............................................................................. 101

Table 23. Performance of Sample-Selection Technique With GA ..................................................... 102 